\title{
Microstructure and Performance Studies of a Novel Cobalt High Entropy Alloy
}

\author{
by \\ Xueyao Wu
}

A thesis submitted to the Faculty of Graduate and Postdoctoral Affairs in partial fulfillment of the requirements for the degree of

\author{
Master of Applied Science \\ in \\ Mechanical Engineering
}

Carleton University

Ottawa, Ontario

(C)2020, Xueyao Wu 


\section{Abstract}

A novel high entropy alloy (HEA), designated as HE6, is created by combining the features of HEAs and Stellite alloys in this research. The new alloy has the equiatomic Co-Cr-Fe-Ni composition (22 at.\%) with a large amount of W (19 wt.\%), small amounts of C (0.96 wt.\%) and Mo (3 wt.\%). The bulk specimens of HE6 alloy are fabricated from the alloy powder via spark plasma sintering (SPS) and plasma transferred arc (PTA) welding processes. The microstructures of the SPS and PTA specimens are studied using a scanning electron microscope (SEM) equipped with energy dispersive X-ray spectroscopy (EDX) and using X-ray diffraction (XRD). A series of material characterization tests such as hardness, wear and corrosion are performed on the bulk HE6 specimens. As the benchmark of Stellite alloy family, Stellite 6 is investigated along with HE6 for comparison. It is found that HE6 alloy has a microstructure which is similar to that of Stellite alloys where various carbides and intermetallics are embedded in a solid solution matrix, but compared to Stellite 6, the FCC solid solution of HE6 consists of multi-element structures (Co, $\mathrm{Cr}, \mathrm{Fe}$ and $\mathrm{Ni}$ ), not single FCC Co structure, and also, the carbides and intermetallics in HE6 alloy are more diverse. The hardness and dry-sliding wear tests show that HE6 alloy does not perform as well as Stellite 6 . In the electrochemical and immersion corrosion tests, similar to Stellite alloys, HE6 alloy displays passivation ability by forming protective $\mathrm{Cr}$-rich oxide films in $3.5 \% \mathrm{NaCl}, 2 \%$ $\mathrm{HCl}$ and $10 \% \mathrm{H}_{2} \mathrm{SO}_{4}$ solutions, but localized corrosion (pitting) can occur when the oxide films are broken. HE6 alloy shows less resistance to corrosion under the electrochemical impedance spectroscopy (EIS) and cyclic polarization tests than Stellite 6, but has lower corrosion rates under immersion test in $5 \% \mathrm{HCl}$ and $10 \% \mathrm{H}_{2} \mathrm{SO}_{4}$ solutions for the longer testing duration (72 hours), also showing nearly stable corrosion rate with testing time, which indicates better repairing ability of the oxide films. 


\section{Acknowledgements}

It is of great honor for me to appreciate many people who enable me to finish the tasks and research.

I would like to first thank my supervisor, Professor Rong Liu, for allowing me to conduct this interesting research. Her great patience and professional guidance have inspired me in academia and will continue to influence me for many years to come.

My sincere thanks to Dr. Matthew Yao from Kennametal Stellite Inc. for mentoring me during the entire journey. I cannot achieve this stage without his supports.

I would like to express my thanks to Dr. Xiaozhou (Joe) Zhang for his selfless and constant supports from all aspects of daily life. I really appreciate working with him.

Also, my work wouldn't be accomplished without the help of Dr. Jianqun (JJ) Wang, for his great guidance on SEM/EDX operations. He is always available when I need help. And I want to thank Dr. Jeffrey Ovens from uOttawa for his support of conducting the XRD experiment in a very timely manner. They saved my life.

Moreover, I must thank my colleagues and friends, Yinping Ding, Yinda Cheng, Siqi Li, Leigh Corrigall, and Peng $\mathrm{Xu}$ for their generous supports and encouragement all along.

Last but not the least, I want to send my gratefulness to my parents, grandparents and family. In the best way, they make my dreams come true. 


\section{Table of Contents}

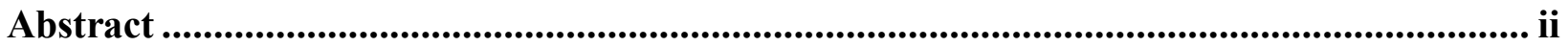

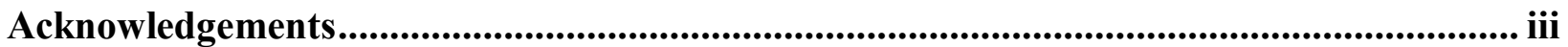

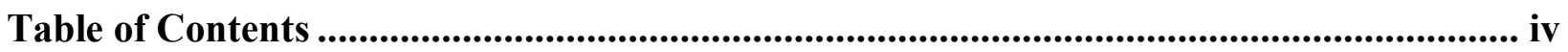

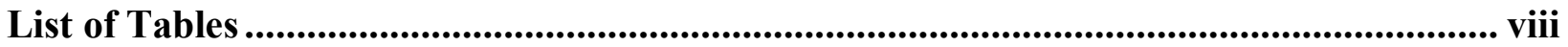

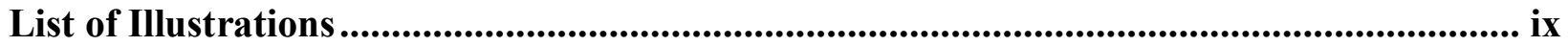

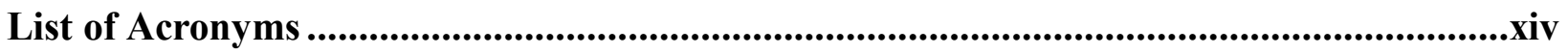

Chapter 1: Introduction ............................................................................................................

$1.1 \quad$ Research Background and Significance ...........................................................

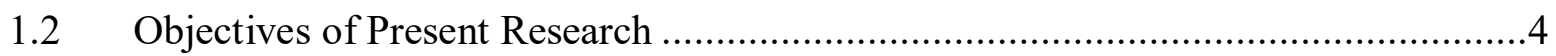

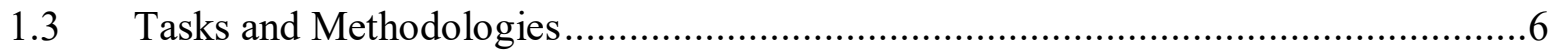

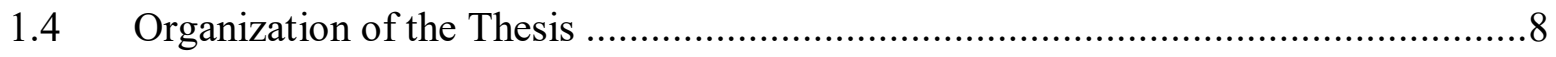

Chapter 2: Literature Review .......................................................................................................10

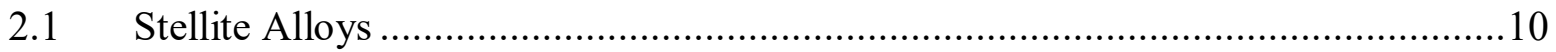

2.1.1 Alloying elements and precipitates of Stellite alloys .......................................12

2.1.2 Previous research on Stellite 6 alloy ...........................................................15

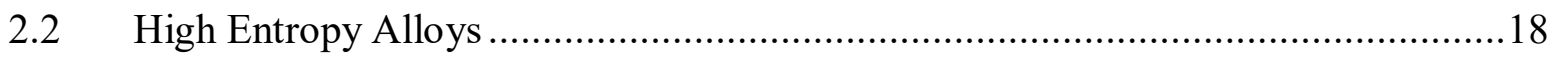

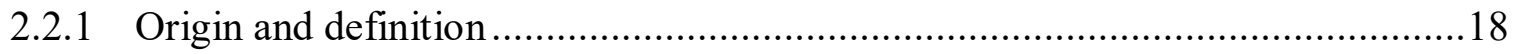

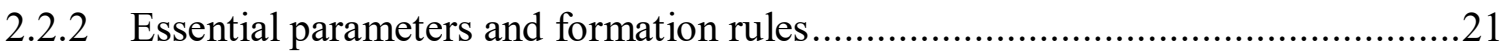

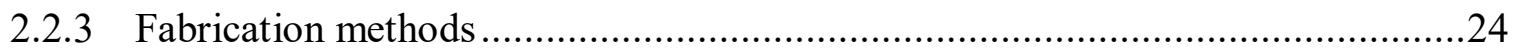

2.2.3.1 Plasma transferred arc (PTA) welding .................................................25

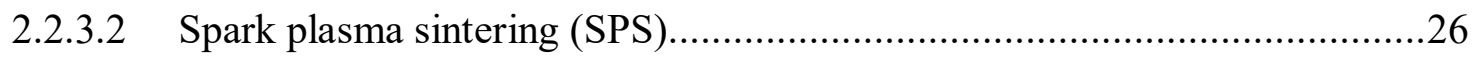

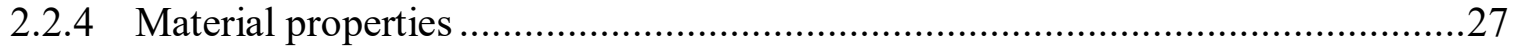

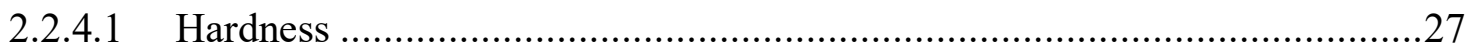


2.2.4.2 Yield strength and ductility...........................................................28

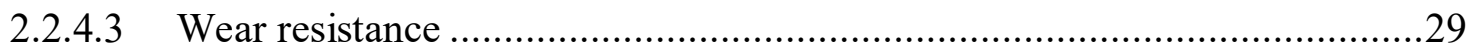

2.2.4.4 Corrosion resistance ....................................................................... 30

2.3 Material Performance Testing Methods ................................................. 32

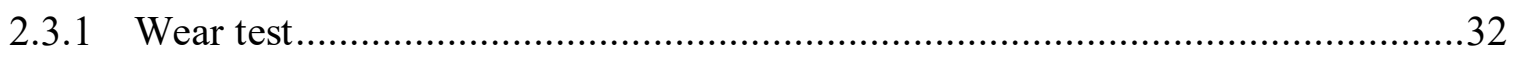

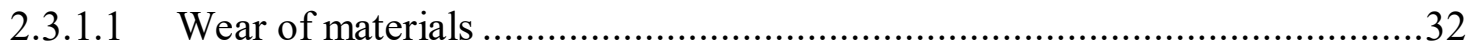

2.3.1.2 Pin-on-disk wear test .................................................................. 32

2.3.1.3 Related researches in wear test ........................................................35

2.3.2 Corrosion test.................................................................................. 37

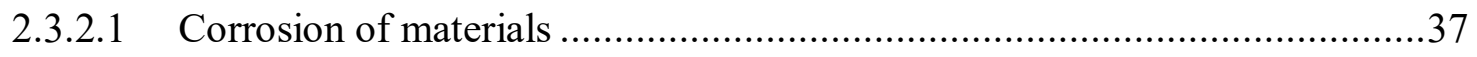

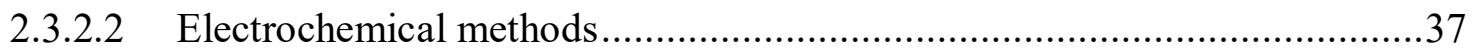

2.3.2.3 Immersion method...................................................................... 42

2.3.2.4 Related studies in corrosion test..................................................42

Chapter 3: Alloy Powder Characterization and Bulk Alloy Microstructural Analysis...45

3.1 Raw Powder Specification and Examination ................................................45

3.1.1 Chemical composition..................................................................... 45

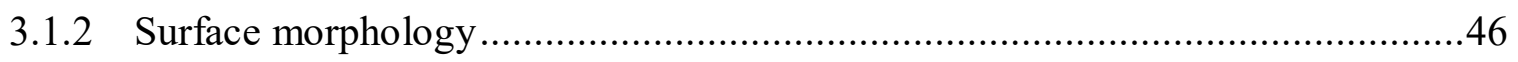

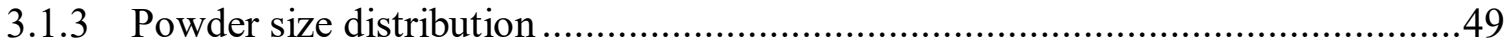

3.1.4 Differential scanning calorimetry (DSC) analysis .....................................52

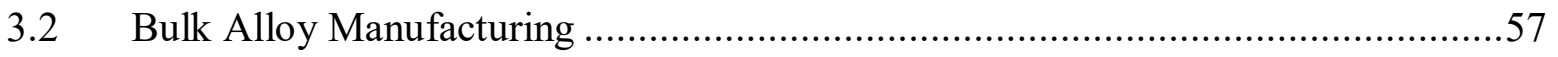

3.2.1 Plasma transferred arc (PTA) welding process ........................................57

3.2.2 Spark plasma sintering (SPS) process ...............................................5

3.2.3 Density measurement of bulk alloy specimens .......................................61 


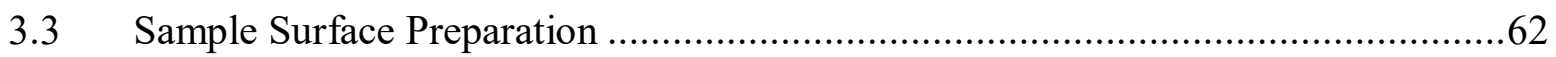

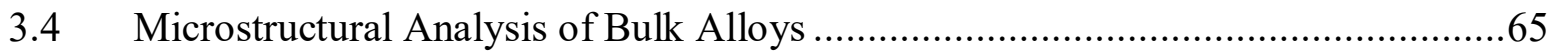

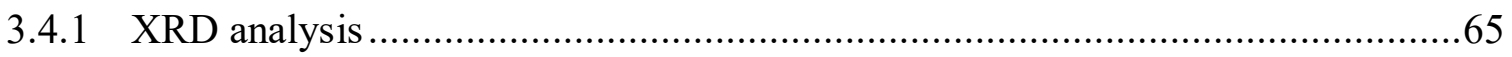

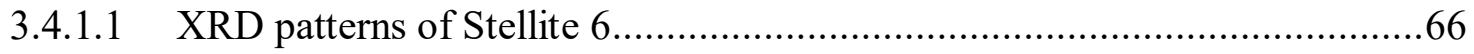

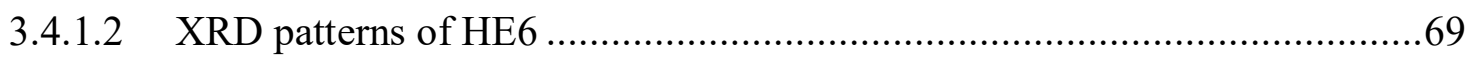

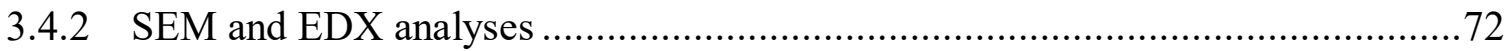

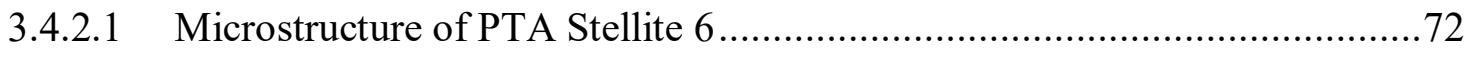

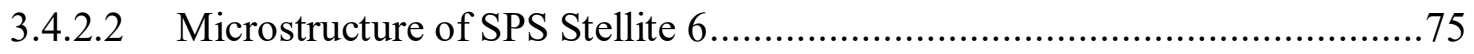

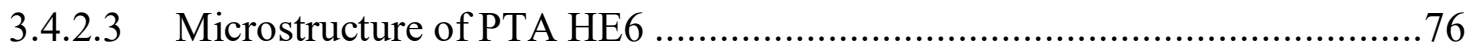

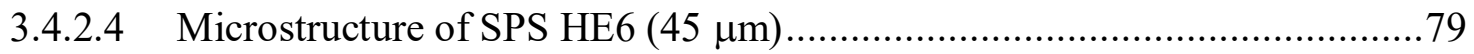

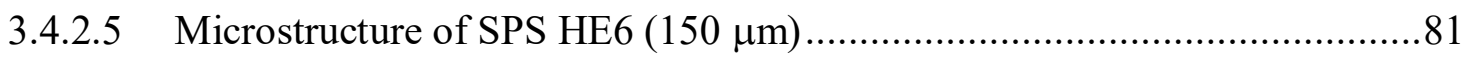

3.4.3 Determination of carbide volume fraction .....................................................83

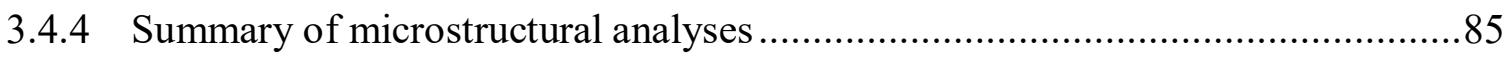

Chapter 4: Performance Evaluation of Bulk Alloys ...............................................................90

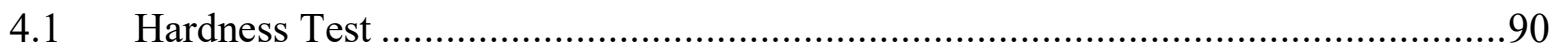

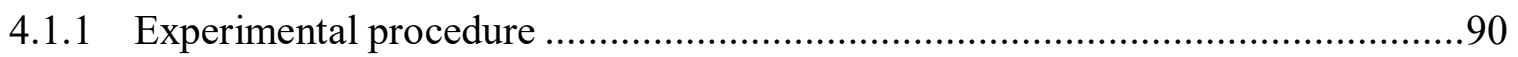

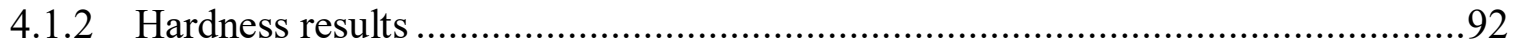

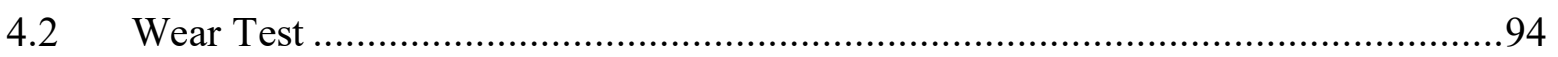

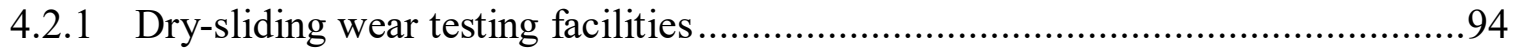

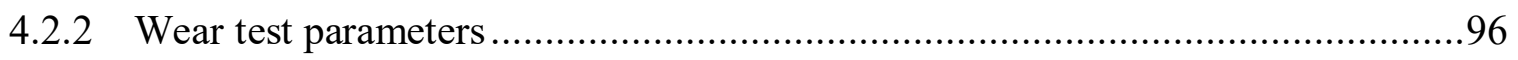

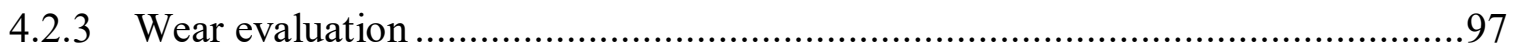

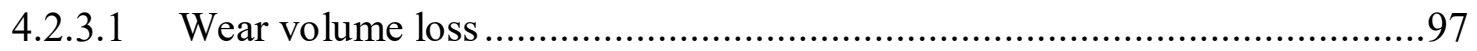

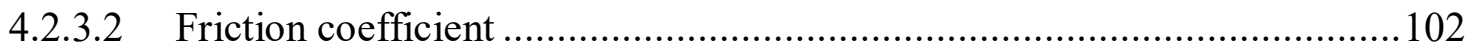




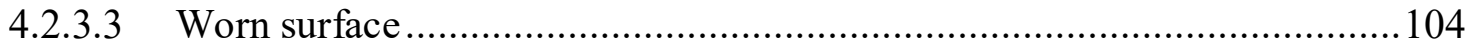

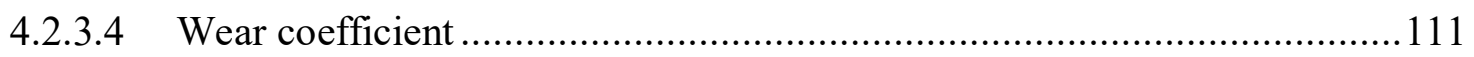

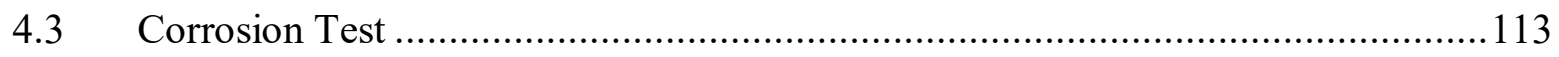

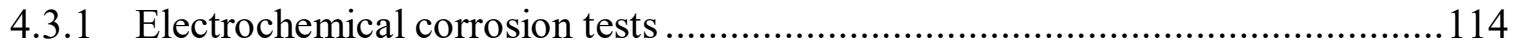

4.3.1.1 Specimen and solution preparation ....................................................114

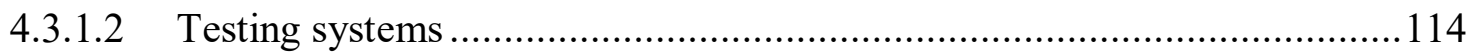

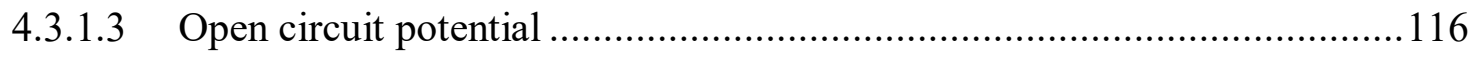

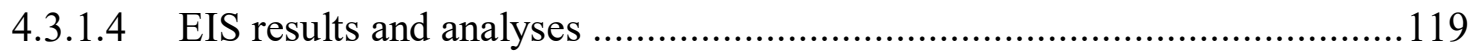

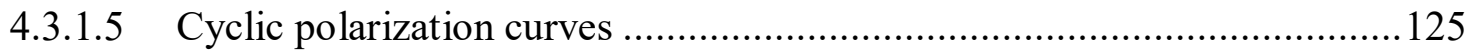

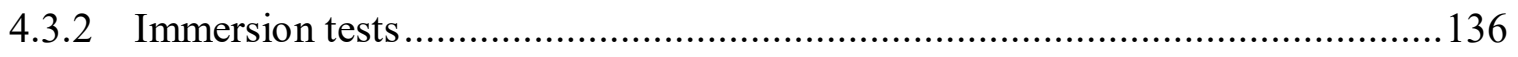

4.3.2.1 Specimen and solution preparation ........................................................ 136

4.3.2.2 Experiment setup and procedure …………..........................................137

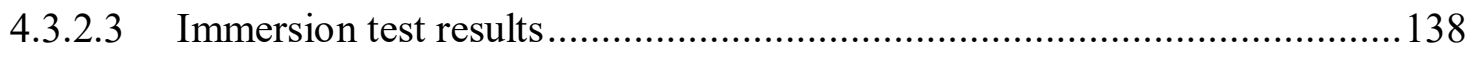

4.3.2.4 Morphology analysis of immersion-tested specimens .............................. 140

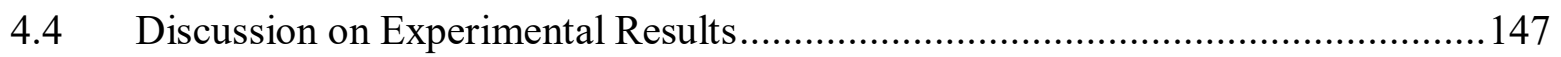

Chapter 5: Conclusions and Future Work .............................................................................. 149

$5.1 \quad$ Summary of the Work Completed ...................................................................... 149

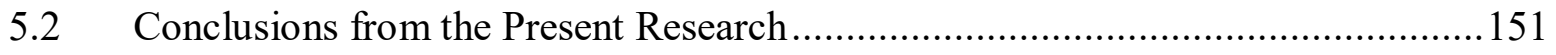

5.3 Significant Contributions of the Present Research .............................................. 153

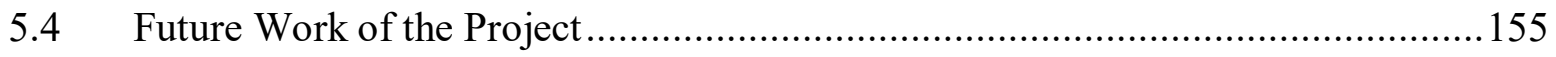

Bibliography .................................................................................................................................156 


\section{List of Tables}

Table 2-1: Chemical compositions (wt.\%) of Stellite alloys [2].

Table 2-2: Entropy values calculated for typical traditional alloys at their liquid state or random state [38]. .20

Table 2-3: Entropy values calculated for typical Stellite alloys. ..............................................

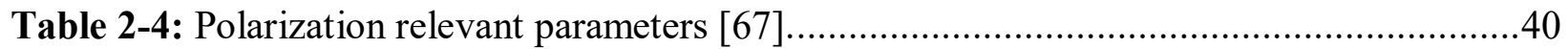

Table 2-5: Chemical compositions (wt.\%, Co in balance) of Stellite alloys [76]. ......................43

Table 2-6: Summary of polarization test results of Stellite alloys in $3.5 \mathrm{wt} . \% \mathrm{NaCl}$ solution [76].

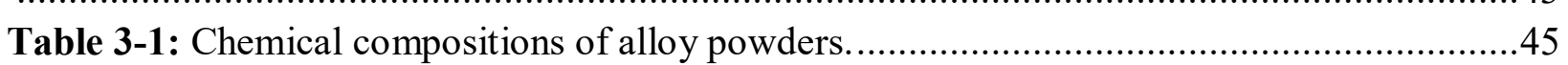

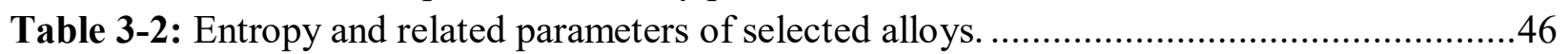

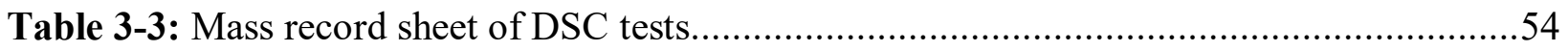

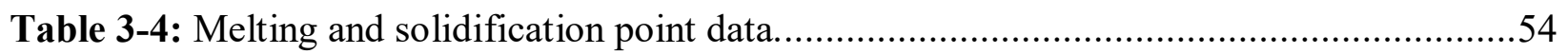

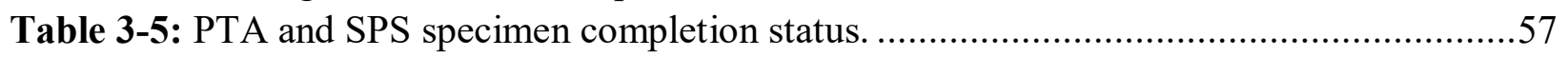

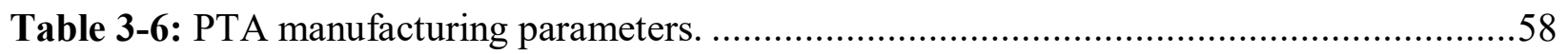

Table 3-7: SPS process parameters for alloy specimen manufacturing. .....................................60

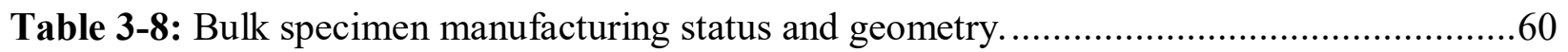

Table 3-9: Density and porosity measurements of alloy specimens. ........................................61

Table 3-10: A summary of the surface requirements for various experiments. …………...........62

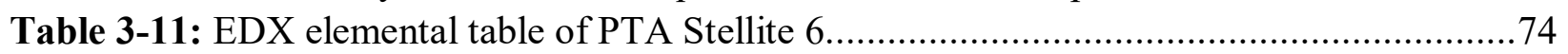

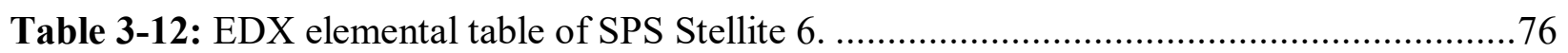

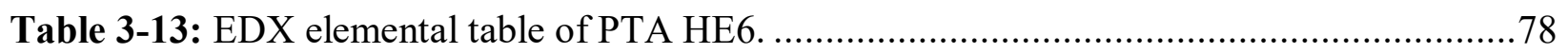

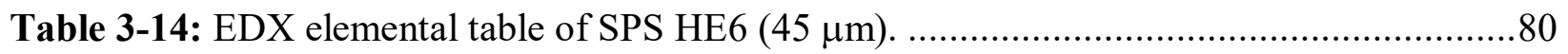

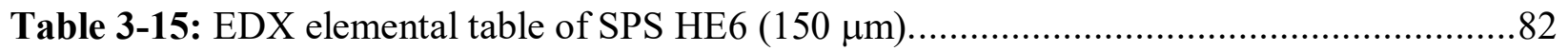

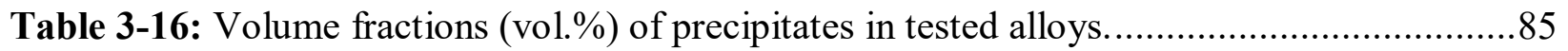

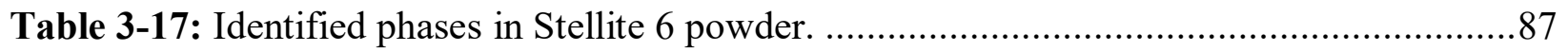

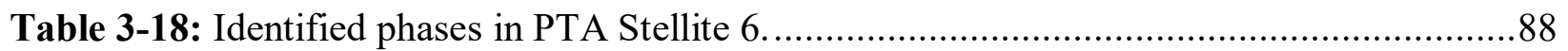

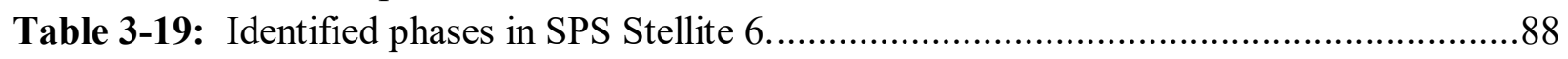

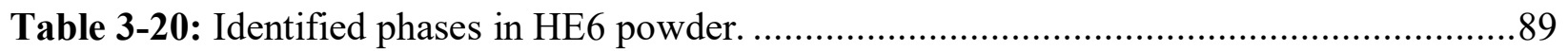

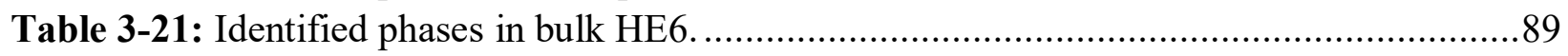

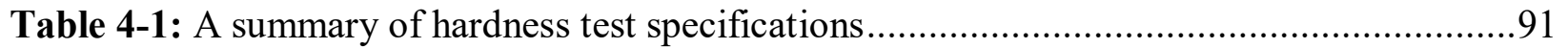

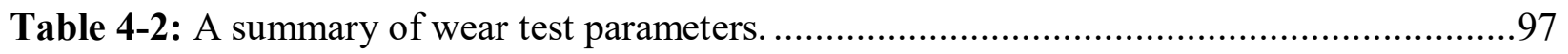

Table 4-3: EIS results of the tests in $3.5 \% \mathrm{NaCl}$ solution fitted from the equivalent circuit. ....121

Table 4-4: EIS results of the tests in $2 \% \mathrm{HCl}$ solution fitted from the equivalent circuit.......... 123

Table 4-5: EIS results of the tests in $10 \% \mathrm{H}_{2} \mathrm{SO}_{4}$ solution fitted from the equivalent circuit.... 124

Table 4-6: Summary of polarization test results of the alloys in 3.5\% NaCl solution...............129

Table 4-7: Summary of polarization test results of the alloys in $\% \mathrm{HCl}$ solution. ...................132

Table 4-8: Summary of polarization test results of the alloys in $10 \% \mathrm{H}_{2} \mathrm{SO}_{4}$ solution...............135

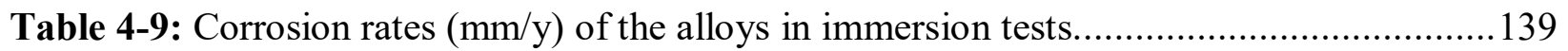

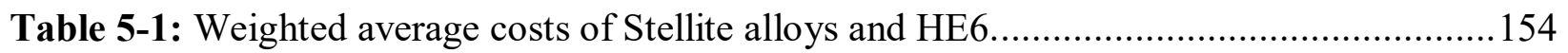




\section{List of Illustrations}

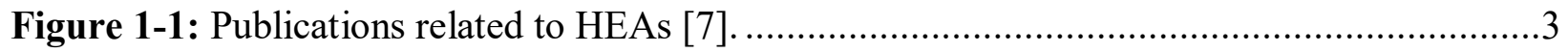
Figure 2-1: Typical microstructure of laser cladding of (a) Stellite 6, (b) Stellite 12, (c) Stellite 1 [28].

Figure 2-2: Total weight loss measurements of Stellite alloys and stainless steels in $3.5 \% \mathrm{NaCl}$

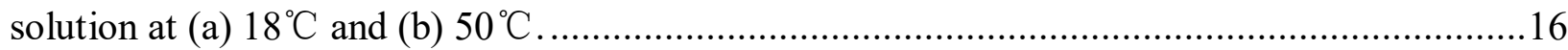

Figure 2-3: Low-stress abrasive and high-stress abrasive-wear resistance of Stellite alloys.......17 Figure 2-4: Corrosion resistance of W-containing Stellite alloys and Mo-containing Stellite

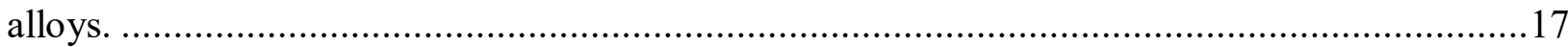

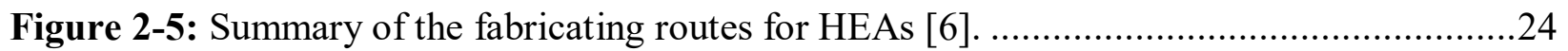

Figure 2-6: Schematic diagram of plasma transferred arc (PTA) process. ................................25

Figure 2-7: Schematic illustration of the working principle of an SPS apparatus.......................26

Figure 2-8: Hardness values of most studied HEAs, compared with conventional alloys. .........28

Figure 2-9: Tensile yield stress versus ductility of most studied HEAs at room temperature,

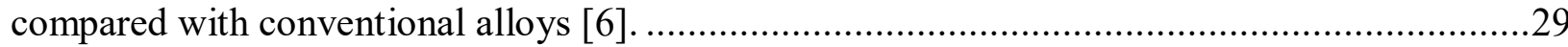

Figure 2-10: Pin-on-disk adhesive wear resistance versus hardness of HEAs and Steels [54]....30 Figure 2-11: A comparison to average corrosion rates ( $\mathrm{mm} /$ year) between HEAs and other materials in $3.5 \mathrm{wt} . \% \mathrm{NaCl}$ solution at room temperature. ................................................... 31

Figure 2-12: A comparison in average corrosion rates ( $\mathrm{mm} /$ year) between HEAs and ................31

Figure 2-13: Mechanism of adhesive wear of materials [60]...................................................3

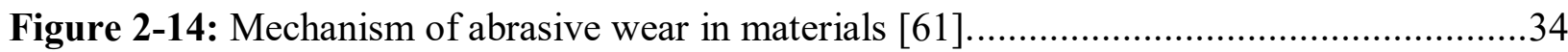

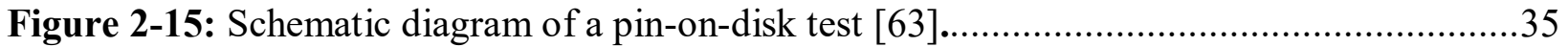

Figure 2-16: Wear volume losses of different alloys under high-temperature ball-on-disc sliding

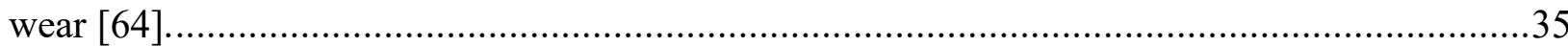

Figure 2-17: Comparison of wear rate between Stellite alloy hardfacings [65]........................36

Figure 2-18: A typical hypothetical polarization curve with relevant parameters [73]..............39

Figure 2-19: A typical diagram of electrochemical impedance spectroscopy (EIS) [29]...........41

Figure 2-20: Potentiodynamic polarization curves of Stellite alloys in $3.5 \mathrm{wt} . \% \mathrm{NaCl}$ solution

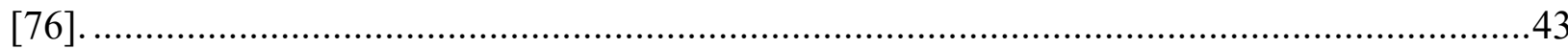

Figure 3-1: Tescan scanning electron microscope (SEM) system......................................47 Figure 3-2: SEM microstructure of Stellite 6 powder with $45 \mu \mathrm{m}$ (a) low magnification, (b) high

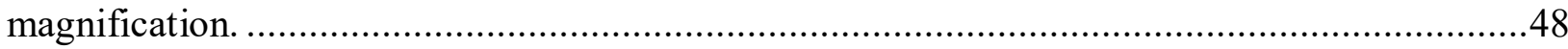

Figure 3-3: SEM microstructure of HE6 powder with $45 \mu \mathrm{m}$ (a) low magnification, (b) high magnification. 48

Figure 3-4: SEM microstructure of HE6 powder with $150 \mu \mathrm{m}$ (a) low magnification, (b) high magnification.

Figure 3-5: An ImageJ processing result of powder size distribution (a) original SEM image of

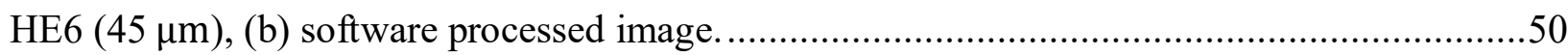

Figure 3-6: Particle size distribution of Stellite $6(45 \mu \mathrm{m})$ powder...........................................51 
Figure 3-7: Particle size distribution of HE6 $(45 \mu \mathrm{m})$ powder...........................................51

Figure 3-8: Particle size distribution of HE6 $(150 \mu \mathrm{m})$ powder.............................................52

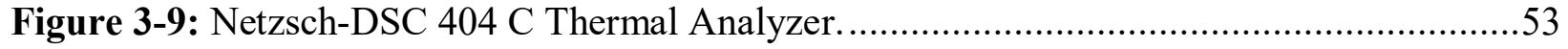

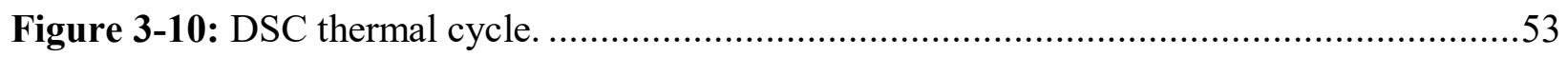

Figure 3-11: DSC curves of Stellite $6(45 \mu \mathrm{m})$ powder................................................55

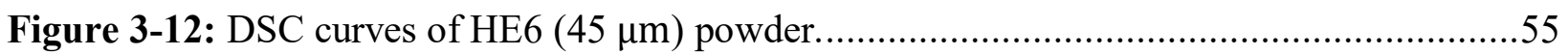

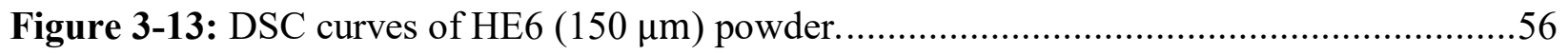

Figure 3-14: DSC heating curves of tested powders.....................................................56

Figure 3-15: DSC cooling curves of tested powders. .......................................................57

Figure 3-16: An example of wire cutting on a PTA workpiece. .......................................58

Figure 3-17: Schematic of the working principle of the SPS apparatus [45].........................59

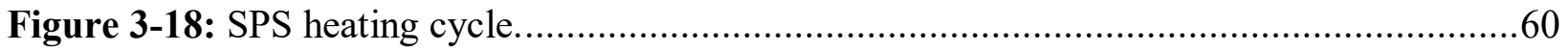

Figure 3-19: Buehler Simplimet II Mounting Press. .................................................63

Figure 3-20: Buehler MetaServ ${ }^{\mathrm{TM}} 250$ Grinder-Polisher................................................63

Figure 3-21: CO-Z 6L Professional Ultrasonic Cleaner. .........................................................64

Figure 3-22: Combined XRD patterns of Stellite 6 alloy in powder and bulk form. ................66

Figure 3-23: XRD pattern and phase identification of Stellite 6 powder. ...............................67

Figure 3-24: XRD pattern and phase identification of PTA Stellite 6 .................................68

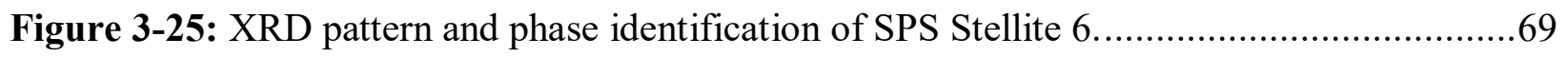

Figure 3-26: Combined XRD patterns of HE6 powders with different particle sizes. ...............70

Figure 3-27: Combined XRD patterns of HE6 bulk specimens from SPS and PTA processes...70

Figure 3-28: XRD pattern and phase identification of HE6 powder $(150 \mu \mathrm{m}) \ldots \ldots \ldots \ldots \ldots \ldots \ldots . . . . . . . . . . .11$

Figure 3-29: XRD pattern and phase identification of SPS HE6 $(150 \mu \mathrm{m})$..........................72

Figure 3-30: PTA Stellite 6 microstructure (a) at low magnification, (b) at high magnification. 73

Figure 3-31: EDX spectra of PTA Stellite 6 microstructure: (a) locations of spectrum, (b) spectrum 1 of grey area (Co-based solid solution), spectrum 2 of dark area (Cr-rich carbide), spectrum 3 of bright area (W/Mo-rich intermetallic)..................................................... 74

Figure 3-32: SPS Stellite 6 microstructure (a) at low magnification, (b) at high magnification.. 75 Figure 3-33: EDX spectra of SPS Stellite 6 microstructure: (a) locations of spectrum, (b) spectrum 1 of grey area (Co-based solid solution), spectrum 2 of dark grey area (Cr-rich

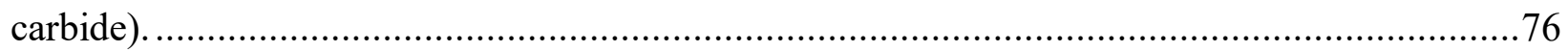

Figure 3-34: PTA HE6 microstructure (a) at low magnification, (b) at high magnification. .....77 Figure 3-35: EDX spectra of PTA HE6 microstructure: (a) locations of spectrum, (b) spectrum 1 of grey area (solid solution), spectrum 2 of bright area (W/Mo-rich intermetallic), spectrum 3 of

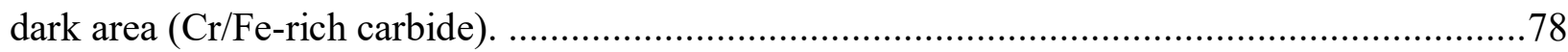

Figure 3-36: SPS HE6 (45 $\mu \mathrm{m})$ microstructure (a) at low magnification, (b) at high magnification.

Figure 3-37: EDX spectra of SPS HE6 $(45 \mu \mathrm{m})$ microstructure: (a) locations of spectrum, (b) spectrum 1 of grey area (solid solution), spectrum 2 of bright area (W/Mo-rich phase), spectrum 3 of dark area (similar to grey area). 
Figure 3-38: SPS HE6 $(150 \mu \mathrm{m})$ microstructure (a) at low magnification, (b) at high magnification.

Figure 3-39: EDX spectra of SPS HE6 $(150 \mu \mathrm{m})$ microstructure: (a) locations of spectrum, (b) spectrum 1 of grey area (solid solution), spectrum 2 of bright area (W/Mo-rich phase), spectrum 3 of dark area (carbide). .82

Figure 3-40: ImageJ processing of PTA Stellite 6 (a) original SEM image, (b) Cr-rich carbides highlighted, (c) W/Mo-rich intermetallics highlighted. .83

Figure 3-41: ImageJ processing of SPS Stellite 6 (a) original SEM image, (b) $\mathrm{M}_{23} \mathrm{C}_{6}$ carbides highlighted. (c) $\mathrm{M}_{2} \mathrm{C}$ carbides highlighted.

Figure 3-42: ImageJ processing of PTA HE6 (a) original SEM image, (b) $\mathrm{Cr}$, $/ \mathrm{Fe}$-rich carbides highlighted, (c) W/Mo-rich intermetallics/carbides highlighted.

Figure 3-43: ImageJ processing of SPS HE6 $(45 \mu \mathrm{m})$ (a) original SEM image, (b) W/Mo-rich intermetallics/carbides highlighted. .84

Figure 3-44: ImageJ processing of SPS HE6 $(150 \mu \mathrm{m})$ (a) original SEM image, (b) W/Mo-rich

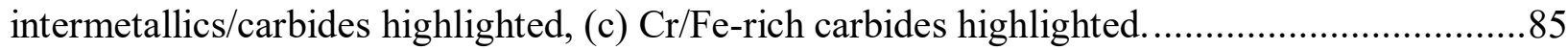

Figure 4-1: CLEMEX Microhardness tester, model SMT-X7 Dual Indenter............................91

Figure 4-2: Diagonal lengths of an indentation. ................................................................... 91

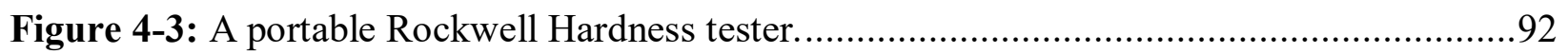

Figure 4-4: Vickers Hardness test results of bulk alloy specimens. .......................................93

Figure 4-5: Rockwell Hardness test results of bulk alloy specimens. .....................................93

Figure 4-6: Pin-on-disc tribometer: (a) system assembly, (b) detailed specimen positioning. ....94

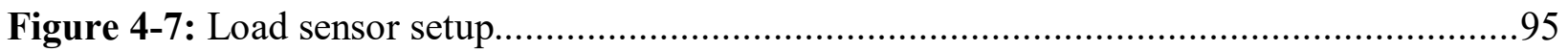

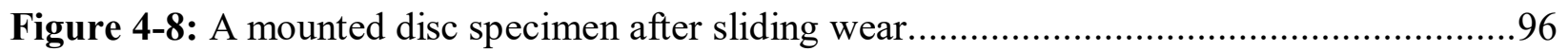

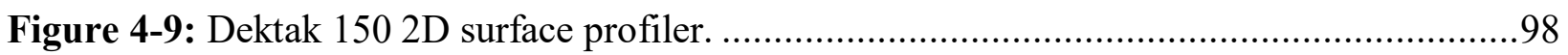

Figure 4-10: An example of cross-sectional profile of wear track from the 2D profiler.............98

Figure 4-11: Illustration of four measuring locations on a wear track...................................99

Figure 4-12: Wear track of (a) PTA Stellite 6, (b) PTA HE6, (c) SPS Stellite 6, (d) SPS HE6 (45 $\mu \mathrm{m})$, (e) SPS HE6 $(150 \mu \mathrm{m})$.

Figure 4-13: Comparison of the cross-sectional profiles of wear tracks between tested alloys. 101 Figure 4-14: Wear volume losses of tested alloys at $50 \mathrm{rpm}$ under $15 \mathrm{~N}$ for 2 hour dry sliding.

Figure 4-15: Friction coefficient versus sliding distance. 103

Figure 4-16: Average friction coefficients of tested alloys. 104

Figure 4-17: SE image of worn surface of PTA Stellite 6 at (a) low magnification, (b) medium magnification, (c) high magnification. 105

Figure 4-18: SE image of worn surface of SPS Stellite 6 at (a) low magnification, (b) medium magnification, (c) high magnification. 106

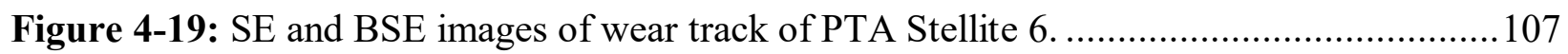

Figure 4-20: SE and BSE images of wear track of SPS Stellite 6. 107 
Figure 4-21: SE image of worn surface of PTA HE6 at (a) low magnification, (b) medium magnification, (c) high magnification.

Figure 4-22: SE image of worn surface of SPS HE6 (45 $\mu \mathrm{m})$ at (a) low magnification, (b) medium magnification, (c) high magnification.

Figure 4-23: SE image of worn surface of SPS HE6 $(150 \mu \mathrm{m})$ at (a) low magnification, (b) medium magnification, (c) high magnification.

Figure 4-24: SE and BSE images of wear track of PTA HE6.

Figure 4-25: SE and BSE images of wear track of SPS HE6 $(45 \mu \mathrm{m})$.

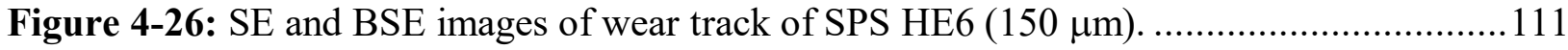

Figure 4-27: Wear coefficients of tested alloys.

Figure 4-28: Electrochemical corrosion system setup: (a) control system, (b) K0235 flat corrosion cell.

Figure 4-29: Schematic diagram of the electrochemical cell in a $3.5 \mathrm{wt} . \% \mathrm{NaCl}$ corrosion test [97].

Figure 4-30: OCP curves of alloy specimens in (a) $3.5 \% \mathrm{NaCl}$, (b) $2 \% \mathrm{HCl}$, (c) $10 \% \mathrm{H}_{2} \mathrm{SO}_{4}$

solution.

Figure 4-31: Simplified Randles circuit.

Figure 4-32: Nyquist plot of PTA Stellite 6 tested in $3.5 \% \mathrm{NaCl}$ solution............................ 120

Figure 4-33: Nyquist plot of PTA HE6 tested in 3.5\% $\mathrm{NaCl}$ solution................................... 121

Figure 4-34: Nyquist plot of PTA Stellite 6 tested in 2\% $\mathrm{HCl}$ solution. ................................. 122

Figure 4-35: Nyquist plot of PTA HE6 tested in 2\% $\mathrm{HCl}$ solution. ...................................... 122

Figure 4-36: Nyquist plot of PTA Stellite 6 tested in $10 \% \mathrm{H}_{2} \mathrm{SO}_{4}$ solution........................... 123

Figure 4-37: Nyquist plot of PTA HE6 tested in $10 \% \mathrm{H}_{2} \mathrm{SO}_{4}$ solution. ................................. 124

Figure 4-38: Comparison of $R p$ value from EIS tests............................................ 125

Figure 4-39: Corrosion test specimen showing the exposed area to the electrolyte................ 126

Figure 4-40: Cyclic polarization curves of PTA Stellite 6 alloy tested in 3.5\% $\mathrm{NaCl}$............ 127

Figure 4-41: Cyclic polarization curves of PTA HE6 alloy tested in 3.5\% NaCl. ................. 127

Figure 4-42: Comparison of polarization behavior between PTA Stellite 6 and PTA HE6 in $3.5 \% \mathrm{NaCl}$ solution. ........................................................................................... 129

Figure 4-43: Cyclic polarization curves of PTA Stellite 6 alloy tested in 2\% HCl solution...... 130

Figure 4-44: Cyclic polarization curves of PTA HE6 alloy tested in 2\% $\mathrm{HCl}$ solution. ........... 130

Figure 4-45: Comparison of cyclic polarization behavior between PTA Stellite 6 and PTA HE6

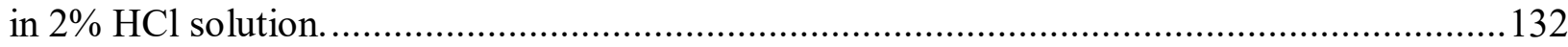

Figure 4-46: Cyclic polarization curves of PTA Stellite 6 alloy tested in $10 \% \mathrm{H}_{2} \mathrm{SO}_{4} \ldots \ldots \ldots \ldots 133$

Figure 4-47: Cyclic polarization curves of PTA HE6 alloy tested in 10\% $\mathrm{H}_{2} \mathrm{SO}_{4} \ldots \ldots \ldots \ldots \ldots . . . . .134$

Figure 4-48: Comparison of cyclic polarization behavior between PTA Stellite 6 and PTA HE6

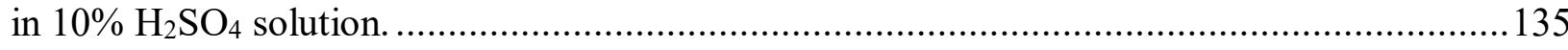

Figure 4-49: Corrosion rates of tested alloys in polarization tests. ................................. 136

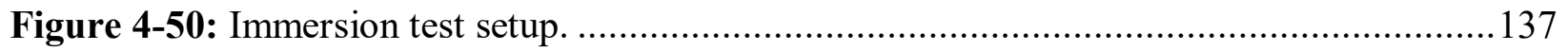

Figure 4-51: Alloy specimen held on a plastic clip. .............................................. 138 
Figure 4-52: Corrosion rate variations with time of the alloys in immersion test.

Figure 4-53: SEM surface morphology of PTA Stellite 6 specimen: (a) as-received, (b) immersion-tested in 5\% HCl solution for 24 hours, (c) immersion-tested in 5\% $\mathrm{HCl}$ solution for 72 hours.

Figure 4-54: EDX analyses of the light region in PTA Stellite 6 specimen surface after immersion tested in $5 \% \mathrm{HCl}$ solution for 72 hours.

Figure 4-55: EDX analyses of the dark region in PTA Stellite 6 specimen surface after immersion tested in $5 \% \mathrm{HCl}$ solution for 72 hours.

Figure 4-56: SEM surface morphology of PTA Stellite 6 immersion-tested in $10 \% \mathrm{H}_{2} \mathrm{SO}_{4}$ solution (a) for 24 hours, (b) for 72 hours.

Figure 4-57: SEM surface morphology of PTA HE6: (a) as-received, (b) immersion-tested in

$5 \% \mathrm{HCl}$ solution for 24 hours, (c) immersion-tested in $5 \% \mathrm{HCl}$ solution for 72 hours. 145

Figure 4-58: SEM surface morphology of PTA HE6 immersion-tested in $10 \% \mathrm{H}_{2} \mathrm{SO}_{4}$ solution for (a) 24 hours and (b) for 72 hours. 146 


\section{List of Acronyms}

AC

ASTM

BSE

CE

CPE

DC

EDX

EIS

FRA

HV

HRC

OCP

PM

PTA

RE

SCE

SEM

SFE

SPS

UNS

WE

XRD
Alternating Current

American Society for Testing and Materials

Backscatter Electron

Counter Electrode

Constant Phase Element

Direct Current

Energy Dispersive X-ray

Electrochemical Impedance Spectroscopy

Frequency Response Analyzer

Hardness Vickers Scale

Hardness Rockwell C Scale

Open Circuit Potential

Powder Metallurgy

Plasma Transferred Arc

Reference Electrode

Saturated Calomel Electrode

Scanning Electron Microscopy

Stacking Fault Energy

Spark Plasma Sintering

Unified Numbering System

Working Electrode

X-ray Diffraction 


\section{Chapter 1: Introduction}

\subsection{Research Background and Significance}

Stellite alloys are a group of cobalt-chromium superalloys consisting of complex carbides and/or intermetallics embedded in a cobalt (Co) solid solution matrix. They combine good mechanical properties with excellent wear resistance, especially at high temperatures, along with admirable oxidation and corrosion resistance in various harsh environments. Their uses span multiple fields among aviation sector, petroleum industry and medical industry, etc.

Stellite alloys are Co-based with additions of chromium (Cr), tungsten (W), carbon (C) and/or molybdenum (Mo). The combination of these elements provides a variety of properties for Stellite alloys in specific applications. Carbon is a critical element for designing a Stellite alloy since varying content of carbon significantly affects the performance of the alloys. The lower carbon alloys are generally recommended for cavitation, sliding wear or moderate galling wear, while the higher carbon alloys are usually selected for abrasion, severe galling, or low angle erosion wear [1].

Two strengthening mechanisms are involved in Stellite alloys, which contribute to their superior properties to other metallic materials. One is solid-solution strengthening and the other is precipitation strengthening [2]. Chemical composition determines the microstructure and properties of Stellite alloys, but the manufacturing process and heat treatment can influence the microstructure and performance of final products significantly [2]. Manufacturing methods of Stellite alloys span a wide range of processes such as numbers of casting techniques, powder metallurgy, welding, 3D printing, and so on. Different processing procedures have great impacts on the microstructural morphology, such as grain size, precipitate type, and crystal structure of the 
solid solution, etc. To choose a proper manufacturing method for a specific application is key to release the great potential of Stellite alloys.

Among the hundreds of Stellite grades, Stellite 6 is the most popular as it provides a good balance of the properties of Stellite alloys, and has become a benchmark of Stellite alloy family. This alloy possesses an excellent combination of mechanical properties, high-temperature performance, wear and corrosion resistance in a wide range of oxidizing and reducing environments which benefit from its unique combination of chemical composition and microstructure [3][4]. Therefore, Stellite 6 alloy is often chosen as a reference alloy for comparison in the studies of Stellite alloys. In the present research, Stellite 6 is also selected for this purpose under the same investigation with the newly developed cobalt high entropy alloy (HE6).

Traditional alloy-design concepts often rely on one or two major components with the minor addition of several other elements to modify the structure and improve overall properties of materials, for example, cobalt-based superalloys, i.e., Stellite alloys. A new concept of alloys, high entropy alloys (HEAs) with multi-components in equiatomic fraction, has been proposed by Dr. Yeh and other pioneer researchers [5] in recent years. High entropy alloy refers to an alloy that consists of five or more principal elements with large mixing of entropies $\left(\Delta S_{\text {conf }} \geq 1.5 R, R\right.$ is gas constant) [6]. The concentration of each principal element can vary between 5 and 35 at.\%, while some minor elements ( $<5$ at.\%) may also participate in the alloy matrix simultaneously. HEAs exhibit various exceptional properties such as high hardness, good ductility and high temperature stability, due to the introduction of extra entropy to stabilize the microstructure [6]. In the new multi-component material design regime, the combination of more than five major elements in one alloy system provides a brand-new perspective, with respect to entropy and 
entropy-related concepts. Combining the power of computational material science, the HEA concept will bring much wider possibilities for new alloy systems in the near future. Since the concept of HEAs came out in 2004, extensive researches have been investigated over the years. Figure 1-1 [7] reveals the increasing number of journal publications related to HEAs suggesting the rising research interest in HEAs.

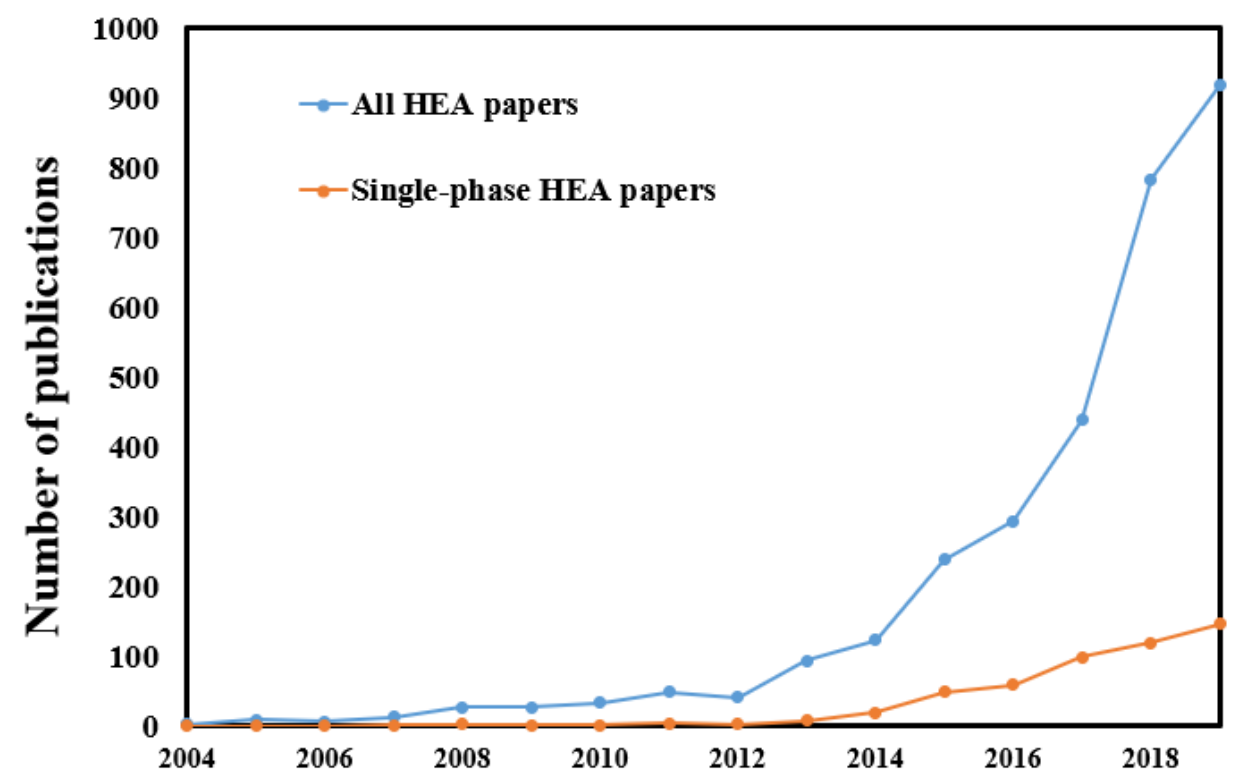

Figure 1-1: Publications related to HEAs [7].

High entropy alloys can essentially be solid solution or amorphous, depending on their chemical composition and have been widely studied around the world [8]-[13]. Due to the mixing elements in equiatomic or near equiatomic compositions, a higher value of entropy can be obtained from HEAs naturally which allows disordered phases to be favored in the alloy matrix. With further research, more HEAs with simple solid solutions, such as face-centered cubic (FCC), bodycentered cubic (BCC) and hexagonal close-packed (HCP), have been invented to obtain excellent mechanical properties [14]-[16], physical properties [15][16], chemical properties [19] and biocompatibility [20]. 
The development of Stellite alloys during their long history has made them popularly used in various environments, especially at elevated temperatures, usually above $540{ }^{\circ} \mathrm{C}$. The outstanding properties of Stellite alloys are largely attributed to the high content (>50 wt.\%) of Co element. Cobalt element plays a crucial role in displaying the exceptional properties of Stellite alloys because the solid solution matrix contains mainly Co with other alloying elements such as $\mathrm{Cr}, \mathrm{W}, \mathrm{Mo}, \mathrm{C}$, etc. Cobalt imparts to its alloys an unstable FCC crystal structure with a very low stacking fault energy (SFE). The unstable FCC structure and its associated low stacking fault energy are believed to result in high yield strength, high work-hardening rate due to the interaction between stacking faults, limited fatigue damage under cyclic stress due to the lack of cell walls within plastically deformed material, the ability to absorb stress through the transformation of the structure to HCP [21]. However, high cost of Stellite alloys due to the limited reserves of cobalt ores worldwide has been a major problem for large use of these alloys. Recently, the industry collaborator, Kennametal Stellite Inc. — a world-wide leading company in producing various superalloys, has proposed a new alloy in terms of the HEA concept, with the aim at reducing Co content in Stellite alloys hence the cost of the alloys, while the performance of the novel HEA can possibly remain at the same level of or even exceed conventional Stellite alloys. Comparing to conventional Stellite alloys, HE6 with much lower Co content can reduce more than $40 \%$ of the material cost.

\subsection{Objectives of Present Research}

In this research, a novel cobalt high entropy alloy, designated as HE6, is created, having near equiatomic Co-Cr-Fe-Ni composition (22 at.\%) with large amounts of $\mathrm{W}$ and small amounts of $\mathrm{C}$ and Mo. Compared with conventional Stellite alloys, the Co content ( $>50$ wt.\%) has been dramatically decreased and other major elements have substituted the spare portion in this new 
alloy. As a result, the cost of raw materials can be greatly reduced. Stellite alloy with lower cost is one of the driving forces of this study.

Due to the loss in the advantages of Co which is the key constituent in Stellite alloys, HE6 alloy is not expected to be comparable to Stellite alloys. However, by utilizing the HEA concept to design the chemical composition of HE6 alloy, it can be predicted that the beneficial features of HEAs may compensate for the loss in superior properties due to reduced Co content. Moreover, this research is also aimed to explore the characteristics of this novel cobalt high entropy alloy and find if this alloy surpasses conventional Stellite alloys in some aspects. Stellite alloys have extensive applications, but they are mainly used for wear and corrosion resistance, such as high pressure valves, bushings, cutting tools, turbine blade hardfacing/repair, etc. Therefore, the novel HEA alloy, HE6, was investigated under hardness, wear and corrosion tests in this research.

Another objective of this research is to employ the advanced manufacturing technique spark plasma sintering (SPS) to create the bulk specimens of HE6 alloy, which is a novel processing method for manufacturing bulk materials through powder metallurgy (PM). Owing to its shorter sintering time and lower heating temperature, compared to other sintering and remelting techniques, SPS has been drawing much attention in producing advanced materials in recent decade. As well known, the manufacturing process of alloys can greatly affect their performance in actual work. Hence, a traditional technique, plasma transferred arc (PTA) welding, is also used in this research to fabricate the bulk alloy specimens in order to compare with SPS processing technique. PTA is a well-developed thermal process for applying tribological and corrosive resistant overlays on substrate materials. Due to its high quality with economical characteristics, PTA has a wide range of commercial uses when hardfacing and repairing metal components are 
needed. Therefore, it is of great significance to investigate the effects of these two processes on the characteristics and properties of the created alloy. As the most popular Stellite alloy and a benchmark in Stellite alloy family, Stellite 6 alloy is under the same investigation with HE6 alloy for comparison in this research.

\subsection{Tasks and Methodologies}

To achieve the goals of this research, the tasks that will be completed with the methods used are outlined below.

(1) Design the chemical composition of the new cobalt high entropy alloy, HE6, in terms of computational entropy theorem.

(2) Examine the raw powders of HE6 and Stellite 6 alloy using a scanning electron microscope (SEM) for particle morphology and size distribution.

(3) Investigate the phase transformation behavior of raw powders of HE6 and Stellite 6 alloy using Differential scanning calorimetry (DSC), which helps understand the SPS and PTA processes for preparing the bulk alloy specimens.

(4) Conduct a density test on the HE6 and Stellite 6 specimens prepared from SPS and PTA processes using the image processing method.

(5) Conduct microstructural analysis on the HE6 and Stellite 6 specimens prepared from SPS and PTA processes using X-ray diffraction (XRD), SEM and energy-dispersive X-ray spectroscopy (EDX), focusing on microstructure morphology, phases present in the microstructure, and carbide/intermetallic volume fraction of each specimen. 
(6) Conduct hardness test on the HE6 and Stellite 6 specimens prepared from SPS and PTA processes using a Microhardness Tester (HV scale), Model SMT-X7 Dual Indenter and a Rockwell Hardness Tester (HRC scale).

(7) Conduct dry-sliding wear test on the HE6 and Stellite 6 specimens prepared from SPS and PTA processes using a NEO-TRIBO Model MPW110 Pin-on-Disc Wear Test System, in order to evaluate the wear loss and friction coefficient of each specimen.

(8) Examine the worn surfaces of the HE6 and Stellite 6 specimens using SEM to understand the wear mechanism of the materials, according to the morphology changes of the specimen surface.

(9) Conduct electrochemical corrosion test on the HE6 and Stellite 6 specimens prepared from PTA process in $3.5 \% \mathrm{NaCl}, 2 \% \mathrm{HCl}$ and $10 \% \mathrm{H}_{2} \mathrm{SO}_{4}$ solutions to investigate the polarization behavior of the materials in the solutions.

(10) Conduct immersion test on the HE6 and Stellite 6 specimens prepared from PTA process in $2 \% \mathrm{HCl}$ and $10 \% \mathrm{H}_{2} \mathrm{SO}_{4}$ solutions to evaluate the corrosion rate of the materials in the solutions for different exposure times.

(11) Analyze the surface morphology of the specimens from the immersion tests with $\mathrm{SEM} / \mathrm{EDX}$ to investigate the corrosion mechanism of the alloys in $2 \% \mathrm{HCl}$ and $10 \% \mathrm{H}_{2} \mathrm{SO}_{4}$ solutions and better understand the corrosion rate results.

(12) Analyze the results of hardness, wear and corrosion tests with respect to the microstructures of the HE6 and Stellite 6 specimens, discuss the effects of SPS and PTA 
manufacturing processes on the microstructure evolution of HE6 and Stellite 6 alloy, justify the significance of developing cobalt high entropy alloys.

(13) Discuss the cost reduction of HE6 according to high costs of Stellite alloys and potential applications of HE6.

\subsection{Organization of the Thesis}

Five chapters are written for this thesis to cover the entire work of the present research. The main contents that are included in each chapter are outlined below.

Chapter 1 is a brief introduction to the present research, including the background and significance, objectives, tasks and methodologies, of this research. The structure of this thesis is also introduced in this chapter.

Chapter 2 gives a brief literature review of Stellite alloys and high entropy alloys including their microstructures and various properties. Different manufacturing processes and performance evaluation methods for these alloys are also reviewed in this chapter.

Chapter 3 details the experimental work on Stellite 6 and HE6 raw powders, including SEM morphology examination and investigation of particle size distribution, as well as DSC analysis. The detailed microstructural analyses of bulk HE6 and Stellite 6 specimens from SPS and PTA processes, using XRD/SEM/EDX, and the density measurement of the bulk specimens, are also described in this chapter.

Chapter 4 delineates all the experiments for performance evaluation of bulk HE6 and Stellite 6 specimens from SPS and PTA processes, including hardness, dry-sliding wear, 
electrochemical and immersion corrosion tests. The discussion on the experimental results, in terms of the relationship between the microstructure and material properties of the alloys, is also included in this chapter.

Chapter 5 summarizes this research and draws the conclusions from this research. The significant contributions of this research are highlighted and the future work of this research is recommended. 


\section{Chapter 2: Literature Review}

\subsection{Stellite Alloys}

Superalloys or high-performance alloys are designed to work at high temperatures (above $\sim 500^{\circ} \mathrm{C}$ ). Several key characteristics of superalloys include excellent mechanical strength, resistance to thermal creep deformation, good surface stability, and resistance to corrosion or oxidation, etc. [22].

Stellite alloy is a group of cobalt-chromium-tungsten (Co-Cr-W) or cobalt-chromiummolybdenum (Co-Cr-Mo) superalloy composed of complex carbides embedded in a cobalt solid solution matrix. It is designed mainly for high wear resistance and excellent corrosion properties in harsh environments. The combination of cobalt and chromium also has a high melting point, making it ideal for a range of interesting applications, from cutting tools to hot zone coatings in gas turbines [2], which involve high temperature.

During the past 100 years, Stellite alloys have been used in various industries, such as aerospace, oil and gas, medical, and so on. Owing to the outstanding efforts of the metallurgists, these superalloys have been widely developed for crucial applications, for example, blades and vanes in aircraft engines, nuclear valves, etc. [2]. Stellite alloys are mainly composed of transition metals, which have continuous positions in the periodic table. Another characteristic of these alloys is that they are generally strengthened by solid solution and precipitation hardening mechanisms [23].

As a group of Co-based superalloys designed for wear and corrosion resistance applications, the main alloying elements in a Stellite alloy system are chromium $(\mathrm{Cr})$, tungsten (W), molybdenum (Mo) and carbon (C). The balance of these elements in the alloy will provide 
various sorts of properties to meet the needs of a specific application. The common composition of Stellite alloy is that they usually contain $40-60 \% \mathrm{Co}, 20-30 \% \mathrm{Cr}, 5-15 \% \mathrm{~W}$ or Mo, and 0.1 - 3\% C (in weight percentage), as shown in Table 2-1 [2]. One of the main differences between these alloys is the contents of $\mathrm{C}, \mathrm{W}$ and Mo, hence the volume fraction of various carbides precipitated in the alloys [2].

Table 2-1: Chemical compositions (wt.\%) of Stellite alloys [2].

\begin{tabular}{|c|c|c|c|c|c|c|c|c|c|c|c|}
\hline Alloy & Process & Co & $\mathrm{Cr}$ & $\mathrm{W}$ & $\mathrm{Mo}$ & $\mathrm{C}$ & $\mathrm{Fe}$ & $\mathrm{Ni}$ & $\mathrm{Si}$ & $\mathrm{Mn}$ & Others \\
\hline Stellite 1 & Cast & 47.7 & 30 & 13 & 0.5 & 2.5 & 3 & 1.5 & 1.3 & 0.5 & \\
\hline Stellite 3 & P/M & 49.8 & 30.5 & 12.5 & & 2.3 & 1.8 & 1.8 & 0.7 & 0.6 & \\
\hline Stellite 4 & Cast & 45.43 & 30 & 14 & $1^{*}$ & 0.57 & $3^{*}$ & $3^{*}$ & $2^{*}$ & $1^{*}$ & \\
\hline Stellite 6 & Cast & 55.3 & 29 & 4.5 & $1.5^{*}$ & 1.2 & $3^{*}$ & $3^{*}$ & $1.5^{*}$ & $1^{*}$ & \\
\hline Stellite 6 & P/M & 51.5 & 28.5 & 4.5 & $1.5^{*}$ & 1 & $5^{*}$ & $3^{*}$ & $2^{*}$ & $2^{*}$ & $1 \mathrm{~B}^{*}$ \\
\hline Stellite 12 & Cast & 52.6 & 30 & 8.3 & & 1.4 & 3 & 1.5 & 0.7 & 2.5 & \\
\hline Stellite 21 & Cast & 59.5 & 27 & & 5.5 & 0.25 & $3^{*}$ & 2.75 & $1^{*}$ & $1^{*}$ & \\
\hline Stellite 20 & Cast & 41.05 & 33 & 17.5 & & 2.45 & 2.5 & 2.5 & & 1 & \\
\hline Stellite 22 & Cast & 54 & 27 & & 11 & 0.25 & 3 & 2.75 & 1 & 1 & \\
\hline Stellite 25 & Cast & 49.4 & 20 & 15 & & 0.1 & 3 & 10 & 1 & 1.5 & \\
\hline Stellite 31 & Cast & 57.5 & 22 & 7.5 & & 0.5 & 1.5 & 10 & 0.5 & 0.5 & \\
\hline Stellite 80 & Cast & 44.6 & 33.5 & 19 & & 1.9 & & & & & $1 \mathrm{~B}$ \\
\hline Stellite 188 & Cast & 37.27 & 22 & 14 & & 0.1 & 3 & 22 & 0.35 & 1.25 & $0.03 \mathrm{La}$ \\
\hline Stellite 190 & Weld & 46.7 & 27 & 14 & 1 & 3.3 & 3 & 3 & 1 & 1 & \\
\hline Stellite 300 & Cast & 44.5 & 22 & 32 & & 1.5 & & & & & \\
\hline Stellite 694 & Cast & 45 & 28 & 19 & & 1 & 5 & & 1 & 1 & \\
\hline Stellite 712 & Cast & 51.5 & 29 & & 8.5 & 2 & $3^{*}$ & $3^{*}$ & $1.5^{*}$ & $1.5^{*}$ & \\
\hline Stellite 720 & Cast & 37.2 & 33 & & 18 & 2.5 & $3^{*}$ & $3^{*}$ & $1.5^{*}$ & $1.5^{*}$ & $0.3 \mathrm{~B}$ \\
\hline Stellite 703 & Cast & 44.6 & 32 & & 12 & 2.4 & $3^{*}$ & $3^{*}$ & $1.5^{*}$ & $1.5^{*}$ & \\
\hline Stellite 706 & Cast & 55.8 & 29 & & 5 & 1.2 & $3^{*}$ & $3^{*}$ & $1.5^{*}$ & $1.5^{*}$ & \\
\hline
\end{tabular}




\begin{tabular}{|c|c|c|c|c|c|c|c|c|c|c|c|}
\hline Stellite 706K & Wrought & 54.6 & 29 & & 6 & 1.4 & $3^{*}$ & $3^{*}$ & $1.5^{*}$ & $1.5^{*}$ & \\
\hline Stellite 728 & Cast & 55.88 & 24 & & 12 & 0.35 & 1 & 3.8 & 0.45 & 0.52 & $2 \mathrm{Nb}$ \\
\hline Stellite 6B & Wrought & 55.9 & 30 & 4 & $1.5^{*}$ & 1 & $3^{*}$ & 2.5 & 0.7 & 1.4 & \\
\hline Stellite 6K & Wrought & 52.4 & 30 & 4.5 & $1.5^{*}$ & 1.6 & $3^{*}$ & $3^{*}$ & $2^{*}$ & 2 & \\
\hline Stellite 98M2 & P/M & 36.2 & 30 & 18.5 & $0.8^{*}$ & 2 & $5^{*}$ & 3.5 & $1^{*}$ & $1^{*}$ & $\begin{array}{c}4.2 \mathrm{~V}, 1 \\
\mathrm{~B}^{*}\end{array}$ \\
\hline
\end{tabular}

(* indicates the max. content of the certain element in alloys)

\subsubsection{Alloying elements and precipitates of Stellite alloys}

Cobalt has a hexagonal crystal form (HCP) at room temperature, and can change to face centered cubic (FCC) form at a higher temperature (above $400^{\circ} \mathrm{C}$ ). When a cobalt alloy is cooled slowly, Co transforms from FCC to $\mathrm{HCP}$ at $417^{\circ} \mathrm{C}$, which means that the alloy is in a metastable state [24]. Due to the sluggish nature of the transformation, the FCC Co structure of its alloys is usually preserved at room temperature, and the formation of HCP is stimulated only by mechanical stress or contact at elevated temperatures. The FCC to HCP transformation of Co will result in high yield strength, high work-hardening rate of the alloys due to the interaction between stacking faults and the ability to relax stress [25], [26].

Carbon content in Stellite alloys plays an important role in the microstructure and type of carbides formed in these alloys. It is a key enhancer for the wear resistance of Stellite alloys. For instance, at a carbon content of 2.4 wt.\% (for Stellite 3), carbides constitute about $30 \mathrm{wt} \%$ of the material. These carbides belong to $\mathrm{M}_{7} \mathrm{C}_{3}\left(\mathrm{Cr}\right.$-rich primary) and $\mathrm{M}_{6} \mathrm{C}$ (W-rich eutectic) types, where M represents metallic elements. For Stellite 6B, carbides constitute approximately $13 \mathrm{wt} . \%$ of the material, with the carbon content of $1 \mathrm{wt. \%}$ [2]. For all Stellite alloys, when the carbon content is less than 2 wt.\%, these alloys are hypoeutectic, and FCC cobalt dendrites are surrounded by eutectic $\mathrm{M}_{7} \mathrm{C}_{3}$ carbides. When the carbon content is greater than $2.5 \mathrm{wt} . \%$, they are hypereutectic 
and consist of primary $\mathrm{M}_{7} \mathrm{C}_{3}$ carbides in the dendritic eutectic matrix, including Co solid solution and carbides. Other carbides formed in hypereutectic Stellite alloys include W-rich $\mathrm{M}_{6} \mathrm{C}$ and $\mathrm{MC}$, and $\mathrm{Cr}$-rich $\mathrm{M}_{23} \mathrm{C}_{6}$ [27]. Figure 2-1 shows some typical microstructures of Stellite alloys, which were all from laser cladding process. The coatings of Stellite 6 and Stellite 12 have a hypoeutectic microstructure that consists of primary dendrites of Co solid solution surrounded by eutectic carbides, while Stellite 1 has a hypereutectic microstructure composed of primary carbides dispersed in a eutectic interdendritic matrix of Co solid solution and carbides.

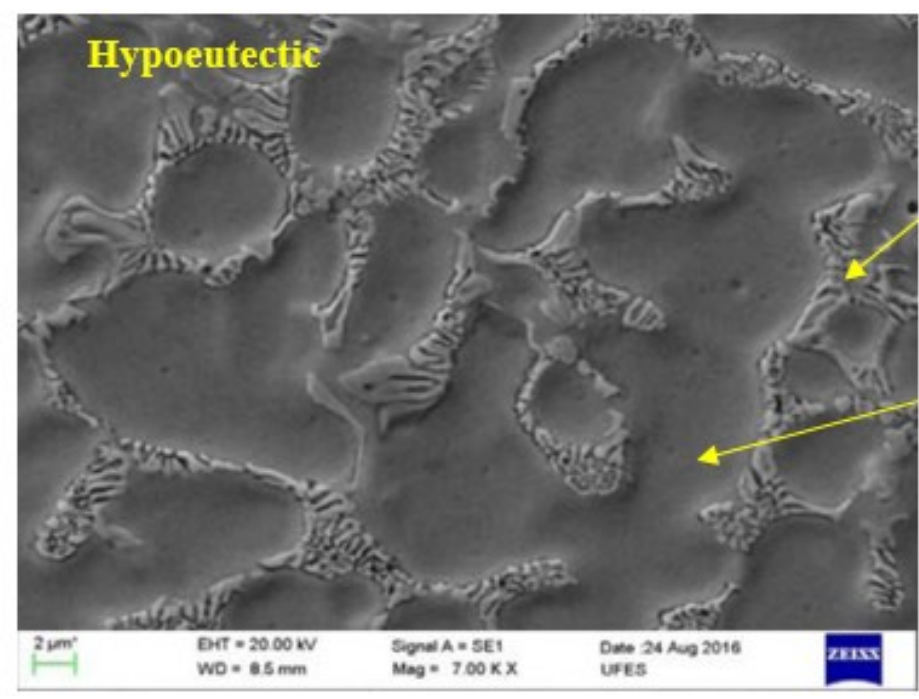

(a)

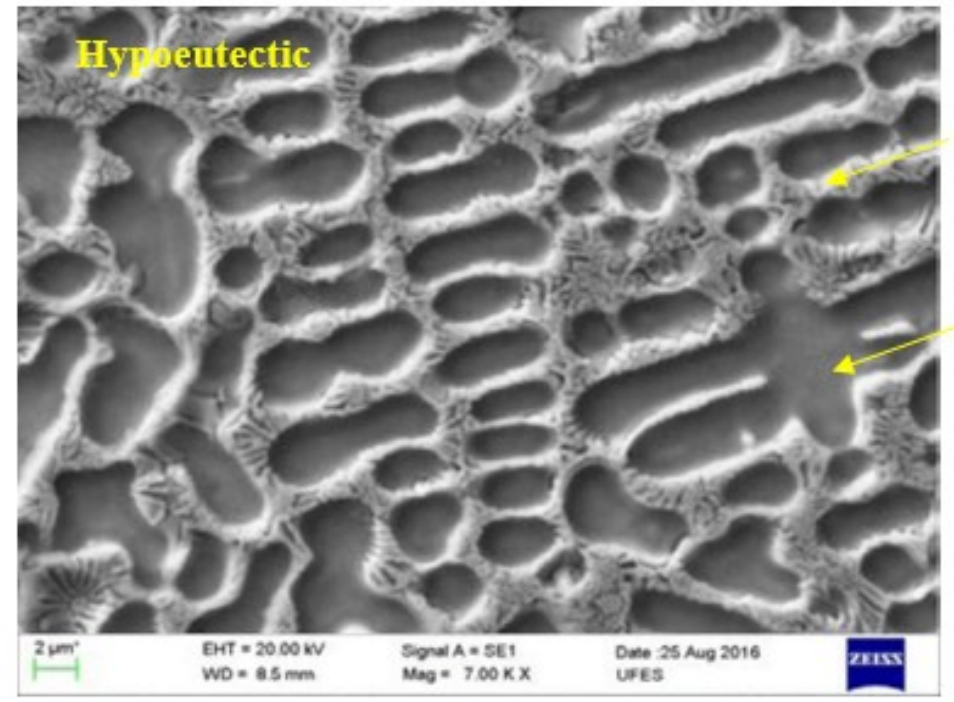

(b)
Eutectic carbide

Dendritic Co-based solid solution

Eutectic carbide

Dendritic Co-based solid solution 


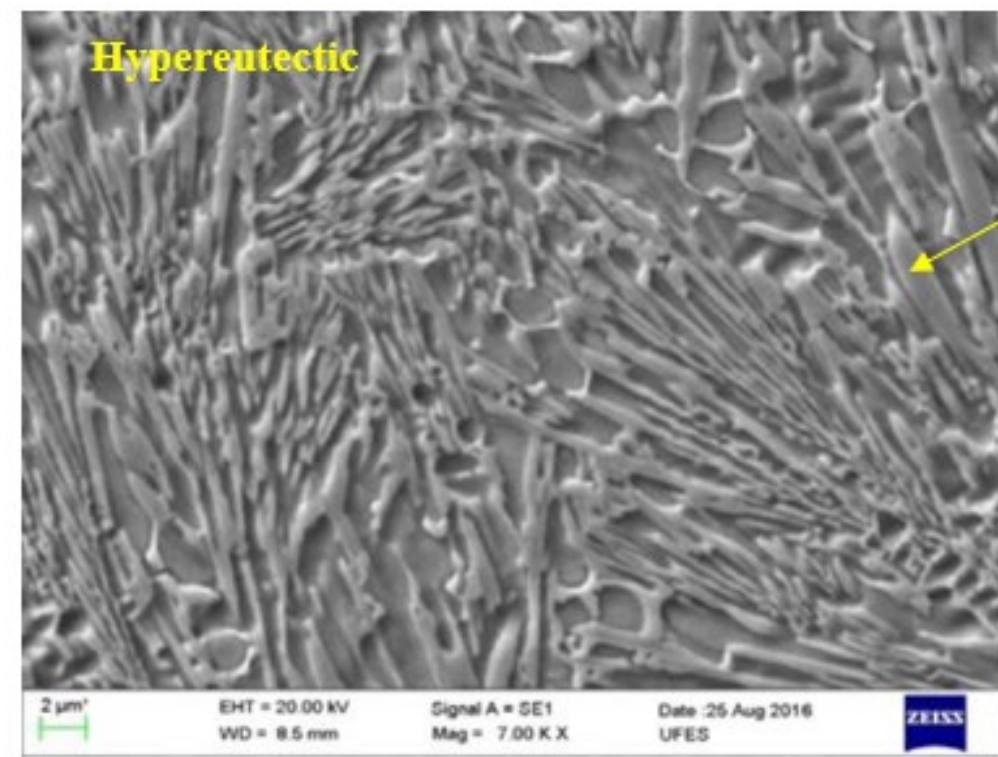

\section{Primary carbide}

(c)

Figure 2-1: Typical microstructure of laser cladding of (a) Stellite 6, (b) Stellite 12, (c) Stellite 1 [28].

Chromium in Stellite alloy has a dual function. it is not only a major carbide former, that is, the carbides in Stellite alloys are mainly Cr-rich, but also crucial in the solid solution matrix which provides strengthening. The most common type of carbides in Stellite alloys is Cr-rich $\mathrm{M}_{7} \mathrm{C}_{3}$, while $\mathrm{Cr}$-rich $\mathrm{M}_{23} \mathrm{C}_{6}$ carbides are also abundant in low-carbon grades. At the same time, $\mathrm{Cr}$ imparts favorable characteristics to the alloys, such as oxidation resistance and corrosion resistance to the oxidizing environment [29], [30].

Tungsten and molybdenum in Stellite alloys provide additional strengthening to the solid solution matrix due to their large atomic size as solutes [30], thus dislocation flow can be prevented. They also improve the overall corrosion resistance of the alloys [2], [25]. Moreover, W and Mo usually participate in carbide formation when present in large quantities.

There are also other alloying elements used in Stellite alloys, such as nickel (Ni), iron (Fe), aluminum (Al), boron $(\mathrm{B})$, carbon $(\mathrm{C})$, manganese $(\mathrm{Mn})$, silicon $(\mathrm{Si})$, titanium $(\mathrm{Ti})$, etc. The proportion of these alloying elements varies, and most grades contain four to six elements. For 
instance, the addition of alloying elements of $\mathrm{Ni}, \mathrm{Cu}$ and $\mathrm{Fe}$ will improve the stability of FCC, but will increase the stacking fault energy (SFE) of Co-rich solution matrix. In contrast, $\mathrm{Cr}$, Mo and W provide the stability of HCP at low temperatures due to decreasing the SFE [31].

\subsubsection{Previous research on Stellite 6 alloy}

Stellite 6 alloy is a benchmark in the family of Stellite alloys. Due to its great performance in severe conditions, it becomes one of the most widely used Stellite alloys. Consequently, there have been abundant studies on this alloy during the years. In this thesis, Stellite 6 has been chosen to compare with HE6 alloy.

Stellite 6, which is from a casting process, can be altered into Stellite 6B and Stellite 6K, which are wrought products. The carbon content of Stellite $6 \mathrm{~K}(1.6 \mathrm{wt} . \% \mathrm{C})$ is higher than that of Stellite 6B (1 wt.\% C), which results in differences in their properties and applications. Stellite 6B has both excellent wear resistance and toughness, also good workability. The applications of Stellite 6B involve high-impact pulp agitators, critical directional drilling tools, and aerospace components. Stellite 6K has similar properties to Stellite 6B but it is slightly harder and less ductile. Stellite $6 \mathrm{~K}$ is excellent for cutting or scraping applications, such as knives or scraper blades.

In 2003, Malayoglu and Neville conducted a comparative study in the corrosive performance of Stellite 6 and two stainless steels, UNS S32760 and UNS S31603, in liquid-solid states [32]. Figure 2-2 shows that the material losses in $3.5 \% \mathrm{NaCl}$ medium of the cast and HIPed Stellite 6 are consistently lower than that of stainless steels in the erosion-corrosion test. The results suggest that Stellite 6 has superior erosion and corrosion resistance compared to superduplex (UNS S32760) and austenitic (UNS S31603) stainless steel. 

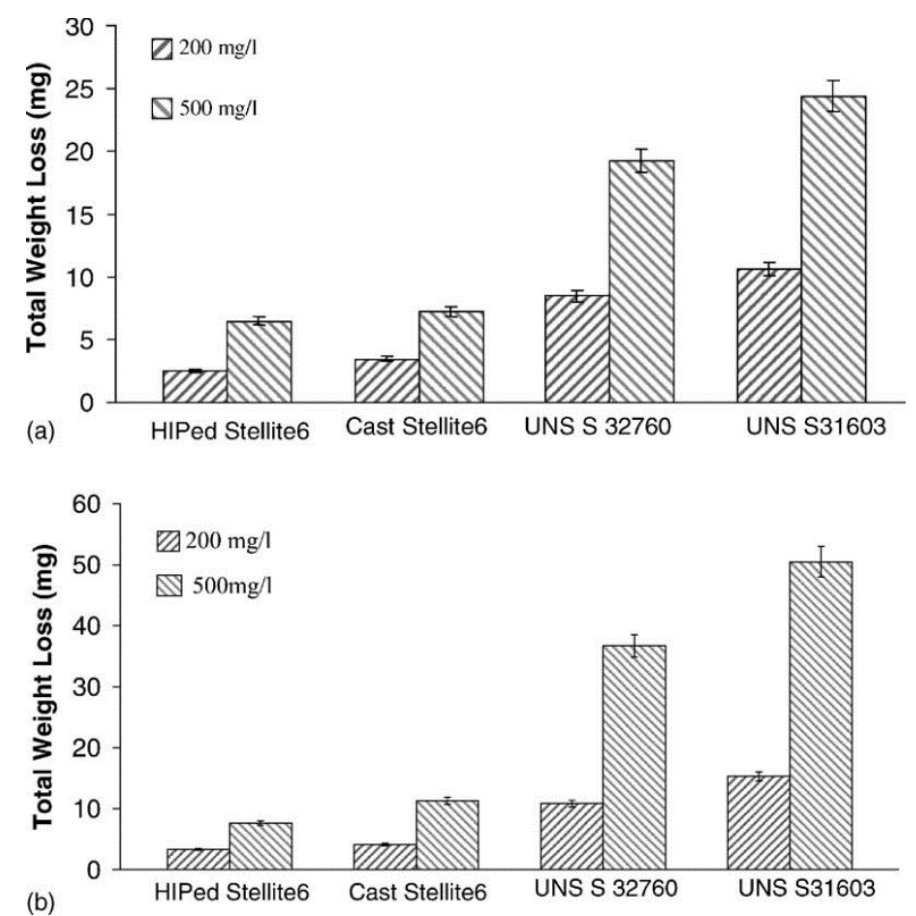

Figure 2-2: Total weight loss measurements of Stellite alloys and stainless steels in $3.5 \% \mathrm{NaCl}$ solution at (a) $18^{\circ} \mathrm{C}$ and (b) $50^{\circ} \mathrm{C}$.

Wear and corrosion resistance of $\mathrm{W}$ or Mo containing Stellite alloy hardfacings were investigated by Yao et al. in 2005 [33]. A series of tests were conducted to discover the abrasive, adhesive and erosive wear resistance of these hardfacings. As shown in Figure 2-3, Mo-containing Stellite alloys (Stellite 706, Stellite712 and Stellite 790) have higher abrasive resistance than Wcontaining Stellite alloys (Stellite 6, Stellite 12 and Stellite 190) under both low and high-stress abrasive-wear conditions. The corrosion rates of Stellite 6, Stellite 706, Stellite 12 and Stellite 712 are summarized in Figure 2-4. It can be seen that W-containing Stellite alloys (Stellite 6 and Stellite 12) have better corrosion resistance in $10 \% \mathrm{HNO}_{3}$ acid at boiling temperature, while Mocontaining Stellite alloys (Stellite 706 and Stellite 712) exhibit exceptional corrosion resistance in $5 \% \mathrm{HCl}$ acid at $40^{\circ} \mathrm{C}$ and $10 \% \mathrm{H}_{2} \mathrm{SO}_{4}$ at $66^{\circ} \mathrm{C}$. 


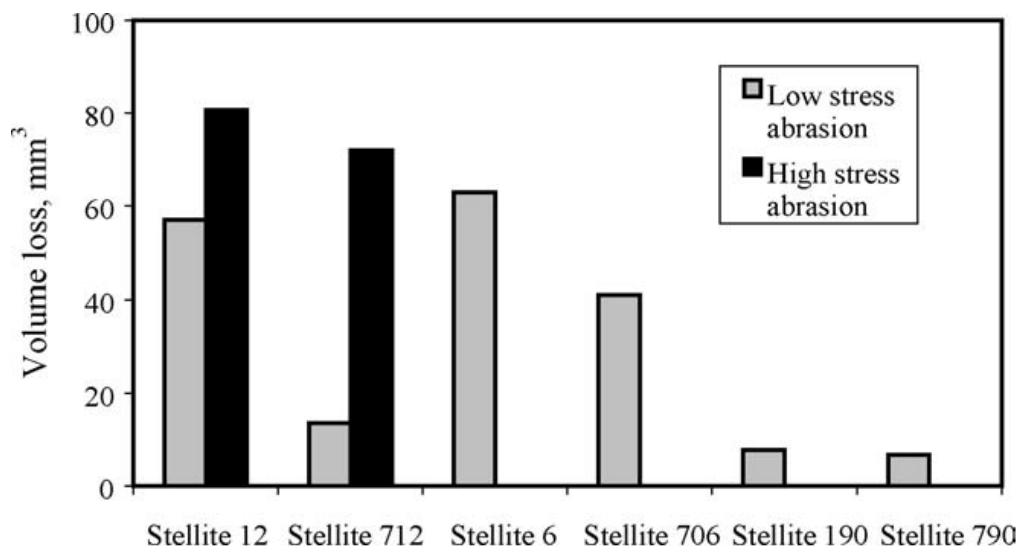

Figure 2-3: Low-stress abrasive and high-stress abrasive-wear resistance of Stellite alloys[33][33][33][33][33][33][33][33][33].

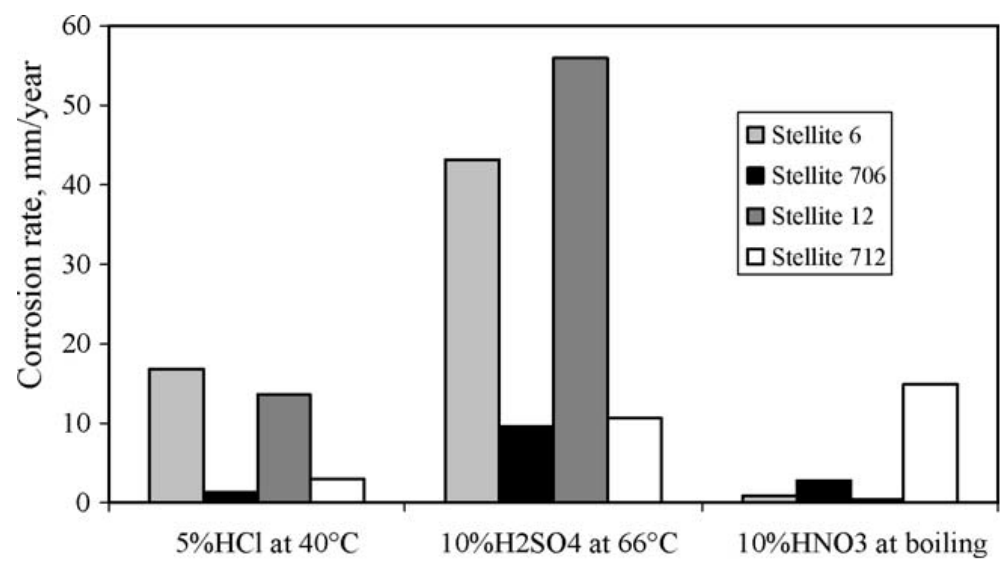

Figure 2-4: Corrosion resistance of W-containing Stellite alloys and Mo-containing Stellite alloys. 


\subsection{High Entropy Alloys}

High entropy alloys are defined as alloys containing at least five principal elements, each with an atomic percentage between 5 and $35 \%$. Also, they are alloys with configurational entropies larger than $1.5 R$, where $R$ is the gas constant, $8.314 \mathrm{~J} / \mathrm{K} / \mathrm{mol}[6]$.

\subsubsection{Origin and definition}

Three independent publications by Yeh in Taiwan [5], Cantor in the United Kingdom in 2004 [34], and Ranganathan in India in 2003 [35], took the lead in exploring the high entropy alloy world. A brand-new concept, "high entropy alloys (HEAs)" or "multi-principal element alloys (MPEAs)" was brought into reality and investigated through increased scientific studies. This new concept placed a new milestone in the history of alloy development. During the following decade, extensive researches have been conducted world-widely, as mentioned in Chapter 1:.

To better understand the definition of HEAs, an understanding of the mixing of entropy and configurational entropy of alloys is needed. The total mixing entropy has four contributions: configurational, vibrational, magnetic dipole, and electronic randomness. Configurational entropy is dominant over the other three contributions [5]. Hence, the configurational entropy often represents the mixing entropy to avoid complicated calculations to determine the other three contributions.

From statistical thermodynamics, Boltzmann's Equation [36], [37] calculates the configurational entropy $\left(\Delta S_{\text {conf }}\right)$ of a system:

$$
\Delta S_{\text {conf }}=k_{B} \ln w
$$

where $k_{B}$ is Boltzmann's constant, and $w$ is the number of ways in which the available energy can be mixed or shared among the particles in the system. For a random $n$-component solid solution, in which the $i$ th component has a mole fraction $X_{i}$, the ideal configurational entropy per mole is: 


$$
\Delta S_{\text {conf }}=-R \sum_{i=1}^{n} X_{i} \ln X_{i}
$$

where $R$ is the gas constant, $8.314 \mathrm{~J} / \mathrm{K} / \mathrm{mol}$.

Based on the earlier concepts, there are two definitions for HEAs [6]. One is based on composition, and the other is according to configurational entropy. For the former, HEAs are defined as the alloys containing five to thirteen principal elements with an atomic percentage between 5 and 35\%. The atomic portion of each minor element is less than 5\%. This definition can be expressed as

$$
13 \geq n_{\text {major }} \geq 5,5 \% \leq X_{i} \leq 35 \% \text { and } n_{\text {minor }} \geq 0 X_{j} \leq 5 \%
$$

where $n_{\text {major }}$ and $n_{\text {minor }}$ are the number of major elements and minor elements, respectively. $X_{i}$ and $X_{j}$ are the atomic fraction of major element $i$ and minor element $j$, respectively.

For the later, HEAs are also defined as alloys having configurational entropies at a random state larger than $1.5 R[6]$, which can be expressed as:

$$
\Delta S_{\text {conf }} \geq 1.5 R
$$

However, these two definitions are not laws but they are general guidelines for alloy classifications. The compositions out of the defined region can be treated as HEAs as well. For instance, $\mathrm{Co}_{29.4} \mathrm{Cr}_{29.4} \mathrm{Cu}_{5.9} \mathrm{Fe}_{5.9} \mathrm{Ni}_{29.4}$ is considered as a HEA that fits into the first definition, but its configurational entropy is approximately $1.414 \mathrm{R}$ which does not fit into the high entropy definition. As for the quaternary equimolar alloy $\mathrm{Co}-\mathrm{Cr}-\mathrm{Fe}-\mathrm{Ni}$, it is sometimes considered as a HEA in literature because its composition and configurational entropy are close to the lower limits of both definitions. 
From the two general definitions of HEAs, the basic principle behind HEAs with multiple principal elements is to have high mixing entropy in order to promote the formation of solid solution phase and inhibit the formation of intermetallic compounds [6].

Since $1.5 R$ is a lower limit for HEAs, there is also a classification of medium-entropy alloys (MEAs) and low-entropy alloys (LEAs) to differentiate the power of mixing entropy effect, i.e.,

$$
\begin{gathered}
\text { MEAs: } 1.0 \mathrm{R} \leq \Delta S_{\text {conf }} \leq 1.5 R \\
\text { LEAs: } \Delta S_{\text {conf }} \leq 1.0 R
\end{gathered}
$$

Table 2-2 reports the configurational entropies calculated for some well-known steels/alloys at their liquid state [38]. It is shown that most steels/alloys have low or medium entropy. Furthermore, some single-principal-element alloys such as Ni-based, Co-based superalloys, and bulk metallic glass (BMGs) have medium entropy. Also, some Stellite alloys are chosen to calculate their entropies and the results are given in Table 2-3.

Table 2-2: Entropy values calculated for typical traditional alloys at their liquid state or random state [38].

\begin{tabular}{c|c|c}
\hline Systems & Alloys & Value of Entropy \\
\hline \multirow{2}{*}{ Low-alloy steel } & 4340 & $0.22 R$ low \\
\hline \multirow{2}{*}{ Stainless steel } & 304 & $0.96 R$ low \\
\cline { 2 - 3 } & 316 & $1.15 R$ medium \\
\hline High-speed steel & $\mathrm{M} 2$ & $0.73 R$ low \\
\hline \multirow{2}{*}{ Mg alloy } & AZ91D & $0.35 R$ low \\
\hline \multirow{2}{*}{ Al alloy } & 2024 & $0.29 R$ low \\
\cline { 2 - 3 } & 7075 & $0.43 R$ low \\
\hline \multirow{2}{*}{ Ni-based superalloy } & $7-3$ brass & $0.61 R$ low \\
\cline { 2 - 3 } & Inconel $718_{18}$ & $1.31 R$ medium \\
\hline \multirow{2}{*}{ BMG } & $\mathrm{Hastelloy} \mathrm{X}$ & $1.37 R$ medium \\
\cline { 2 - 3 } & $\mathrm{Cu}_{47} \mathrm{Zr}_{11} \mathrm{Ti}_{34} \mathrm{Ni}_{8}$ & $1.17 R$ medium \\
\cline { 2 - 3 } & $\mathrm{Zr}_{53} \mathrm{Cu}_{16} \mathrm{Ni}_{10} \mathrm{Al}_{16}$ &
\end{tabular}


Table 2-3: Entropy values calculated for typical Stellite alloys.

\begin{tabular}{|c|c|c|c|c|c|c|c|c|c|c|c|c|c|}
\hline \multirow{2}{*}{ Grade } & \multirow{2}{*}{$\begin{array}{c}\text { Entropy } \\
(\Delta \mathbf{S})\end{array}$} & \multirow{2}{*}{ Classification } & \multicolumn{11}{|c|}{ Composition } \\
\hline & & & & Co & $\mathrm{Cr}$ & $\mathbf{W}$ & Mo & $\mathbf{F e}$ & $\mathbf{N i}$ & Mn & $\mathbf{C}$ & $\mathbf{S i}$ & B \\
\hline \multirow{2}{*}{ Stellite 21} & \multirow{2}{*}{$1.17 \mathrm{R}$} & \multirow{16}{*}{$\begin{array}{c}\text { MEA } \\
(\mathbf{1 . 5}>\Delta S \geq 1)\end{array}$} & wt.\% & 59.50 & 27.00 & 0.00 & 5.50 & 3.00 & 2.75 & 1.00 & 0.25 & 1.00 & 0.00 \\
\hline & & & at.\% & 57.30 & 29.50 & 0.00 & 3.26 & 3.04 & 2.65 & 1.03 & 1.18 & 2.03 & 0.00 \\
\hline \multirow{2}{*}{$\begin{array}{c}\text { Tribaloy } \\
\text { T-400 }\end{array}$} & \multirow{2}{*}{$1.19 \mathrm{R}$} & & wt.\% & 56.62 & 8.50 & 0.00 & 29.00 & 1.50 & 1.50 & 0.00 & 0.08 & 2.80 & 0.00 \\
\hline & & & at.\% & 60.58 & 10.32 & 0.00 & 19.07 & 1.69 & 1.60 & 0.00 & 0.42 & 6.31 & 0.00 \\
\hline \multirow{2}{*}{ Stellite 12} & \multirow{2}{*}{$1.30 \mathrm{R}$} & & wt. $\%$ & 52.60 & 30.00 & 8.30 & 0.00 & 3.00 & 1.50 & 2.50 & 1.40 & 0.70 & 0.00 \\
\hline & & & at.\% & 50.09 & 32.42 & 2.53 & 0.00 & 3.01 & 1.43 & 2.55 & 6.56 & 1.40 & 0.00 \\
\hline \multirow{2}{*}{ Stellite 6} & \multirow{2}{*}{$1.32 \mathrm{R}$} & & wt.\% & 55.30 & 29.00 & 4.50 & 1.50 & 3.00 & 3.00 & 1.00 & 1.20 & 1.50 & 0.00 \\
\hline & & & at.\% & 51.75 & 30.79 & 1.35 & 0.86 & 2.96 & 2.81 & 1.00 & 5.52 & 2.96 & 0.00 \\
\hline \multirow{2}{*}{$\begin{array}{c}\text { Stellite } \\
706 \\
\end{array}$} & \multirow{2}{*}{$1.33 \mathrm{R}$} & & wt.\% & 55.80 & 29.00 & 0.00 & 5.00 & 3.00 & 3.00 & 1.50 & 1.20 & 1.50 & 0.00 \\
\hline & & & at.\% & 31.38 & 30.30 & 0.00 & 2.83 & 2.90 & 2.76 & 1.48 & 5.43 & 2.91 & 0.00 \\
\hline \multirow{2}{*}{ Stellite 25} & \multirow{2}{*}{$1.37 \mathrm{R}$} & & wt. $\%$ & 49.40 & 20.00 & 15.00 & 0.00 & 3.00 & 10.00 & 1.50 & 0.10 & 1.00 & 0.00 \\
\hline & & & at.\% & 52.40 & 24.07 & 5.10 & 0.00 & 3.35 & 10.61 & 1.71 & 0.52 & 2.24 & 0.00 \\
\hline \multirow{2}{*}{ Stellite 1} & \multirow{2}{*}{$1.40 \mathrm{R}$} & & wt. \% & 47.70 & 30.00 & 13.00 & 0.50 & 3.00 & 1.50 & 0.50 & 2.50 & 1.30 & 0.00 \\
\hline & & & at.\% & 44.81 & 31.98 & 3.92 & 0.29 & 2.97 & 1.41 & 0.50 & 11.55 & 2.57 & 0.00 \\
\hline \multirow{2}{*}{$\begin{array}{c}\text { Stellite } \\
712\end{array}$} & \multirow{2}{*}{$1.44 \mathrm{R}$} & & wt. $\%$ & 51.50 & 29.00 & 0.00 & 8.50 & 3.00 & 3.00 & 1.50 & 2.00 & 1.50 & 0.00 \\
\hline & & & at.\% & 46.65 & 29.81 & 0.00 & 4.73 & 2.86 & 2.72 & 1.46 & 8.91 & 2.86 & 0.00 \\
\hline \multirow{2}{*}{$\begin{array}{c}\text { Stellite } \\
720\end{array}$} & \multirow{2}{*}{$1.63 \mathrm{R}$} & \multirow{2}{*}{$\begin{array}{c}\text { HEA } \\
(\Delta S \geq 1.5)\end{array}$} & wt. $\%$ & 37.20 & 33.00 & 0.00 & 18.00 & 3.00 & 3.00 & 1.50 & 2.50 & 1.50 & 0.30 \\
\hline & & & at. $\%$ & 33.65 & 33.87 & 0.00 & 10.01 & 2.86 & 2.71 & 1.46 & 11.12 & 2.86 & 1.46 \\
\hline
\end{tabular}

\subsubsection{Essential parameters and formation rules}

Certainly, an alloy must be designed and created on scientific basis, therefore some criteria are needed to quantify the alloy properties, in order to justify the feasibility of the alloy. As a result, some parameters, such as configurational entropy, the enthalpy of mixing have been developed.

1 Configurational entropy

As described in the previous section, the configurational entropy of an alloy is calculated by Equation 2.2 using the atomic content of each element and gas constant $R$.

2 The enthalpy of mixing

The enthalpy of mixing for an alloy is calculated by: 


$$
\Delta H_{\text {mix }}=4 \sum_{i=1, i=j}^{n} \Delta H_{i, j}^{m i x} X_{i} X_{j}
$$

where $\Delta H_{i, j}^{\text {mix }}$ is the enthalpy of mixing between element $i$ and $j$ in the liquid state with equimolar compositions taken from the table edited by Takeuchi et al. [39], based on Miedema's model [39], and $n$ is the total number of elements in the solution phase.

3 Atomic size difference [6]

Atomic size difference measures the composition-weighted difference in the atomic radii among constituent elements of an alloy and is calculated by:

$$
\begin{gathered}
\delta=\sqrt{\sum_{i=1}^{n} X_{i}\left(1-r_{i} / \bar{r}\right)^{2}} \\
\bar{r}=\sum_{i=1}^{n} X_{i} r_{i}
\end{gathered}
$$

where $r_{i}$ is the atomic radius of the $i$ th element.

4 Parameter $\Omega[6]$

Parameter $\Omega$ correlates the melting point, total entropy of mixing, and the total enthalpy of mixing as:

$$
\Omega=\frac{T_{m} * \Delta S_{m i x}}{\left|\Delta H_{m i x}\right|}
$$

where $T_{m}$ is the average melting temperature of the alloy, and $\Delta S_{m i x}$ and $\Delta H_{m i x}$ are the total entropy of mixing and the enthalpy of mixing in the liquid phase, respectively. Both enthalpy and entropy contribute to Gibbs-free energy. Therefore, this ratio can reflect the entropy and enthalpy effects and reveal which of them has more influence on the alloy at the melting point. This ratio implies that when $\Omega \geq 1$, the entropy effect at $T_{m}$ is greater than the enthalpy effect, hence the high 
entropy solid solution phase tends to form. The higher the value, the stronger the high entropy impact is. Thus, it is more likely that random solid solution gains stability to survive at low temperatures.

5 Valence electron concentration (VEC)

The value of VEC can be defined as the number of total electrons, including the d-electrons accommodated in the valence band, valence electron concentration or VEC [40], [41]. VEC for a multi-component alloy can be defined as the weighted average from the VEC of the constituent components:

$$
\mathrm{VEC}=\sum_{i=1}^{n} X_{i}(V E C)_{i}
$$

where $(V E C)_{i}$ are the VEC value for the individual element [42].

In the designing process, VEC can be used to quantitatively predict the phase stability for FCC and BCC portion in HEAs: in the research work of Guo et al. [42], at VEC $\geq 8.0$, single FCC phase existed; at $6.87 \leq \mathrm{VEC}<8.0$, mixed $\mathrm{FCC}$ and $\mathrm{BCC}$ phases co-existed, and single $\mathrm{BCC}$ phase existed at VEC $<6.87$.

After checking through a large number of alloys, including solid solution alloys, intermetallic compounds, and BMG, Zhang et al. [9] firstly proposed an empirical rule for forming HEAs; if a combination of $-15 \leq \Delta H_{m i x} \leq+5 \mathrm{~kJ} / \mathrm{mol}$ and $\delta \leq 5 \%$ was valid, then disordered solid solution phase was favored. An additional rule was proposed by Zhang et al. [43] that a combination of $\Omega \geq 1$ and $\delta \leq 6.6 \%$ also favored disordered solid solution formation. 


\subsubsection{Fabrication methods}

HEAs are essentially solid solution alloys, containing many major elements with simple crystal structures, such as BCC, FCC and HCP lattices. The typical manufacturing routes of traditional materials can be applied to HEAs. According to the mixing methods of constituent elements, the processes are divided into liquid melting, solid-state mechanical alloying plus an additional sintering, and gaseous mixing, as illustrated in Figure 2-5 [6]. The first route starts in the liquid state and includes arc melting, resistance melting, induction melting, laser melting, laser cladding and laser enhanced mesh forming (LENS). The second method comes from the solidstate and mainly involves mechanical alloying (MA), then followed by the sintering process to fabricate the bulk material. The elements can also be mixed in a vaporized state and the routes include sputtering deposition, pulsed laser deposition (PLD), atomic layer deposition (ALD), molecular beam epitaxy (MBE), and vapor deposition to prepare a film on a substrate. In the present research, plasma transferred arc (PTA) welding and spark plasma sintering (SPS) were utilized to create the HEA bulk specimens.

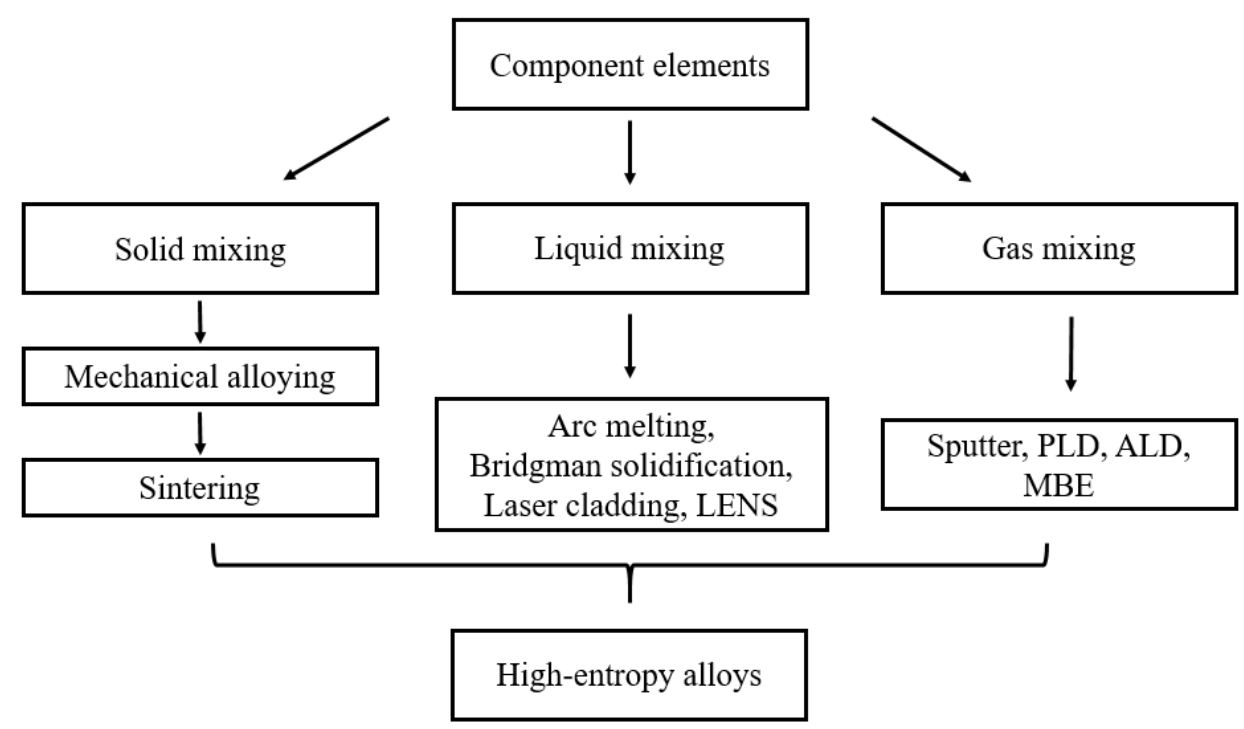

Figure 2-5: Summary of the fabricating routes for HEAs [6]. 


\subsubsection{Plasma transferred arc (PTA) welding}

PTA welding is a thermal process that applies additional layers on the surface of the substrate material. It is a common method for depositing high-quality reinforced top-surface on a variety of base materials. Figure 2-6 [44] schematically shows the working principle of PTA. The welding process uses a contracted high-energy plasma arc between the non-consumable electrode and the substrate to form a molten pool. The filling material can be in the form of welding wire and then fed to the back of the welding pool. The metallurgical bonding layer produced by this method has better fusion and corrosion-resistant properties than that from mechanically bonded processes [44]. In the reported work by Cheng et al. [44], the Co-Cr-Cu-Fe-Ni HEA coatings were successfully prepared by PTA cladding process. The experimental results demonstrated that the coating was entirely an FCC solid solution phase. The microstructure of the coating was mainly composed of dendrite and discontinuous interdendritic segregation.

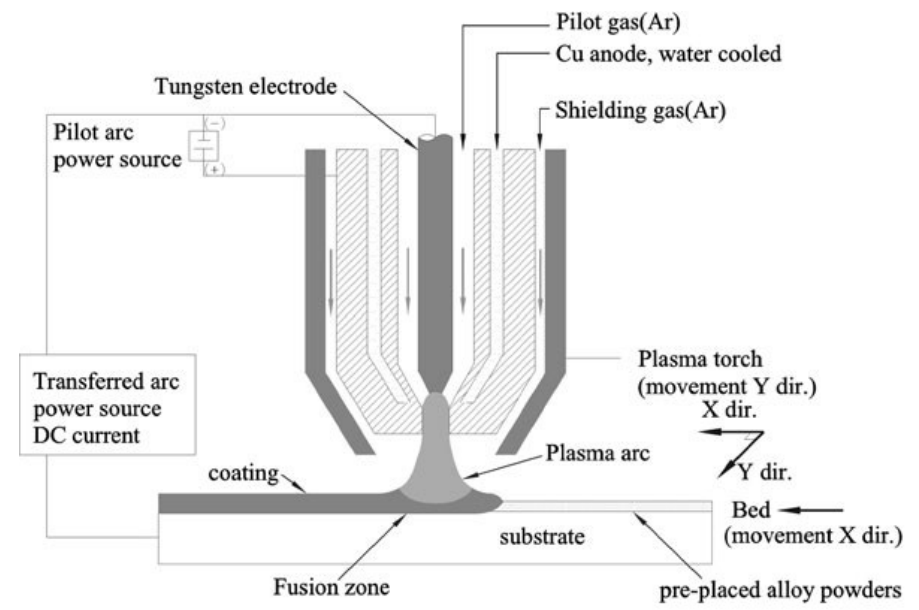

Figure 2-6: Schematic diagram of plasma transferred arc (PTA) process [44]. 


\subsubsection{Spark plasma sintering (SPS)}

SPS is similar to the conventional hot pressing process because the precursor is loaded into a graphite die, and a uniaxial pressure is applied during the sintering process. However, instead of using an external heating source, a pulsed direct current is allowed to pass through the conductive mold. This means that the die is also used as a heating source, and the sample is heated from both outside and inside, as shown in Figure 2-7 [45]. Different from PTA process, SPS is a solidus/liquidus processing method with lower working temperature, with elemental diffusion being the main mechanism of metallurgical bonding between powder particles. In addition to the Joule heat of hot-press sintering and the plastic deformation caused by pressing to promote the sintering process, the SPS process also generates a DC pulse voltage between the powder particles and effectively uses the self-heating feature caused by the discharge between the powder particles [46]. This method produces a relatively high heating rate with a short holding time to obtain fully dense bulk parts.

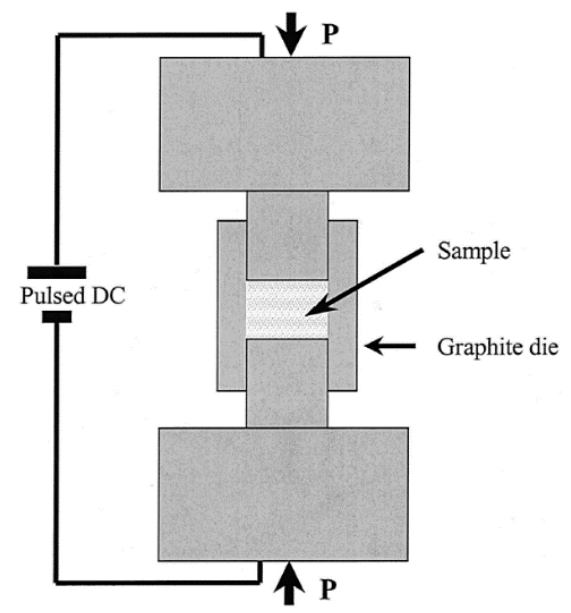

Figure 2-7: Schematic illustration of the working principle of an SPS apparatus [45]. 
Fu et al. [47] studied the effect of Cr additions on the alloying behavior and structure of CoFeNiAl $0.5 \mathrm{Ti}_{0.5}$ prepared by MA and SPS. Praveen et al. [48] reported the phase evolution and densification behavior of nanocrystalline HEAs prepared via MA and compacted with spark plasma sintering (SPS). Their research results showed that multi-component HEAs have been successfully synthesized by these processes.

\subsubsection{Material properties}

HEAs have been widely studied in mechanical and functional performance since 2004 and many HEAs have been reported to display promising properties, such as high hardness, high yielding strengths, high ductility, excellent wear and corrosion resistance.

\subsubsection{Hardness}

Hardness is one common criterion to describe the mechanical properties of metallic materials [49]. Figure 2-8 [6] reports the hardness values of the twenty most studied HEAs, compared with conventional alloys. The value of hardness varies largely within the alloy systems, strongly depending on the chemical composition, fabrication method, and heat-treatment process, etc. For example, the hardness value varies from $154 \mathrm{HV}$ to $658 \mathrm{HV}$ for the AlCoCrCuFeNi alloy system due to the different content of element Al [50]-[53]. In general, researchers are seeking HEAs that have higher hardness than conventional alloys. 


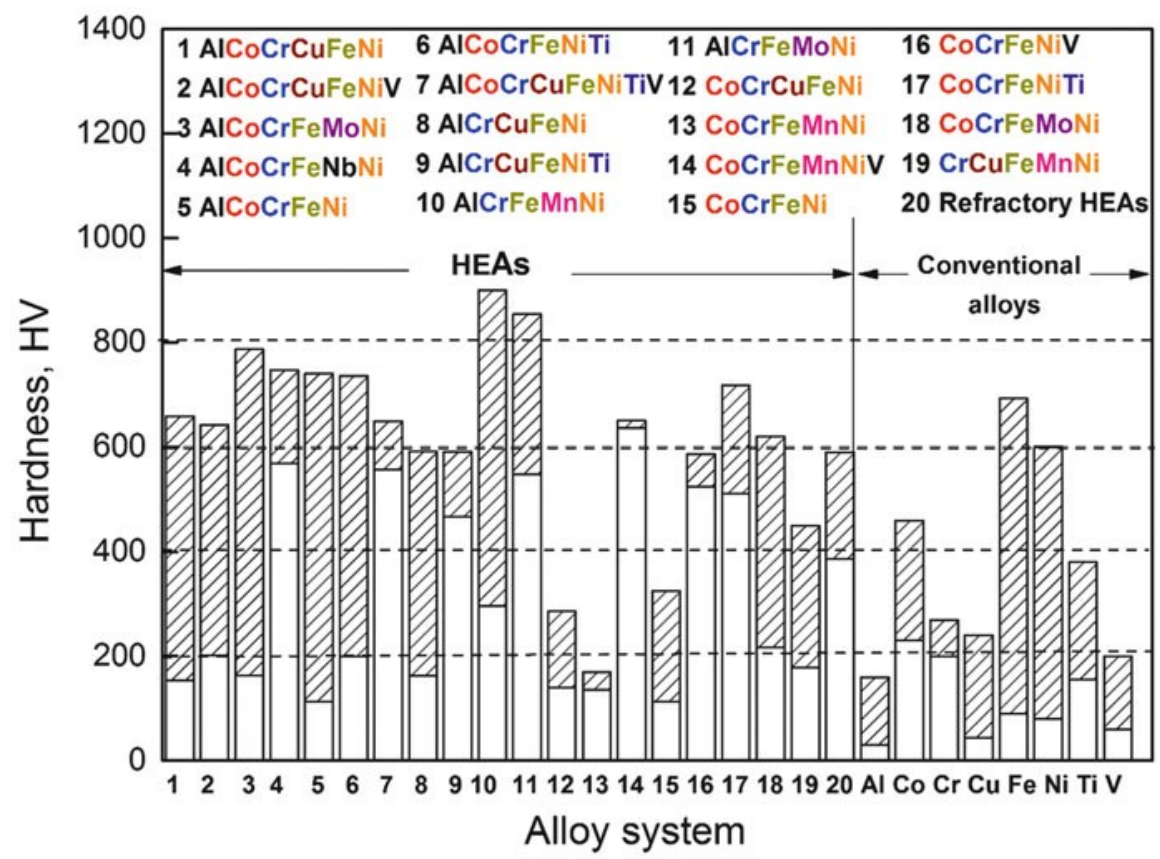

Figure 2-8: Hardness values of most studied HEAs, compared with conventional alloys [6].

\subsubsection{Yield strength and ductility}

Knowledge of the yield strength is critical when designing components since it usually represents the upper limit of the load that can be applied to the material. Having higher toughness implies that the material is capable of withstanding unforeseen loading for a more extended time [6].

Ductility is the ability of a material to be drawn or plastically deformed without fracture. It is an essential factor in allowing a structure to survive extreme loads [6].

HEAs display a wide range of yield strength and ductility at room temperature, as shown in Figure 2-9 [6]. The tensile yield stresses of $\mathrm{AlCoCrCuFeNi}$ and $\mathrm{Al}_{0.5} \mathrm{CoCrCuFeNi}$ are superior to Ti-6Al-4V and Inconel 713. Also, CoCrFeNi alloy has better ductility than conventional 304 stainless steel, Ti-6Al-4V, Inconel 713, and 5083 Al. 


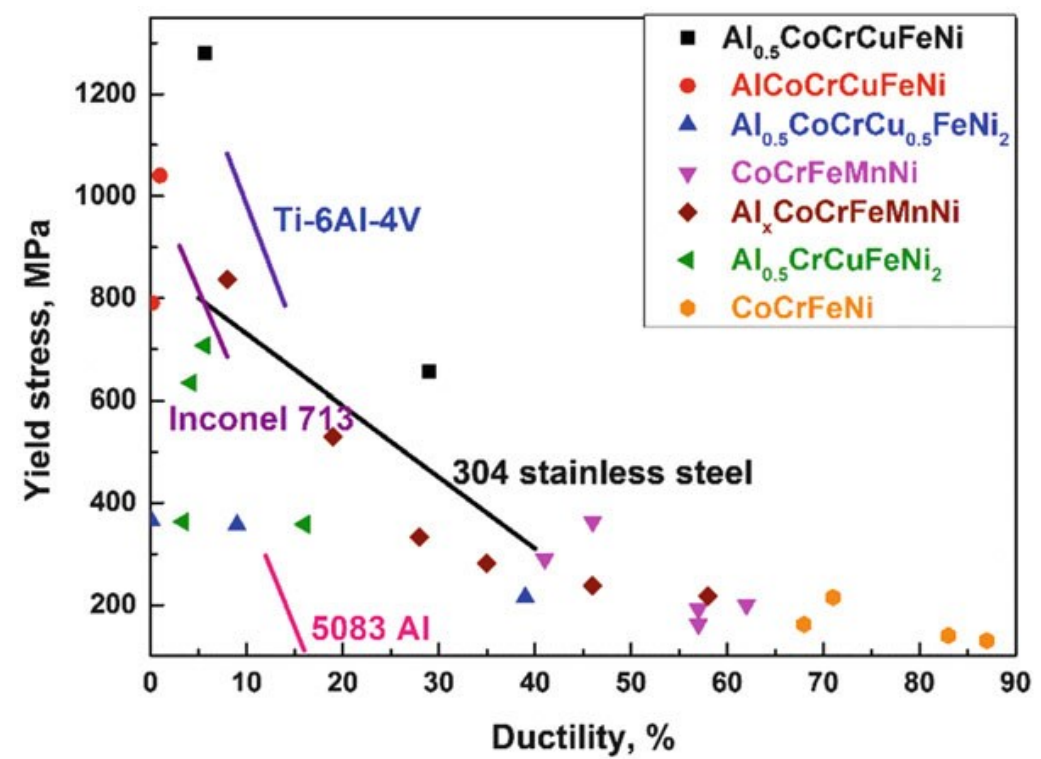

Figure 2-9: Tensile yield stress versus ductility of most studied HEAs at room temperature, compared with conventional alloys [6].

\subsubsection{Wear resistance}

Wear resistance is defined as the ability of a material to resist comprehensive external forces such as abrasion, edge cutting, and impact, etc. during service. It represents the performance of a material to withstand surface damages under contact loads in relative motion. Therefore, the requirements for wear resistance are extremely important in material design [6].

Chuang et al. [54] compared the hardness and wear resistance of HEAs $\left(\mathrm{Al}_{\mathrm{x}} \mathrm{Co}_{1.5} \mathrm{CrFeNi}_{1.5} \mathrm{Ti}_{\mathrm{y}}\right)$ with conventional wear-resistant steels (SUJ2 and SKH51). The results in Figure 2-10 show that A102Ti10 (the abbreviation of $\mathrm{Al}_{0.2} \mathrm{Co}_{1.5} \mathrm{CrFeNi}_{1.5} \mathrm{Ti}_{1.0}$ ) has the best wear resistance among the six alloys, about twice that of Al00Ti10 (the abbreviation of $\mathrm{Al}_{0.0}$ $\left.\mathrm{Co}_{1.5} \mathrm{CrFeNi}_{1.5} \mathrm{Ti}_{1.0}\right)$. The wear resistance of A102Ti10 is much higher than that of the two conventional steels, 3.6 and 2 times higher than that of SUJ2 and SKH51, respectively. This indicates that A102Ti10 has outstanding anti-wear properties. 


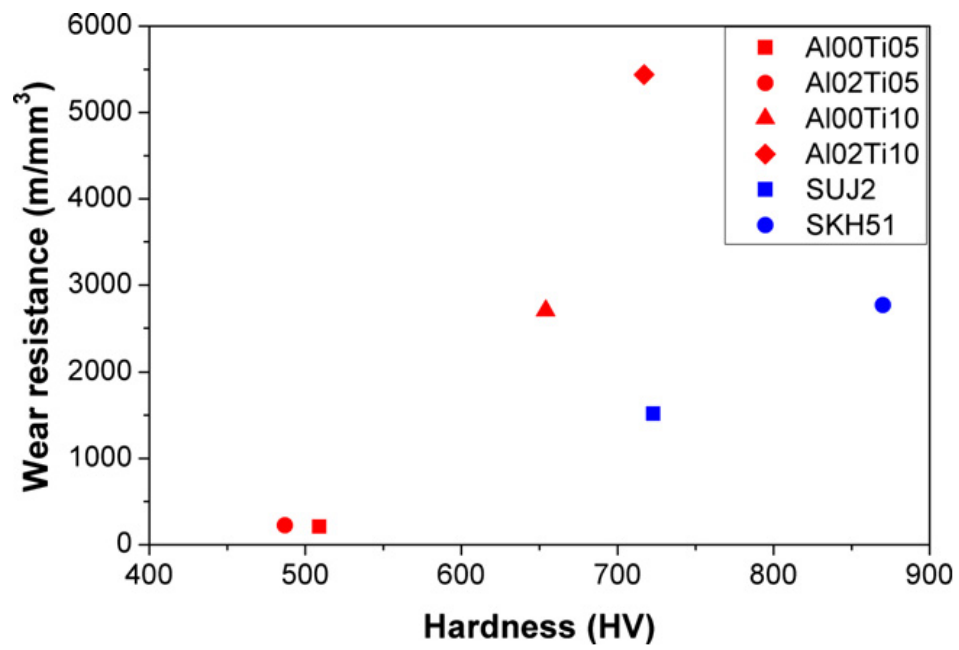

Figure 2-10: Pin-on-disk adhesive wear resistance versus hardness of HEAs and Steels [54].

\subsubsection{Corrosion resistance}

Corrosion is the loss of metals due to a reaction to the environment. Stainless steel, copperbased alloys and nickel-based alloys are designed for various corrosive environments.

Tang et al. [55] compared the corrosion rates of various types of materials such as HEAs and stainless steels in $3.5 \mathrm{wt} . \% \mathrm{NaCl}$ solution and $0.5 \mathrm{M} \mathrm{H}_{2} \mathrm{SO}_{4}$ solution at room temperature, as illustrated in Figure 2-11 and Figure 2-12. The corrosion rate varies significantly among different types of materials and also varies greatly among HEAs. Therefore, it is believed that certain compositions with a proper processing method can render HEAs good corrosion-resistant alloy candidates. 


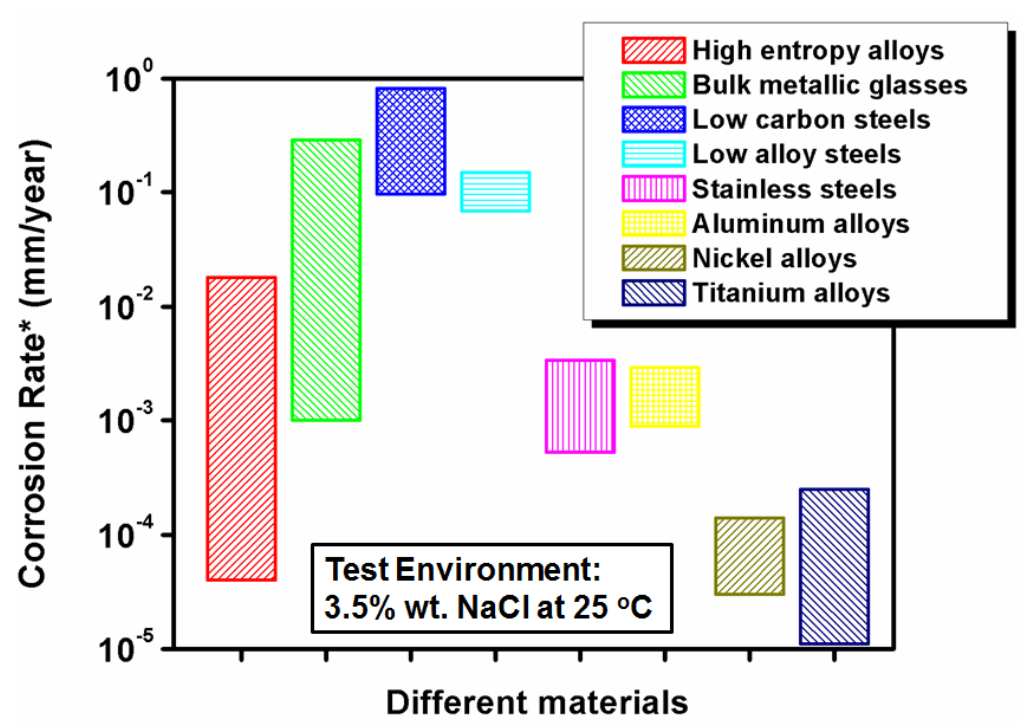

Figure 2-11: A comparison to average corrosion rates (mm/year) between HEAs and other materials in $3.5 \mathrm{wt} \% \mathrm{NaCl}$ solution at room temperature.

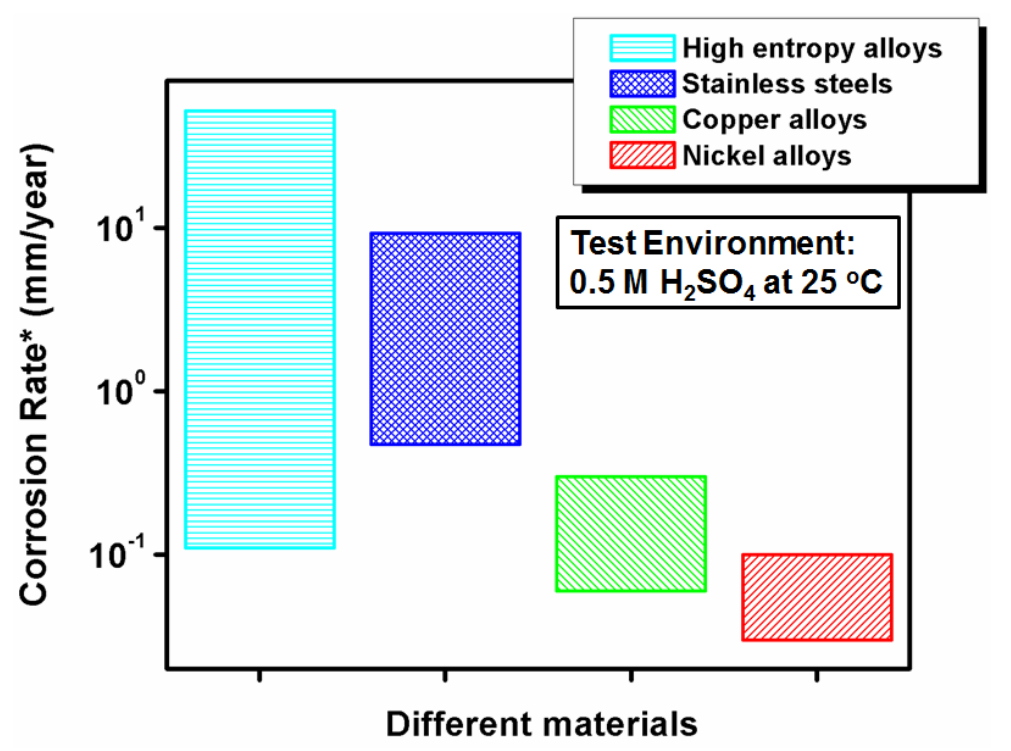

Figure 2-12: A comparison in average corrosion rates ( $\mathrm{mm} /$ year) between HEAs and conventional alloys in $0.5 \mathrm{M} \mathrm{H}_{2} \mathrm{SO}_{4}$ solution at room temperature. 


\subsection{Material Performance Testing Methods}

Stellite alloys were developed for applications where require excellent wear and corrosion resistance. Therefore, it is essential to conduct wear and corrosion experiments on selected Stellite alloy and the new HEA in this study and provide some understandings with respect to such criteria.

\subsubsection{Wear test}

\subsubsection{Wear of materials}

Wear is a process that involves the interactions between surfaces and, more specifically, the removal and deformation of material from a surface as a result of mechanical action of the contacting object through relative motion [56]. The study of wear and related processes is referred to as tribology. Wear occurring on the parts of various machines, together with other procedures such as fatigue and creep, causes functional surfaces to degrade, eventually leading to material failure or loss of functionality. Therefore, wear has high economic relevance so that industries are eager to avoid it [57].

Wear resistance is the ability of materials to withstand surface damage in working conditions. Wear occurs in numerous applications, for example, cables, medical implants (hip joints), clutches, pump clutches, abrasion of clothing, etc. Parts under wear are often visually inspected to assess the wear losses of the part materials. Quantitative evaluation methods for wear are widely used, such as measuring volume change or mass change after the piece is exposed to predetermined wear conditions [58].

\subsubsection{Pin-on-disk wear test}

Wear is commonly classified according to so-called wear types, which occurs in isolation or complex interaction. Common types of wear include adhesive wear, abrasive wear, surface 
fatigue, fretting wear, etc. In reality, wear is caused by one or more wear mechanisms. For example, the primary wear mechanism of adhesive wear is adhesion. Wear mechanisms or submechanisms often overlap and occur in a coordinated manner, resulting in a wear rate greater than the sum of wear rate by the individual wear mechanisms [59].

Adhesive wear and abrasive wear are modes of wear that occur under plastic contact. In the case of plastic contact between similar materials, the contact interface has adhesive bonding strength. When it is assumed that the fracture is basically due to strong adhesion at the contact interface, the resulting wear is called adhesion wear. As a result, adhesive wear occurs when the atomic force between the surfaces of a material is stronger than the inherent material properties of either surface. The continuous movement of the surface can cause the bonded joint to break. Adhesive wear is characterized by one surface's particles sticking to another surface through molecular forces, which results in moving parts stopping abruptly, i.e., failure, as illustrated in Figure 2-13 [60].
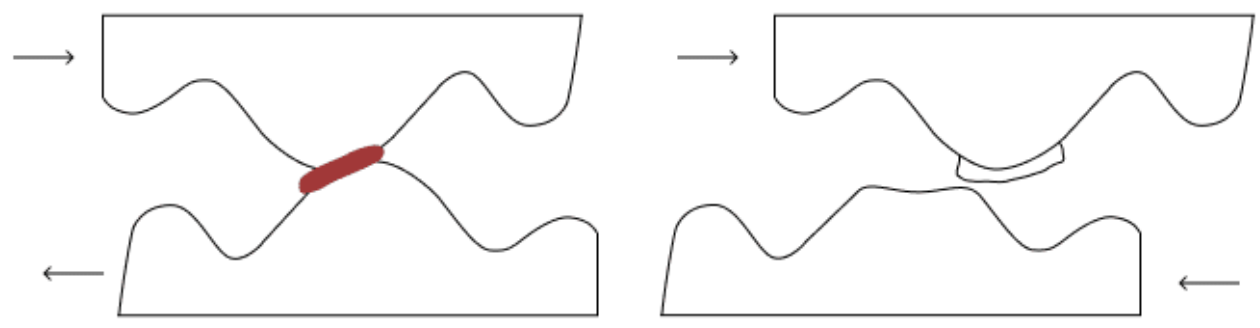

Figure 2-13: Mechanism of adhesive wear of materials [60].

Adhesive wear can be found between surfaces during frictional contact and generally refers to unwanted displacement and attachment of wear debris and material compounds from one surface to another. The deforming effect of small particles or surface protrusions during friction creates abrasive wear. It happens when one material is harder than the other, while the softer one 
is the casualty [61]. Figure 2-14 illustrates the abrasive wear of materials, with three main mechanisms described below:

- Cutting - removal of material (like lathing)

- Fragmentation - a consequence of cutting, cracks forming and further wear breaking off pieces

- Plowing - displacing material to the sides in the direction of scraping

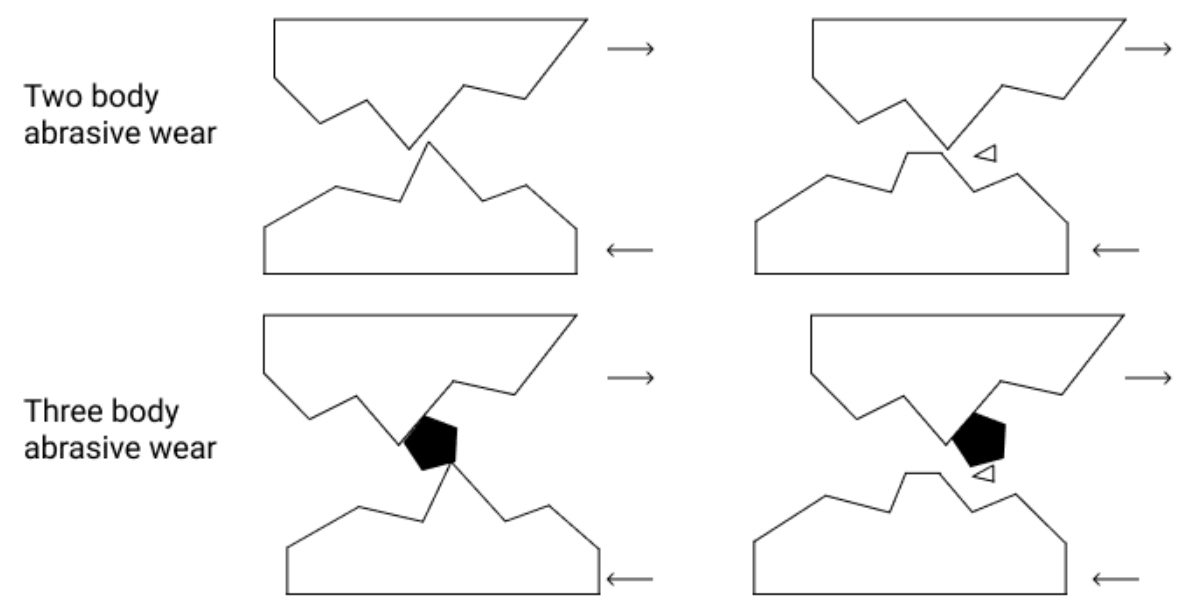

Figure 2-14: Mechanism of abrasive wear in materials [61].

Friction and wear (typically wear rate and wear resistance) characterization of materials is typically performed using various types of tribometers, but pin-on-disk or ball-on-disk test is a common technique. Figure 2-15 schematically illustrates the principle of a pin-on-disk test. The popularity of this method is due to its relative simplicity and abundance of the tribological contacts that can be well described by a simple pin-on-disk motion. The test typically allows performing in several motion modes, such as unidirectional, fretting wear and recently other complex motion patterns. Usually, pin-on-disc tests are conducted according to ASTM Standard G99 - 17[62]. 


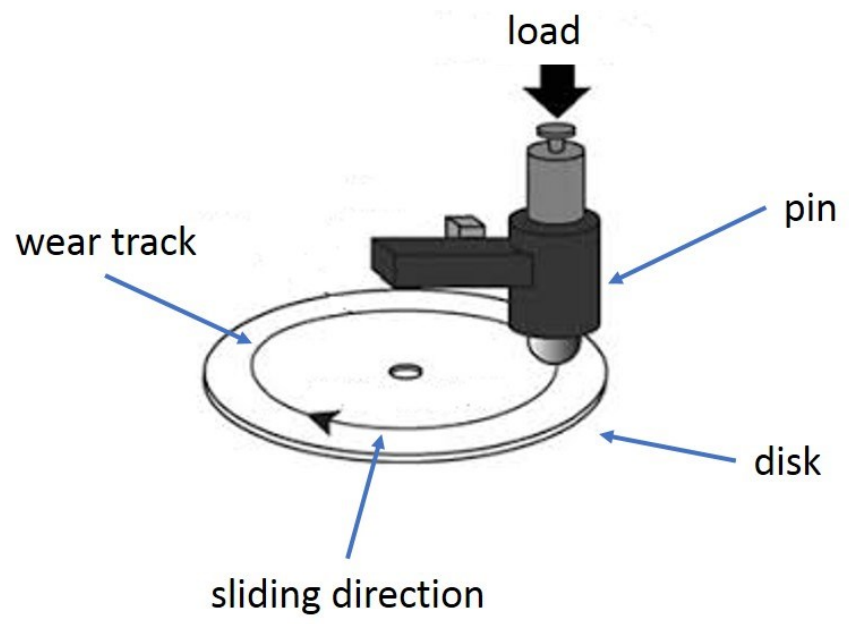

Figure 2-15: Schematic diagram of a pin-on-disk test [63].

\subsubsection{Related researches in wear test}

Birol et al. [64] conducted a study in wear performance comparison between three different alloys under a ball-on-disc sliding wear test. Figure 2-16 reports the volume losses of the three alloys, which show that Stellite 6 has superior wear resistance to the others.

A series of Stellite alloys were tested under pin-on-disc sliding wear by Yao et al. [65]. The results are illustrated in Figure 2-17, which show that the wear resistance of Stellite alloy hardfacings varied and Stellite 728 was the most wear-resistant.

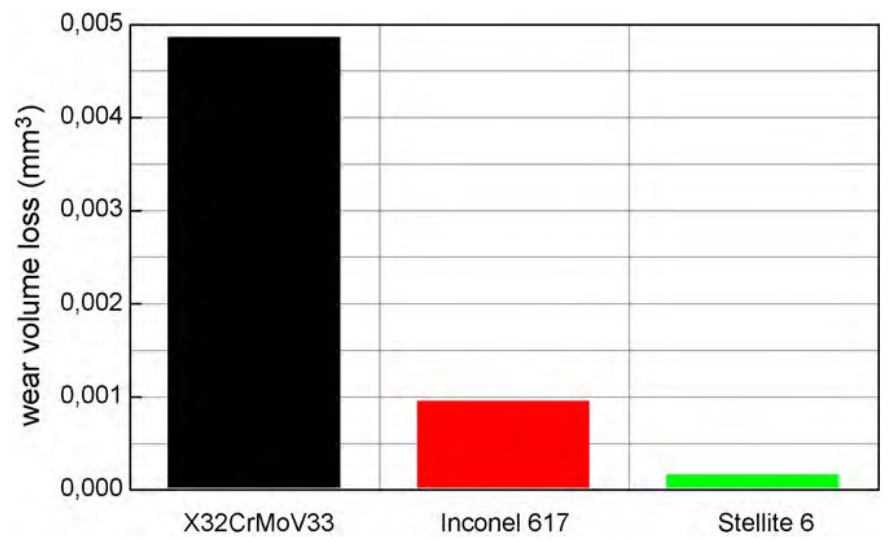

Figure 2-16: Wear volume losses of different alloys under high-temperature ball-on-disc sliding wear [64]. 


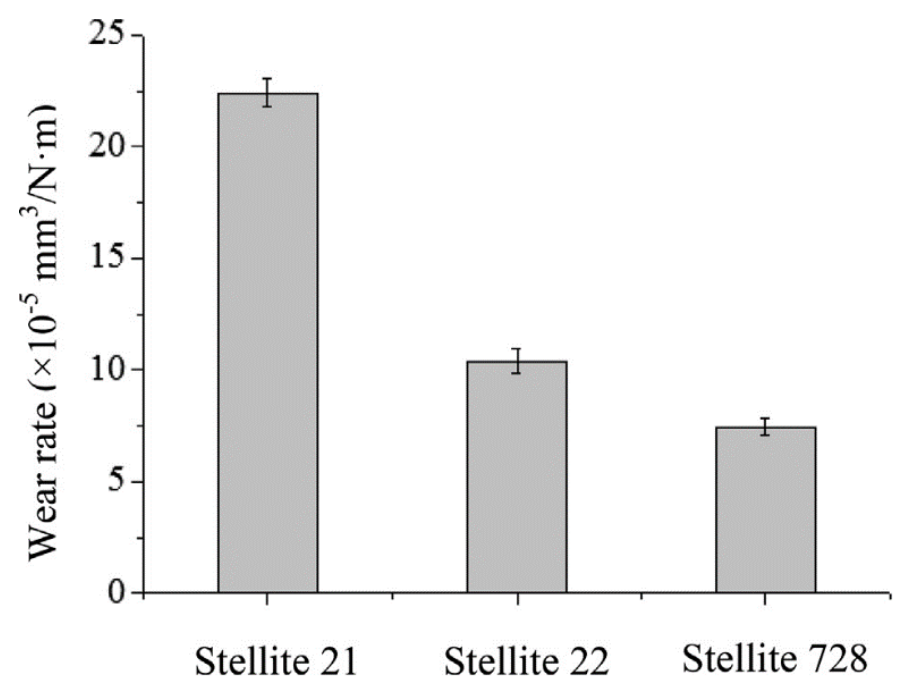

Figure 2-17: Comparison of wear rate between Stellite alloy hardfacings [65].

Kapoor et al. [4] investigated the wear behavior of Stellite alloys at room temperature and at elevated temperatures, with emphasis on the relationship between microstructure and wear resistance. The content of carbon plays a critical role to determine the wear resistance of Stellite alloys at room temperature, while the oxidation of the alloys dominantly contributed to the wear loss at high temperature. In this case, the amount of chromium as well as the carbon content are the key factors.

Collier et al. [23] studied the tribological performance of three molybdenum (Mo) Stellite alloys with varying carbon (C) and Mo contents using pin-on-disc dry-sliding wear test. The increasing contents of Mo and C contributed to the formation of carbides and intermetallics which can enhance the hardness and wear resistance of the alloys. Stellite 720 possesses more hard phases than other two alloys attributed to more $\mathrm{C}$ and Mo contents so that it had the best performance in this wear test compared to other Stellite alloys. 


\subsubsection{Corrosion test}

\subsubsection{Corrosion of materials}

Corrosion is a natural process that converts a refined metal into a more chemically-stable form such as oxide, hydroxide, or sulfide. It is a gradual destruction of materials (usually metals) by chemical or electrochemical reactions with working environment [66]. A common type of corrosion is rust, which is found on iron and steel structures. In this type of corrosion, the iron is reacting with oxygen to form iron oxide compounds.

Methods for studying the corrosion behavior of materials can be divided into three categories: electrochemical methods, immersion test, and microstructure analysis. Electrochemical methods are a series of analytical chemistry techniques used to measure the potential or current of an electrochemical cell. The immersion test method is a traditional means of studying the corrosion behavior of materials. Corrosive medium, $\mathrm{pH}$ value, temperature, exposure time, and pressure can be controlled as selective variables to simulate or accelerate the actual working conditions [67]. SEM, EDX, XRD and X-ray photoelectron spectroscopy (XPS) are the main methods for optical analyses of the microstructures of corroded surfaces and products.

\subsubsection{Electrochemical methods}

Electrochemical methods are well-known and convenient approaches to examining the corrosive performance of materials by controlling the voltage, time and concentration of the corrosive solution [68]. The main advantages of electrochemical methods are high sensitivity, accuracy, extensive linear dynamic range, and relatively low testing cost [69]. Outstanding progress has been made in electrochemical analysis since it was discovered. Monitoring electron flow is a measurement process, and it is the basis of electrochemical testing, because corrosion is 
an electrochemical reaction, and the current between the anode and cathode regions determines the corrosion rate [70]. Common electrochemical methods include potentiodynamic polarization, polarization resistance, cyclic polarization, and electrochemical impedance spectroscopy (EIS), etc.[71].

Potentiodynamic polarization method is representative of DC polarization technology. It has a wide range of applications in obtaining the corrosion rate, pitting sensitivity, passivity, and cathode performance of electrochemical systems. It is well known that anode, cathode, ions and the conductive path between anode and cathode are the four main conditions of the electrochemical process. Working electrode (WE), counter electrode (CE) and the solution between them create an ionic conduction path, while a potentiostat provides an electrical path. In this configuration, potentiostat can control and direct the electrochemical process. When electrons flow through the electrical path from the anode to the cathode, the anode and cathode are generally considered as the negative and positive electrodes, respectively [72]. The potential behavior of metals can be investigated via this method easily, which is favored in many applications. A typical hypothetical polarization curve with relevant parameters is shown in Figure 2-18. In the anode region, the behavior that current density does not rise with the increase of the voltage implies the formation of protective film on the metal surface during the corrosion process, which is recognized as passivation of the metal in polarization process. 


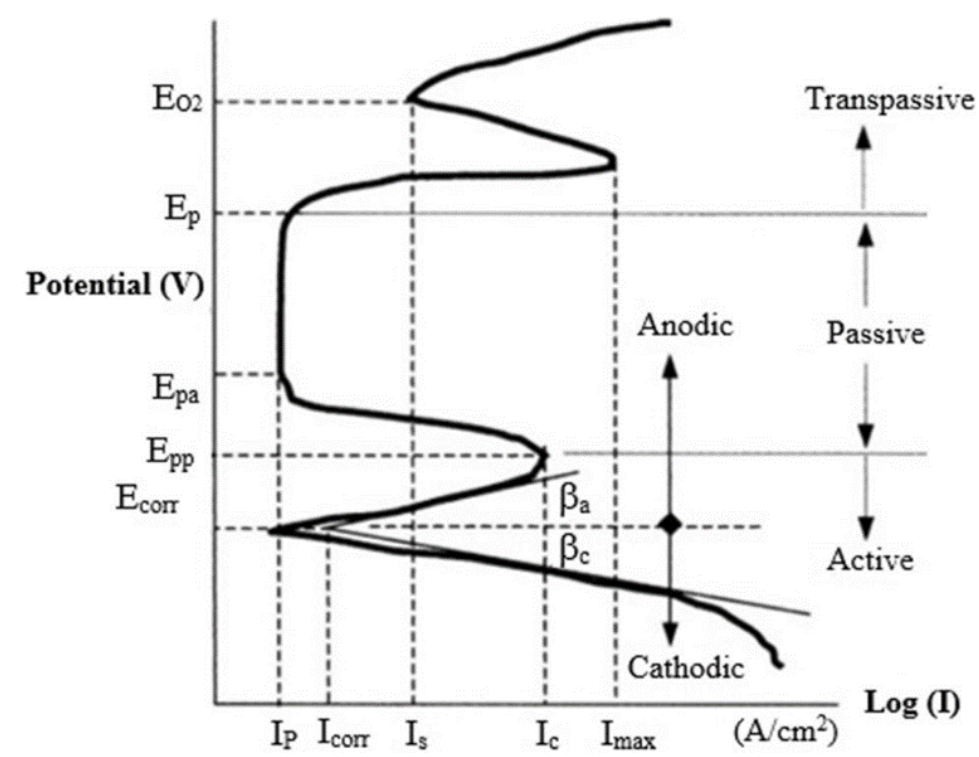

Figure 2-18: A typical hypothetical polarization curve with relevant parameters [73].

On this curve, there are two main parts: the cathode region and the anode region. The linear polarization relationship exists in the anode and cathode regions of the polarization curve (potential versus current density). From the turning point of the two areas, the important parameters that characterize the corrosion behavior of the tested material such as corrosion potential $\left(E_{c o r r}\right)$, current density $\left(I_{c o r r}\right)$, and polarization resistance $\left(R_{p}\right)$ can be determined by following Tafel extrapolation [67]. Corrosion current $\left(I_{\text {corr }}\right)$ can be obtained using Stern-Geary Equation [74]:

$$
I_{\text {corr }}=\frac{\beta_{a} \beta_{c}}{2.3039\left(\beta_{a}+\beta_{c}\right) R_{P}}
$$

The polarization resistance, $R_{p}$, can be determined from the following relationships [74]:

$$
\begin{gathered}
B=\frac{\beta_{a} \beta_{c}}{2.3039\left(\beta_{a}+\beta_{c}\right)} \\
R_{p}=\frac{B}{I_{\text {corr }}}
\end{gathered}
$$

Parameter $\beta_{a}$ and $\beta_{c}$ are called anode slope and cathode slope, respectively. $B$ represents Stern-Geary constant and can be calculated from the known Tafel slopes. Other parameters on this curve are given in Table 2-4. 
Table 2-4: Polarization relevant parameters [67].

\begin{tabular}{|c|c|}
\hline$I_{p}$ & Passive current density \\
\hline$I_{\text {corr }}$ & Corrosion current \\
\hline$I_{s}$ & Secondary current \\
\hline$I_{c}$ & Critical current density \\
\hline$I_{\text {max }}$ & Maximum current \\
\hline$E_{p a}$ & Passive potential \\
\hline$E_{c o r r}$ & Corrosion potential \\
\hline$E_{O_{2}}$ & Oxygen evolution \\
\hline$E_{p p}$ & Primary passive \\
\hline$E_{p}$ & Pitting potential \\
\hline
\end{tabular}

Electrochemical impedance spectroscopy (EIS) technique is also a common corrosion test method. Different from potentiodynamic testing, it is an AC polarization experimental technique to characterize an electrochemical system. This technology measures the impedance of the system over a frequency range corresponding to different interfaces in the electrolytic cell system. Using this method, a series of electrochemical parameters can be determined, including double ohmic resistance and double-layer capacitance, charge transfer resistance, etc.

If a potential is applied to the electrode system, the current will flow through the cell. The movement of ions through the electrolyte results in the formation of new materials. If the applied potential is a sine wave $(\Delta E \times \sin \omega t)$, the alternating current (AC) generated by the potential will also be a sine wave $(\Delta i \times \sin \omega t)$. The impedance is regarded as the relationship between the applied potential and the current. Moreover, this relationship is similar to the resistance-currentpotential relationship of a DC circuit. The impedance $(Z)$ has an amplitude $(\Delta E / \Delta i)$ and a phase $(\varphi)$. Therefore, EIS is the result of a series of impedances $(Z)$ with a series of different phases $(\varphi)$ [75].

The impedance $(Z)$ in an AC circuit behaves similarly to the resistance $(R)$ in a DC circuit. There are a number of instrument arrangements that can be used for EIS. One common instrument 
combination uses a potentiostat connected to a frequency response analyzer (FRA). During the test, some potential (often $E_{c o r r}$ ) is maintained by the potentiostat. At the same time, the FRA is employed to superimpose a small fluctuating potential and characterize the sample's response to this voltage stimulus [29].

The curves, named Nyquist plot, in Figure 2-19, represents the type of experimental data that would be generated for a bare metal experiencing activation polarization. In this case, the solution resistance $\left(R_{S}\right)$ is found using the high-frequency data, and the value for the charge transfer resistance $\left(R_{c t}\right)$ is defined by the low-frequency data. In the simplest case, the value of $R_{c t}$ is the same as that of the polarization resistance $\left(R_{p}\right)$. Once $R_{p}$ is defined, the corrosion rate is possibly obtained [29].

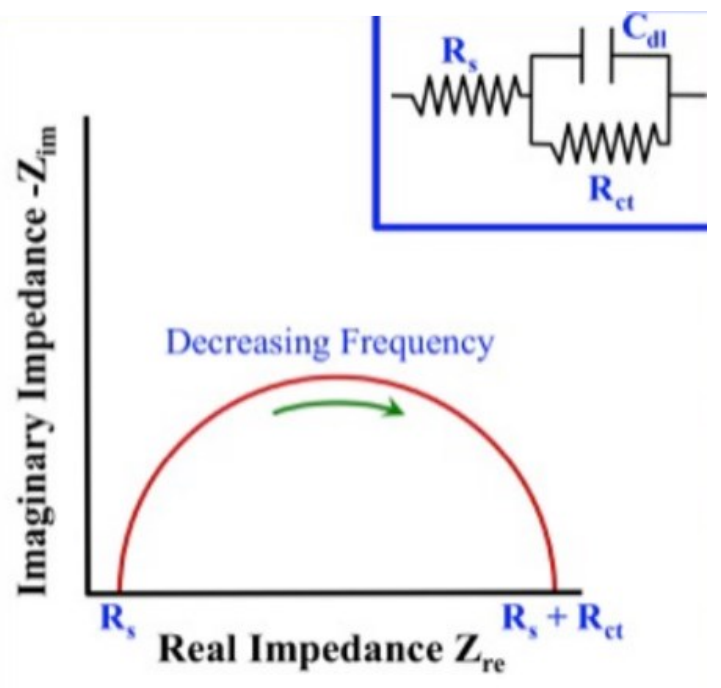

Figure 2-19: A typical diagram of electrochemical impedance spectroscopy (EIS) [29]. 


\subsubsection{Immersion method}

Immersion test is a traditional method of corrosion study. The objective of this testing is to simulate the actual working environment as much as possible or use highly corrosive media to accelerate the corrosion process. During the test, corrosion medium, $\mathrm{pH}$ value, temperature, exposure time, and pressure are selected as the control variables. The test results can be evaluated in various methods, such as mass change, volume change, pit depth, etc. [33], [67]. From the perspective of micro-morphology, SEM, XRD, EDX and XPS can also be employed to inspect and analyze the samples after immersion in medium.

\subsubsection{Related studies in corrosion test}

Electrochemical corrosion tests, including potentiodynamic and cyclic polarization tests, were conducted on four Stellite alloys in $3.5 \mathrm{wt} . \%$ sodium chloride $(\mathrm{NaCl})$ aqueous solution by Liu et al. [76]. The chemical compositions of these alloys are given in Table 2-5. Figure 2-20 shows the polarization curves that describe the response of the current to the applied potential on the electrochemical system. The calculated values of corrosion parameters from the polarization curves of the alloys are summarized in Table 2-6. Obviously, the high-Mo Stellite alloys, Stellite 22 and Stellite 728, have comparable corrosion resistance to Stellite 21, and higher corrosion resistance than Stellite 6. According to the values of $E_{c o r r}$, Stellite 22 was corroded easier than Stellite 728 and Stellite 21, but after corrosion started, it became severer on Stellite 728 due to the larger value of $I_{\text {corr }}$. The values of $R_{p}$ confirm that Stellite 728 was worse than Stellite 22 and Stellite 21 in corrosion resistance. 
Table 2-5: Chemical compositions (wt.\%, Co in balance) of Stellite alloys [76].

\begin{tabular}{|c|c|c|c|c|c|c|c|c|c|}
\hline Alloy & $\mathrm{Cr}$ & $\mathrm{W}$ & $\mathrm{Mo}$ & $\mathrm{C}$ & $\mathrm{Fe}$ & $\mathrm{Ni}$ & $\mathrm{Si}$ & $\mathrm{Mn}$ & $\mathrm{Nb}$ \\
\hline Stellite 22 & 27 & & 11 & 0.25 & 3 & 2.75 & 1 & 1 & \\
\hline Stellite 728 & 24.2 & & 11.8 & 0.35 & 1 & 3.8 & 0.45 & 0.52 & 2.07 \\
\hline Stellite 21 & 27 & & 5.5 & 0.25 & 3 & 2.75 & 1 & 1 & \\
\hline Stellite 6 & 28.5 & 4.5 & 1.5 & 1.2 & 3 & 3 & 1.5 & 1 & \\
\hline
\end{tabular}

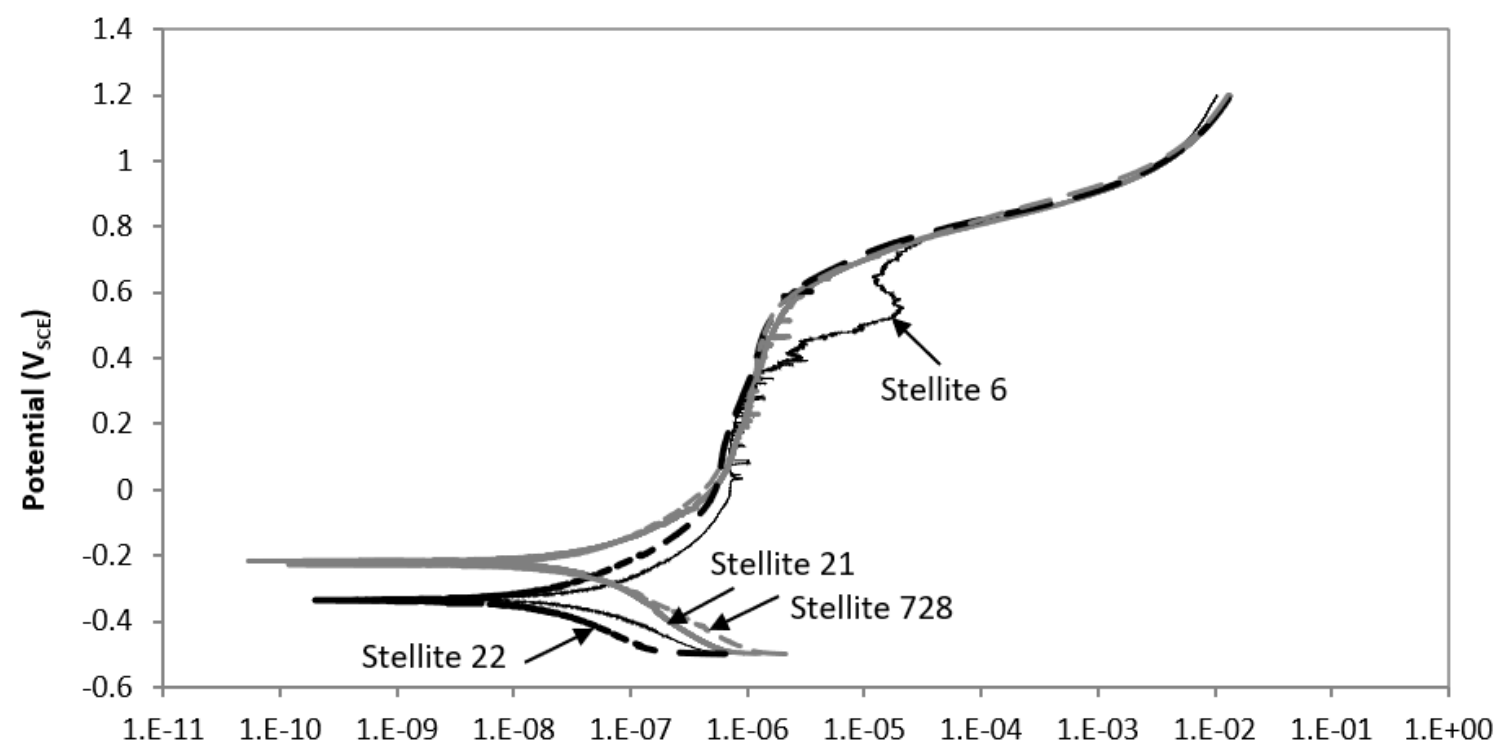

Figure 2-20: Potentiodynamic polarization curves of Stellite alloys in $3.5 \mathrm{wt} . \% \mathrm{NaCl}$ solution [76].

Table 2-6: Summary of polarization test results of Stellite alloys in $3.5 \mathrm{wt.} \% \mathrm{NaCl}$ solution [76].

\begin{tabular}{cccc}
\hline Alloy & $E_{\text {corr }}\left(\mathrm{V}_{\mathrm{SCE}}\right)$ & $I_{\text {corr }}\left(\mu \mathrm{A} / \mathrm{cm}^{2}\right)$ & $R_{p}\left(\mathrm{k} \Omega \mathrm{cm}^{2}\right)$ \\
\hline Stellite 22 & -0.325 & 0.004 & $3.418 \times 10^{5}$ \\
Stellite 728 & -0.215 & 0.009 & $1.408 \times 10^{5}$ \\
Stellite 21 & -0.210 & 0.004 & $3.398 \times 10^{5}$ \\
Stellite 6 & -0.320 & 0.014 & $8.978 \times 10^{4}$ \\
\hline
\end{tabular}

Zhang et al. [67] investigated the corrosion behavior of wrought Stellite 6K and Stellite 6B, which have different carbon contents, in $3.5 \mathrm{wt} . \% \mathrm{NaCl}$ solution and Green Death solution. The electrochemical test results indicated that Stellite 6K was easier to get corroded than Stellite 6B due to its larger volume fraction of carbides, but Stellite $6 \mathrm{~K}$ exhibited better oxide film $\left(\mathrm{Cr}_{2} \mathrm{O}_{3}\right)$ 
behavior. The immersion tests of Stellite $6 \mathrm{~K}$ and Stellite $6 \mathrm{~B}$ in Green Death solution showed that Stellite 6B had better resistance to pitting corrosion.

Kamal et al. [77] evaluated the corrosion performance of high-carbon high-molybdenum Stellite alloys including Stellite 706, Stellite 712 and Stellite 720 in various corrosive media under electrochemical tests . The results revealed excellent corrosion resistance of these alloys in Green Death solution, $3.5 \% \mathrm{NaCl}$ solution and morpholine solution with $\mathrm{pH}$ 9.5. From the analyses of the corroded surface with SEM/EDX, the excellent corrosion resistance of Stellite 700 series was suggested to be attributed to the formation of passivation film which benefited from additional Mo content. Both carbon and molybdenum contents affected the passivation film and high content of Mo stabilized the passivation film. 


\section{Chapter 3: Alloy Powder Characterization and Bulk Alloy Microstructural}

\section{Analysis}

\subsection{Raw Powder Specification and Examination}

\subsubsection{Chemical composition}

A benchmark Stellite grade, Stellite 6, and a newly designed cobalt high entropy alloy (HEA), HE6, were studied in this research. The nominal chemical compositions of the alloy powders (wt.\% and at.\%) are given in Table 3-1. Based on the composition, the values of entropy $(\Delta \mathrm{S})$ and other critical parameters in the HEA regime can be calculated for Stellite 6 and HE6 alloy using Equation 2.2, 2.7 to 2.11 in Chapter 2, and they are reported in Table 3-2. The value of $\delta$ is related to the atomic size difference calculated with the atomic composition and the atomic radius among constituent elements. The parameter $\Omega$ reflects whether entropy or enthalpy effect is predominant in the alloy system. The value $\Omega>1$ is preferred for HEAs. The parameter $V E C$ is defined as the weighted sum of electrons accommodated in the valence band, which can be used for predicting the phase stability of the FCC and BCC portion in HEAs. According to VEC categorization, HE6 possibly has FCC and BCC mixed solid solution.

Table 3-1: Chemical compositions of alloy powders.

\begin{tabular}{|c|c|c|c|c|c|c|c|c|}
\hline \multicolumn{2}{|c|}{ Alloy } & \multicolumn{7}{|c|}{ Element } \\
\cline { 3 - 9 } Stellite 6 & wt.\% & 55.3 & 29 & 4.5 & 1.5 & 3 & 3 & 1.20 \\
\cline { 2 - 9 } & at.\% & 51.8 & 30 & 1.4 & 0.9 & 3 & 3 & 5.52 \\
\hline \multirow{2}{*}{ HE6 } & wt.\% & 20.1 & 18 & 19.0 & 3.0 & 19 & 20 & 0.96 \\
\cline { 2 - 9 } & at.\% & 21.6 & 22 & 6.5 & 2.0 & 22 & 22 & 5.06 \\
\hline
\end{tabular}


Table 3-2: Entropy and related parameters of selected alloys.

\begin{tabular}{|c|c|c|c|c|c|}
\hline Alloy & Entropy $(\Delta \mathbf{S})$ & Enthalpy $(\Delta \mathbf{H}) \mathbf{( K J} / \mathbf{m o l})$ & $\begin{array}{c}\boldsymbol{\delta} \\
(\mathbf{\%})\end{array}$ & $\mathbf{\Omega}$ & VEC \\
\hline Stellite 6 & $1.32 \mathrm{R}$ & -17.229 & 3.73 & 1.05 & 7.56 \\
\hline HE6 & $1.73 \mathrm{R}$ & -11.892 & 3.83 & 2.55 & 7.84 \\
\hline
\end{tabular}

In terms of Co content, HE6 alloy only has about one-third of the amount compared to Stellite 6 in weight percentage, which has significantly reduced the use of Co which is the iconic element in the traditional Stellite alloy system. Stellite 6 also contains much higher $\mathrm{Cr}$ (29wt.\%) than HE6 (18 wt.\%), but Stellite 6 has a little higher C (1.20 wt.\%) content than HE6 (0.96 wt.\%). On the other hand, W, Fe and Ni contents are much higher in HE6 than in Stellite 6, and the Mo content is doubled. According to the HEA criteria, there are six elements in HE6 that are larger than 5 at.\%, $\mathrm{Co}, \mathrm{Cr}, \mathrm{W}, \mathrm{Fe}, \mathrm{Ni}$ and $\mathrm{C}$, which are considered as major elements; one element, Mo, is less than 5 at.\%, which is the minor element. Therefore, a chemical formula according to at. $\%$ can be assigned to $\mathrm{HE} 6, \mathrm{CoCrW}_{0.3} \mathrm{Mo}_{0.09} \mathrm{FeNiC}_{0.23}$. The influence of the chemical changes from Stellite 6 to HE6 was studied via various experiments in this research and the results will be reported and discussed in the following chapter.

\subsubsection{Surface morphology}

In this study, Stellite 6 was selected as a conventional Stellite grade, and HE6 was created in terms of high entropy alloy regime. Both grades were manufactured from atomized alloy powders, which were supplied by Kennametal Stellite Inc. There is one sieve size $(45 \mu \mathrm{m})$ of powder for Stellite 6 , and two sizes $(45 \mu \mathrm{m}$ and $150 \mu \mathrm{m})$ for HE6.

Morphology analysis was conducted on these alloy powders before the manufacturing processes of making bulk materials. The powder samples were examined using SEM with a 
back-scatter electron (BSE) imaging setting (Figure 3-1). The electrons on the sample were stimulated by the incident beams from the electron gun and scattered. BSE detectors above the sample collected electrons scattered as a display of sample composition, whereas detectors placed to the side collected electrons scattered as a function of surface topography. Due to the difference in phase composition, the SEM image taken by BSE can better display the surface morphology of the sample.

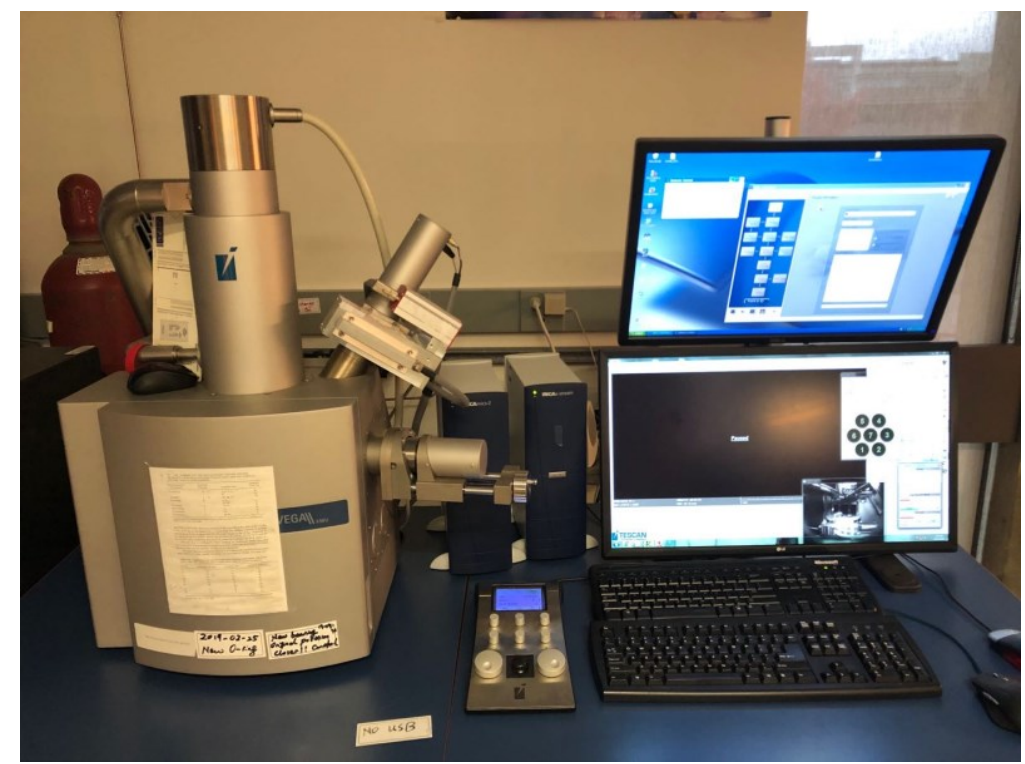

Figure 3-1: Tescan scanning electron microscope (SEM) system.

The surface topographies of the alloy powders are shown in Figure 3-2 to Figure 3-4. Dendritic microstructure on the spherical surfaces are observed obviously for all powders, which indicates that the alloying elements in the powders were fully interacted and diffused during the powder processing, i.e., typical casting microstructures had already been achieved in the powder stage. 


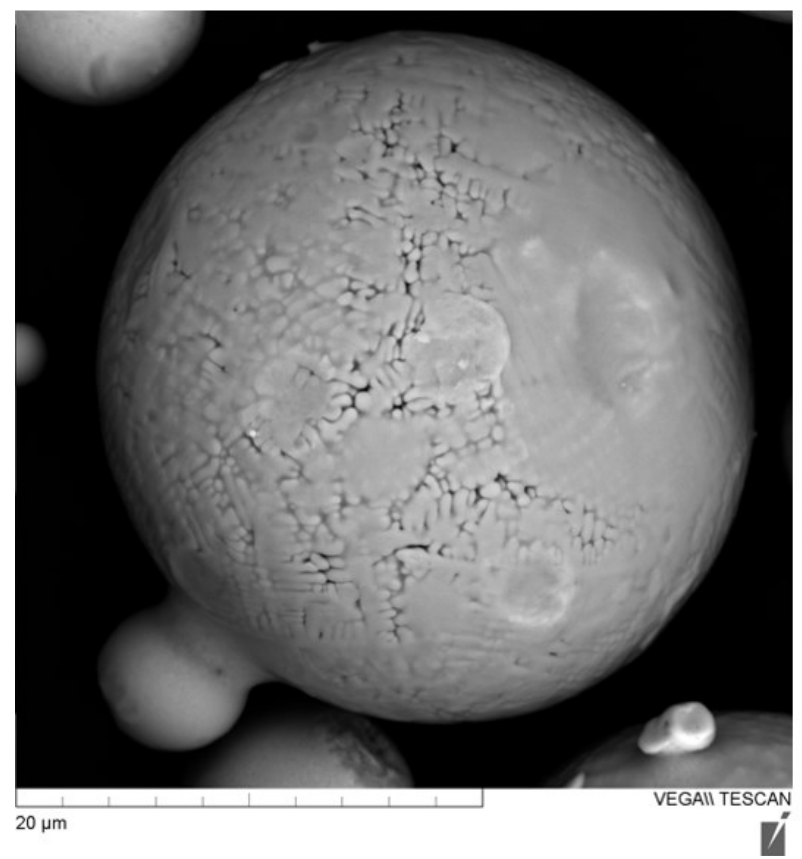

(a)

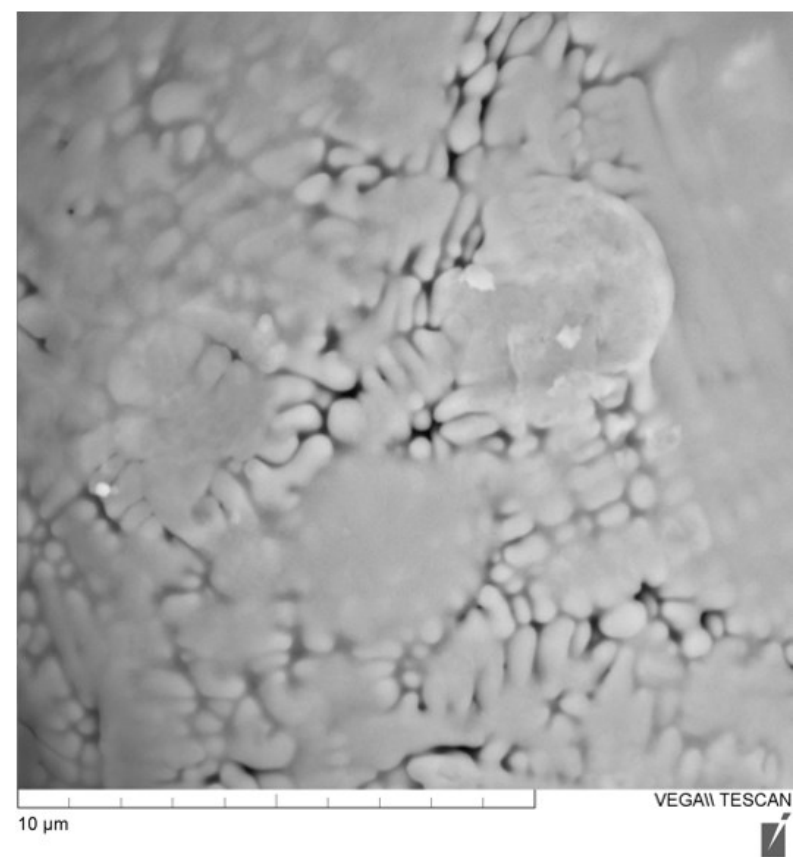

(b)

Figure 3-2: SEM microstructure of Stellite 6 powder with $45 \mu \mathrm{m}$ (a) low magnification, (b) high magnification.

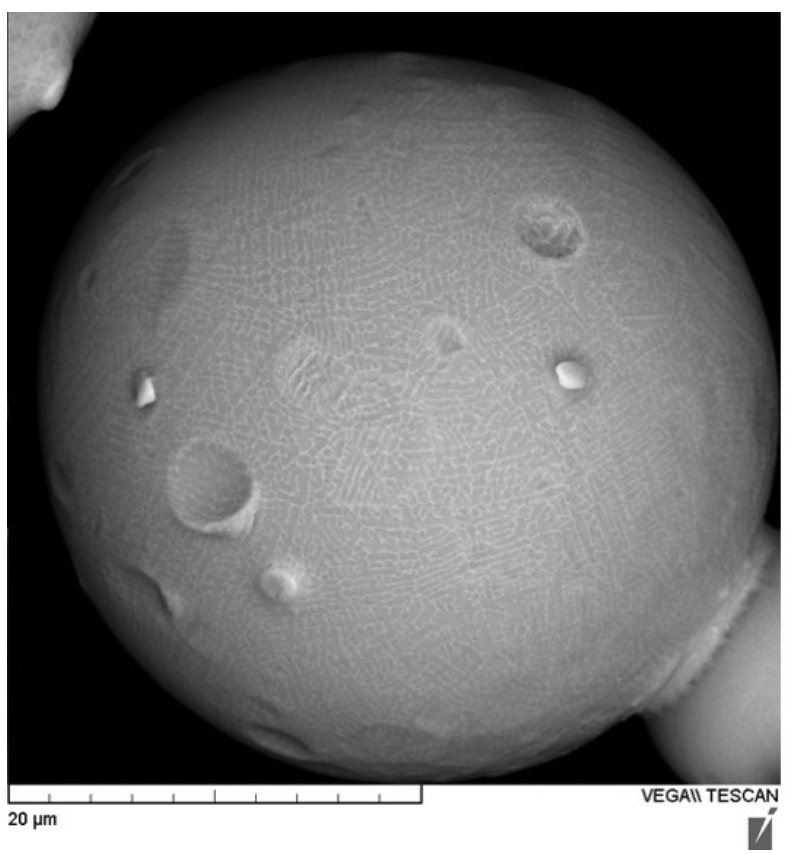

(a)

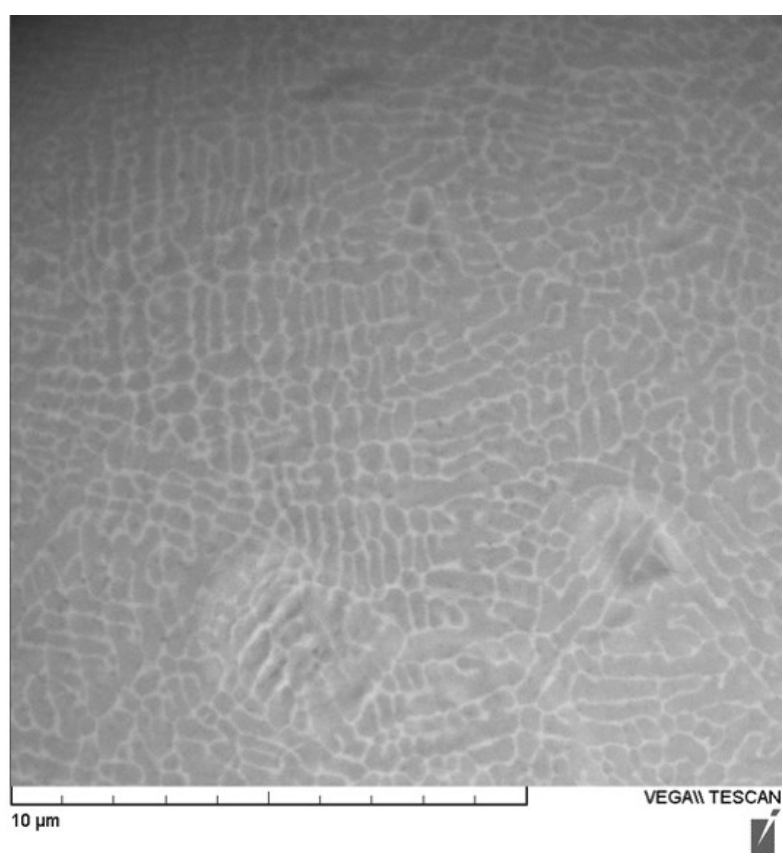

(b)

Figure 3-3: SEM microstructure of HE6 powder with $45 \mu \mathrm{m}$ (a) low magnification, (b) high magnification. 


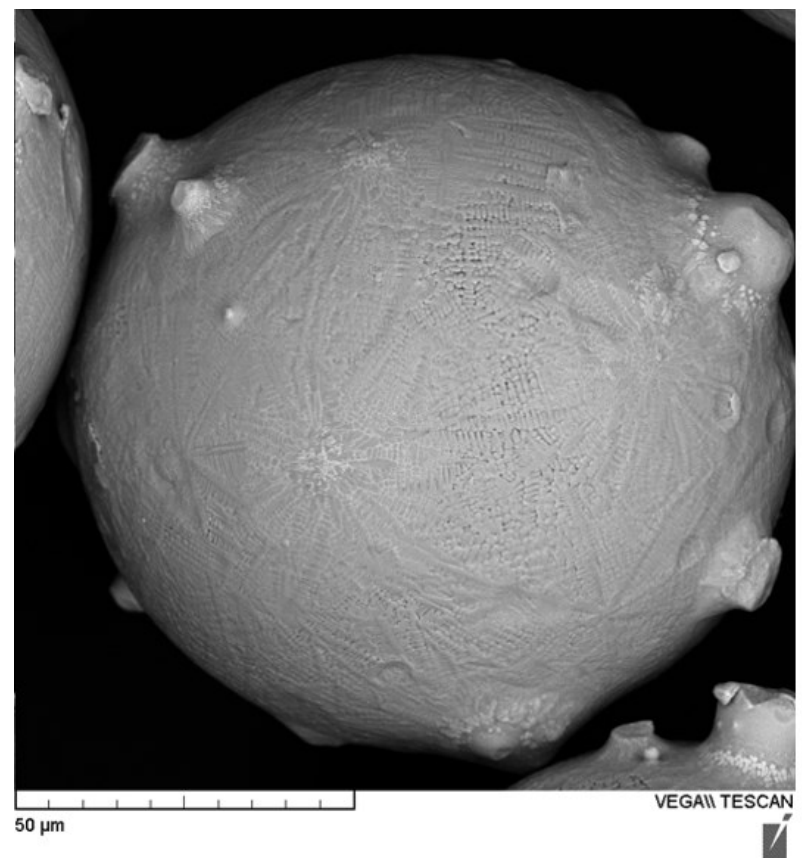

(a)

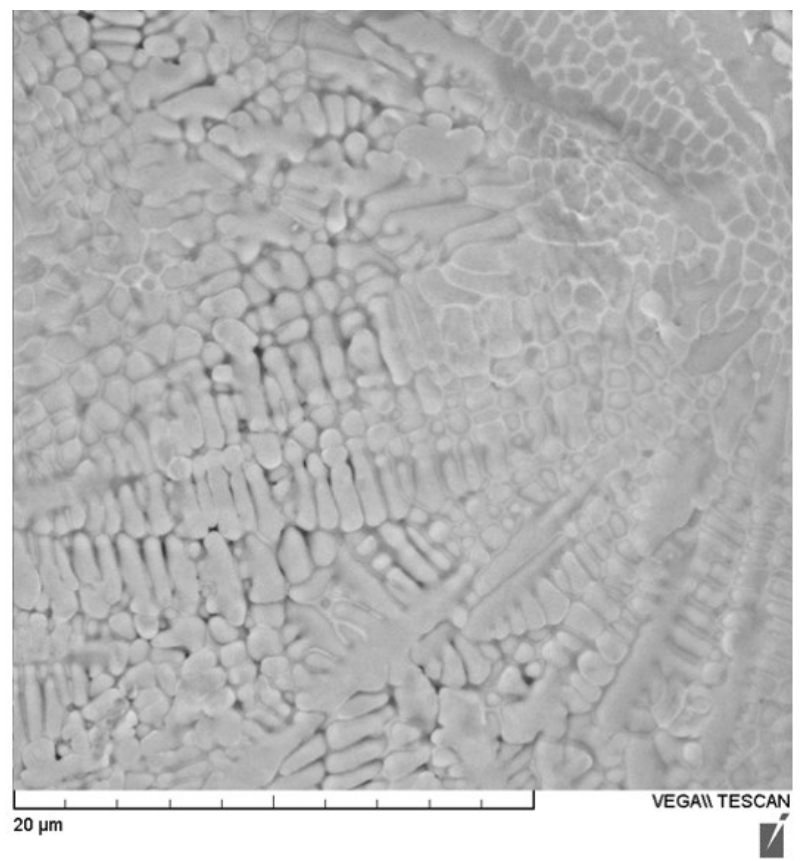

(b)

Figure 3-4: SEM microstructure of HE6 powder with $150 \mu \mathrm{m}$ (a) low magnification, (b) high magnification.

\subsubsection{Powder size distribution}

Based on the SEM images of the powders, the size distribution of each type of the powders can be analyzed through Image J processing software (Version 5.3.2). Figure 3-5 shows an instance of the threshold result based on the color difference displayed in the SEM images. 


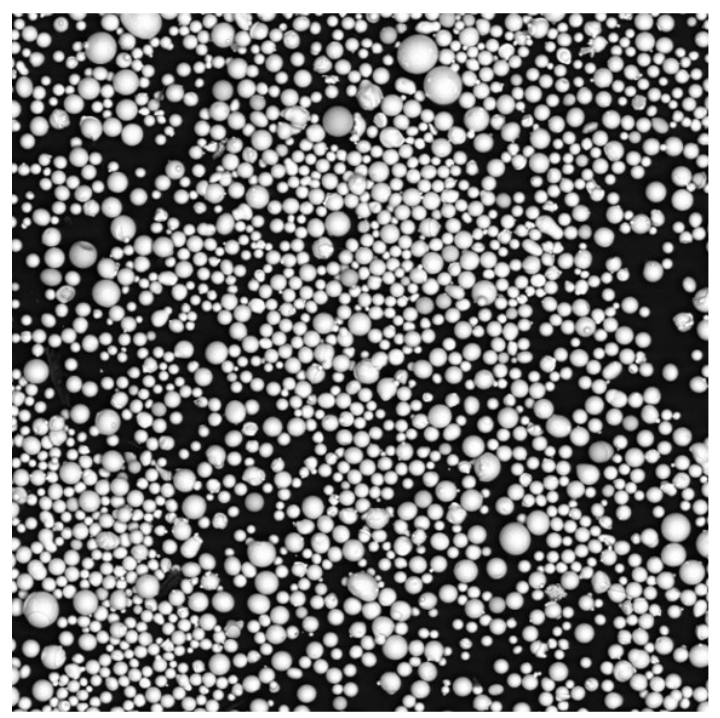

(a)

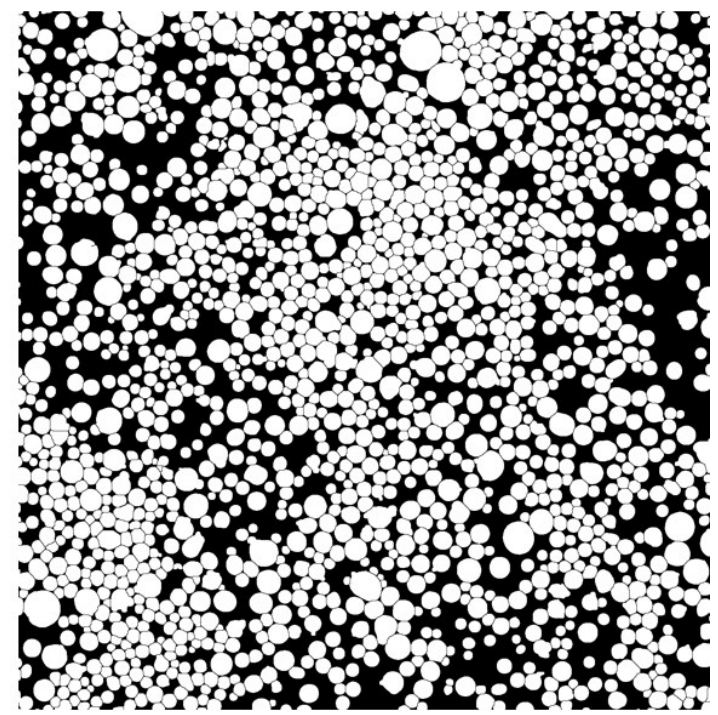

(b)

Figure 3-5: An ImageJ processing result of powder size distribution (a) original SEM image of HE6 $(45 \mu \mathrm{m})$, (b) software processed image.

The size distributions of three powders are presented in Figure 3-6 to Figure 3-8, and the statistic $d_{90}$ values are also reported. From the histograms, all powders show a statistic normal distribution. For Stellite $6, \mathrm{~d}_{90}$ is $35.04 \mu \mathrm{m}$, which means that $90 \%$ particles of Stellite 6 powder in this batch have diameters smaller than it. For HE6 $(45 \mu \mathrm{m})$ powder, the value of $\mathrm{d}_{90}$ is $39.47 \mu \mathrm{m}$, which reflects a similar distribution to Stellite $6(45 \mu \mathrm{m})$ powder. For HE6 powder with a larger size, its $\mathrm{d}_{90}$ equals $86.68 \mu \mathrm{m}$. All these results verify the factory mash/sieve particle size information that was provided by the supplier. 


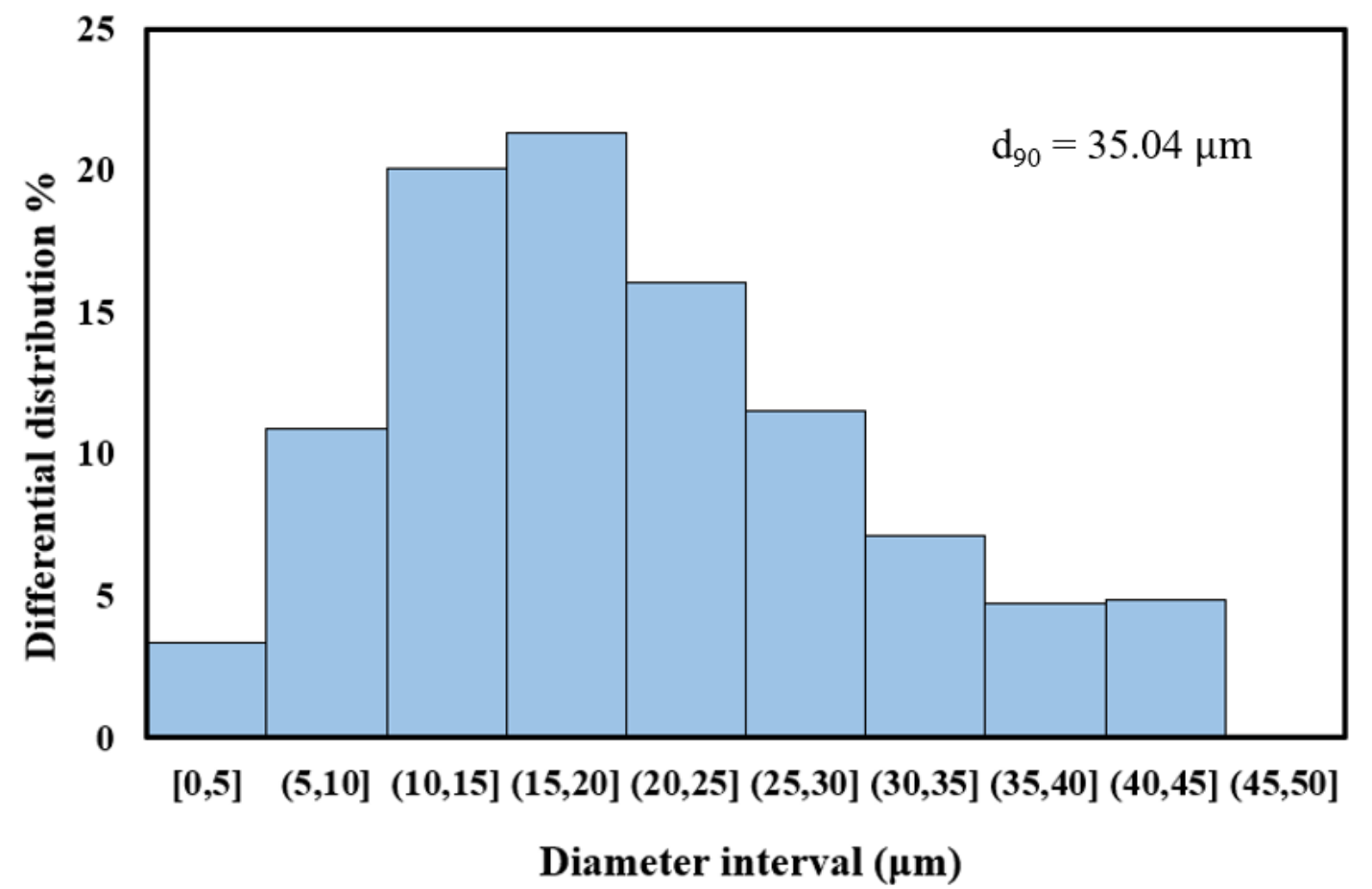

Figure 3-6: Particle size distribution of Stellite $6(45 \mu \mathrm{m})$ powder.

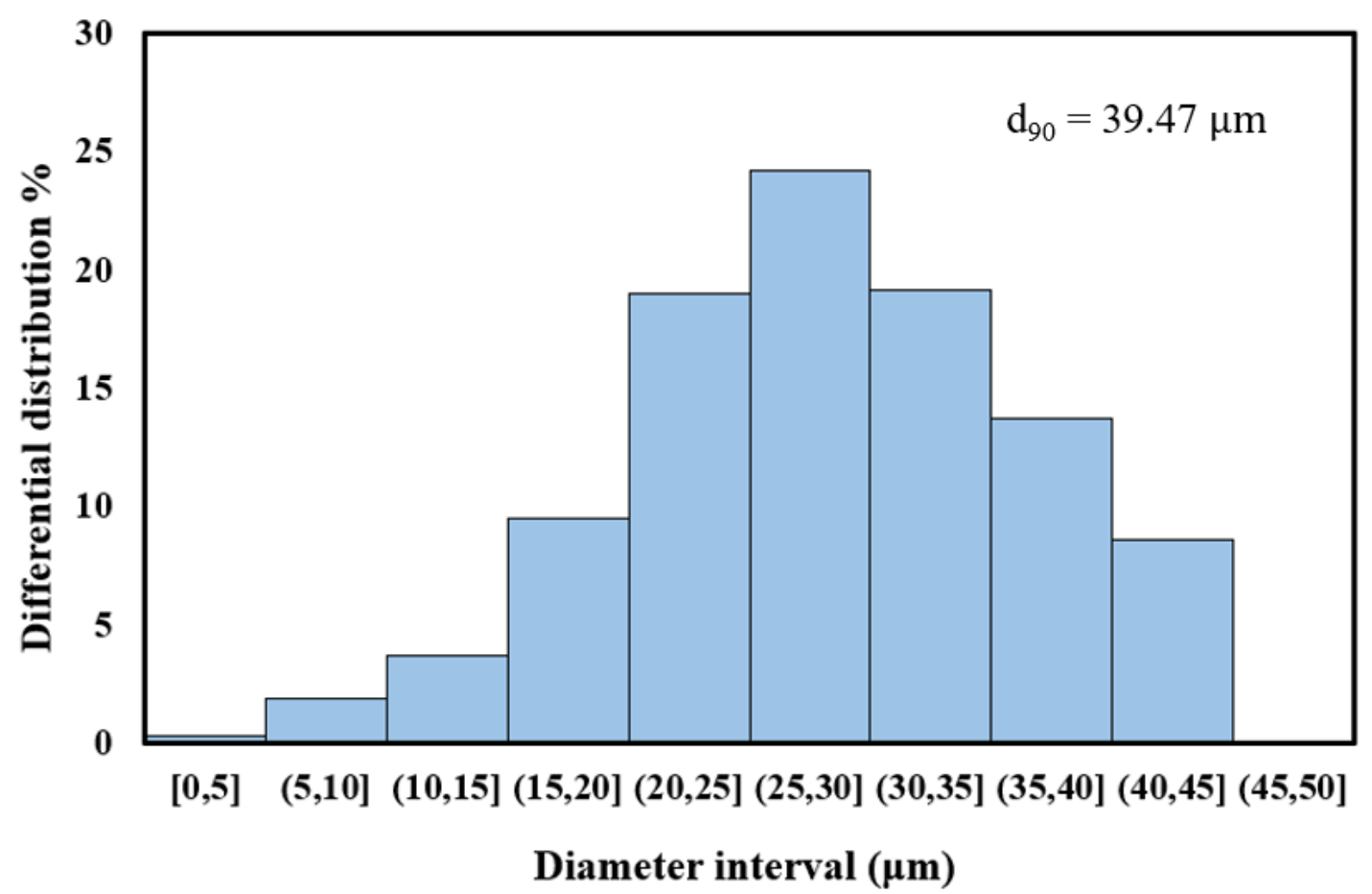

Figure 3-7: Particle size distribution of HE6 $(45 \mu \mathrm{m})$ powder. 


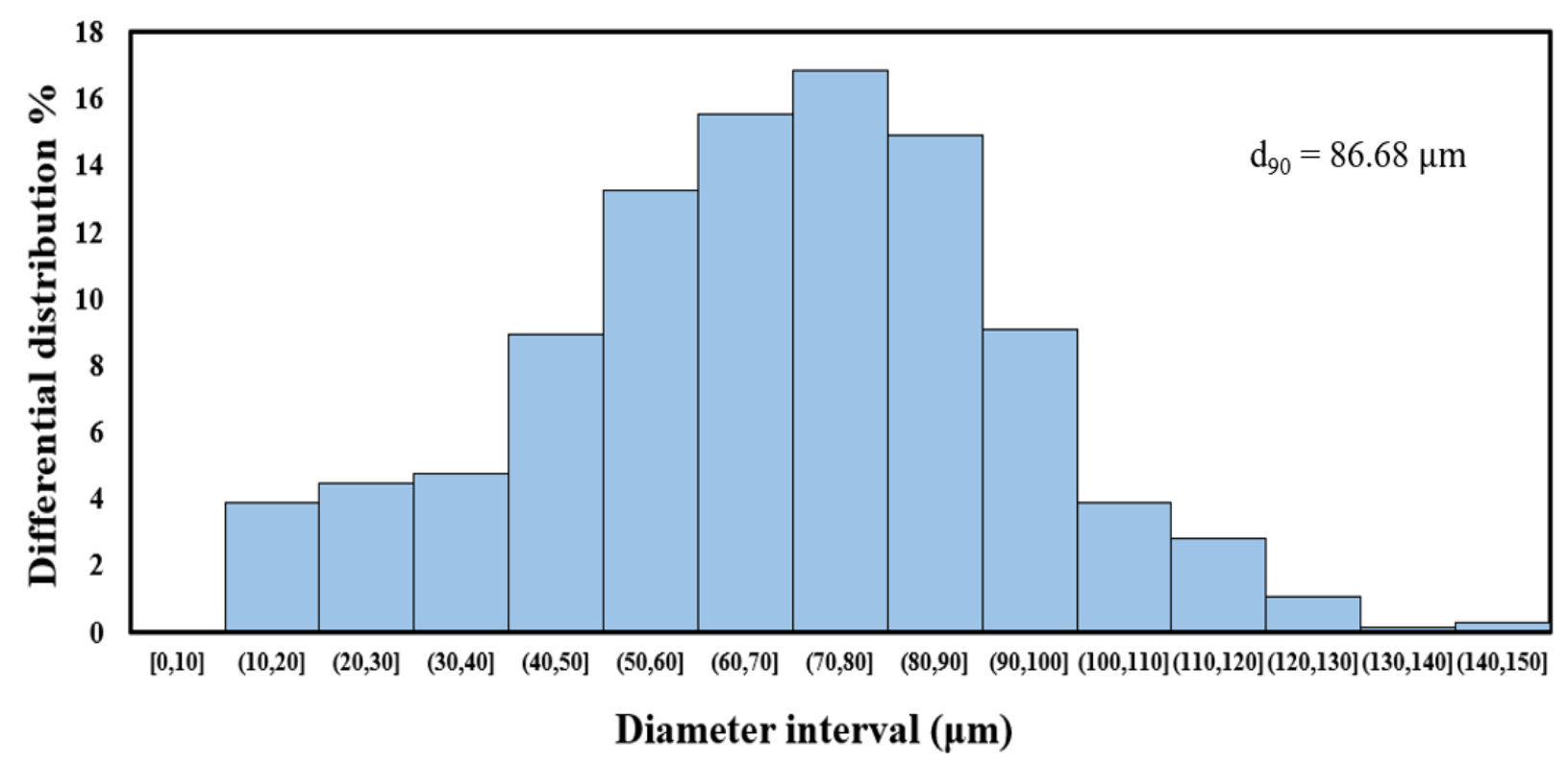

Figure 3-8: Particle size distribution of HE6 $(150 \mu \mathrm{m})$ powder.

\subsubsection{Differential scanning calorimetry (DSC) analysis}

Differential scanning calorimetry (DSC) test was conducted on the alloy powders with a Netzsch DSC 404 C instrument (Figure 3-9). DSC can detect calorimetric events that occur while material in the heating and cooling process, especially it can determine the heating point and solidification point of the material, which are the key features widely used in material categorization. A small amount of alloy powder weighing around $50 \mathrm{mg}$ was used for the DSC test. A vacuum coupled with a 5.0 grade Ar gas purifying system was equipped to keep the testing chamber free of impurities. The thermal cycle used in this study is shown in Figure 3-10. During the DSC test, the alloy powder sample was first heated to $500{ }^{\circ} \mathrm{C}$ at a heating rate of $5 \mathrm{~K} / \mathrm{min}$ and kept for 10 min to stabilize the crucible; then the sample was heated to the maximum temperature $\left(1450^{\circ} \mathrm{C}\right)$, at a rate of $10 \mathrm{~K} / \mathrm{min}$ and held for $10 \mathrm{~min}$; afterwards, the sample was cooled down to room temperature at a rate of $10 \mathrm{~K} / \mathrm{min}$. The details in mass record of each test are summarized in Table 3-3. The calorimetry behavior of the material during the heating and cooling process was 
recorded by the NETZSCH Measurement device (Version 4.2) and analyzed with the NETZSCH Thermal Analysis (Version 4.2) software. Two repeated tests were conducted on each powder to confirm the repeatability of the results.

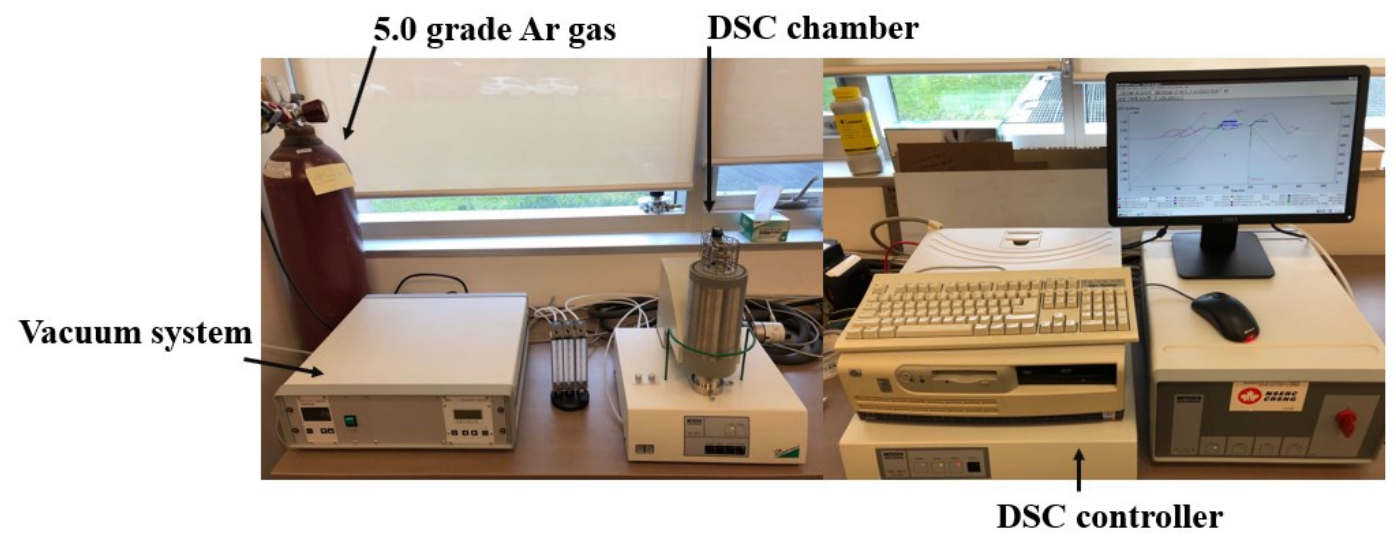

Figure 3-9: Netzsch-DSC $404 \mathrm{C}$ Thermal Analyzer.

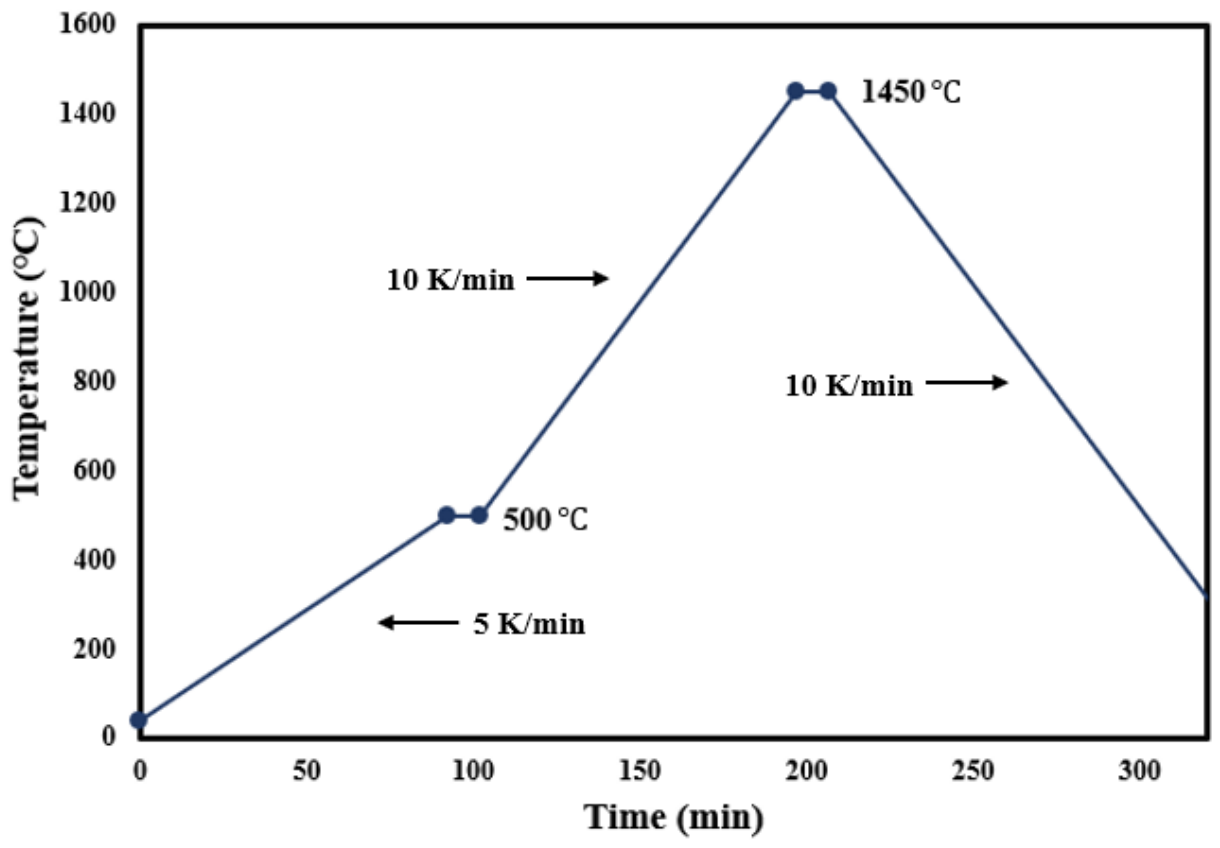

Figure 3-10: DSC thermal cycle. 
Table 3-3: Mass record sheet of DSC tests.

\begin{tabular}{|c|c|c|c|}
\hline Test group & $\begin{array}{c}\text { Weight of crucible } \\
(\mathrm{mg})\end{array}$ & $\begin{array}{c}\text { Weight of powder } \\
\text { (mg) }\end{array}$ & $\begin{array}{c}\text { Reference crucible } \\
(\mathrm{mg})\end{array}$ \\
\hline \multirow{2}{*}{ Stellite 6} & 315.4 & 49.9 & \multirow{6}{*}{312.2} \\
\hline & 330.1 & 50.4 & \\
\hline \multirow{2}{*}{ HE6 (45 $\mu \mathrm{m})$} & 315.0 & 50.1 & \\
\hline & 323.5 & 50.1 & \\
\hline \multirow{2}{*}{ HE6 $(150 \mu \mathrm{m})$} & 320.5 & 50.1 & \\
\hline & 314.1 & 50.3 & \\
\hline
\end{tabular}

The DSC curves obtained from the melting (heating) and solidification (cooling) process for the three powders are presented in Figure 3-11 to Figure 3-13. When a substance is crystallized, since the free energy of a regular crystal lattice is less than the free energy of a disordered liquid state, an exothermic effect occurs (valleys). Conversely, the melting of the crystal causes an endothermic effect (peak). Therefore, solidification temperature is the temperature at which the last exothermic peak occurs in the cooling process, while the melting point is the temperature at the first endothermic peak in the heating process [78]. The melting and solidification points of the alloy powders determined from the DSC tests are summarized in Table 3-4.

For the two sizes of HE6 powder, the powder size has little effect on both heating and cooling behavior since the two DSC curves are almost identical (Figure 3-14 and Figure 3-15). Between the two different alloy grades, the melting point of Stellite 6 is about $26^{\circ} \mathrm{C}$ lower than that of HE6; the solidification temperature of Stellite 6 is also lower than that of HE6.

Table 3-4: Melting and solidification point data.

\begin{tabular}{|c|c|c|}
\hline Powder & Melting point $\left({ }^{\circ} \mathrm{C}\right)$ & Solidification point $\left({ }^{\circ} \mathrm{C}\right)$ \\
\hline Stellite $\mathbf{6}(\mathbf{4 5} \boldsymbol{\mu m})$ & $1260.4 \pm 0.2$ & $1112.9 \pm 1.3$ \\
\hline HE6 (45 $\boldsymbol{\mu m})$ & $1285.9 \pm 0.1$ & $1250.0 \pm 0.4$ \\
\hline HE6 $(\mathbf{1 5 0} \boldsymbol{~} \mathbf{m})$ & $1285.8 \pm 0.1$ & $1249.4 \pm 1.0$ \\
\hline
\end{tabular}




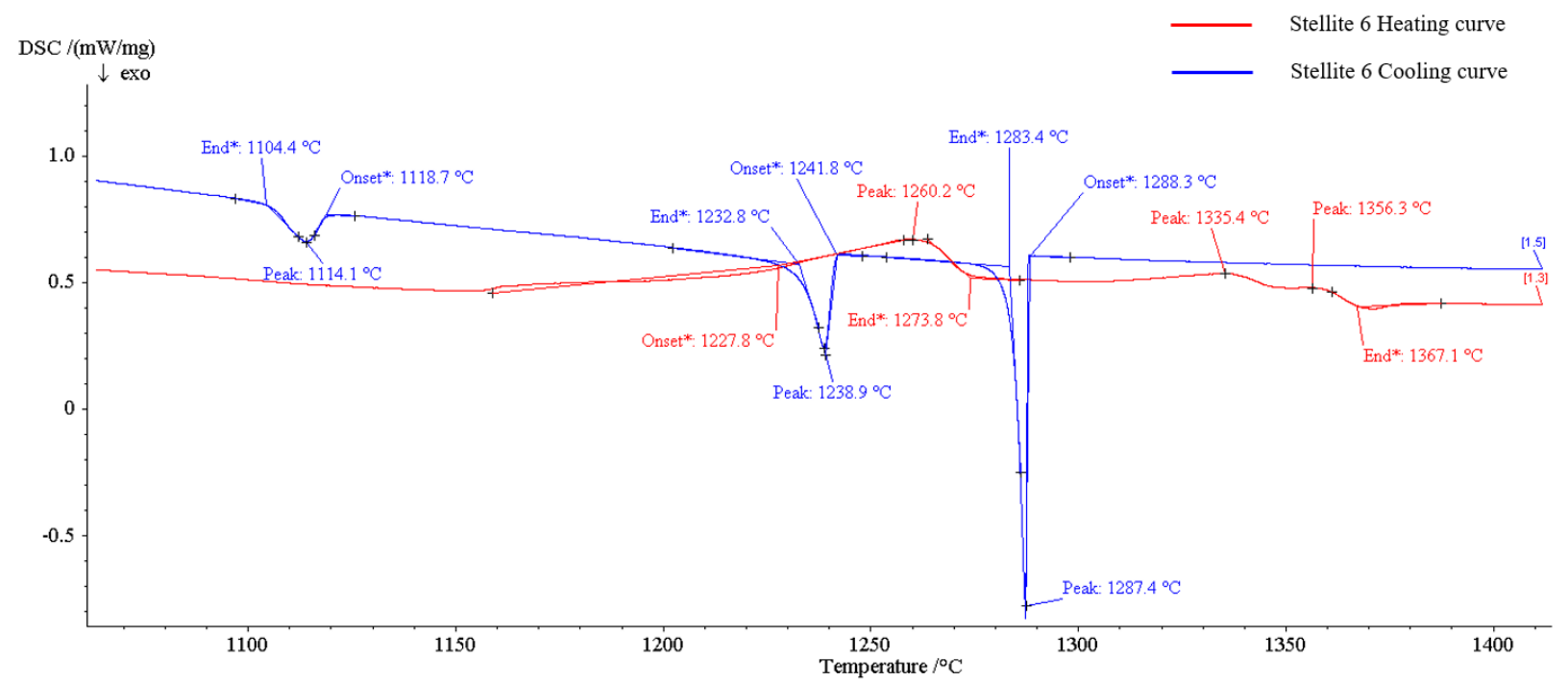

Figure 3-11: DSC curves of Stellite $6(45 \mu \mathrm{m})$ powder.

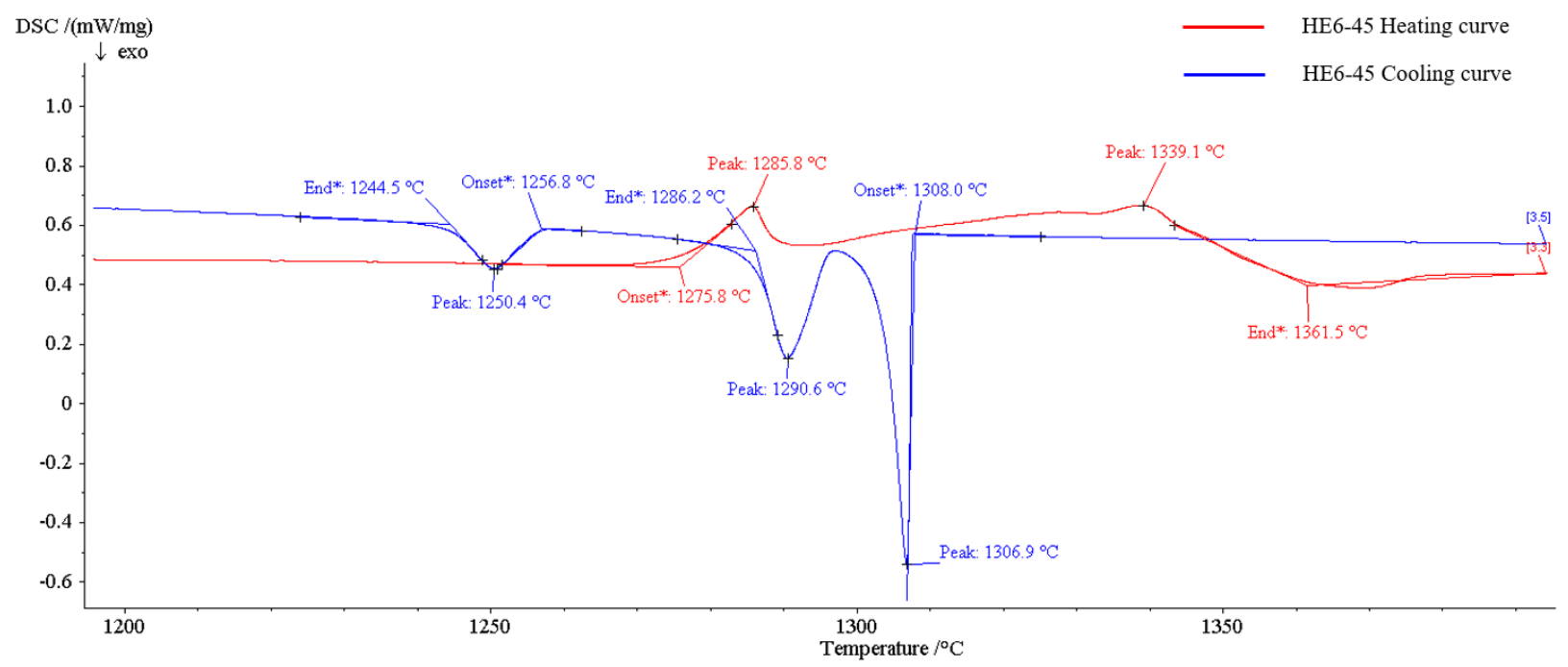

Figure 3-12: DSC curves of HE6 $(45 \mu \mathrm{m})$ powder. 


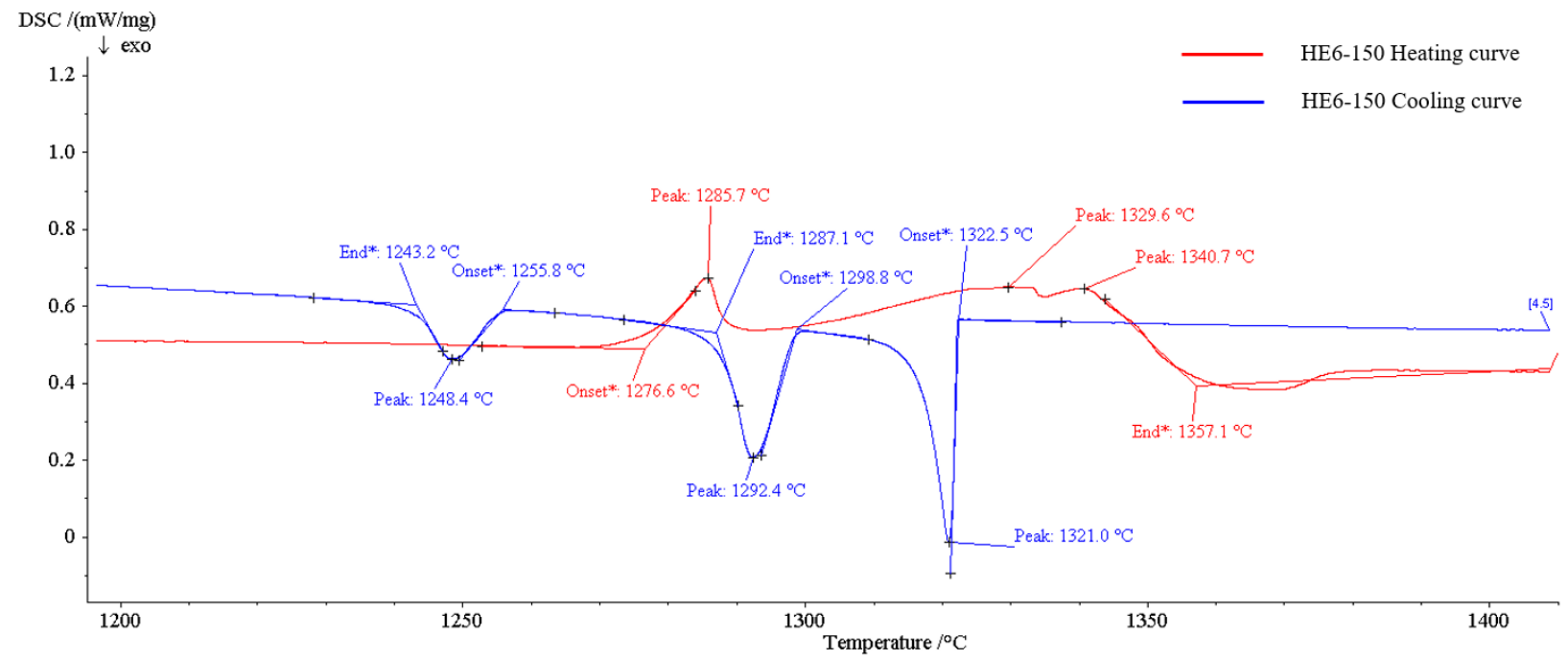

Figure 3-13: DSC curves of HE6 $(150 \mu \mathrm{m})$ powder.

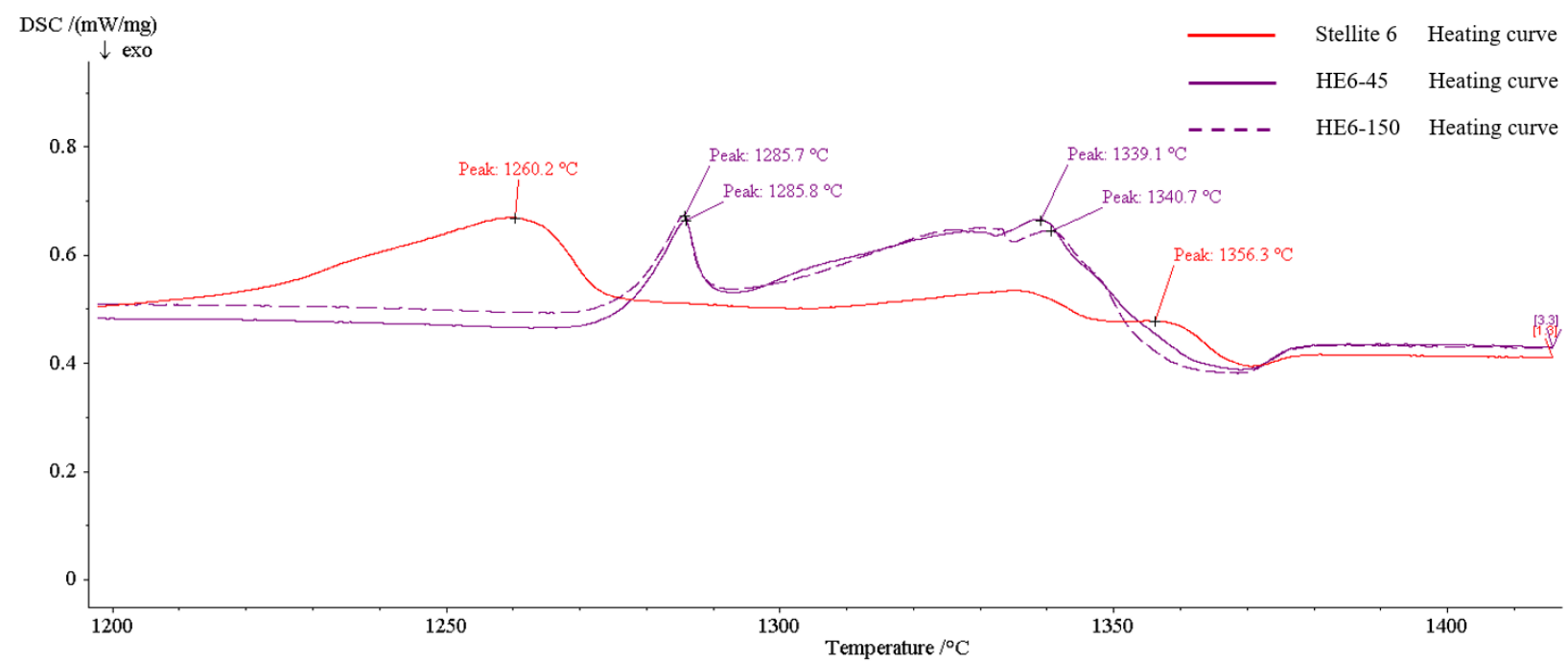

Figure 3-14: DSC heating curves of tested powders. 


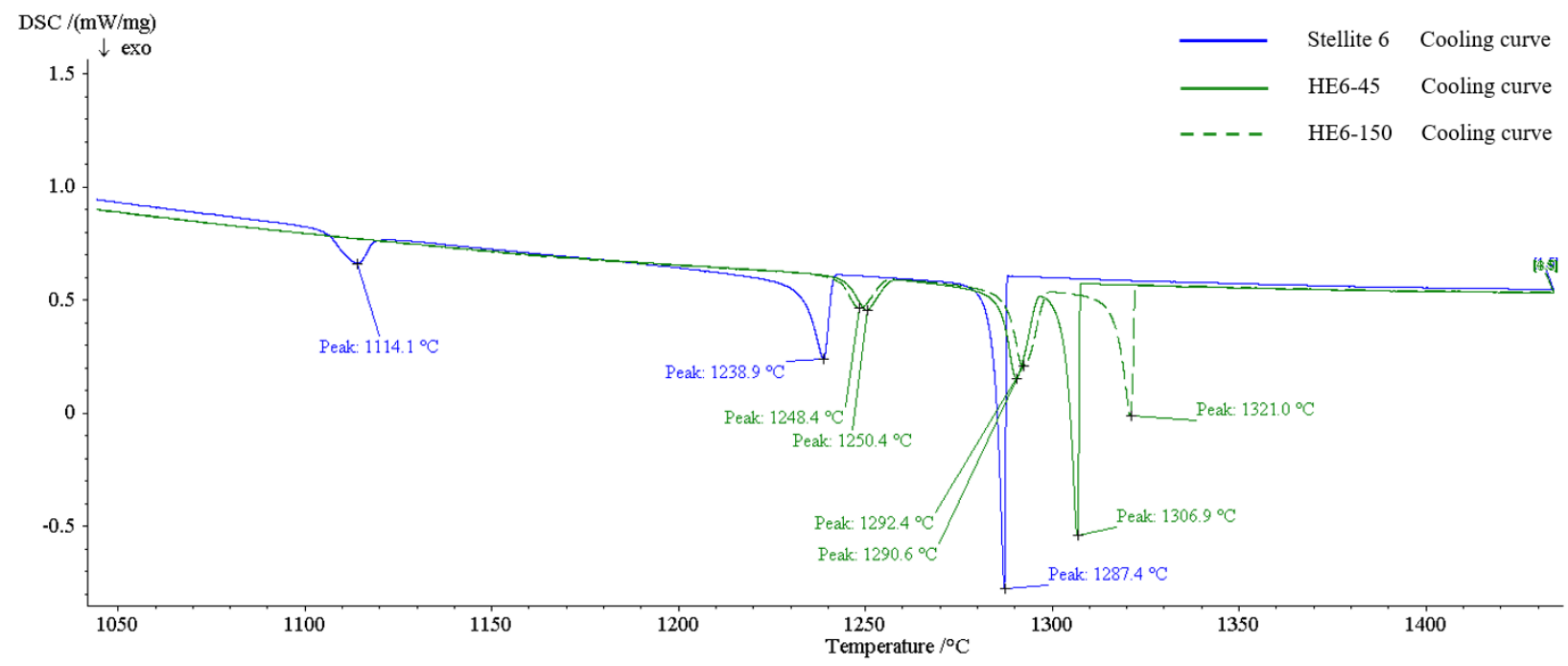

Figure 3-15: DSC cooling curves of tested powders.

\subsection{Bulk Alloy Manufacturing}

Two manufacturing processes were selected to make the bulk alloy specimens of Stellite 6 and HE6 alloy, plasma transferred arc (PTA) welding and spark plasma sintering (SPS). Table 3-5 lists the alloy powders and their manufacturing combinations.

Table 3-5: PTA and SPS specimen completion status.

\begin{tabular}{|c|c|c|c|}
\hline Alloy powder & Size $(\boldsymbol{\mu m})$ & PTA & SPS \\
\hline Stellite 6 & 45 & $\sqrt{ }$ & $\sqrt{ }$ \\
\hline HE6 & 45 & $\sqrt{ }$ & $\sqrt{ }$ \\
\hline HE6 & 150 & N/A & $\sqrt{ }$ \\
\hline
\end{tabular}

\subsubsection{Plasma transferred arc (PTA) welding process}

PTA is a well-established welding process that is used to deposit coating/hardfacing overlays on metal substrates. Kennametal Stellite Inc. produced Stellite 6 and HE6 PTA overlays on 316 stainless steel plates for this research. The substrates were cut off later with wire cutting, and only the overlays were investigated in this study. Table 3-6 gives the manufacturing parameters that were used to prepare the PTA specimens of Stellite 6 and HE6. Wire cutting was 
used to section the PTA specimens into small round or square samples to comply with the requirements of various experiments. Taking one PTA workpiece as an example, Figure 3-16 illustrates the sampling diagram.

Table 3-6: PTA manufacturing parameters.

\begin{tabular}{|c|c|c|}
\hline Process parameter & Stellite 6 & HE6 \\
\hline Overlay thickness $(\mathrm{mm})$ & $4-6$ & $4-6$ \\
\hline Plasma gas flow rate $(1 / \mathrm{min})$ & $2.8-3.0$ & $2.8-3.0$ \\
\hline Powder gas flow rate $(1 / \mathrm{min})$ & $4.5-5.0$ & $4.5-5.0$ \\
\hline Preheat temperature $\left({ }^{\circ} \mathrm{C}\right)$ & 220 & 120 \\
\hline Interpass temperature $\left({ }^{\circ} \mathrm{C}\right)$ & 350 & 250 \\
\hline Post-heat temperature $\left({ }^{\circ} \mathrm{C}\right)$ & 400 & 300 \\
\hline Voltage $(\mathrm{V})$ & $30-35$ & $30-35$ \\
\hline Current $(\mathrm{A})$ & $180-220$ & $180-220$ \\
\hline
\end{tabular}

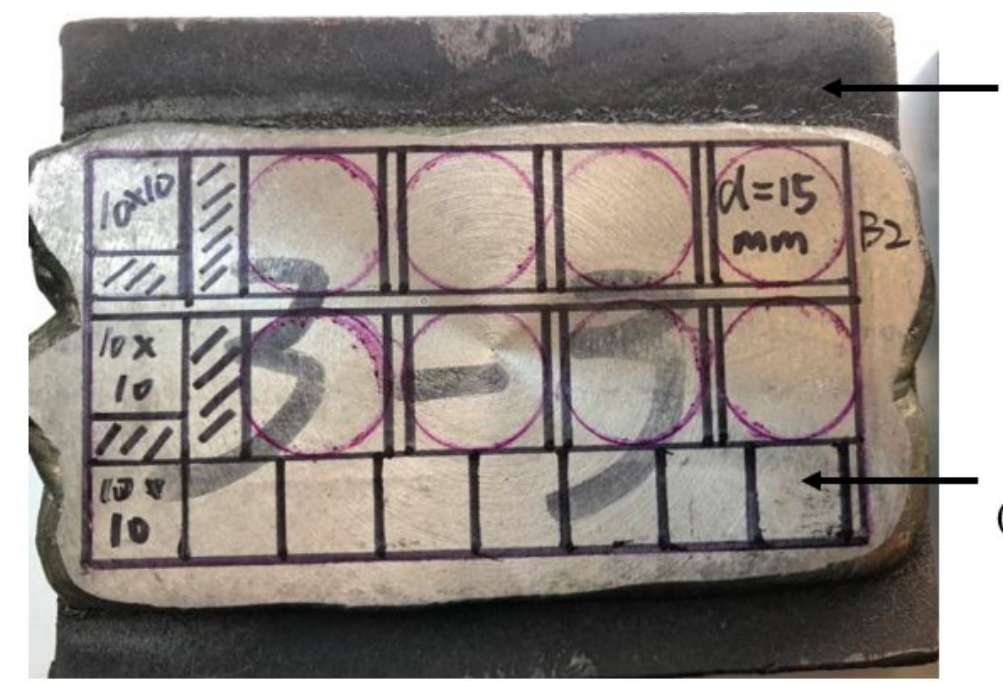

316 stainless steel substrate

PTA overlays (Stellite 6 or HE6)

Figure 3-16: An example of wire cutting on a PTA workpiece.

\subsubsection{Spark plasma sintering (SPS) process}

Meanwhile, SPS has emerged as a promising technique for material sintering process in the recent decade. It has been studied for a wide range of materials, such as ceramic, composites, high-temperature alloys, etc. Compared with other popular sintering techniques, such as cold or hot isostatic pressing pressure (CIP, HIP), SPS provides a much shorter lead time with promising 
product qualities. A Thermal Technology's SPS System Model 10-3 was adopted in this study. A schematic drawing of the SPS process is shown in Figure 3-17 [45]. A weight of 10.56 grams alloy powder was put into round graphite die. The mass was calculated based on the aimed bulk material density and the volume of the die. After the die was placed in the cavity, the chamber was vacuumed to 0.02 torr and then filled with 5.0 grade ultra-high purity argon gas before the start of sintering. A pulsed direct current (DC) power supplied heat to the chamber, while uniaxial pressure was applied and maintained to the die during the whole heating cycle. After an extensive literature review [79]-[83], the manufacturing parameters were chosen for Stellite 6 and HE6, as listed in Table 3-7; and the heating cycle is illustrated in Figure 3-18. Complete information of sample types and sizes are summarized in Table 3-8. In the following analyses and discussion, PTA Stellite $6(45 \mu \mathrm{m})$ or PTA Stellite 6 refers to the bulk specimen from the PTA process using Stellite 6 powder with a particle size of $45 \mu \mathrm{m}$; SPS Stellite $6(45 \mu \mathrm{m})$ or SPS Stellite 6 refers to the bulk specimen from the SPS process using Stellite 6 powder with a particle size of $45 \mu \mathrm{m}$. Similar notion is used for PTA HE6, SPS HE6 $(45 \mu \mathrm{m})$ and SPS HE6 $(150 \mu \mathrm{m})$.

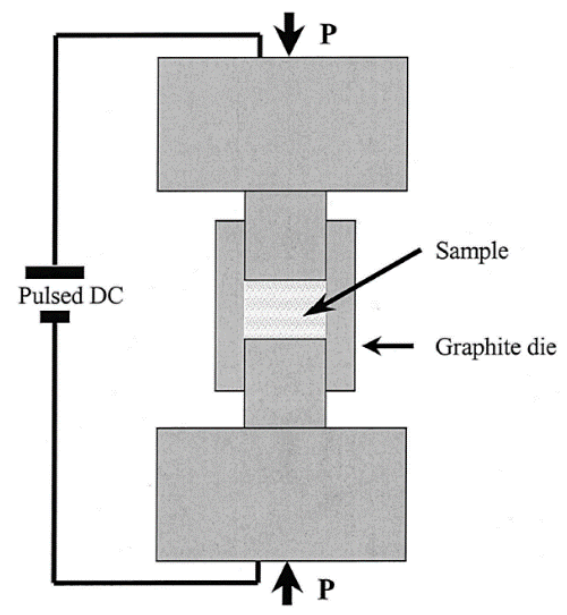

Figure 3-17: Schematic of the working principle of the SPS apparatus [45]. 
Table 3-7: SPS process parameters for alloy specimen manufacturing.

\begin{tabular}{|c|c|}
\hline Process parameter & Stellite 6 \& HE6 \\
\hline Temperature $\left({ }^{\circ} \mathrm{C}\right)$ & 1050 \\
\hline Heating rate $(\mathrm{K} / \mathrm{min})$ & 150 \\
\hline Holding time $(\mathrm{min})$ & 10 \\
\hline Uniaxial pressure $(\mathrm{MPa})$ & 50 \\
\hline Inner diameter of die $(\mathrm{mm})$ & 20 \\
\hline The atmosphere in the chamber & 5.0 grade Ar gas \\
\hline
\end{tabular}

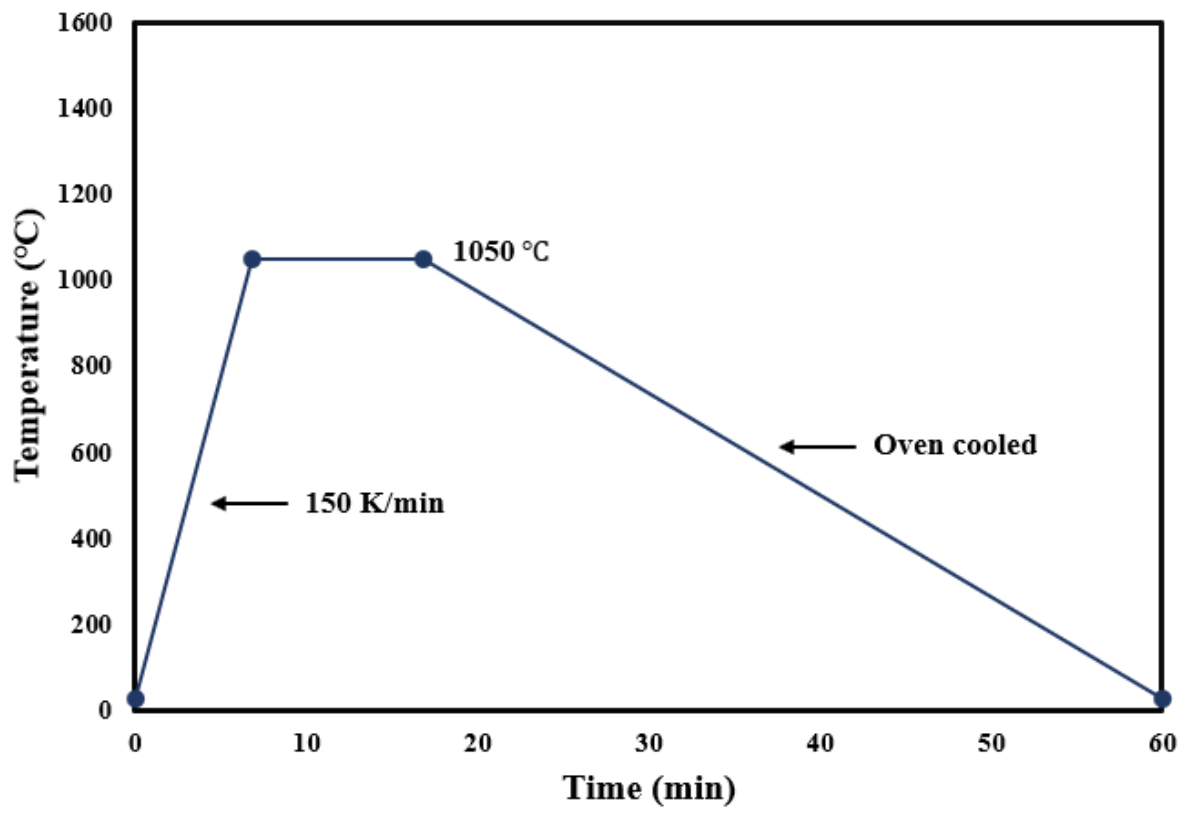

Figure 3-18: SPS heating cycle.

Table 3-8: Bulk specimen manufacturing status and geometry.

\begin{tabular}{|c|c|c|c|c|c|}
\hline \multirow{2}{*}{ Alloy } & \multicolumn{5}{|c|}{ Sample geometry } \\
\hline & $\begin{array}{c}\text { Square } \\
10 \times 10 \mathrm{~mm}\end{array}$ & $\begin{array}{l}\text { Rectangle } \\
20 \times 15 \mathrm{~mm}\end{array}$ & $\begin{array}{l}\text { Round } \\
\emptyset 15 \mathrm{~mm}\end{array}$ & $\begin{array}{l}\text { Round } \\
\varnothing 20 \mathrm{~mm}\end{array}$ & \multirow{6}{*}{$\begin{array}{l}\text { Thickness } \\
\mathrm{t} \approx 5 \mathrm{~mm}\end{array}$} \\
\hline PTA Stellite 6 & $\sqrt{ }$ & $\sqrt{ }$ & $\sqrt{ }$ & \multirow{2}{*}{$\mathrm{N} / \mathrm{A}$} & \\
\hline PTA HE6 $(45 \mu \mathrm{m})$ & $\sqrt{ }$ & $\sqrt{ }$ & $\sqrt{ }$ & & \\
\hline SPS Stellite 6 & \multirow{3}{*}{\multicolumn{3}{|c|}{ N/A }} & $\sqrt{1}$ & \\
\hline SPS HE6 $(45 \mu \mathrm{m})$ & & & & $\sqrt{ }$ & \\
\hline SPS HE6 $(150 \mu \mathrm{m})$ & & & & $\sqrt{ }$ & \\
\hline
\end{tabular}




\subsubsection{Density measurement of bulk alloy specimens}

The densities of bulk alloy specimens were calculated by dividing the mass by the volume. Three specimens of each alloy were measured for average mass and volume. Moreover, the porosity of each specimen was calculated via the image processing method. The obtained density results are summarized in Table 3-9. Higher density reflects better internal consolidation in the manufacturing process, which contributes to the mechanical properties of the material and the integrity of powders. The density of fully dense Stellite 6 is $8.34 \mathrm{~g} / \mathrm{cm}^{3}$ which is the actual data of cast Stellite 6. Compared with the official data of cast Stellite 6, both the PTA and SPS Stellite 6 specimens are close to the fully dense state, with the former having a relative density of $99.0 \%$ and the latter $97.6 \%$. It can be inferred that Stellite 6 specimens have been successfully manufactured with PTA and SPS processes. PTA HE6 $(45 \mu \mathrm{m})$ has a higher density of $8.915 \mathrm{~g} / \mathrm{cm}^{3}$ which is attributed to its higher content of W. Since HE6 is newly designed, there is no available density data. But based on that the PTA specimen is close to the casting state, its density is considered as a fully dense value. The SPS HE6 specimens have close density values to the PTA ones. The 96.7\% and $98.5 \%$ relative density of SPS HE6 $(45 \mu \mathrm{m})$ and SPS HE6 $(150 \mu \mathrm{m})$ indicate that the bulk specimens have been fully compacted via the SPS process.

Table 3-9: Density and porosity measurements of alloy specimens.

\begin{tabular}{|c|c|c|c|c|}
\hline \multirow{2}{*}{ Alloy } & \multicolumn{2}{|c|}{ PTA } & \multicolumn{2}{c|}{ SPS } \\
\cline { 2 - 5 } & Density $\left(\mathbf{g} / \mathbf{c m}^{\mathbf{3}}\right)$ & Relative density & Density $\mathbf{( g / \mathbf { c m } ^ { 3 } )}$ & Relative density \\
\hline Stellite 6 & $8.258 \pm 0.011$ & $99.0 \%$ & $8.141 \pm 0.014$ & $97.6 \%$ \\
\hline HE6 (45 $\boldsymbol{\mu m} \mathbf{m})$ & $8.915 \pm 0.032$ & 1 & $8.621 \pm 0.063$ & $96.7 \%$ \\
\hline HE6 (150 $\boldsymbol{\mu m})$ & \multicolumn{2}{|c|}{ N/A } & $8.757 \pm 0.130$ & $98.5 \%$ \\
\hline
\end{tabular}




\subsection{Sample Surface Preparation}

For the following microstructural analyses and performance evaluations, the surfaces of alloy samples were prepared with the procedures as follows:

1) The alloy samples were hot mounted before polishing with the Simplimet II Mounting Machine (Figure 3-19) using phenolic resin.

2) The sample was ground and polished using a Buehler MetaServ ${ }^{\mathrm{TM}} 250$ Grinder-Polishing Machine (Figure 3-20). The polishing took steps with a series grit of sandpapers, 180, 240, $320,400,600,800$ and 1200 grit, from coarse to fine; followed by a step with $3 \mu \mathrm{m}$ diamond suspension polish; then a final step was taken to achieve a mirror-like surface using $0.06 \mu \mathrm{m}$ colloidal silica suspension. The surface finish requirements vary for different tests.

3) Table 3-10 summarizes the requirements used for all the tests in this study.

Table 3-10: A summary of the surface requirements for various experiments.

\begin{tabular}{|c|c|}
\hline Test & Surface finish requirement \\
\hline SEM/EDX & $0.06 \mu \mathrm{m}$ final polishing \\
\hline Polarization & $0.06 \mu \mathrm{m}$ final polishing \\
\hline Microhardness & $0.06 \mu \mathrm{m}$ final polishing \\
\hline Immersion & Up to 1200 grit \\
\hline Pin-on-disc wear & Up to 400 grit \\
\hline X-ray diffraction (XRD) & Up to 400 grit \\
\hline
\end{tabular}




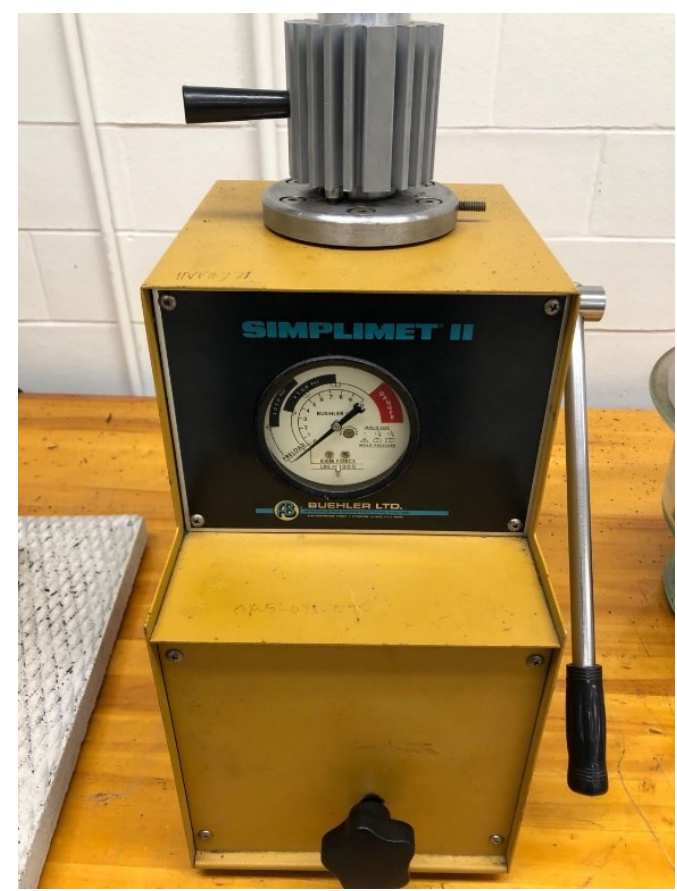

Figure 3-19: Buehler Simplimet II Mounting Press.

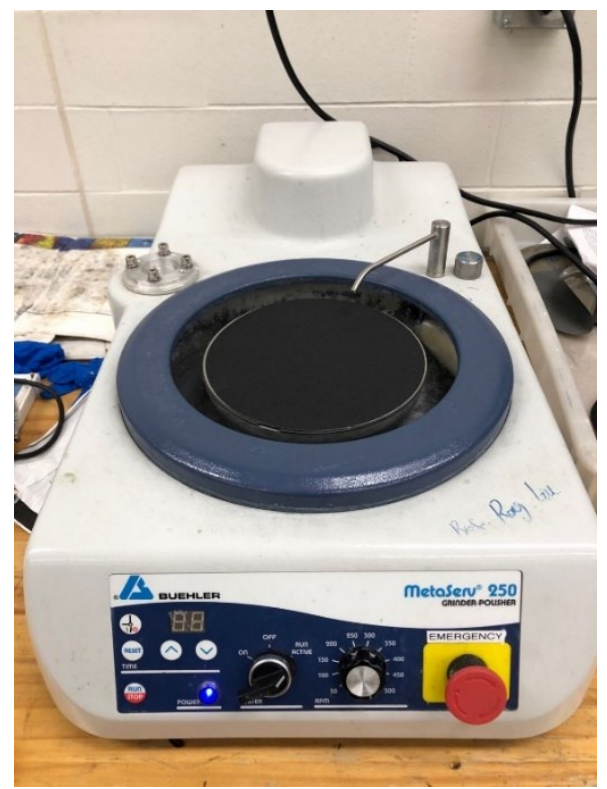

Figure 3-20: Buehler MetaServ ${ }^{\mathrm{TM}} 250$ Grinder-Polisher. 
4) The sample was cleaned up with an ultrasonic cleaner for 15 minutes (Figure 3-21) after polishing. It was also used to clean up the sample surface after wear and corrosion tests.

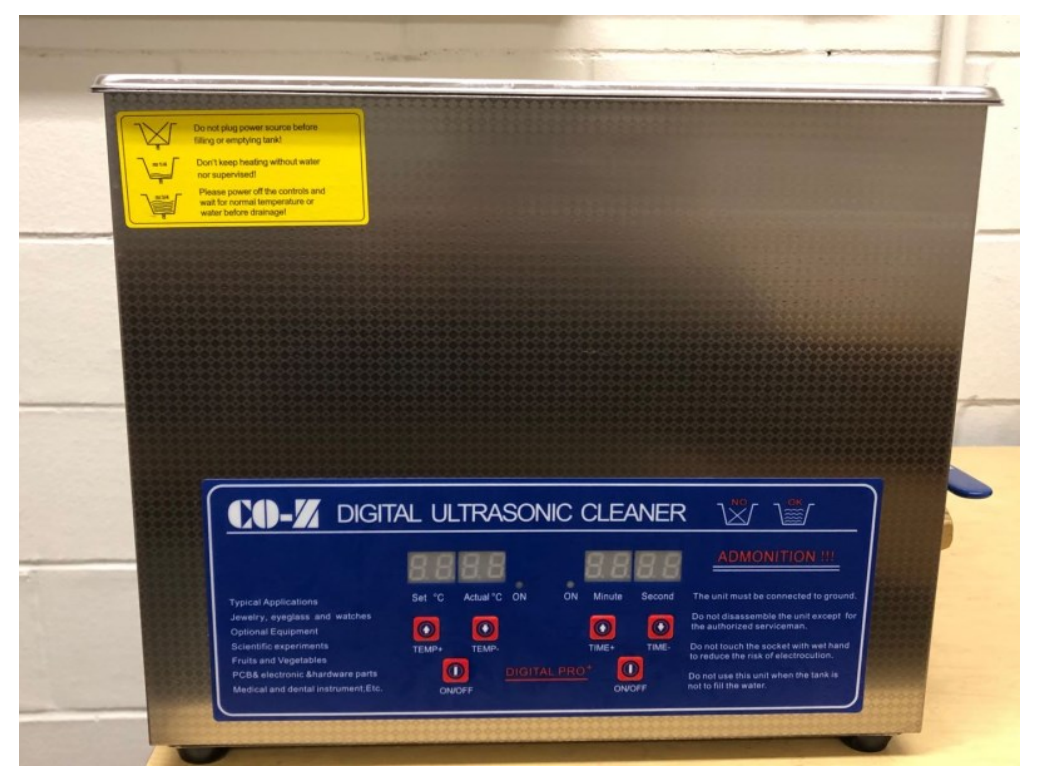

Figure 3-21: CO-Z 6L Professional Ultrasonic Cleaner. 


\subsection{Microstructural Analysis of Bulk Alloys}

The microstructures of bulk HE6 and Stellite 6 alloy specimens from PTA and SPS processes were analyzed using a scanning electron microscope (SEM), equipped with an energy dispersive X-ray spectroscopy (EDX) unit, with the assistance of X-ray diffraction (XRD). The combination of these techniques is able to analyze the microstructure morphology, identify phases present and quantify main constituents in the microstructure.

\subsubsection{XRD analysis}

XRD was utilized to generate a scanning pattern for both the bulk specimens and powder samples of HE6 and Stellite 6 alloy, in order to investigate the phase transformation that had occurred in HE6 and Stellite 6 alloy during the PTA and SPS processes, converting the alloy powders to bulk specimens.

The Rigaku Ultima IV Diffractometer (X-ray Core Facility, University of Ottawa) was employed to obtain the diffraction patterns in the present study. The theta-theta system with copper radiation $(\mathrm{Cu} \mathrm{K} \alpha)$ and one diffracted beam monochromator was applied to scan the sample surface from $30^{\circ}$ to $100^{\circ}$. Then the XRD patterns were interpreted with the analytical software, JADE 9 Plus (version 9.8.0), equipped with Powder Diffraction File (PDF) ICDD database 2020. Each phase in a microstructure has its identical peaks in the database so that the obtained peak patterns from the scan on the sample were carefully compared with the standards of phase peaks to identify each phase in the examined microstructure. 


\subsubsection{XRD patterns of Stellite 6}

The combined XRD patterns of Stellite 6 alloy in various forms are presented in Figure 3-22, demonstrating similarities and differences in phases of Stellite 6 under various manufacturing conditions.

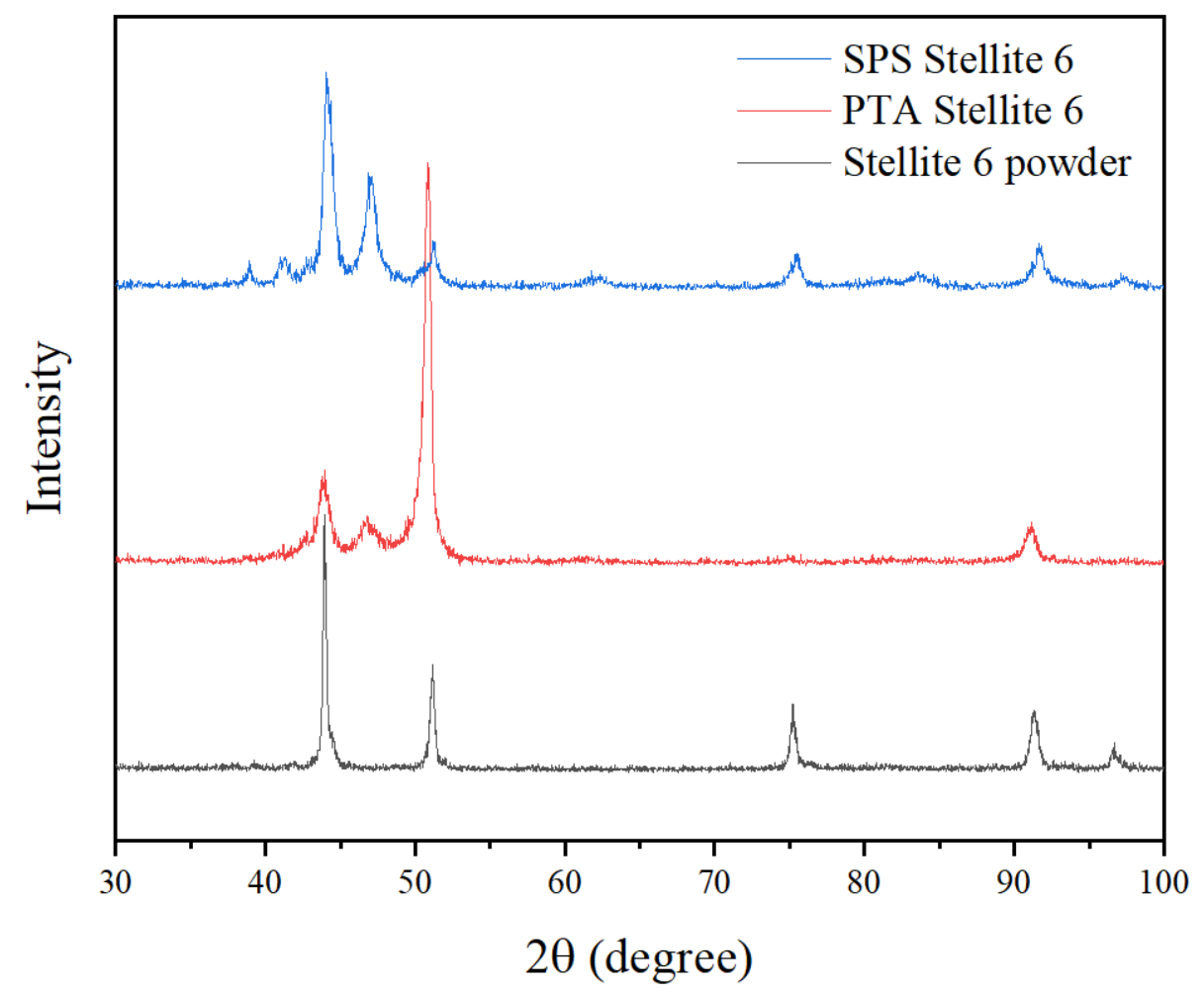

Figure 3-22: Combined XRD patterns of Stellite 6 alloy in powder and bulk form.

The XRD pattern of Stellite 6 powder with the identified phases is illustrated in Figure 3-23. In general, it well agrees with literature findings [79]. The solid solution is Co phase in FCC structure. The major precipitates are confirmed to be complex $\mathrm{M}_{23} \mathrm{C}_{6}$ carbides, where $\mathrm{M}$ represents the mixing of $\mathrm{Cr}$ and $\mathrm{Fe}$. The XRD results also find some intermetallics existing at the major peaks such as $\mathrm{FeNi}_{3}, \mathrm{Cr}_{0.095} \mathrm{Fe}_{0.38} \mathrm{Co}_{0.528}, \mathrm{Cr}_{4} \mathrm{Ni}_{15} \mathrm{~W}$ and $\mathrm{Co}_{3} \mathrm{Mo}$. 


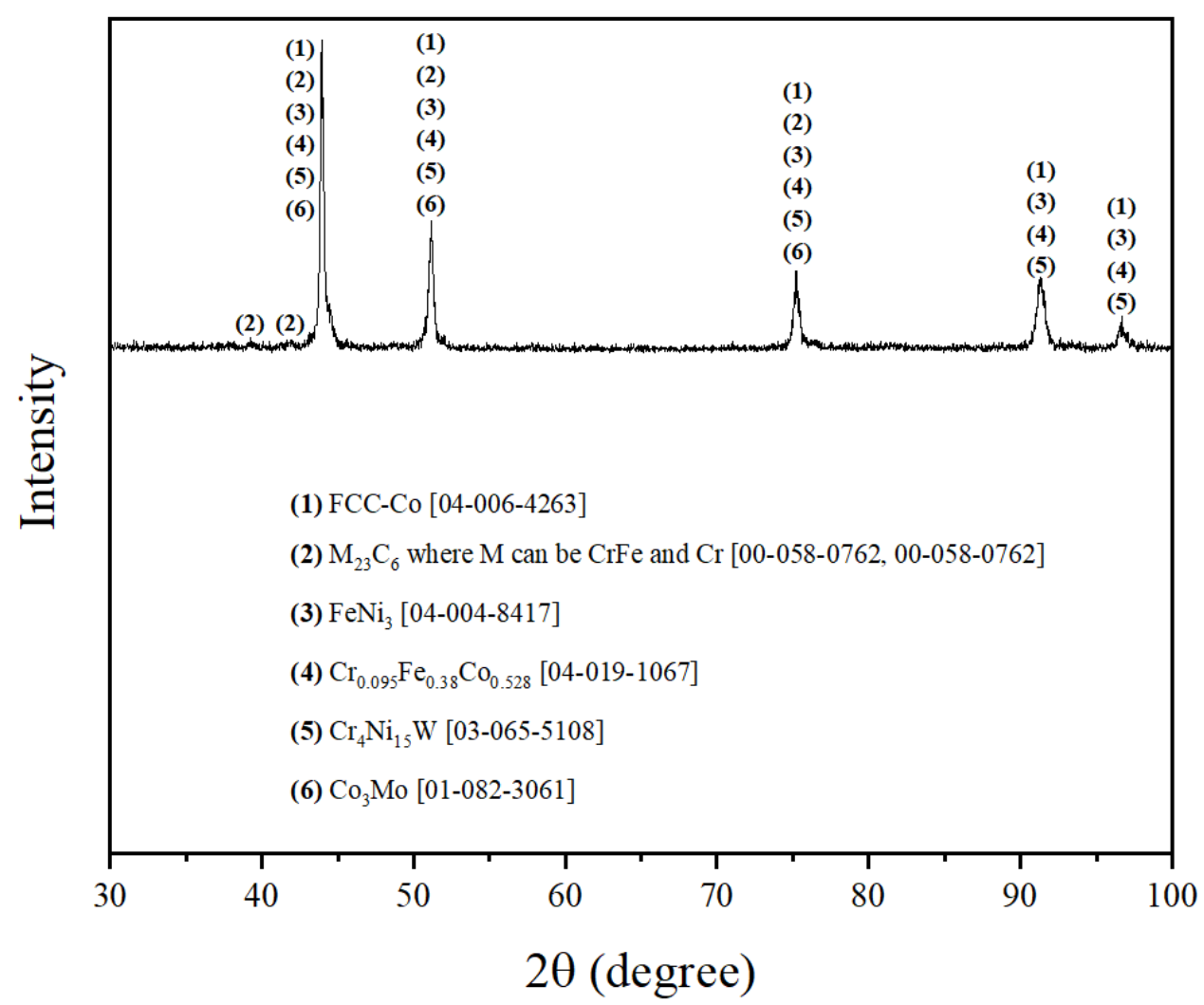

Figure 3-23: XRD pattern and phase identification of Stellite 6 powder.

Figure 3-24 illustrates the XRD results of PTA Stellite 6. Compared to the powder form, the peaks of HCP (hexagonal close-packed) Co phase are detected in PTA Stellite 6, along with some new intermetallics formed after welding, as indicated in the XRD pattern. Cobalt has a stable HCP structure at low temperature, but when the temperature rises, HCP to FCC transition occurs around $700 \mathrm{~K}$. This type of phase transition is generally sluggish [84]. Stellite alloys usually contain different proportions of $\mathrm{HCP} \mathrm{Co}$, especially after hot working and slow cooling processes. Regarding carbides, the PTA process did not change them very much; there are still $\mathrm{M}_{23} \mathrm{C}_{6}$ dominating in the PTA specimen. For intermetallics, more complex combinations have emerged in the PTA form of Stellite 6. 


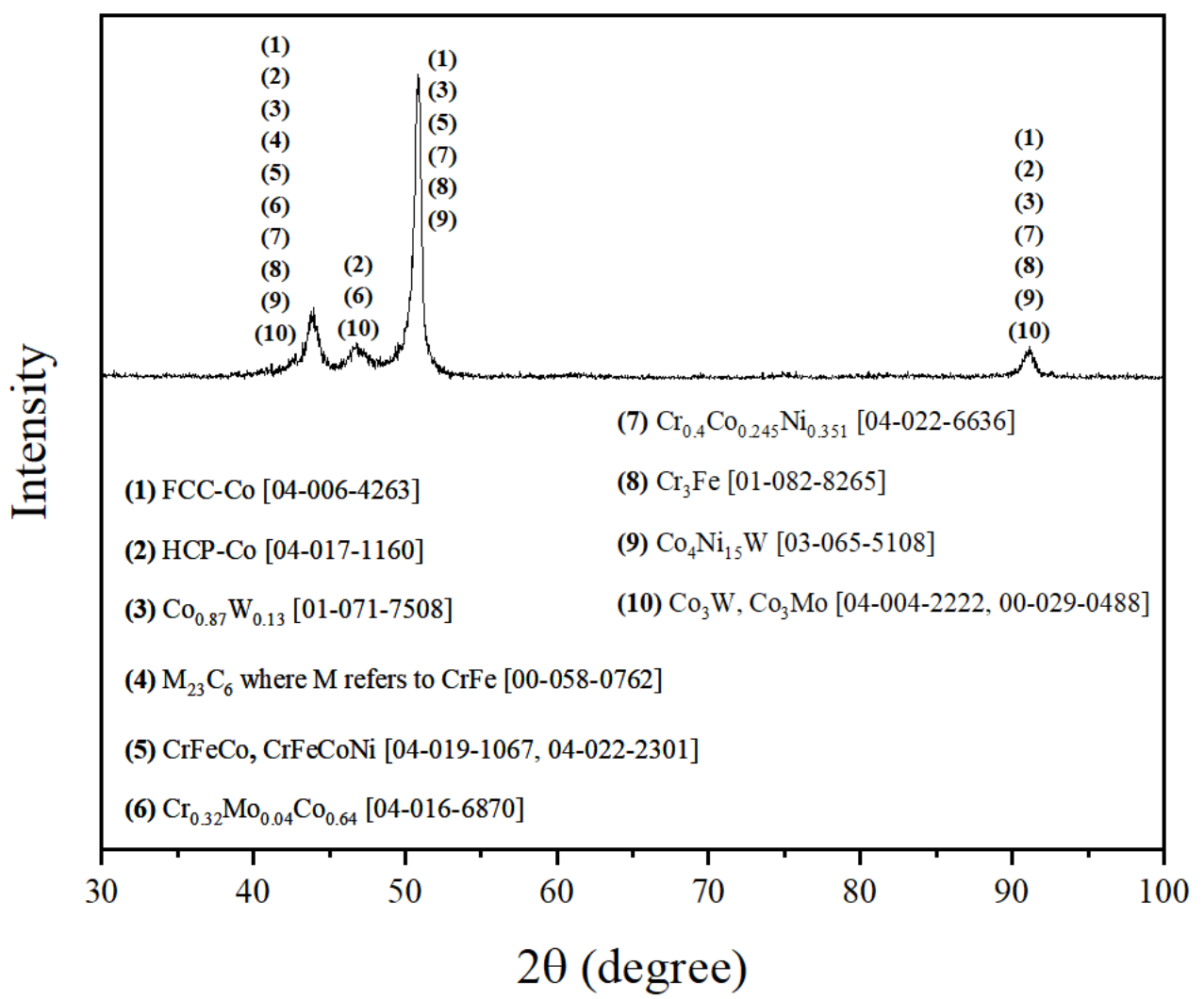

Figure 3-24: XRD pattern and phase identification of PTA Stellite 6.

As for SPS Stellite 6, the profile of the peaks is shown in Figure 3-25. Similar to the PTA specimen, $\mathrm{HCP}$ Co is present in the bulk specimen from SPS process. In addition to $\mathrm{M}_{23} \mathrm{C}_{6}$ carbides, Fe or $\mathrm{W}$ containing $\mathrm{M}_{2} \mathrm{C}$ carbides are also detected. However, it is interesting to note that no intermetallics are found in this specimen according to the XRD pattern. 


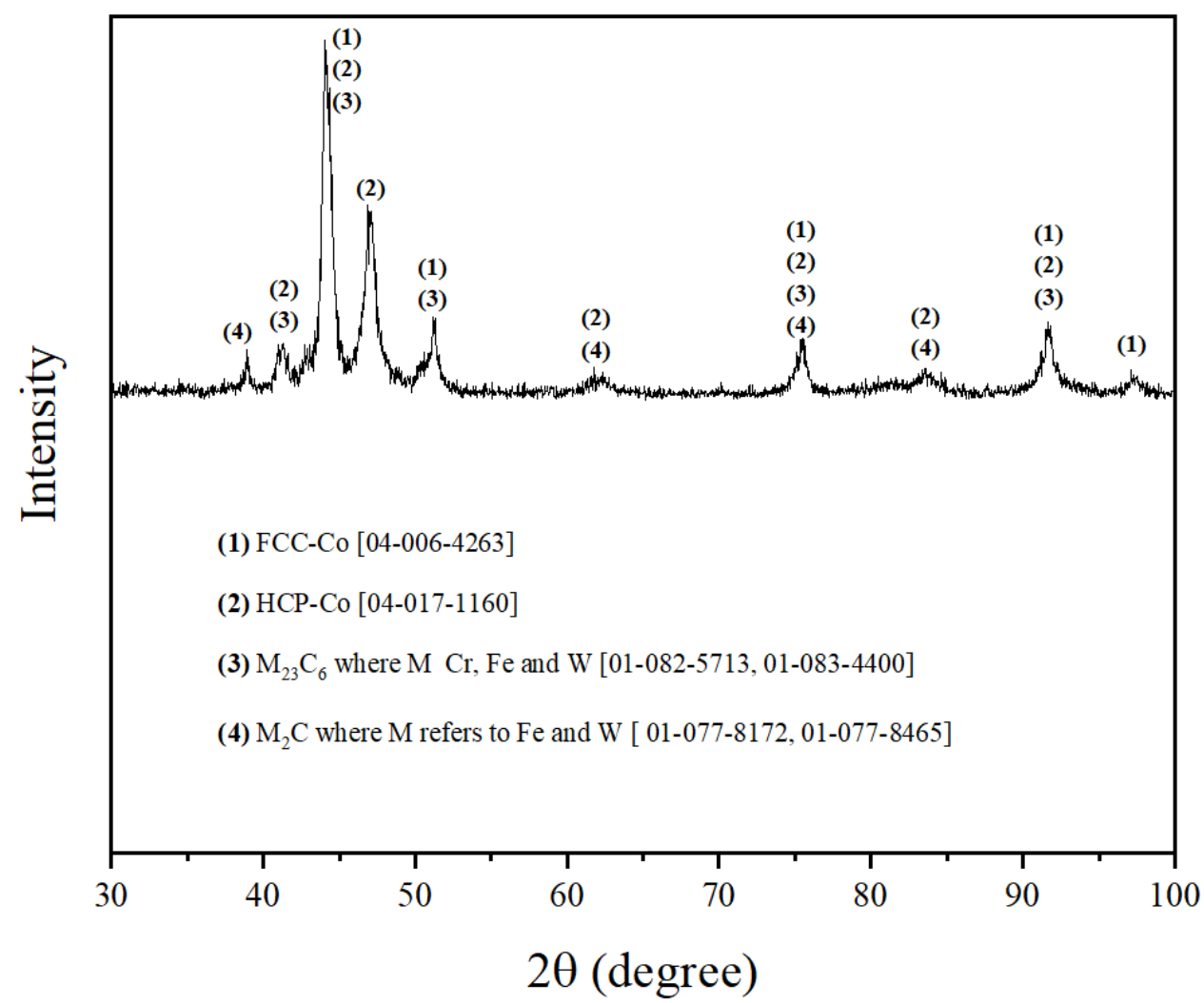

Figure 3-25: XRD pattern and phase identification of SPS Stellite 6.

\subsubsection{XRD patterns of HE6}

Figure 3-26 presents the XRD patterns of HE6 powders with different particle sizes, and Figure 3-27 shows the XRD patterns of HE6 bulk specimens from PTA and SPS processes. It is observed that the HE6 powders with large and small particle sizes have identical phases. For the bulk specimens, in general, the XRD patterns are similar. Thus, one XRD pattern from the powder group and one from the bulk specimen group were taken for detailed phase analysis. 


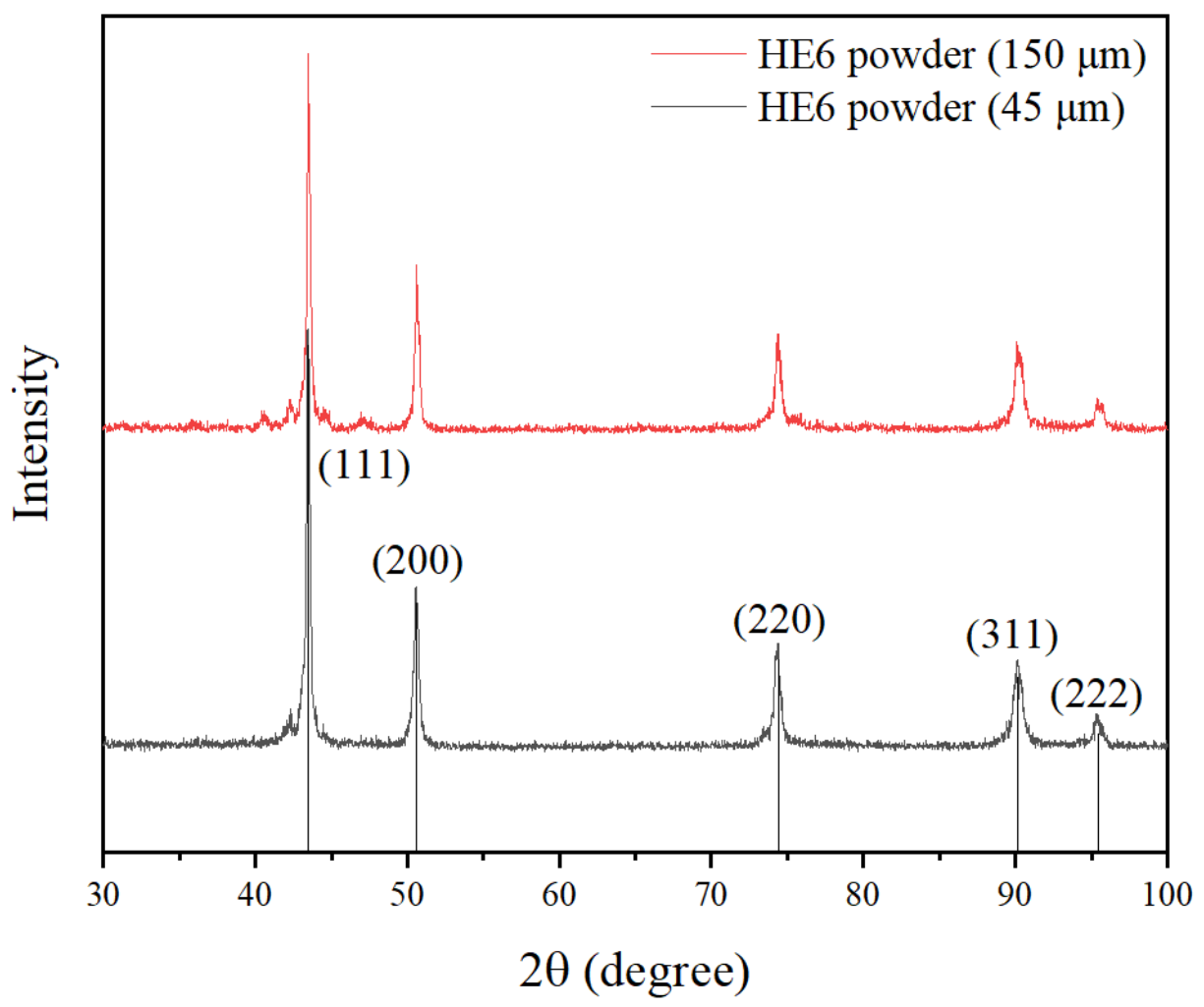

Figure 3-26: Combined XRD patterns of HE6 powders with different particle sizes.

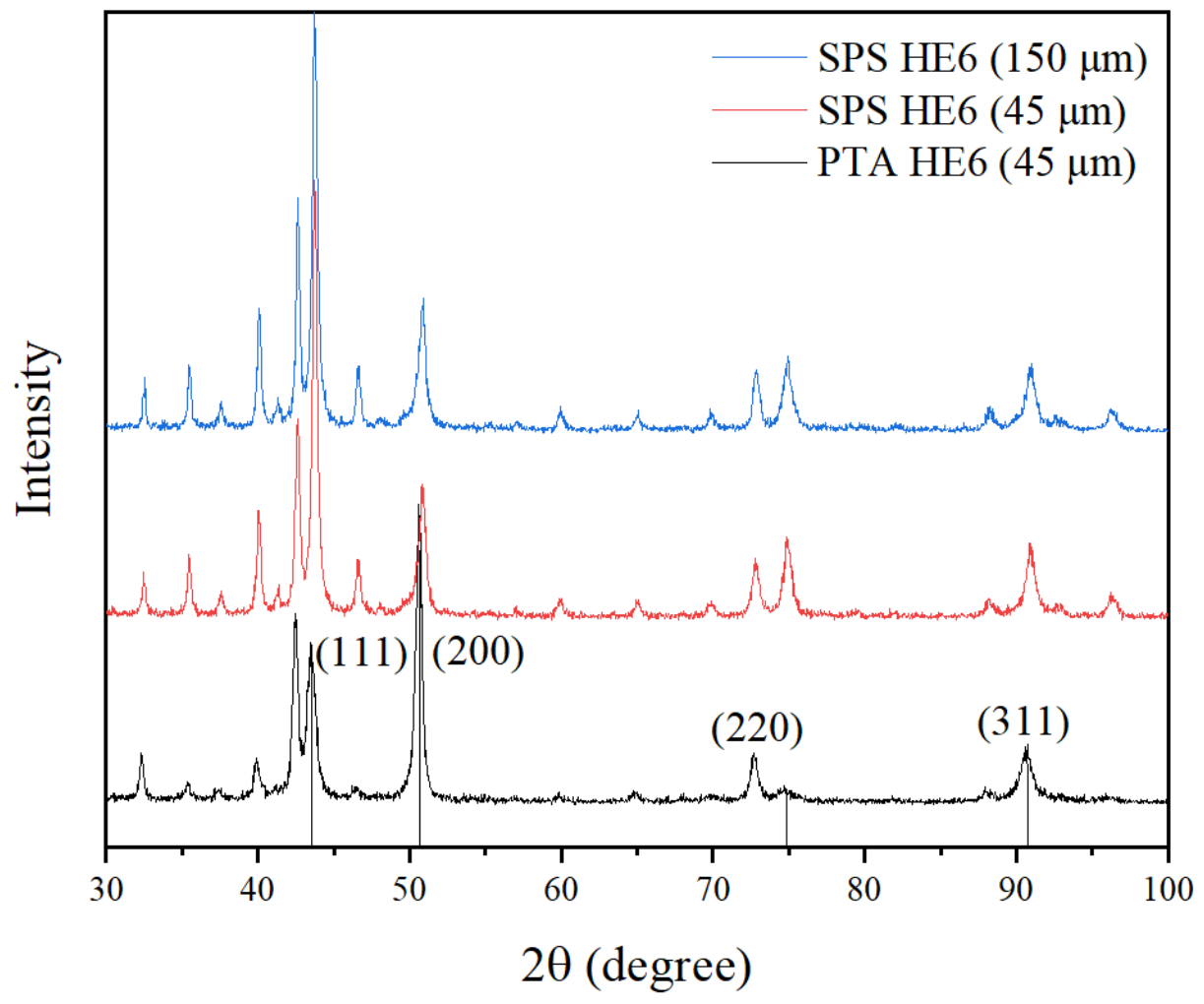

Figure 3-27: Combined XRD patterns of HE6 bulk specimens from SPS and PTA processes. 
The XRD results of HE6 powder $(150 \mu \mathrm{m})$ and SPS HE6 $(150 \mu \mathrm{m})$ bulk specimens are presented in Figure 3-28 and Figure 3-29, respectively. Using the phase analysis software, FCC phase is confirmed to be the solid solution of HE6 in both powder and bulk form. The phases in this alloy are identified to be much more complex, compared to that of Stellite 6 . This should be attributed to the multi-element system (Co-Cr-Fe-Ni) of HE6, rather than a single major element Co of Stellite 6. The FCC solid solution of HE6 consists of $\mathrm{Co}, \mathrm{Cr}, \mathrm{Fe}$ and Ni. Three types of carbides with different contents are detected in HE6 powder and bulk forms: $\mathrm{MC}, \mathrm{M}_{16} \mathrm{C}$ and $\mathrm{M}_{23} \mathrm{C}$ for HE6 powders; $\mathrm{M}_{6} \mathrm{C}, \mathrm{M}_{16} \mathrm{C}$ and $\mathrm{M}_{23} \mathrm{C}$ for $\mathrm{HE} 6$ bulk specimens. Several complex intermetallics are also found in both powder and bulk forms. Further analyses in the microstructures of bulk Stellite 6 and HE6 specimens were performed with SEM and EDX.

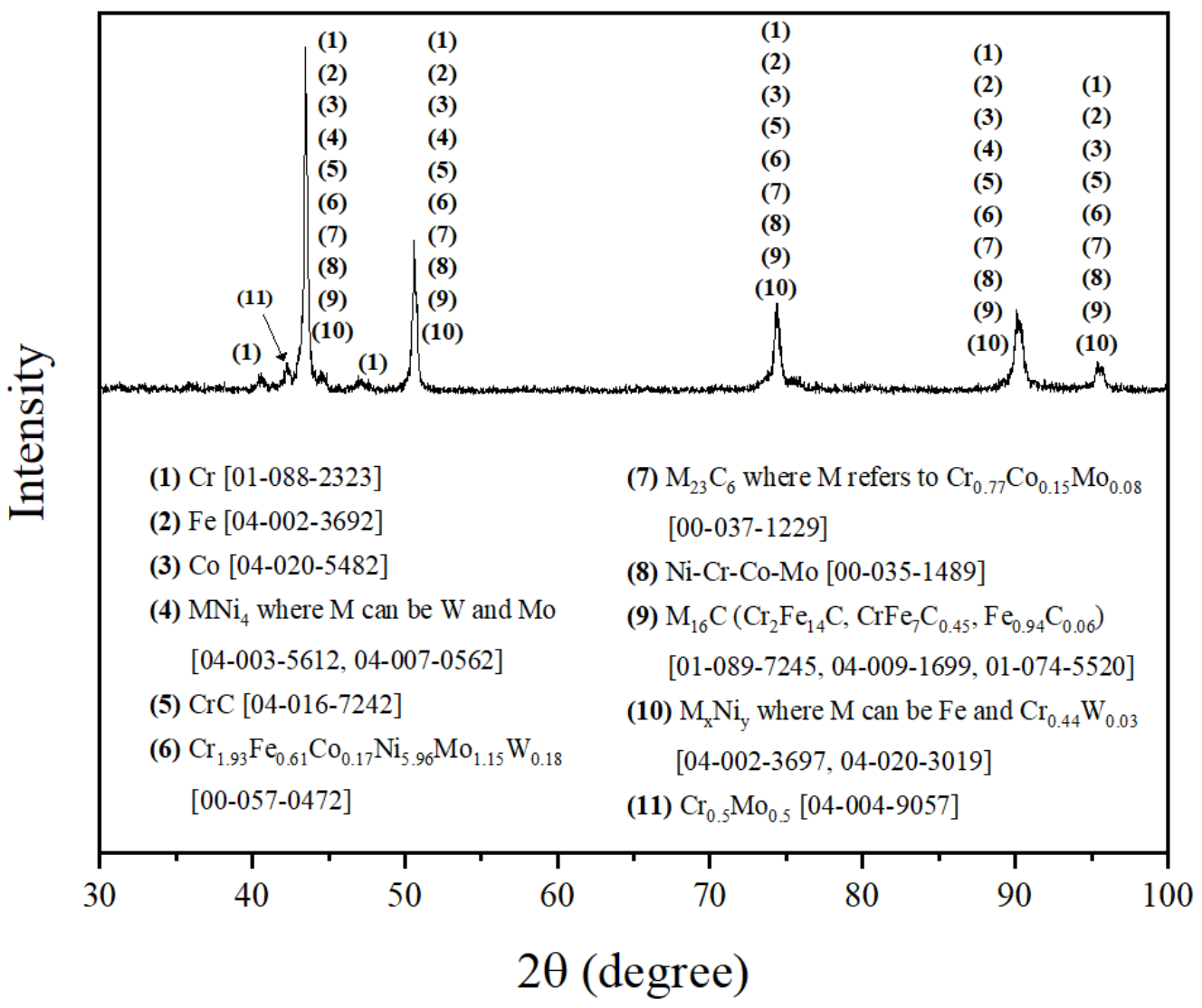

Figure 3-28: XRD pattern and phase identification of HE6 powder $(150 \mu \mathrm{m})$. 


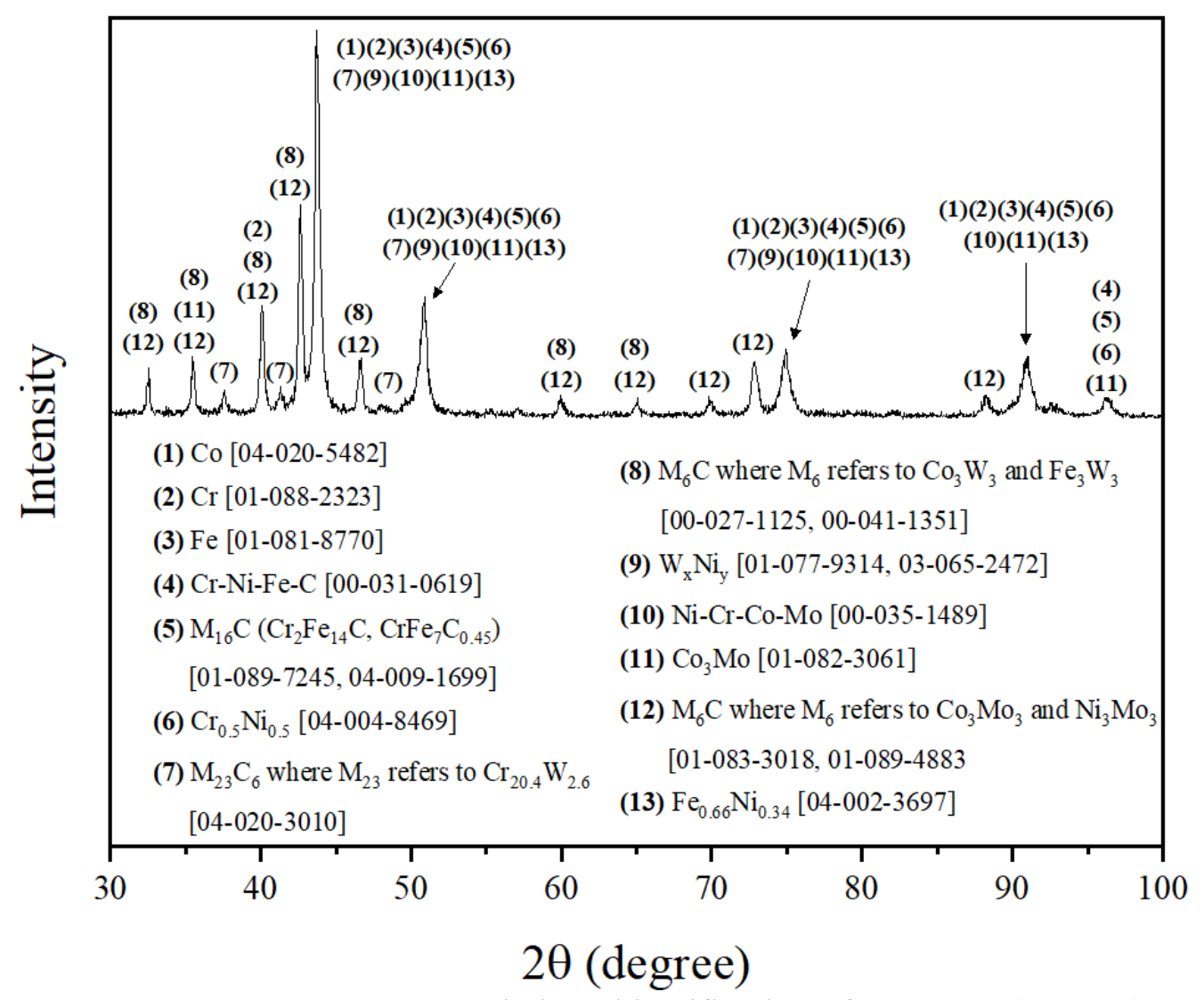

Figure 3-29: XRD pattern and phase identification of SPS HE6 $(150 \mu \mathrm{m})$.

\subsubsection{SEM and EDX analyses}

SEM/EDX techniques enable analyzing the morphology and compositions of the material surface at the same time. EDX provides an accurate quantitative analysis of the chemical composition in a target area. However, light elements such as carbon and oxygen are rather qualitative due to their low energy level. Thus, they are difficult to be quantified by EDX. The elements after oxygen in the periodic table are considered to be reliable in EDX quantification.

\subsubsection{Microstructure of PTA Stellite 6}

The PTA Stellite 6 specimen has the typical hypoeutectic microstructure that consists of various carbides dispersed in a Co-based solid solution matrix, as shown in Figure 3-30. A higher magnification image of the specimen surface in Figure 3-31 details the morphology of carbides 
and solid solution in the alloy microstructure, which also marks the areas for EDX analysis. The EDX results show quantitative differences in chemical composition between the examined areas, as reported in Table 3-11. According to the elemental concentrations, together with the XRD results, the grey area, which is the main constituent of the microstructure, should be Co-based solid solution because of very high $\mathrm{Co}$ content. The dark area is $\mathrm{Cr}$-rich carbides due to high $\mathrm{Cr}$ and $\mathrm{C}$ contents. The bright area is W/Mo-rich intermetallics since it contains high $\mathrm{W}$ content and relatively high Mo content. These results agree with the findings of previous research reported in the literature [85].

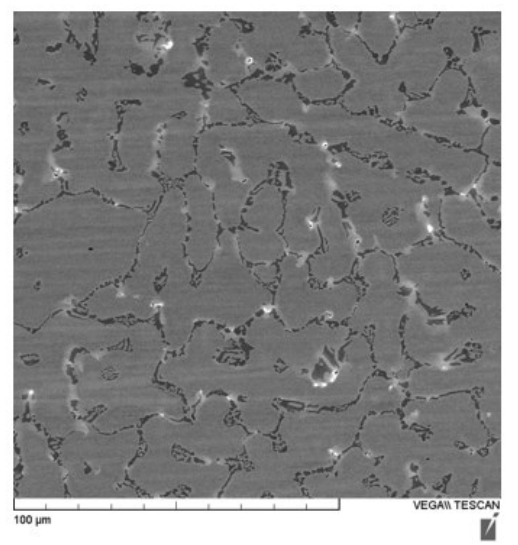

(a)

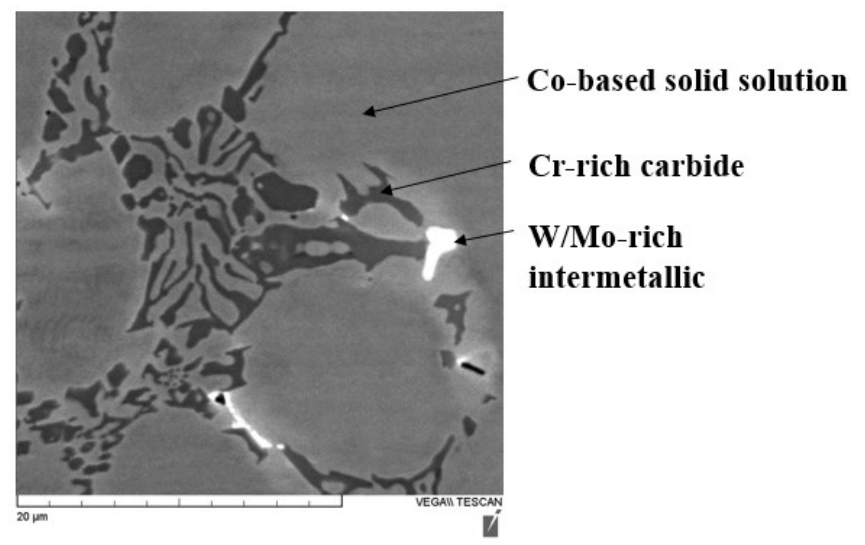

(b)

Figure 3-30: PTA Stellite 6 microstructure (a) at low magnification, (b) at high magnification. 


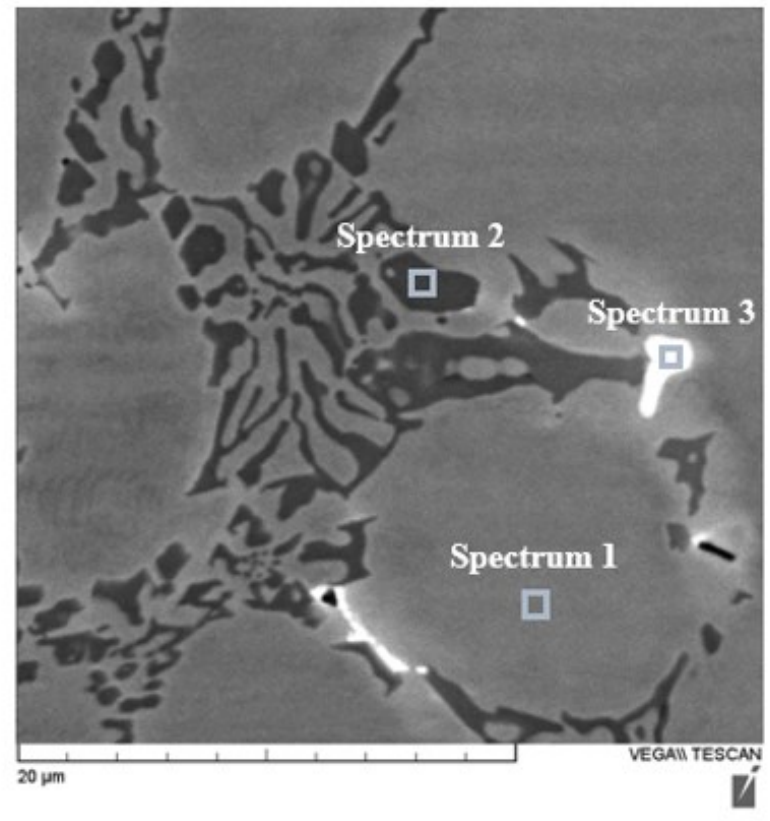

(a)
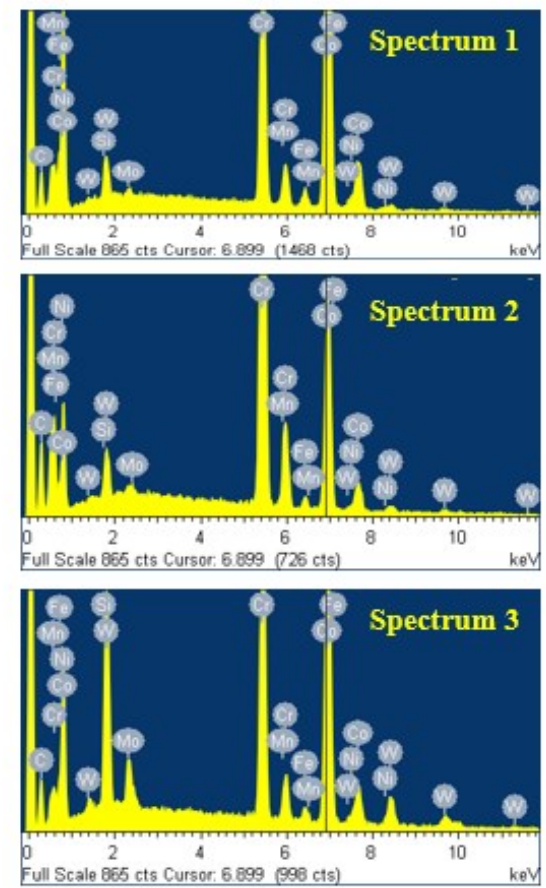

(b)

Figure 3-31: EDX spectra of PTA Stellite 6 microstructure: (a) locations of spectrum, (b) spectrum 1 of grey area (Co-based solid solution), spectrum 2 of dark area (Cr-rich carbides), spectrum 3 of bright area (W/Mo-rich intermetallics).

Table 3-11: EDX elemental table of PTA Stellite 6.

\begin{tabular}{|c|c|c|c|c|c|c|c|c|}
\hline \multicolumn{3}{|c|}{ Grey (Co-based solid solution) } & \multicolumn{3}{|c|}{ Dark (Cr-rich carbide) } & \multicolumn{3}{|c|}{ Bright (W/Mo-rich intermetallic) } \\
\hline Element & wt.\% & at. $\%$ & Element & wt.\% & at.\% & Element & wt.\% & at.\% \\
\hline $\mathrm{C}$ & 3.50 & 14.86 & $\mathrm{C}$ & 16.71 & 48.55 & $\mathrm{C}$ & 13.00 & 45.25 \\
\hline $\mathbf{S i}$ & 0.91 & 1.64 & Si & 0.19 & 0.24 & $\mathbf{S i}$ & 0.78 & 1.15 \\
\hline $\mathrm{Cr}$ & 30.09 & 29.50 & $\mathrm{Cr}$ & 48.51 & 32.55 & $\mathrm{Cr}$ & 23.75 & 19.11 \\
\hline $\mathbf{F e}$ & 2.03 & 1.85 & Mn & 0.03 & 0.02 & Mn & 0.07 & 0.06 \\
\hline Co & 57.19 & 49.46 & $\mathbf{F e}$ & 1.12 & 0.70 & $\mathbf{F e}$ & 1.25 & 0.94 \\
\hline $\mathbf{N i}$ & 1.35 & 1.17 & Co & 27.68 & 16.39 & Co & 37.78 & 26.81 \\
\hline Mo & 0.62 & 0.33 & $\mathbf{N i}$ & 0.81 & 0.48 & $\mathbf{N i}$ & 1.03 & 0.74 \\
\hline $\mathbf{W}$ & 4.33 & 1.20 & Mo & 0.75 & 0.27 & Mo & 4.17 & 1.82 \\
\hline & & & $\mathbf{W}$ & 4.20 & 0.80 & $\mathbf{W}$ & 18.17 & 4.13 \\
\hline
\end{tabular}




\subsubsection{Microstructure of SPS Stellite 6}

Different from PTA Stellite 6 specimen which has the casting microstructure, the SPS Stellite 6 specimen has a powder metallurgy (PM) microstructure in which fine precipitates (dark region) are distributed uniformly over the solid solution (grey region), rather than forming dendritic structures, as shown in Figure 3-32. Moreover, the W/Mo-rich bright phase (intermetallics) in PTA Stellite 6 is not found in this SPS version. Instead, these intermetallics are replaced by other carbides according to the XRD results. The EDX results, reported in Figure 3-33 and Table 3-12, reveal that the dark precipitates are Cr-rich carbides because of high $\mathrm{Cr}$ and $\mathrm{C}$ contents, while the gray area is Co-based solid solution owing to high Co content. Additionally, it is noteworthy that a type of fine black precipitates is found in the microstructure of SPS Stellite 6 which refers to $\mathrm{M}_{2} \mathrm{C}$, where $\mathrm{M}$ represents $\mathrm{W}$ or $\mathrm{Fe}$, according to the XRD results. The presence of $\mathrm{M}_{2} \mathrm{C}$ (mainly $\mathrm{W}_{2} \mathrm{C}$ ) implies that the hardness of this alloy may rise significantly after the SPS process.

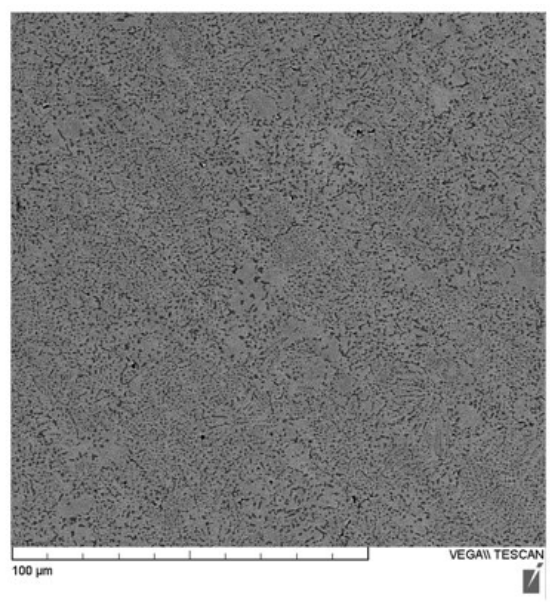

(a)

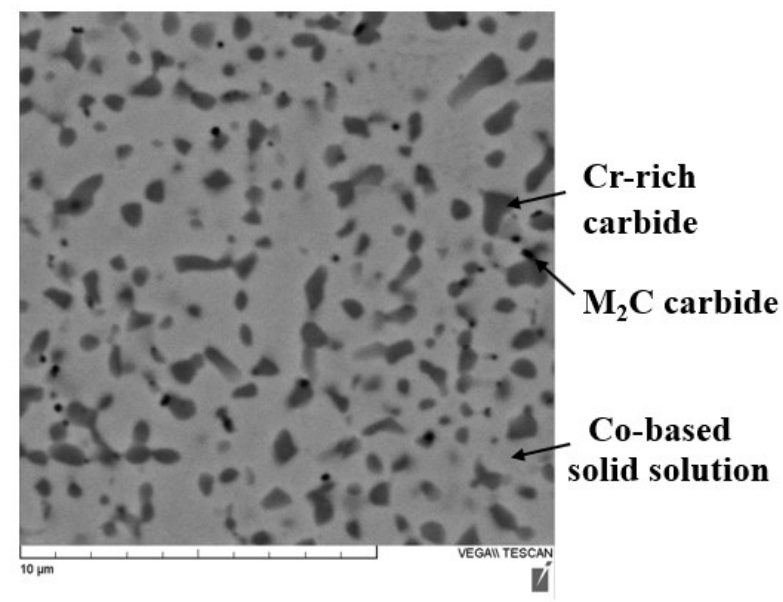

(b)

Figure 3-32: SPS Stellite 6 microstructure (a) at low magnification, (b) at high magnification. 


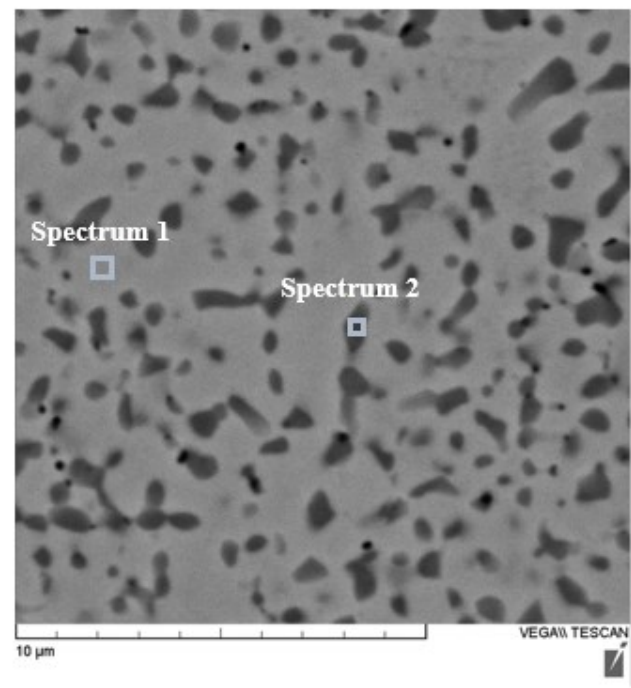

(a)
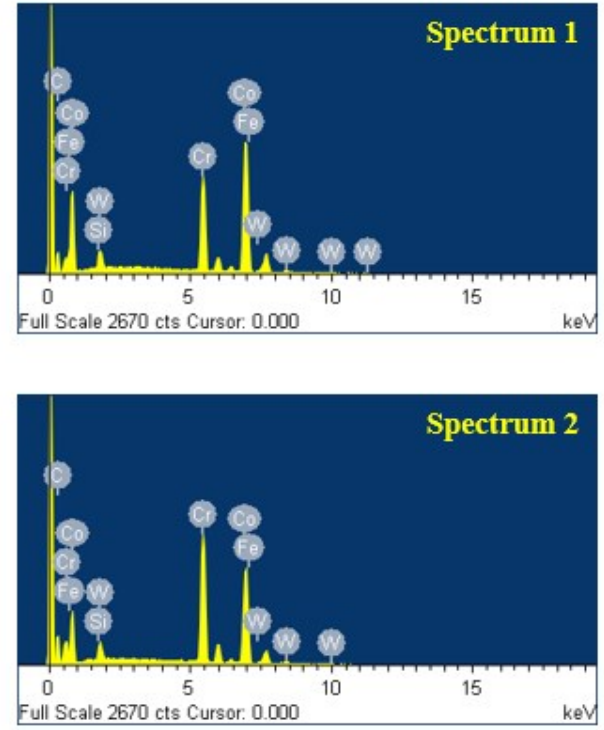

(b)

Figure 3-33: EDX spectra of SPS Stellite 6 microstructure: (a) locations of spectrum, (b) spectrum 1 of grey area (Co-based solid solution), spectrum 2 of dark grey area (Cr-rich carbides).

Table 3-12: EDX elemental table of SPS Stellite 6.

\begin{tabular}{|c|c|c|c|c|c|}
\hline \multicolumn{2}{|c|}{ Grey (Co-based solid solution) } & \multicolumn{3}{c|}{ Dark (Cr-rich carbide) } \\
\hline Element & wt.\% & at.\% & Element & wt.\% & at.\% \\
\hline C & 7.67 & 28.59 & C & 24.55 & 58.65 \\
\hline Si & 0.97 & 1.54 & O & 2.51 & 4.50 \\
\hline Cr & 26.80 & 23.09 & Si & 0.87 & 0.89 \\
\hline Fe & 1.37 & 1.10 & $\mathbf{C r}$ & $\mathbf{3 7 . 2 5}$ & $\mathbf{1 8 . 1 3}$ \\
\hline Co & $\mathbf{5 8 . 6 3}$ & $\mathbf{4 4 . 5 7}$ & Fe & 1.07 & 0.55 \\
\hline W & 4.56 & 1.11 & Co & 30.34 & 16.74 \\
\hline \multicolumn{7}{|r|}{} & W & 3.40 & 0.53 \\
\hline
\end{tabular}

\subsubsection{Microstructure of PTA HE6}

The SEM microstructure of PTA HE6 specimen at low magnification (Figure 3-34 a) shows a typical dendritic casting morphology, similar to that of PTA Stellite 6, but it exhibits a unique combination of solid solution, carbides, and eutectic zones. Moreover, the bright area is much more than that in the Stellite 6 specimen due to much higher $\mathrm{W}$ and Mo contents. It is worth 
to note that almost all the W/Mo-rich areas coexist within the lamellar eutectic zone, as shown in Figure 3-34 (b). The EDX results in Figure 3-35 and Table 3-13 confirm that the bright area is W/Mo-rich carbides/intermetallics, the dark area is $\mathrm{Cr} / \mathrm{Fe}$-rich carbides, and the gray area is solid solution, according to the elemental concentrations. However, unlike Co-based solid solution in Stellite 6, the solid solution of HE6 alloy contains equiatomic $\mathrm{Co}, \mathrm{Cr}, \mathrm{Fe}$ and $\mathrm{Ni}$.

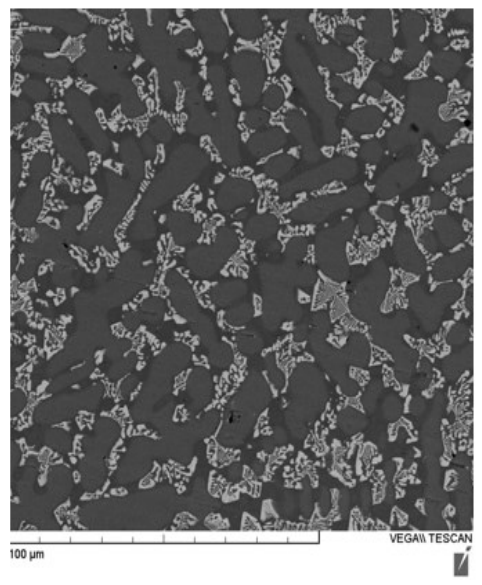

(a)

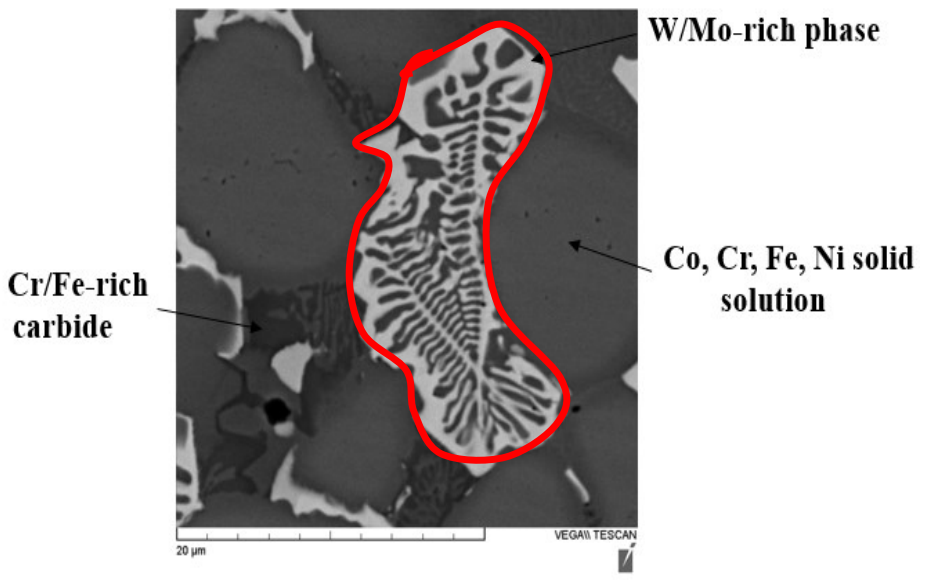

(b)

Figure 3-34: PTA HE6 microstructure (a) at low magnification, (b) at high magnification. 


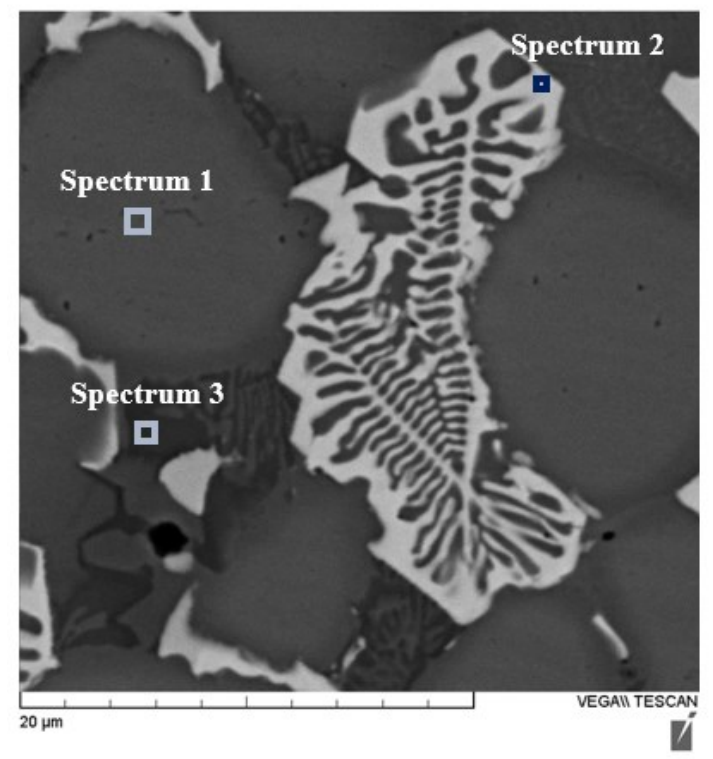

(a)
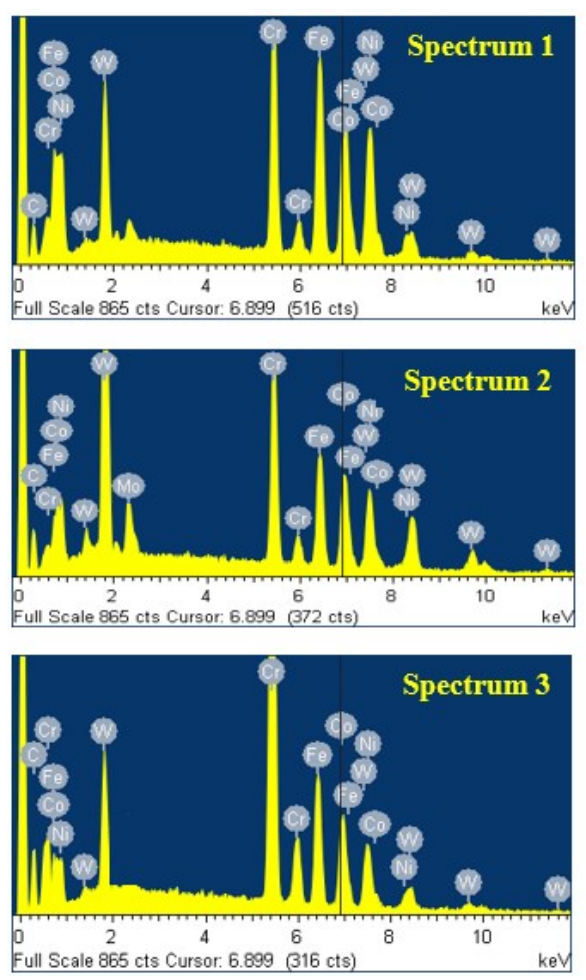

(b)

Figure 3-35: EDX spectra of PTA HE6 microstructure: (a) locations of spectrum, (b) spectrum 1 of grey area (solid solution), spectrum 2 of bright area (W/Mo-rich carbides/intermetallics), spectrum 3 of dark area $(\mathrm{Cr} / \mathrm{Fe}$-rich carbides $)$.

Table 3-13: EDX elemental table of PTA HE6.

\begin{tabular}{|c|c|c|c|c|c|c|c|c|}
\hline \multicolumn{2}{|c|}{ Grey (solid solution) } & \multicolumn{3}{c|}{$\begin{array}{c}\text { Bright (W/Mo-rich } \\
\text { carbide/intermetallic) }\end{array}$} & \multicolumn{3}{c|}{$\begin{array}{c}\text { Dark (Cr/Fe-rich } \\
\text { carbide) }\end{array}$} \\
\hline Element & wt.\% & at.\% & Element & wt.\% & at.\% & Element & wt.\% & at.\% \\
\hline C & 9.32 & 35.32 & C & 11.54 & 45.31 & Cr & $\mathbf{4 0 . 6 1}$ & $\mathbf{4 0 . 5 0}$ \\
\hline Cr & $\mathbf{1 6 . 2 6}$ & $\mathbf{1 4 . 2 3}$ & Cr & 15.00 & 14.92 & Fe & $\mathbf{1 8 . 9 3}$ & $\mathbf{1 6 . 1 8}$ \\
\hline Fe & $\mathbf{2 0 . 1 7}$ & $\mathbf{1 6 . 4 3}$ & Fe & 12.48 & 10.53 & Co & 12.13 & 10.67 \\
\hline Co & $\mathbf{1 8 . 8 3}$ & $\mathbf{1 4 . 5 3}$ & Co & 11.95 & 9.56 & Ni & 10.15 & 8.96 \\
\hline Ni & $\mathbf{2 0 . 3 6}$ & $\mathbf{1 5 . 7 7}$ & Ni & 11.13 & 8.94 & W & 13.59 & 3.83 \\
\hline W & 15.06 & 3.73 & W & $\mathbf{3 4 . 3 1}$ & $\mathbf{8 . 8 0}$ & C & 4.60 & 19.85 \\
\hline \multicolumn{2}{|c|}{} & & Mo & $\mathbf{3 . 5 9}$ & $\mathbf{1 . 9 4}$ & & \multicolumn{3}{|l}{} \\
\hline
\end{tabular}




\subsubsection{Microstructure of SPS HE6 $(45 \mu \mathrm{m})$}

Figure 3-36 shows the microstructure of SPS HE6 $(45 \mu \mathrm{m})$ specimen. Overall, it is similar to a PM microstructure. In addition, it is shown that the original particle boundaries remain in most of the microstructure, as marked by the circles in Figure 3-36 (a). This implies that after the SPS process, the bulk alloy can inherit powder particle features. Also, the grain growth during consolidation might be restrained in the SPS because of the application of a short sintering time, rapid heating, and low-temperature sintering [86]. According to the EDX results (Figure 3-37 and Table 3-14), the bright area is W/Mo-rich phase. Since the dark and gray areas are too small to be distinguished evidently by the EDX machine, the two areas show similar compositions of mainly equiatomic $\mathrm{Co}, \mathrm{Cr}, \mathrm{Fe}$, and $\mathrm{Ni}$. However, combining the $\mathrm{XRD}$ results, it is believed that the existence of carbides contributes to the visual difference in phases of the SPS form. Thus, it is still possible to identify different phases according to the distinct greyscales. In addition, it can be inferred that a series of intermetallics are dispersed over the alloy which may cause inaccurate EDX results. As a result, Figure 3-36 (b) indicates the three groups of phases.

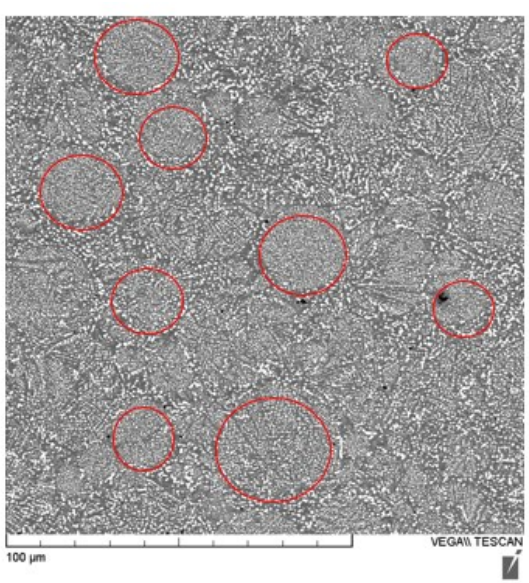

(a)

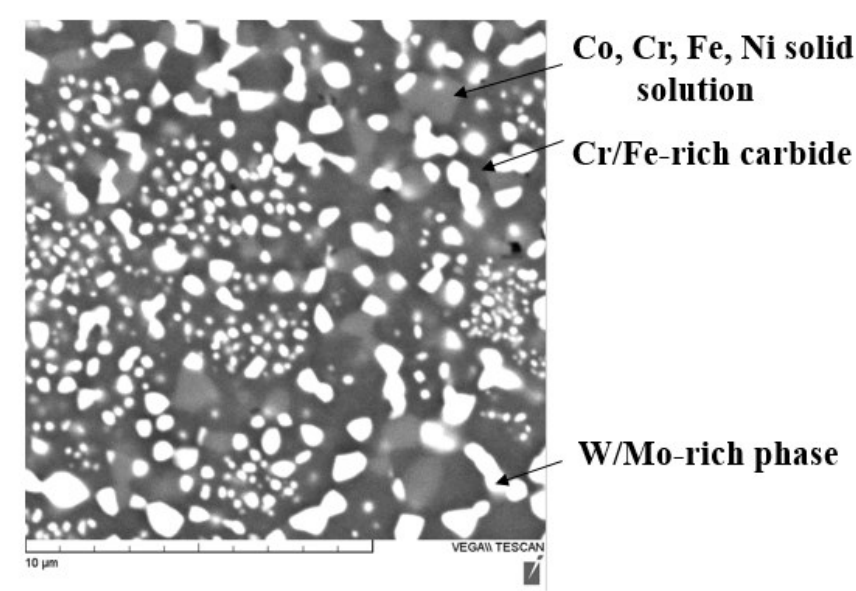

(b)

Figure 3-36: SPS HE6 (45 $\mu \mathrm{m})$ microstructure (a) at low magnification, (b) at high magnification. 


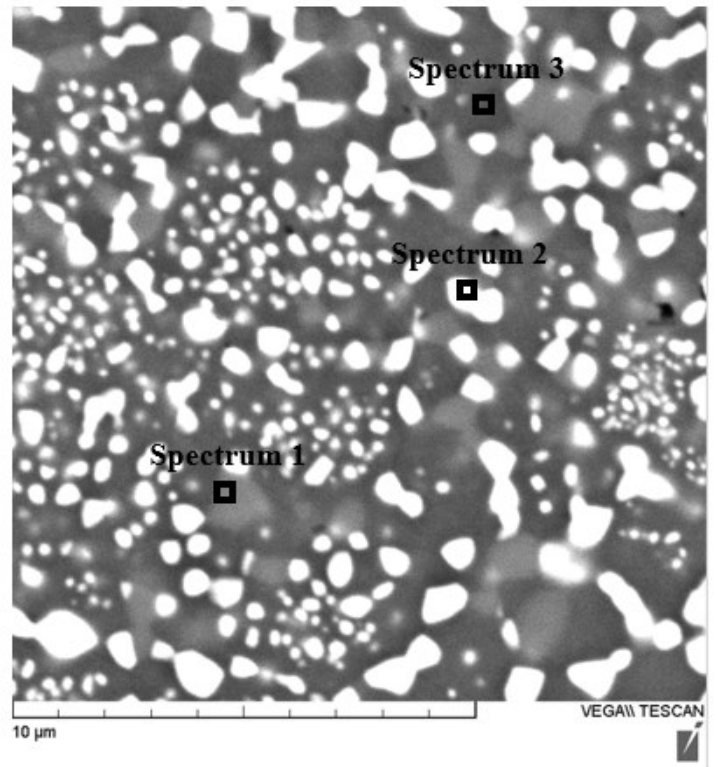

(a)
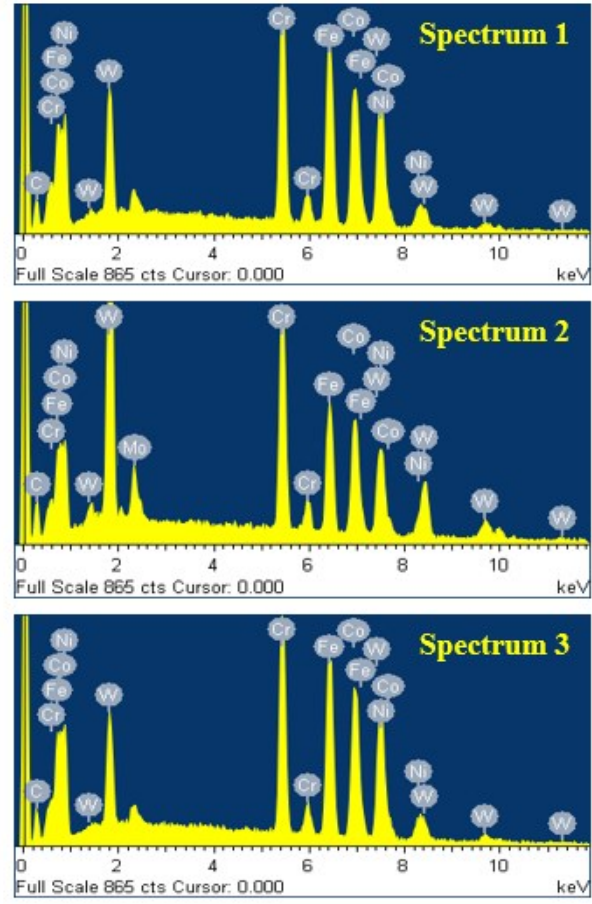

(b)

Figure 3-37: EDX spectra of SPS HE6 $(45 \mu \mathrm{m})$ microstructure: (a) locations of spectrum, (b) spectrum 1 of grey area (solid solution), spectrum 2 of bright area (W/Mo-rich phase), spectrum 3 of dark area (similar to grey area).

Table 3-14: EDX elemental table of SPS HE6 (45 $\mu \mathrm{m})$.

\begin{tabular}{|c|c|c|c|c|c|c|c|c|}
\hline \multicolumn{2}{|c|}{ Grey (solid solution) } & \multicolumn{3}{c|}{$\begin{array}{c}\text { Bright (W/Mo-rich } \\
\text { carbide/intermetallic) }\end{array}$} & \multicolumn{3}{c|}{ Dark (same as Grey) } \\
\hline Element & wt.\% & at.\% & Element & wt.\% & at.\% & Element & wt.\% & at.\% \\
\hline C & 8.76 & 33.23 & C & 25.05 & 68.61 & C & 8.83 & 33.37 \\
\hline Cr & $\mathbf{1 6 . 7 6}$ & $\mathbf{1 4 . 6 9}$ & $\mathbf{C r}$ & 16.78 & 10.62 & Cr & $\mathbf{1 6 . 5 2}$ & $\mathbf{1 4 . 4 2}$ \\
\hline Fe & $\mathbf{2 0 . 2 6}$ & $\mathbf{1 6 . 5 4}$ & Fe & 8.01 & 4.72 & Fe & $\mathbf{2 0 . 4 1}$ & $\mathbf{1 6 . 5 9}$ \\
\hline Co & $\mathbf{1 9 . 9 7}$ & $\mathbf{1 5 . 8 8}$ & $\mathbf{C o}$ & 8.46 & 4.72 & Co & $\mathbf{2 0 . 4 3}$ & $\mathbf{1 5 . 7 4}$ \\
\hline Ni & $\mathbf{2 1 . 1 3}$ & $\mathbf{1 6 . 4}$ & Ni & 7.97 & 4.47 & Ni & $\mathbf{2 1 . 9 4}$ & $\mathbf{1 6 . 9 6}$ \\
\hline W & 13.13 & 3.26 & Mo & $\mathbf{5 . 0 5}$ & $\mathbf{1 . 7 3}$ & W & 11.87 & 2.93 \\
\hline \multicolumn{2}{|r|}{} & & W & $\mathbf{2 8 . 6 8}$ & $\mathbf{5 . 1 3}$ & & & \\
\hline
\end{tabular}




\subsubsection{Microstructure of SPS HE6 $(150 \mu \mathrm{m})$}

The microstructure of SPS HE6 $(150 \mu \mathrm{m})$ specimen is similar to that of SPS HE6 $(45 \mu \mathrm{m})$ specimen, indicating that the size of the initial HE6 alloy powder has little effect on its microstructure evolved during the SPS process, according to the microstructure in Figure 3-38. The only notable difference for particle sizes is larger original particle boundaries of SPS HE6 $(150 \mu \mathrm{m})$ specimen, as marked by the circles in Figure 3-38 (a), which is attributed to the larger size of the original HE6 alloy powder. The combination of the EDX results (Figure 3-39 and Table 3-15) and XRD pattern suggests that the bright area is W/Mo-rich phase, the grey area is multielement (Co-Cr-Fe-Ni) solid solution, and the dark area represents carbides.

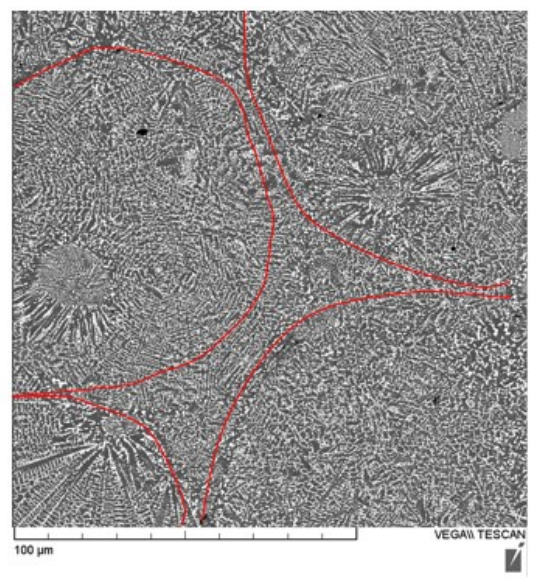

(a)

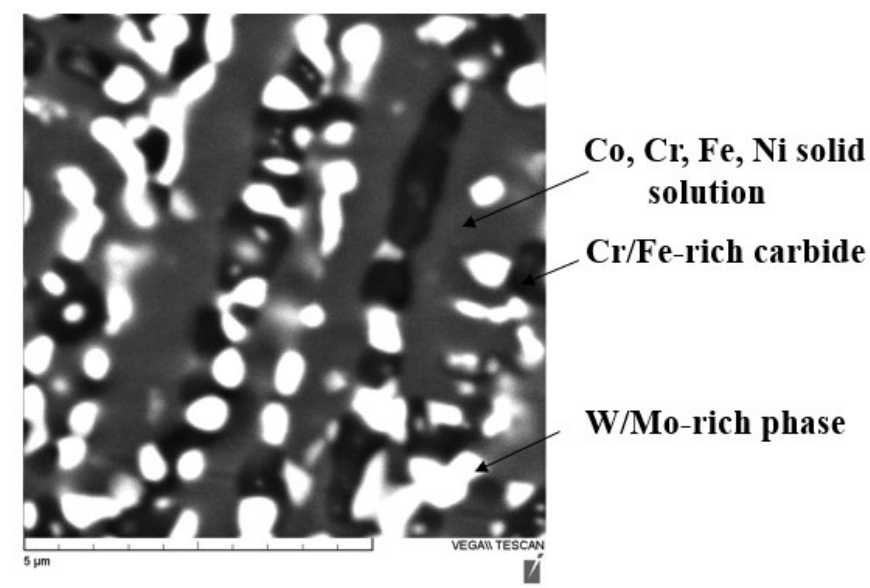

(b)

Figure 3-38: SPS HE6 $(150 \mu \mathrm{m})$ microstructure (a) at low magnification, (b) at high magnification. 


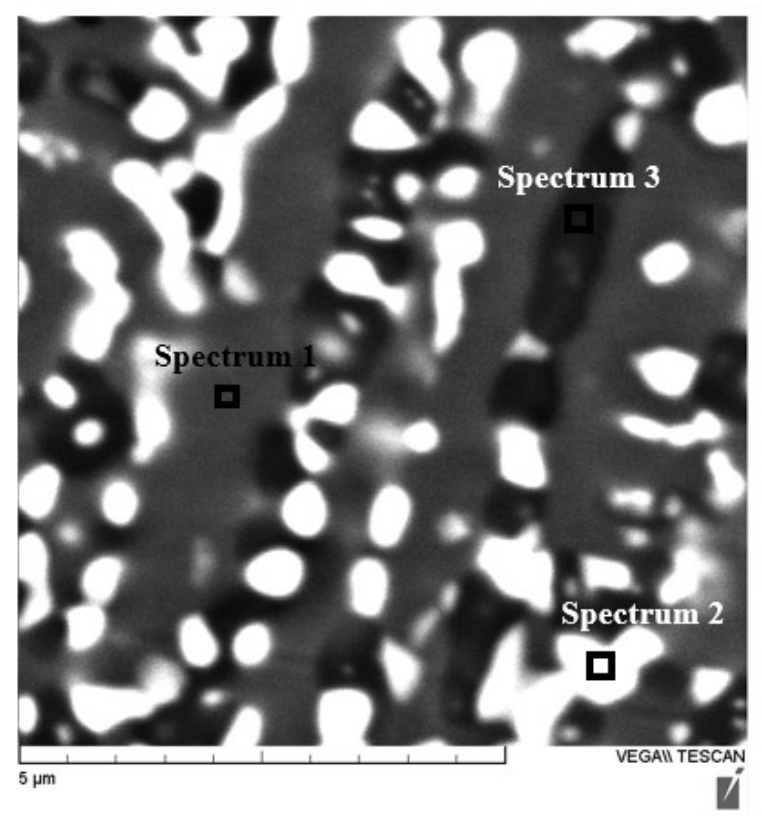

(a)
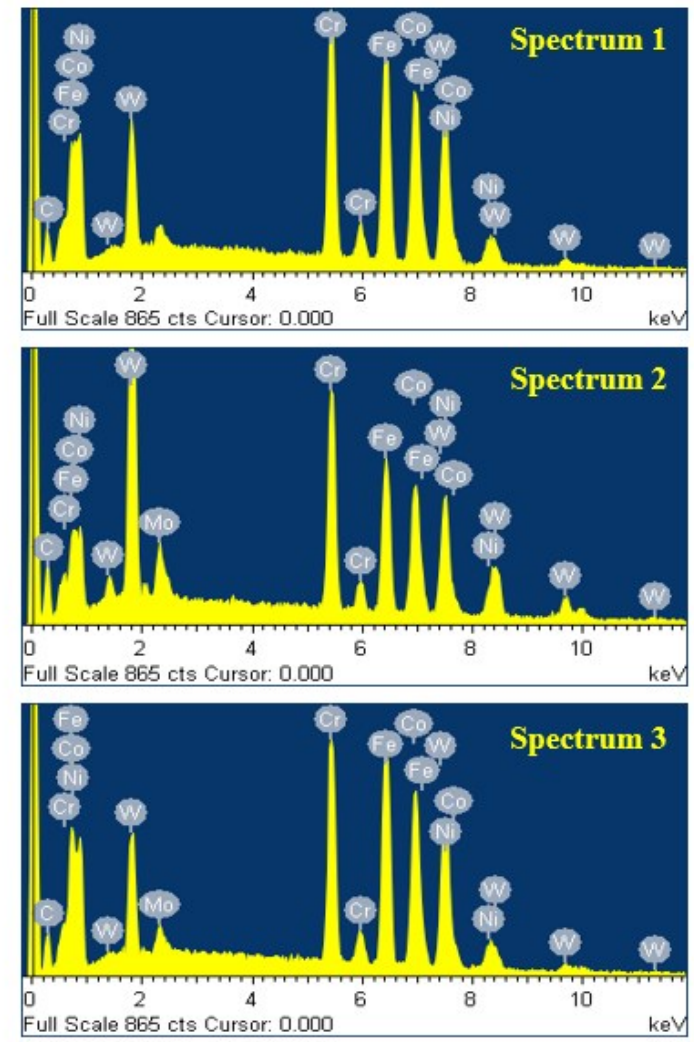

(b)

Figure 3-39: EDX spectra of SPS HE6 $(150 \mu \mathrm{m})$ microstructure: (a) locations of spectrum, (b) spectrum 1 of grey area (solid solution), spectrum 2 of bright area (W/Mo-rich phase), spectrum 3 of dark area (carbides).

Table 3-15: EDX elemental table of SPS HE6 $(150 \mu \mathrm{m})$.

\begin{tabular}{|c|c|c|c|c|c|c|c|c|}
\hline \multicolumn{3}{|c|}{ Grey (solid solution) } & \multicolumn{3}{|c|}{ Bright (W/Mo-rich carbide/intermetallic) } & \multicolumn{3}{|c|}{ Dark (carbide) } \\
\hline Element & wt. $\%$ & at. $\%$ & Element & wt. $\%$ & at. $\%$ & Element & wt.\% & at.\% \\
\hline $\mathrm{C}$ & 8.83 & 33.37 & $\mathrm{C}$ & 29.66 & 74.28 & $\mathrm{C}$ & 30.76 & 69.84 \\
\hline $\mathrm{Cr}$ & 16.52 & 14.42 & $\mathrm{Cr}$ & 11.12 & 6.43 & $\mathrm{Cr}$ & 28.91 & 15.16 \\
\hline $\mathrm{Fe}$ & 20.41 & 16.59 & $\mathbf{F e}$ & 8.11 & 4.37 & $\mathbf{F e}$ & 10.37 & 5.06 \\
\hline Co & 20.43 & 15.74 & Co & 8.55 & 4.36 & Co & 8.86 & 4.10 \\
\hline $\mathbf{N i}$ & 21.94 & 16.96 & $\mathbf{N i}$ & 8.03 & 4.11 & $\mathbf{N i}$ & 7.61 & 3.53 \\
\hline W & 11.87 & 2.93 & Mo & 5.30 & 1.66 & Mo & 2.24 & 0.64 \\
\hline & & & $\mathbf{W}$ & 29.22 & 4.78 & $\mathbf{W}$ & 11.26 & 1.67 \\
\hline
\end{tabular}




\subsubsection{Determination of carbide volume fraction}

In general, the properties of carbon-containing alloys, such as hardness, corrosion and wear resistance, are determined by the carbide volume fraction of the materials. In the present study, the carbide volume fraction of each Stellite 6 and HE6 alloy specimen was determined using the image processing software, ImageJ (Version 5.3.2). Basically, the volume fraction of a phase was calculated by adjusting the threshold of colors to convert the greyscale image to a binary image of the microstructure so that different gray scales can be quantified, respectively. Figure 3-40 to Figure 3-44 show the image conversion results of Stellite 6 and HE6 specimens for determining carbide volume fractions, using the ImageJ software.

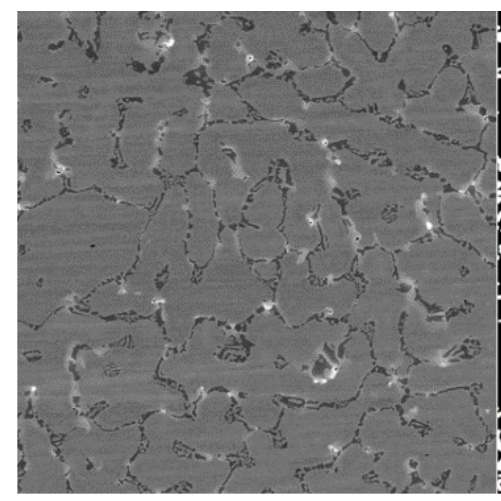

(a)

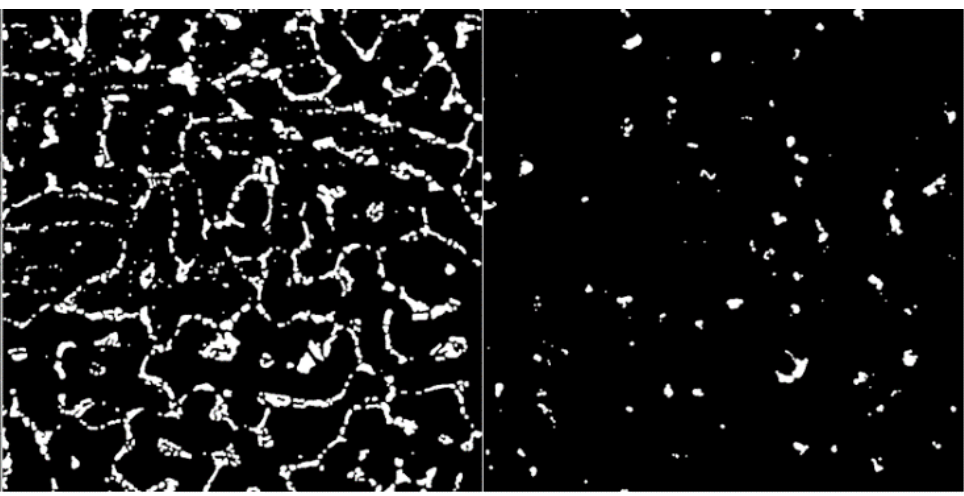

(b) (c)

Figure 3-40: ImageJ processing of PTA Stellite 6 (a) original SEM image, (b) Cr-rich carbides highlighted, (c) W/Mo-rich intermetallics highlighted. 


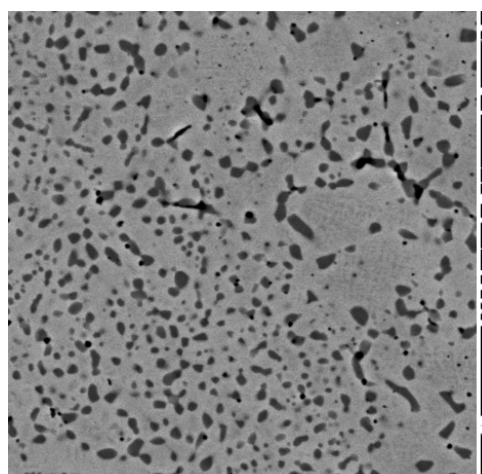

(a)

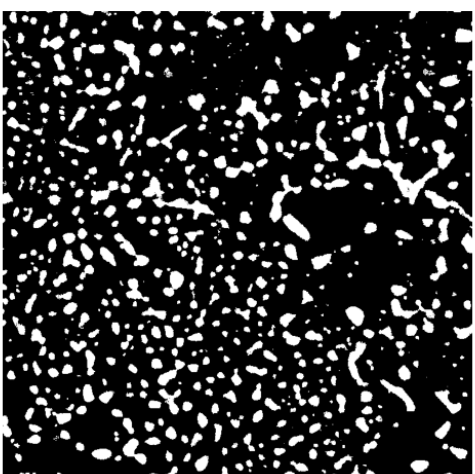

(b)

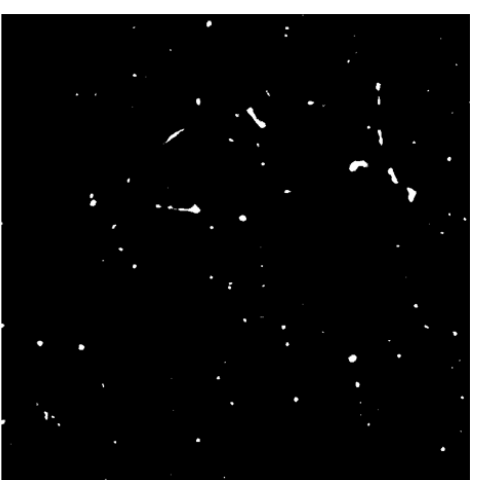

(c)

Figure 3-41: ImageJ processing of SPS Stellite 6 (a) original SEM image, (b) $\mathrm{M}_{23} \mathrm{C}_{6}$ carbides highlighted. (c) $\mathrm{M}_{2} \mathrm{C}$ carbides highlighted.

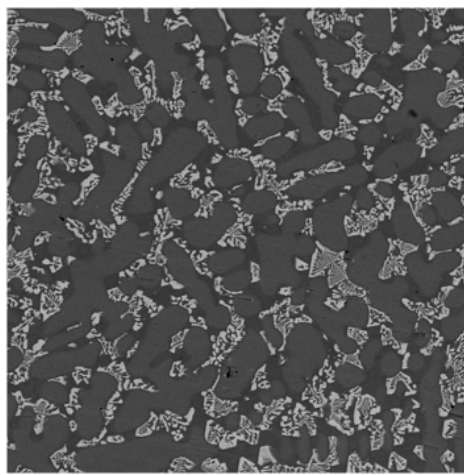

(a)

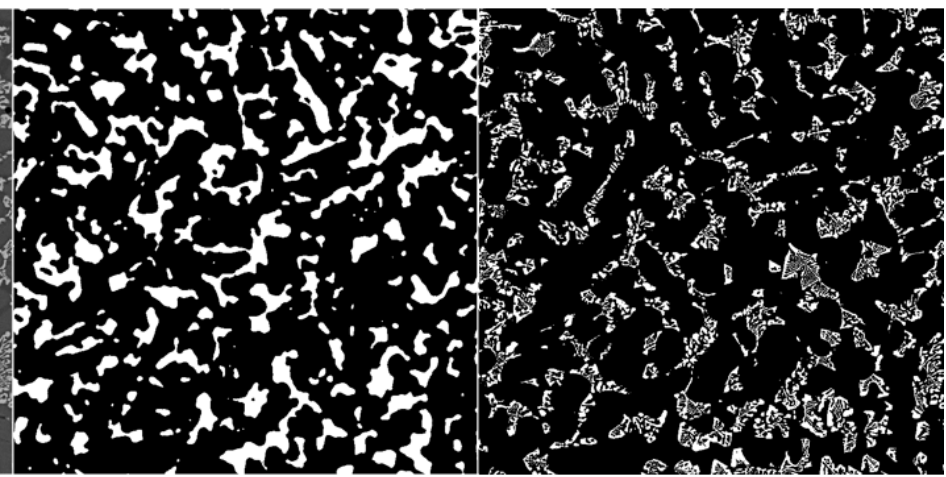

(b) (c)

Figure 3-42: ImageJ processing of PTA HE6 (a) original SEM image, (b) $\mathrm{Cr} / \mathrm{Fe}$-rich carbides highlighted, (c) W/Mo-rich intermetallics/carbides highlighted.

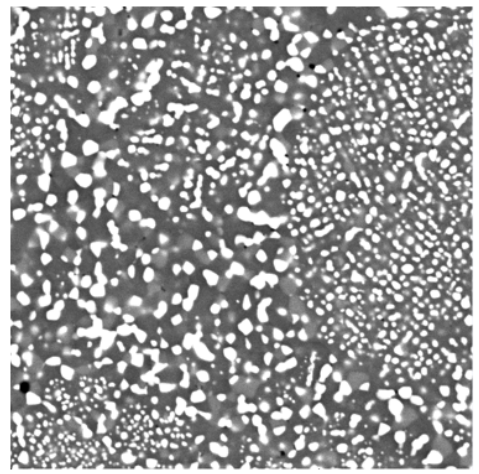

(a)

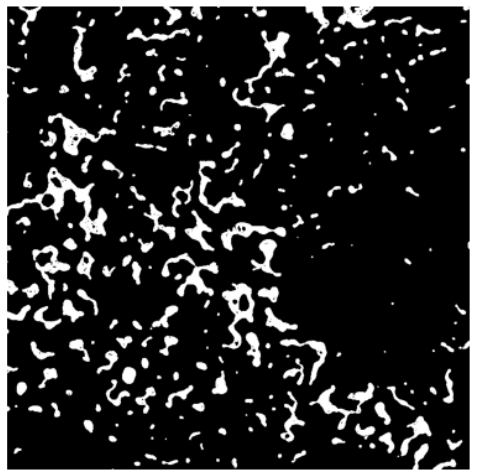

(b)

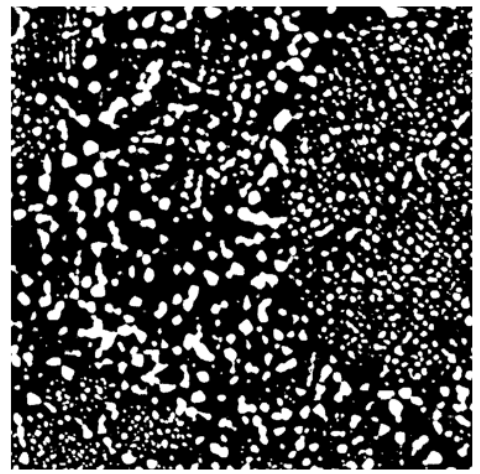

Figure 3-43: ImageJ processing of SPS HE6 (45 $\mu \mathrm{m})$ (a) original SEM image, (b) $\mathrm{Cr} / \mathrm{Fe}-\mathrm{rich}$ carbides highlighted, (c) W/Mo-rich intermetallics/carbides highlighted. 


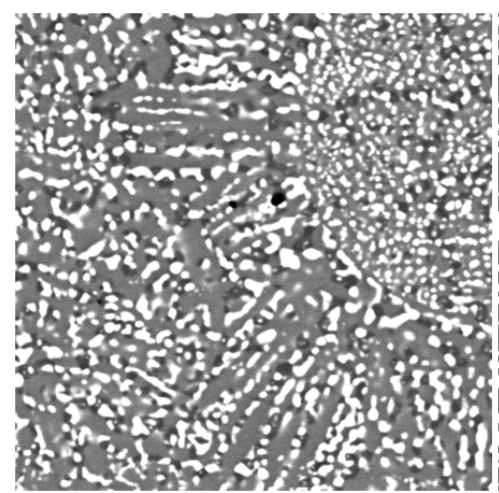

(a)

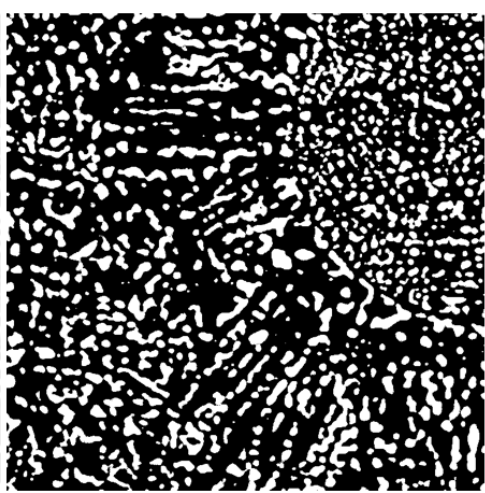

(b)

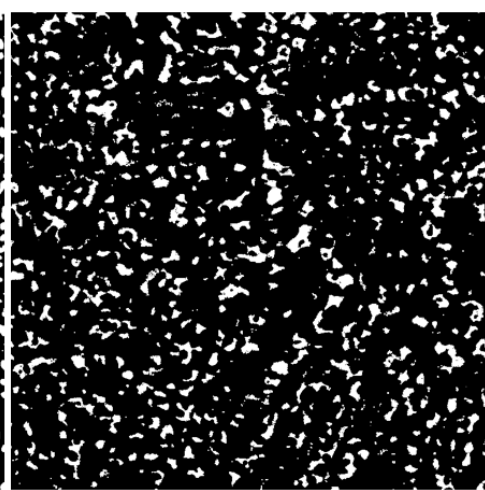

(c)

Figure 3-44: ImageJ processing of SPS HE6 $(150 \mu \mathrm{m})$ (a) original SEM image, (b) W/Mo-rich intermetallics/carbides highlighted, (c) $\mathrm{Cr} / \mathrm{Fe}$-rich carbides highlighted.

The results of carbide volume fraction measurement for Stellite 6 and HE6 alloy specimens are summarized in Table 3-16. For Stellite 6 alloy, the most distinct difference is W/Mo-rich phase, which is found in the PTA specimen but not in the SPS specimen. For HE6 alloy, it is evident that the amount of W/Mo-rich phases in the SPS specimen is much larger than that in the PTA specimen, but $\mathrm{Cr} / \mathrm{Fe}$-rich carbide phases are just the opposite. Between Stellite 6 and HE6 alloy, the latter has a larger volume fraction of overall precipitates (carbides and intermetallics).

Table 3-16: Volume fractions (vol.\%) of precipitates in tested alloys.

\begin{tabular}{|c|c|c|c|c|}
\hline & \multicolumn{2}{|c|}{ Carbides } & $\begin{array}{c}\text { W/Mo-rich } \\
\text { intermetallics/carbides }\end{array}$ & $\begin{array}{c}\text { Overall } \\
\text { precipitates }\end{array}$ \\
\hline PTA-Stellite 6 & \multicolumn{2}{|c|}{17.8} & 3.8 & 21.6 \\
\hline PTA-HE6 & \multicolumn{2}{|c|}{20.8} & 14.2 & 35.0 \\
\hline SPS-Stellite 6 & $17.7\left(\mathrm{M}_{23} \mathrm{C}_{6}\right)$ & $0.6\left(\mathrm{M}_{2} \mathrm{C}\right)$ & $\mathrm{N} / \mathrm{A}$ & 18.3 \\
\hline SPS-HE6-45 & \multicolumn{2}{|c|}{10.2} & 25.8 & 36.0 \\
\hline SPS-HE6-150 & \multicolumn{2}{|c|}{7.3} & 26.8 & 34.1 \\
\hline
\end{tabular}

\subsubsection{Summary of microstructural analyses}

Stellite 6 alloy has been studied extensively in microstructure since it was created. The results of microstructural analysis for this alloy obtained in this study are consistent with previous findings [32][83][87][88]. For the PTA specimen, two types of strengthening agents, which are 
Cr-rich carbides (17.8 vol.\%) and minor W-rich intermetallics (3.8 vol.\%), are precipitated in Co solid solution matrix. However, SPS Stellite 6 specimen is found not containing W-rich intermetallics, but in addition to similar amount of Cr-rich carbides $\left(\mathrm{M}_{23} \mathrm{C}_{6}\right)$, a new type of carbides $\left(\mathrm{M}_{2} \mathrm{C}\right.$, where $\mathrm{M}$ represents $\mathrm{W}$ and $\left.\mathrm{Fe}\right)$ in a very small amount $(0.6$ vol.\%) is precipitated in this specimen. Regarding the microstructure morphologies, PTA Stellite 6 displays typical characteristics of casting microstructure of this alloy in that the carbides form a semi-continuous network in the solid solution matrix. As for SPS Stellite 6, it has a common microstructure of PM processed Stellite alloys, in which fine carbides are distributed uniformly over the solid solution matrix. From the microstructural analyses of the Stellite 6 specimens, it is evident that PTA and SPS manufacturing processes not only result in distinct microstructure morphologies but also alter phase constituents of the alloy. This is because the former is the liquidus processing method while the latter is the liquidus/solidus processing method; the two techniques manufacture materials in different mechanisms. In the PTA process, Stellite 6 powder was entirely melted so that the phase transformations occurred during the solidification of the molten powder. However, in the SPS process, since the powder particles were only partially melted, the main mechanism of phase formation was elemental diffusion. Therefore, the SPS specimen retained some microstructure features of the Stellite 6 powder.

HE6 alloy is a new alloy, which was first studied in this research. Owing to the unique chemical composition, which combines the concepts of both Stellite alloy and HEA, HE6 alloy has a novel microstructure that takes the main features of Stellite alloys but also exhibits the characteristics of HEAs. First, due to the presence of carbon ( $0.96 \mathrm{wt} . \%)$, large amounts of carbides ( $\mathrm{Cr} / \mathrm{Fe}-$ rich and W/Mo-rich) were precipitated in the solid solution matrix. Second, the solid solution is not Co-based, which differs from Stellite alloys, due to near equiatomic $\mathrm{Co}-\mathrm{Cr}-\mathrm{Fe}-\mathrm{Ni}$ 
composition. Instead, the FCC solid solution consists of Co, Cr, Fe and Ni. Third, the presence of W (19 wt.\%) promoted the formation of W/Mo-rich intermetallics in large quantities. Although the PTA and SPS processes rendered distinct microstructure morphologies of HE6 alloy, the phase constituents of the specimens from these two processes are the same, only varying in ratio. For example, the W/Mo-rich intermetallics in SPS HE6 specimen are apparently increased, compared with the PTA one, while the $\mathrm{Cr} / \mathrm{Fe}$-rich carbides are reduced. The powder particle size of HE6 alloy had little influence on the microstructure of the SPS specimen.

Combining the results of XRD and SEM/EDX, the composition of each visually different phase can be confirmed, as summarized in

\section{Table 3-17 to}

Table 3-21. For Stellite 6, single FCC Co forms the solid solution of the alloying powder, but HCP Co was generated during the PTA and SPS processes and co-existed with FCC Co in PTA and SPS Stellite 6 specimens. For carbides, the PTA and SPS processes retained the same type of carbide (Cr-rich $\left.\mathrm{M}_{23} \mathrm{C}_{6}\right)$ from the Stellite 6 powder in the bulk specimens. Additionally, the SPS process also induced $\mathrm{M}_{2} \mathrm{C}$ type carbides (Fe/W-rich) with W/Mo-rich intermetallics diminishing in SPS Stellite 6 specimen.

Table 3-17: Identified phases in Stellite 6 powder.

\begin{tabular}{|c|c|c|c|}
\hline Solid solution & Carbides & Other major phases & Other minor phases \\
\hline Co-based & Cr-rich & Intermetallics & Intermetallics \\
\hline \multirow{2}{*}{ FCC Co } & $\mathrm{M}_{23} \mathrm{C}_{6}$ & $\mathrm{Cr}_{4} \mathrm{Ni}_{15} \mathrm{~W}$ & $\mathrm{FeNi}_{3}$ \\
\cline { 3 - 4 } & & $\mathrm{Co}_{3} \mathrm{Mo}$ & $\mathrm{Cr}_{0.095} \mathrm{Fe}_{0.38} \mathrm{Co}_{0.528}$ \\
\hline
\end{tabular}


Table 3-18: Identified phases in PTA Stellite 6.

\begin{tabular}{|c|c|c|c|}
\hline $\begin{array}{c}\text { Solid solution } \\
\text { (grey) }\end{array}$ & $\begin{array}{c}\text { Carbides } \\
\text { (black) }\end{array}$ & $\begin{array}{c}\text { Other major phases } \\
\text { (bright) }\end{array}$ & $\begin{array}{c}\text { Other minor } \\
\text { phases }\end{array}$ \\
\hline Co-based & Cr-rich & Intermetallics & Intermetallics \\
\hline \multirow{3}{*}{$\begin{array}{c}\mathrm{FCC} \mathrm{Co} \\
\mathrm{HCP} \mathrm{Co}\end{array}$} & \multirow{2}{*}{$\mathrm{M}_{23} \mathrm{C}_{6}$} & $\mathrm{Cr}_{4} \mathrm{Ni}_{15} \mathrm{~W}$ & $\mathrm{CrFeCo}$ \\
\cline { 3 - 4 } & & $\mathrm{Co}_{3} \mathrm{Mo}$ & $\mathrm{Cr}_{0.4} \mathrm{Co}_{0.245} \mathrm{Ni}_{0.351}$ \\
\cline { 3 - 4 } & & $\mathrm{Co}_{3} \mathrm{~W}$ & $\mathrm{CrFeCoNi}$ \\
\cline { 2 - 4 } & $\mathrm{Co}_{0.87} \mathrm{~W}_{0.13}$ & $\mathrm{Cr}_{3} \mathrm{Fe}$ \\
\cline { 2 - 4 } & & $\mathrm{Cr}_{0.32} \mathrm{Mo}_{0.04} \mathrm{Co}_{0.64}$ \\
\hline
\end{tabular}

Table 3-19: Identified phases in SPS Stellite 6.

\begin{tabular}{|c|c|}
\hline Solid solution (grey) & Carbides (dark grey, black) \\
\hline Co-based & Cr-rich \\
\hline FCC Co & $\mathrm{M}_{23} \mathrm{C}_{6}(\mathrm{Cr}$-rich, dark grey $)$ \\
\hline $\mathrm{HCP} \mathrm{Co}$ & $\mathrm{M}_{2} \mathrm{C}\left(\mathrm{Fe}_{2} \mathrm{C} / \mathrm{W}_{2} \mathrm{C}\right.$, black $)$ \\
\hline
\end{tabular}

For HE6 alloy, the phase constituents of bulk specimens are much more complex than that of powder form, which means that both the PTA and SPS processes promoted new phases formed in this alloy. On the other hand, near equiatomic $\mathrm{Co}-\mathrm{Cr}-\mathrm{Fe}-\mathrm{Ni}$ composition of HE6 alloy provided more options for phase formation in this alloy. Multi-element FCC solid solution remained stable in bulk HE6 alloy specimens, but there were slight changes in carbides. $\mathrm{M}_{23} \mathrm{C}_{6}$ and $\mathrm{M}_{16} \mathrm{C}$ were retained in the bulk specimens from the HE6 powder, but MC from the powder transferred to $\mathrm{M}_{6} \mathrm{C}$ in bulk HE6. Meanwhile, a series of intermetallics were precipitated in HE6 during the PTA and SPS processes, which were more diverse than that in Stellite 6. The mechanisms behind various carbide and intermetallic formations in HE6 alloy are not yet clear. However, since this alloy contains high $\mathrm{Ni}(20$ wt.\%) which is active in alloy systems, there are more intermetallic compounds containing Ni formed in the alloy matrix.

The phase analyses with XRD show the same phase constituents in the PTA specimen and SPS specimens from two different size powders of HE6 alloy, which suggests that the phases 
formed in HE6 alloy are more dependent on its chemical composition rather than manufacturing method, and original powder size has little impact on the microstructure evolution.

Table 3-20: Identified phases in HE6 powder.

\begin{tabular}{|c|c|c|c|}
\hline Solid solution & Carbides & $\begin{array}{c}\text { Other major } \\
\text { phases }\end{array}$ & Other minor phases \\
\hline $\begin{array}{c}\mathrm{Co}, \mathrm{Cr}, \mathrm{Fe}, \mathrm{Ni} \text { solid } \\
\text { solution }\end{array}$ & $\mathrm{Cr} / \mathrm{Fe}-\mathrm{rich}$ & Intermetallics & Intermetallics \\
\hline $\mathrm{Co}$ & $\mathrm{M}_{23} \mathrm{C}_{6}$ & $\mathrm{WNi}_{4}$ & $\mathrm{Ni}-\mathrm{Cr}-\mathrm{Co}-\mathrm{Mo}$ \\
\hline $\mathrm{Cr}$ & $\mathrm{MC}$ & $\mathrm{MoNi}_{4}$ & $\mathrm{Fe}_{0.66} \mathrm{Ni}_{0.34}$ \\
\hline $\mathrm{Fe}$ & $\mathrm{M}_{16} \mathrm{C}$ & $\mathrm{Cr}_{0.5} \mathrm{Mo}_{0.5}$ & $\mathrm{Cr}_{0.44} \mathrm{~W}_{0.03} \mathrm{Ni}_{0.53}$ \\
\hline & & & $\mathrm{Cr}_{1.93} \mathrm{Fe}_{0.61} \mathrm{Co}_{0.17} \mathrm{Ni}_{5.96} \mathrm{Mo}_{1.15} \mathrm{~W}_{0.18}$ \\
\hline
\end{tabular}

Table 3-21: Identified phases in bulk HE6.

\begin{tabular}{|c|c|c|c|}
\hline Solid solution (grey) & $\begin{array}{c}\text { Carbides } \\
\text { (black) }\end{array}$ & $\begin{array}{c}\text { Other major phases } \\
\text { (bright) }\end{array}$ & $\begin{array}{c}\text { Other minor } \\
\text { phases }\end{array}$ \\
\hline $\begin{array}{c}\mathrm{Co}, \mathrm{Cr}, \mathrm{Fe}, \mathrm{Ni} \text { solid } \\
\text { solution }\end{array}$ & $\mathrm{Cr} / \mathrm{Fe}-$-rich & Intermetallics/carbides & Intermetallics \\
\hline $\mathrm{Co}$ & $\mathrm{M}_{23} \mathrm{C}_{6}$ & $\mathrm{WNi}_{4}$ & $\mathrm{Cr}_{0.5} \mathrm{Ni}_{0.5}$ \\
\hline $\mathrm{Cr}$ & $\mathrm{M}_{16} \mathrm{C}$ & $\mathrm{W}_{1.8} \mathrm{Ni}_{8.1}$ & $\mathrm{Ni}-\mathrm{Cr}-\mathrm{Co}-\mathrm{Mo}$ \\
\hline $\mathrm{Fe}$ & & $\mathrm{Co}_{3} \mathrm{Mo}$ & $\mathrm{Fe}_{0.66} \mathrm{Ni}_{0.34}$ \\
\hline & & $\mathrm{M}_{6} \mathrm{C}(\mathrm{W} / \mathrm{Mo})$ & $\mathrm{Cr}-\mathrm{Ni}-\mathrm{Fe}-\mathrm{C}$ \\
\hline & & & $\mathrm{Ni}-\mathrm{Cr}-\mathrm{Co}-\mathrm{Mo}$ \\
\hline
\end{tabular}




\section{Chapter 4: Performance Evaluation of Bulk Alloys}

\subsection{Hardness Test}

Hardness is one of the critical scales in material characterization. It is a measurement of the resistance of a solid material to shape change occurring when a force is applied to it. This is an essential feature of material selection since many other material properties, such as ductility, elasticity, strain, toughness and strength, are related to it.

\subsubsection{Experimental procedure}

In this research, both Vickers Hardness (HV) and Rockwell Hardness C-Scale (HRC) of bulk specimens of Stellite 6 and HE6 were measured, as a part of performance evaluation of the new HEA alloy. A CLEMEX microhardness tester, model SMT-X7 Dual Indenter, shown in Figure 4-1, was employed to investigate the Vickers hardness of the alloy specimens at room temperature. This microhardness tester system is fully-automated with a computer-controlled workbench, using Vickers indenter standards. The system applies a constant force to a sharp indenter in order to form a permanent indentation on the sample surface. It has a wide load range, from 1 gram force (gf) to $2000 \mathrm{gf}$. The loading duration varies between $5 \sim 60$ seconds. Using a microscope coupled with a digital camera, each phase in the microstructure can be identified and selected for measurement. Meanwhile, the value of hardness is automatically calculated by the built-in software package based on the surface residual indentation area and the applied force. Following the ASTM E384-17 standard [89], the testing parameters used in this research are set, as summarized in Table 4-1. For Vickers hardness, the results were calculated based on measuring the lengths of diagonals of the indentation, as illustrated in Figure 4-2. 


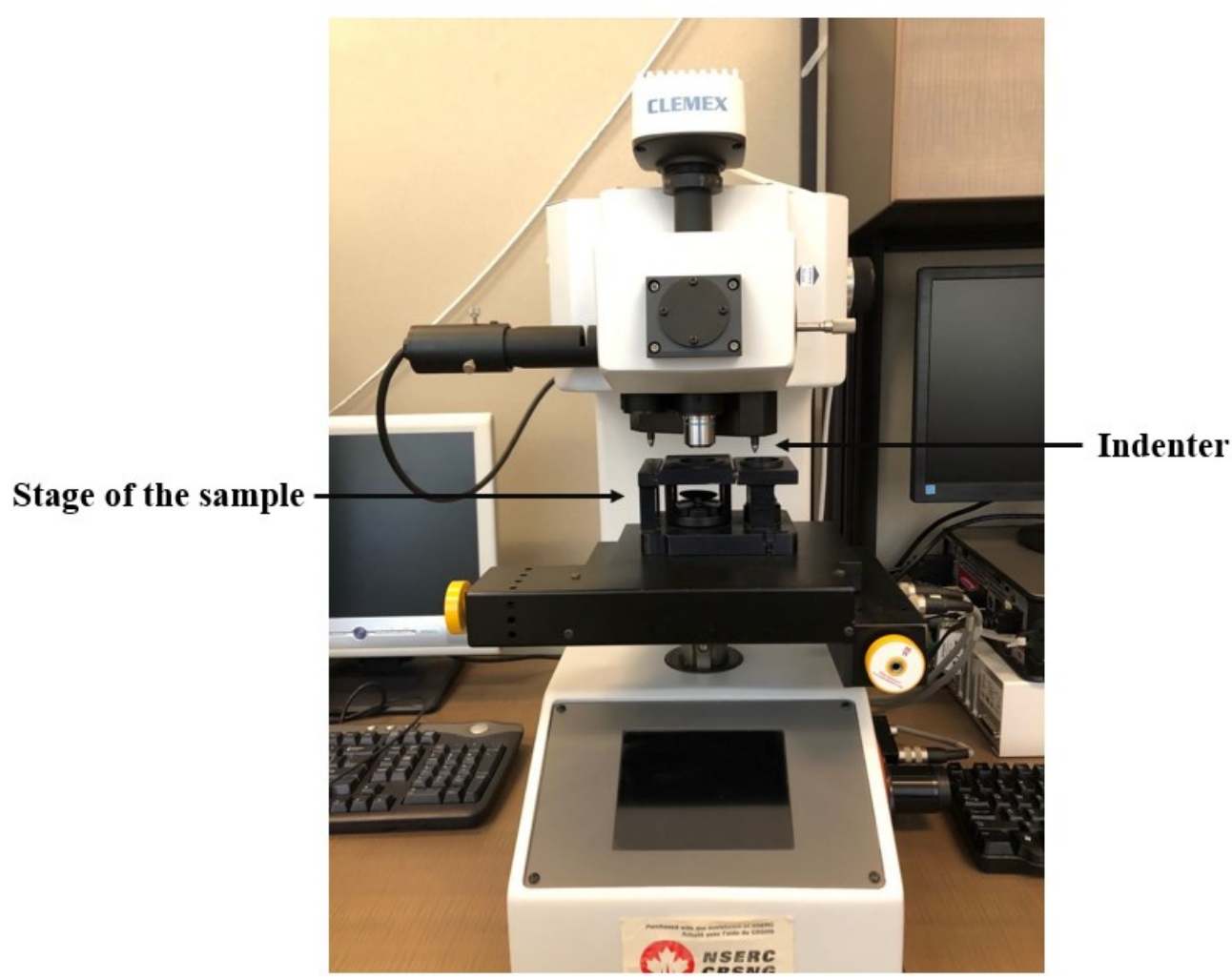

Figure 4-1: CLEMEX Microhardness tester, model SMT-X7 Dual Indenter.

Table 4-1: A summary of hardness test specifications

\begin{tabular}{|c|c|}
\hline Sample surface finish & $\mathrm{Up}$ to $0.06 \mu \mathrm{m}$ final polish \\
\hline Environment & Room temperature \\
\hline Indentation force & $500 \mathrm{gf}$ \\
\hline Indenter dwell time & 10 seconds \\
\hline
\end{tabular}

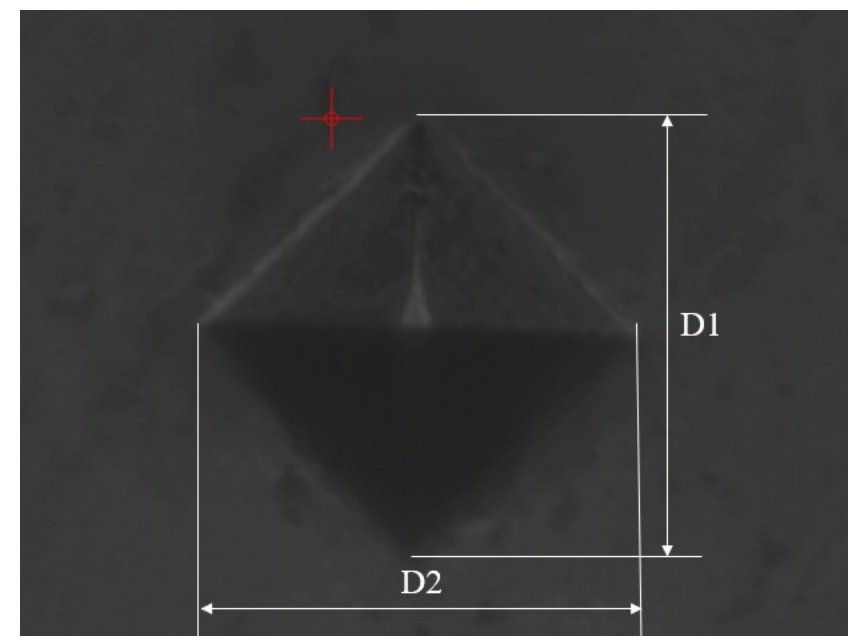

Figure 4-2: Diagonal lengths of an indentation. 
The measurements of HRC hardness of the bulk alloy specimens were conducted on a portable Rockwell Hardness Tester, as shown in Figure 4-3.

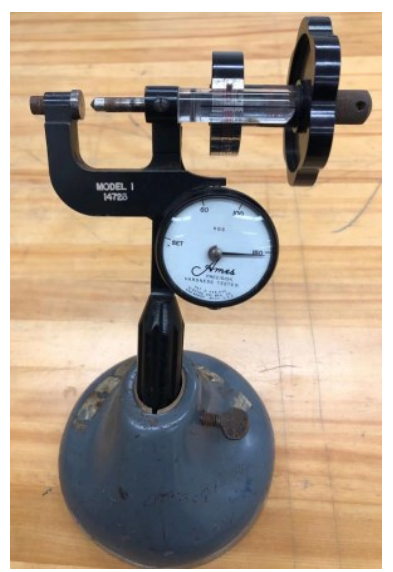

Figure 4-3: A portable Rockwell Hardness tester.

\subsubsection{Hardness results}

To confirm the repeatability of hardness testing results, about 10 measurements were made on each alloy specimen. The average hardness results are illustrated in Figure 4-4. Comparing different manufacturing processes, the hardness of SPS Stellite 6 is $26.3 \mathrm{HV}$ higher than PTA one, while HE6 (45 $\mu \mathrm{m})$ specimens are very similar in hardness. Cross-comparing the two alloy grades, Stellite 6 from both PTA and SPS process is harder than HE6. In terms of powder size, HE6 specimen $(150 \mu \mathrm{m})$ exhibits a slightly higher hardness than HE6 specimen $(45 \mu \mathrm{m})$ in the SPS group. 


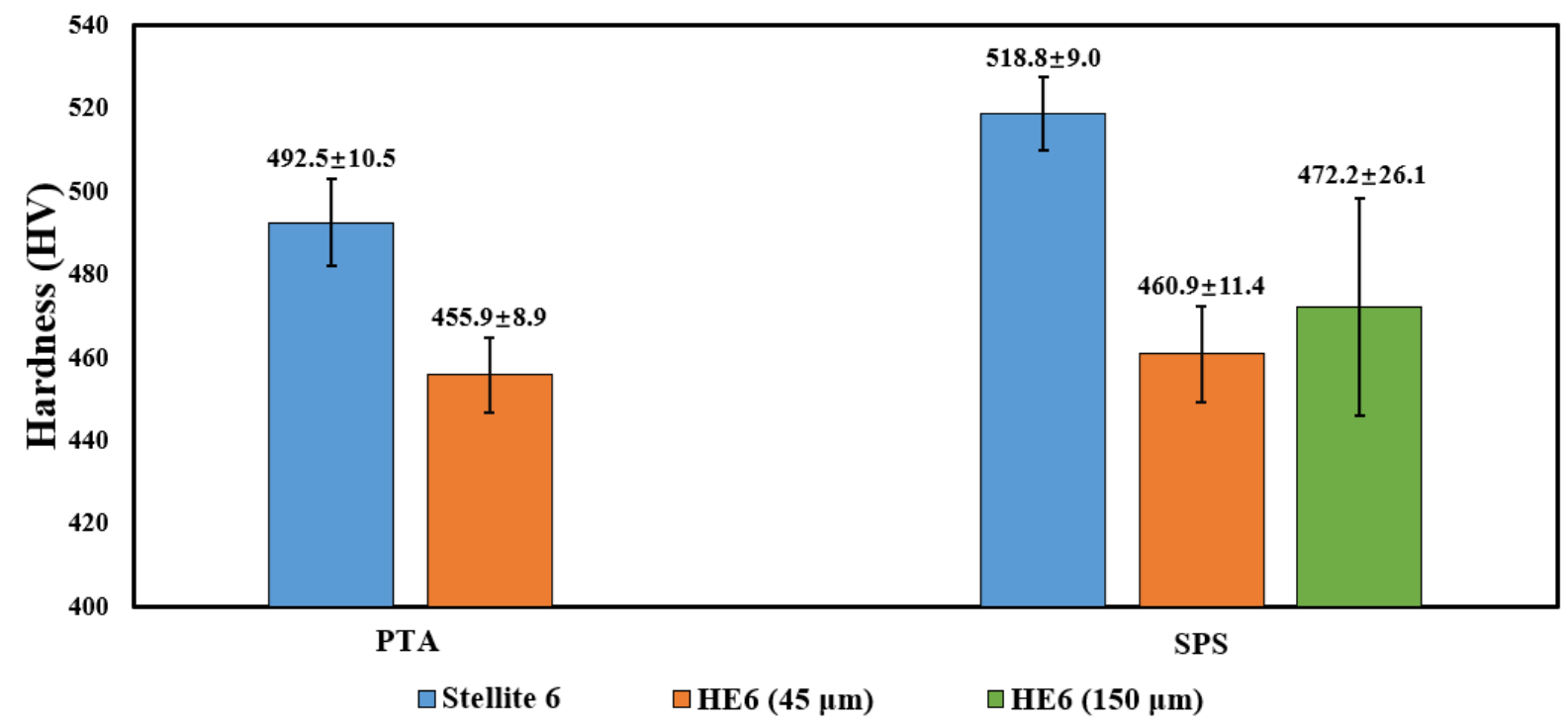

Figure 4-4: Vickers Hardness test results of bulk alloy specimens.

The results of Rockwell Hardness test are presented in Figure 4-5, which comply with the Vickers hardness test results. From both micro and macro hardness tests, it is noticed that, for Stellite 6 alloy, the specimen processed via SPS has evidently higher hardness than the PTA specimen. This may be attributed to the precipitation of $\mathrm{W}_{2} \mathrm{C}$ carbide in the microstructure during the SPS process.

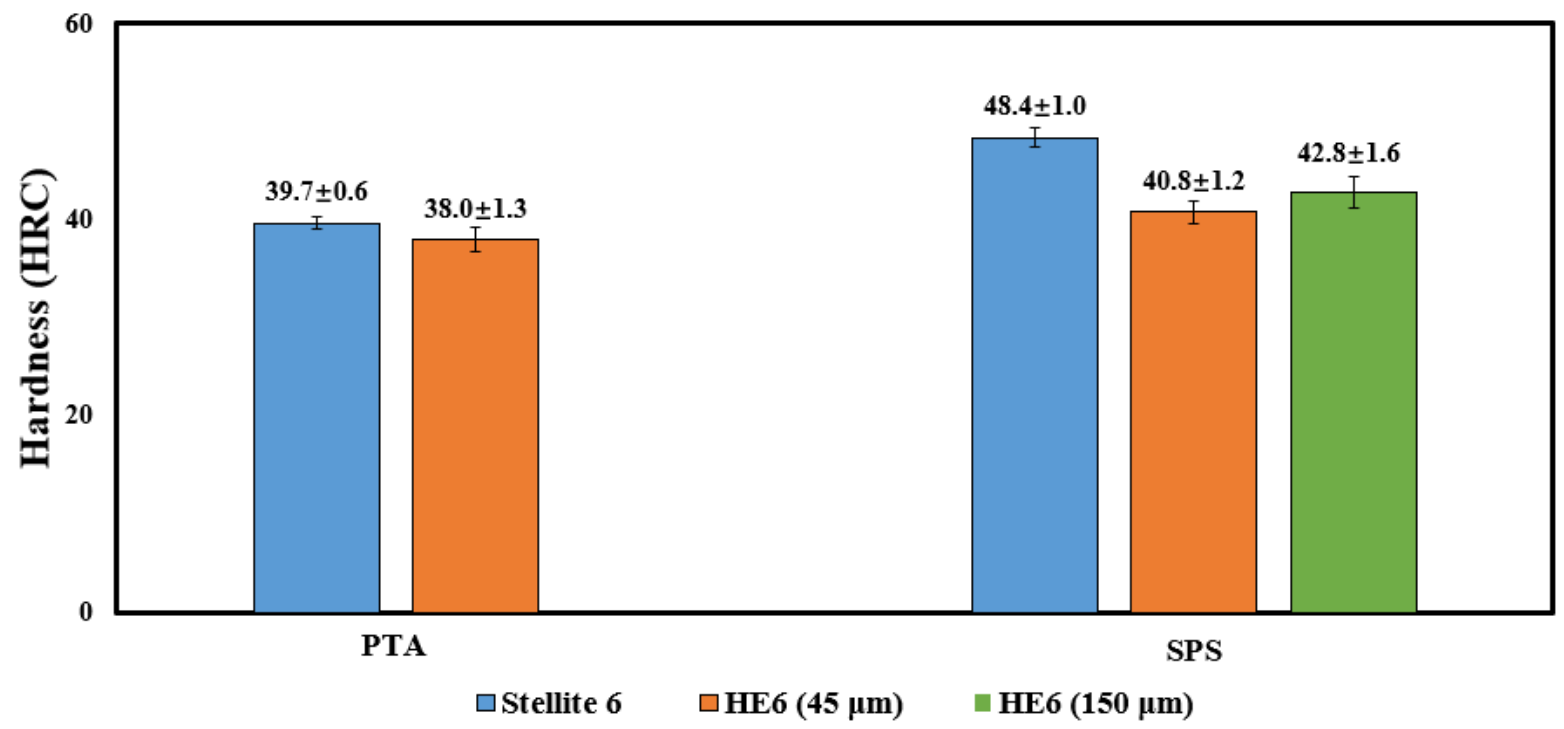

Figure 4-5: Rockwell Hardness test results of bulk alloy specimens. 


\subsection{Wear Test}

\subsubsection{Dry-sliding wear testing facilities}

Dry-sliding wear test method was used to investigate the wear behavior of Stellite 6 and HE6 bulk material. The pin-on-disc wear test following ASTM G99 - 17 Standard [62] was chosen to evaluate the wear resistance and friction coefficient of the bulk alloy specimens.

The wear test was executed using a NEO-TRIBO model MPW110 pin-on-disc wear test system, as shown in Figure 4-6 (a). This method involves a ball-shaped pin mounted on an upper cylindrical holder that revolves around the center, which results in sliding wear occurring on the lower disc-shaped specimen. Therefore, the ball and disc form a friction pair. Figure 4-6 (b) illustrates the friction pair positioning. A fixed axial load is applied to the specimen. A counterweight is applied vertically and attained through a 1:10 lever. A force sensor is equipped to record the friction force at the load-cell in real time, and later it can be used to calculate the friction coefficient of the specimen surface. All the alloy specimens were evaluated at room temperature in dry sliding (unlubricated) mode.

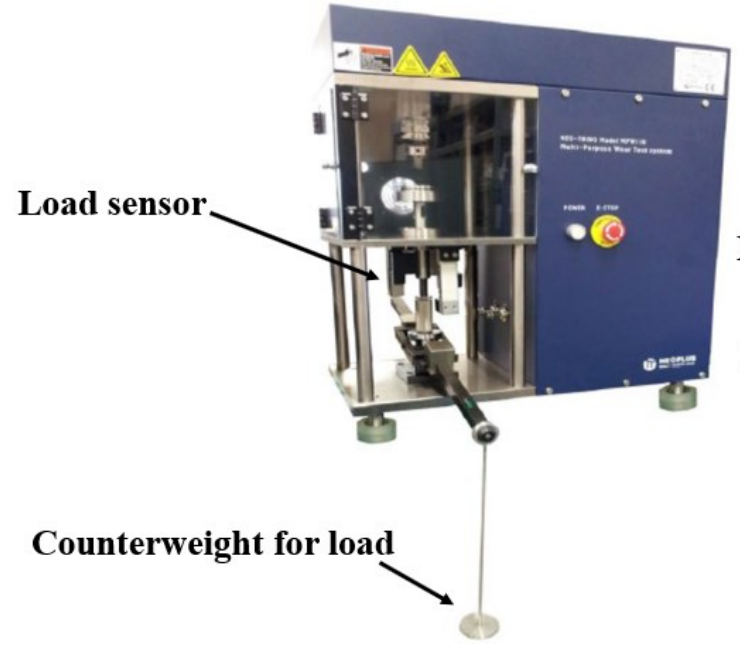

(a)

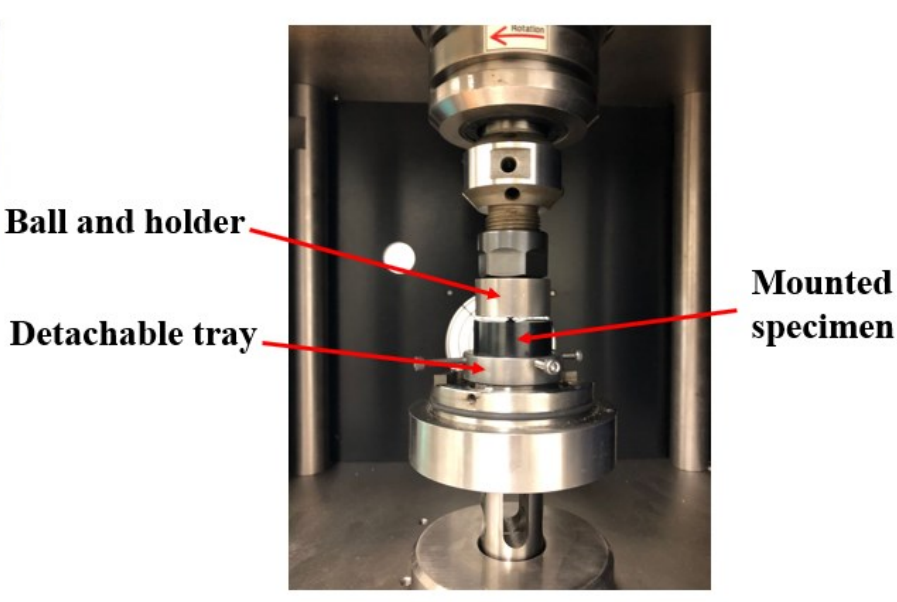

(b)

Figure 4-6: Pin-on-disc tribometer: (a) system assembly, (b) detailed specimen positioning. 
A schematic drawing of the working principle of the pin-on-disc machine is shown in Figure 4-7 [90]. The real-time friction force at load-cell $\left(F_{L}\right)$ is monitored and recorded by the machine software automatically. Hence, using Equation 4.1 to 4.3 , the result of the friction coefficient on the specimen surface can be obtained.

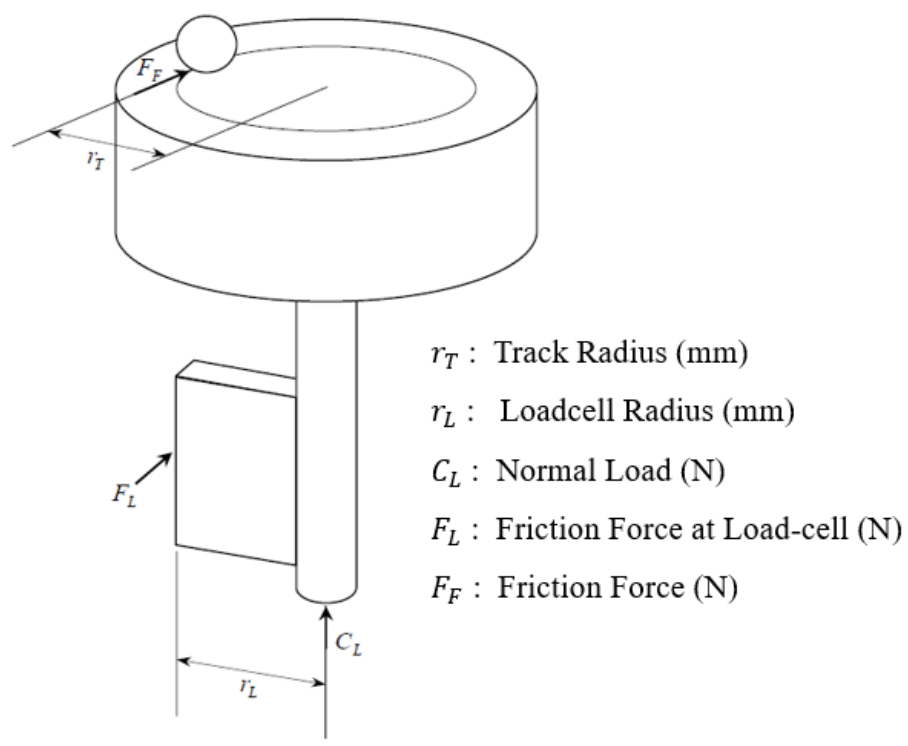

Figure 4-7: Load sensor setup.

$$
\begin{gathered}
r_{T} \times F_{F}=r_{L} \times F_{L} \\
\mu=\frac{F_{F}}{C_{L}} \\
\mu=\frac{F_{L} \times r_{L}}{C_{L} \times r_{T}}
\end{gathered}
$$

The pin used in this research was a spherical $5 \mathrm{~mm}$ diameter ball made of silicon nitride $\left(\mathrm{Si}_{3} \mathrm{~N}_{4}\right)$ with a hardness of $1800 \mathrm{HV}$. Because of the much higher hardness of the ball than the specimen materials (Stellite 6 and HE6), only the wear of the disc specimen was considered in the 
friction pair. The mounted specimen was placed on a detachable tray and fixed to the wear device with screws. As a result of sliding, a wear track was eventually generated on the surface of the disc, as shown in Figure 4-8.

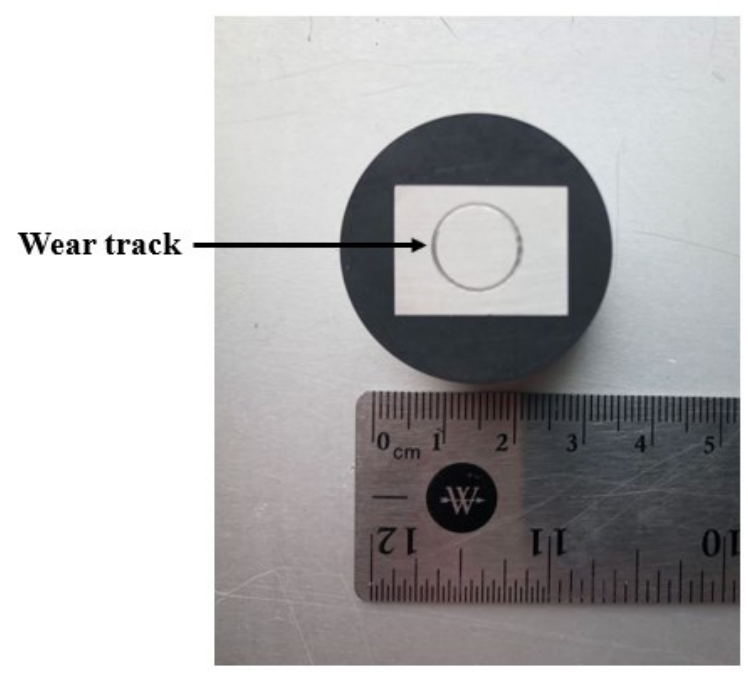

Figure 4-8: A mounted disc specimen after sliding wear.

\subsubsection{Wear test parameters}

For the wear testing, the specimen surface was prepared by polishing with sandpaper up to 400 grit followed by 15 minutes of ultrasonic cleaning to achieve a roughness of $0.8 \mu \mathrm{m}$ arithmetic average or less, according to the ASTM standard G99 - 17 [62]. To investigate the wear behavior of the bulk alloys, several variables need to be selected, such as time/duration, applied force load, and rotational speed, etc. After reviewing previous research about Stellite alloys [23], [65], [91][93], the test parameters for this research were selected as given in Table 4-2. Three repeated tests were conducted to confirm the reputability on each specimen, and then an average wear volume loss was taken for each alloy specimen. 
Table 4-2: A summary of wear test parameters.

\begin{tabular}{|c|c|}
\hline Parameter & \multicolumn{1}{|c|}{ Value } \\
\hline Test duration/time & \multicolumn{1}{|c|}{ hours } \\
\hline Data collection interval & $\begin{array}{l}\text { 100 data points were collected for 1 second at } \\
\text { the beginning of the test. Then, a user setting of } \\
1 \mathrm{~Hz} \text { was used. }\end{array}$ \\
\hline Load & $15 \mathrm{~N}$ \\
\hline Rotational speed & $50 \mathrm{rpm}$ \\
\hline Wear track radius & $5 \mathrm{~mm}$ \\
\hline Test environment & Room temperature \\
\hline
\end{tabular}

\subsubsection{Wear evaluation}

\subsubsection{Wear volume loss}

To depict the cross-sectional profile of the wear track in the worn surface of the alloy specimen, a 2D profilometer, Dektak 150 Surface Profiler, shown in Figure 4-9, was employed. The measuring mechanism is that a needle-shaped probe lightly touches the surface of the worn surface and scans in one direction to depict the topography on the tracking path. For the wear test, therefore, the topography obtained is valley-shaped. An example of the cross-sectional profile of the wear track is illustrated in Figure 4-10, where the valley-shaped profile represents a measured cross-sectional area $\left(\mathrm{mm}^{2}\right)$ of the wear track, so that the volume $\left(\mathrm{mm}^{3}\right)$ of the entire wear track can be estimated by multiplying the perimeter $(\mathrm{mm})$ of the wear track by the measured cross-sectional area $\left(\mathrm{mm}^{2}\right)$. 


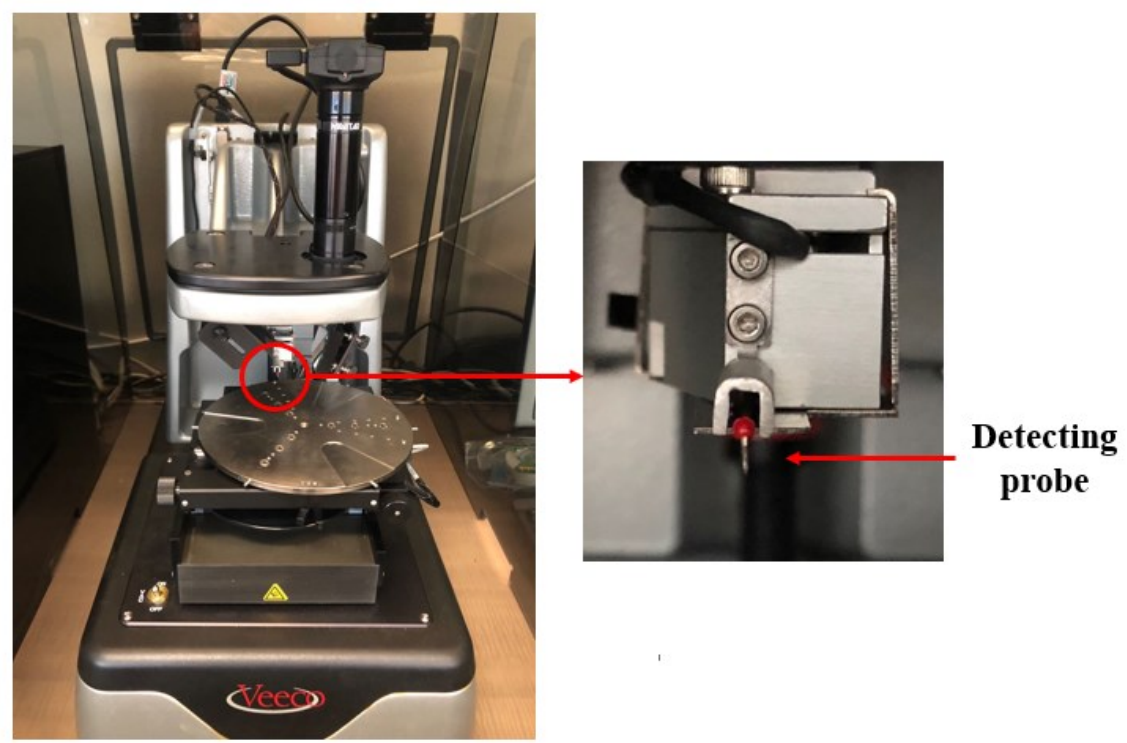

Figure 4-9: Dektak 150 2D surface profiler.

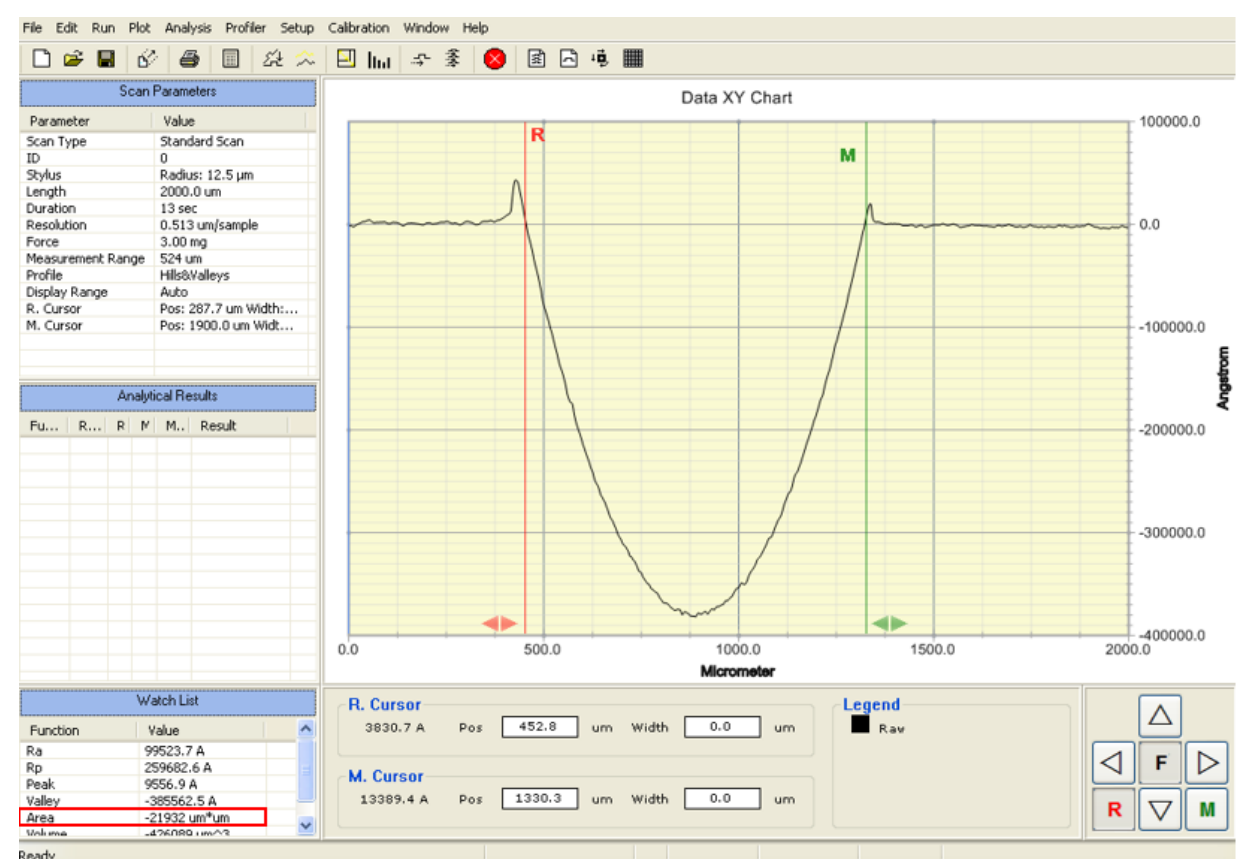

Figure 4-10: An example of cross-sectional profile of wear track from the $2 \mathrm{D}$ profiler.

Wear trails are not perfectly uniform due to the nature of material wear. To estimate the wear volume loss as accurately as possible, the cross-section profile was simulated at four locations 
on each wear track using the 2D profiler, as illustrated in Figure 4-11, and then an average value of wear track volume was obtained as the wear loss of the material.

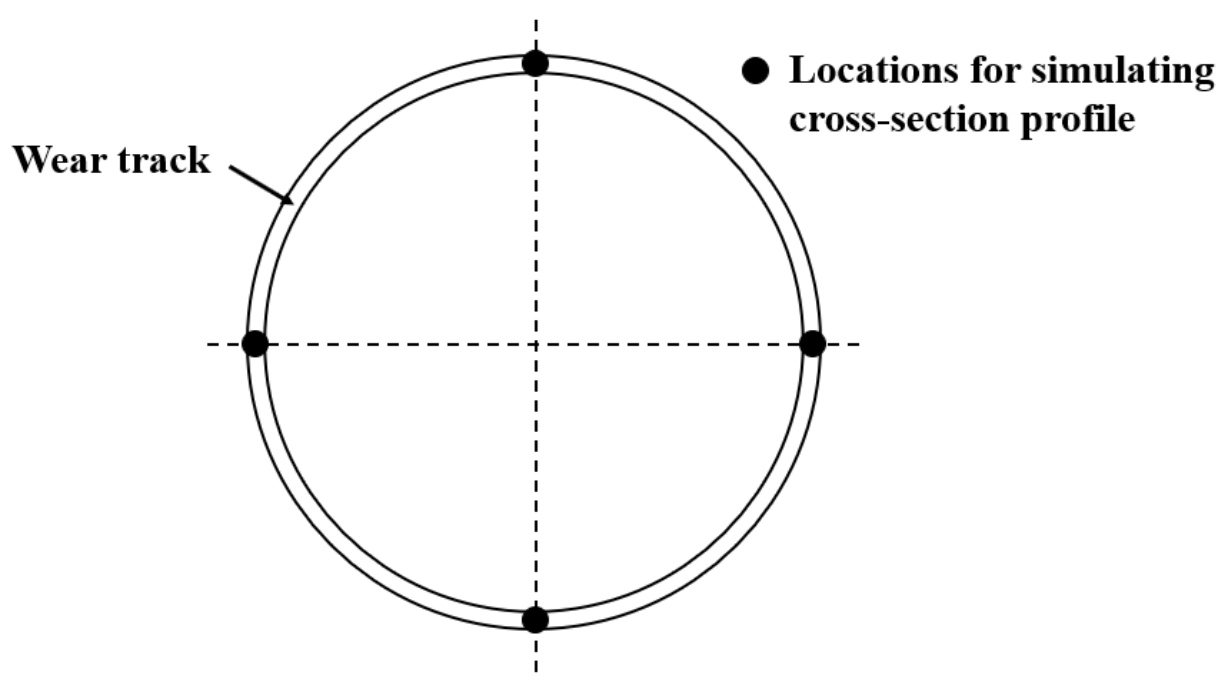

Figure 4-11: Illustration of four measuring locations on a wear track.

Some selected worn specimens are photographically shown in Figure 4-12 to compare visual differences in wear track. For the PTA specimens, PTA Stellite 6 shows a narrower, shallower, and smoother wear band, compared to PTA HE6 specimen, which suggests that the former has less volume loss than the latter. It is also noticed that all the HE6 specimens exhibit a "worm-like" texture of wear track, along with macroscopic black oxidation patches, as seen in Figure 4-12 (b),(d), and (e). For the SPS specimens, similar characteristics of wear tracks are observed. Two SPS HE6 specimens reveal a comparable wear tracks that are larger than that of SPS Stellite 6 specimen. For Stellite 6 alloys, two different fabrication methods show little influence on the wear loss by visual observation. 


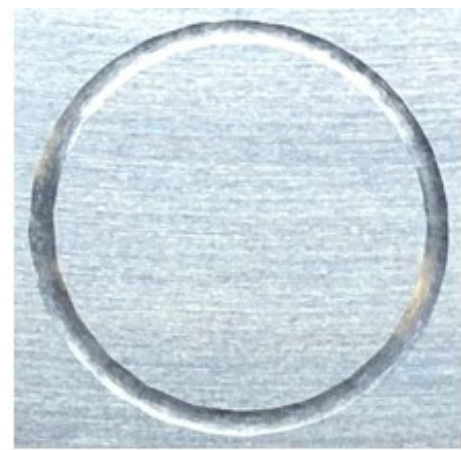

(a)

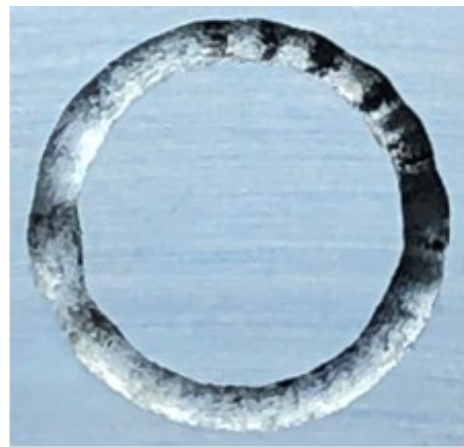

(b)

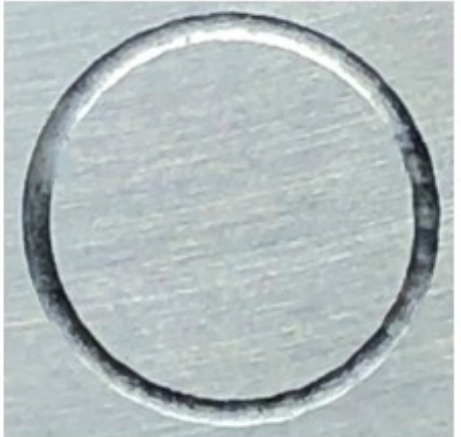

(c)

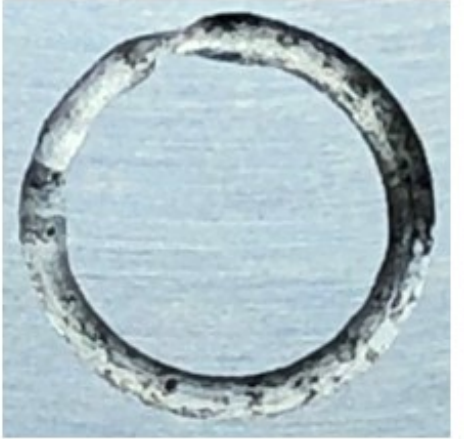

(d)

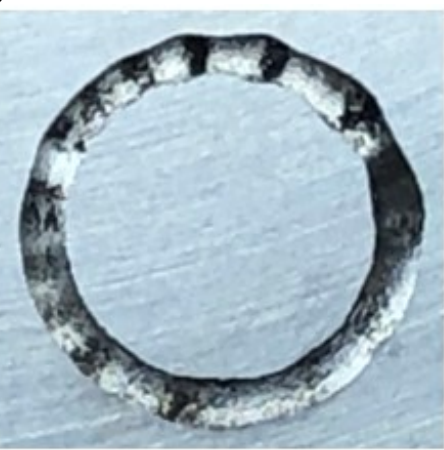

(e)

Figure 4-12: Wear track of (a) PTA Stellite 6, (b) PTA HE6, (c) SPS Stellite 6, (d) SPS HE6 (45 $\mu \mathrm{m}),(\mathrm{e})$ SPS HE6 $(150 \mu \mathrm{m})$.

The cross-sectional profiles of the wear tracks obtained with the 2D profiler are illustrated in Figure 4-13. The profiles reflect a varying degree of width, and associated depth changes on the valley-shaped curves. In general, the wear tracks of the HE6 specimens are much larger than that of the Stellite 6 specimens, which indicate that Stellite 6 has less wear loss than HE6. Comparing the two processing methods, there is no notable difference between the same grade specimens, from the perspective of wear track shape and size. It is clearly shown that Stellite 6 has better wear performance under unlubricated wear condition. 


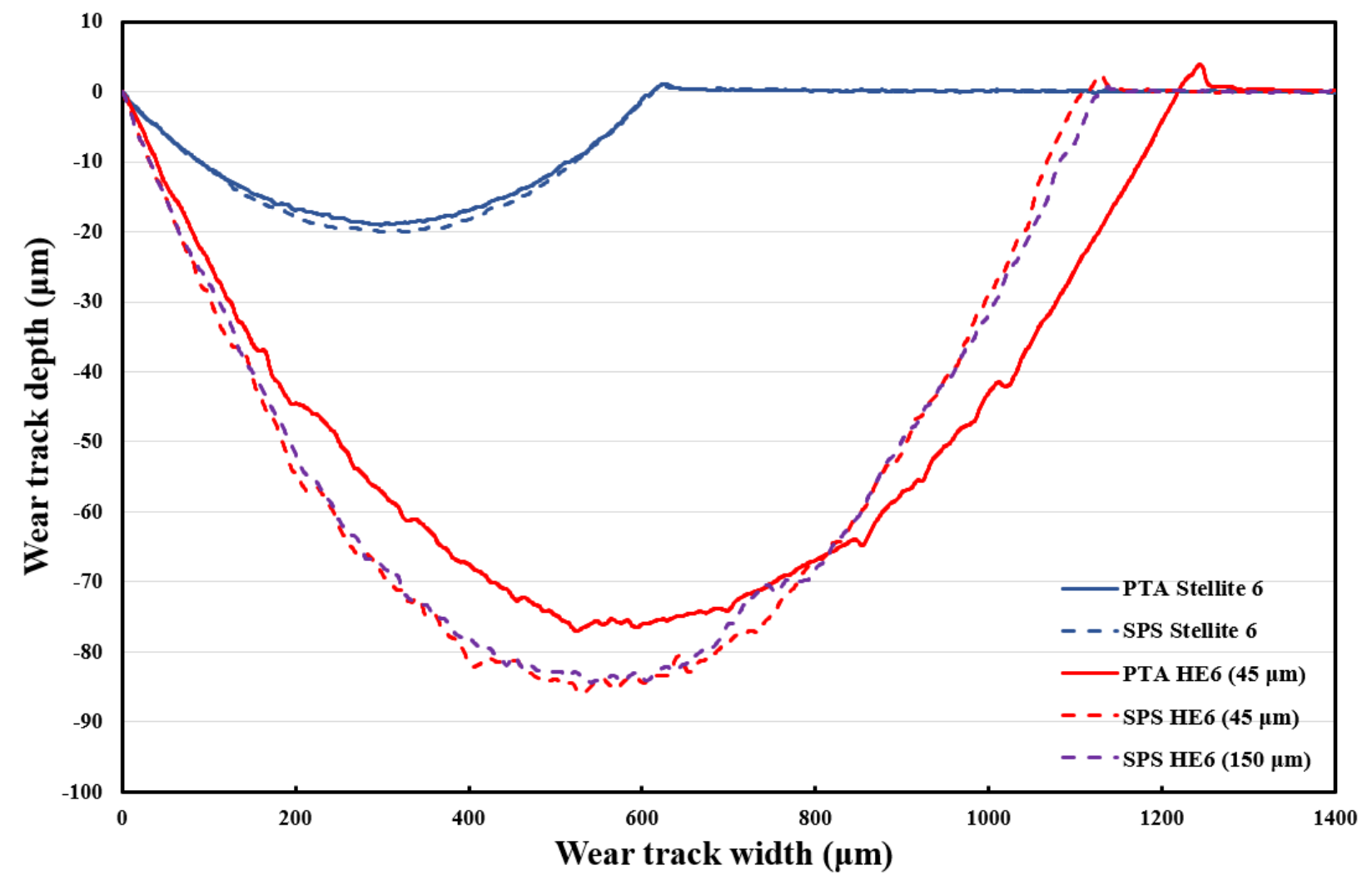

Figure 4-13: Comparison of the cross-sectional profiles of wear tracks between tested alloys.

The average wear volume loss of each alloy specimen was calculated from the wear track profile and the obtained wear loss results are illustrated in Figure 4-14. The volume losses between the two alloy grades demonstrate a significant difference, i.e., HE6 has about an order of magnitude higher loss than Stellite 6 from either manufacturing process. Between the two processing methods, from the average wear loss values, PTA produced the bulk specimens with better wear resistance than SPS, but the testing data scattering (error bars) should also be taken into account. Thus, it is hard to define distinct differences in wear loss between the materials from the two manufacturing processes. Similarly, for the particle size influence on the wear behavior of HE6, although on average the specimen from larger-size powder exhibited better wear resistance, if the testing errors are also considered, it can be suggested that SPS HE6 specimens have similar wear resistance. In 
other words, the particle size of the initial powder has little effect on the SPS specimens with respect to wear resistance.

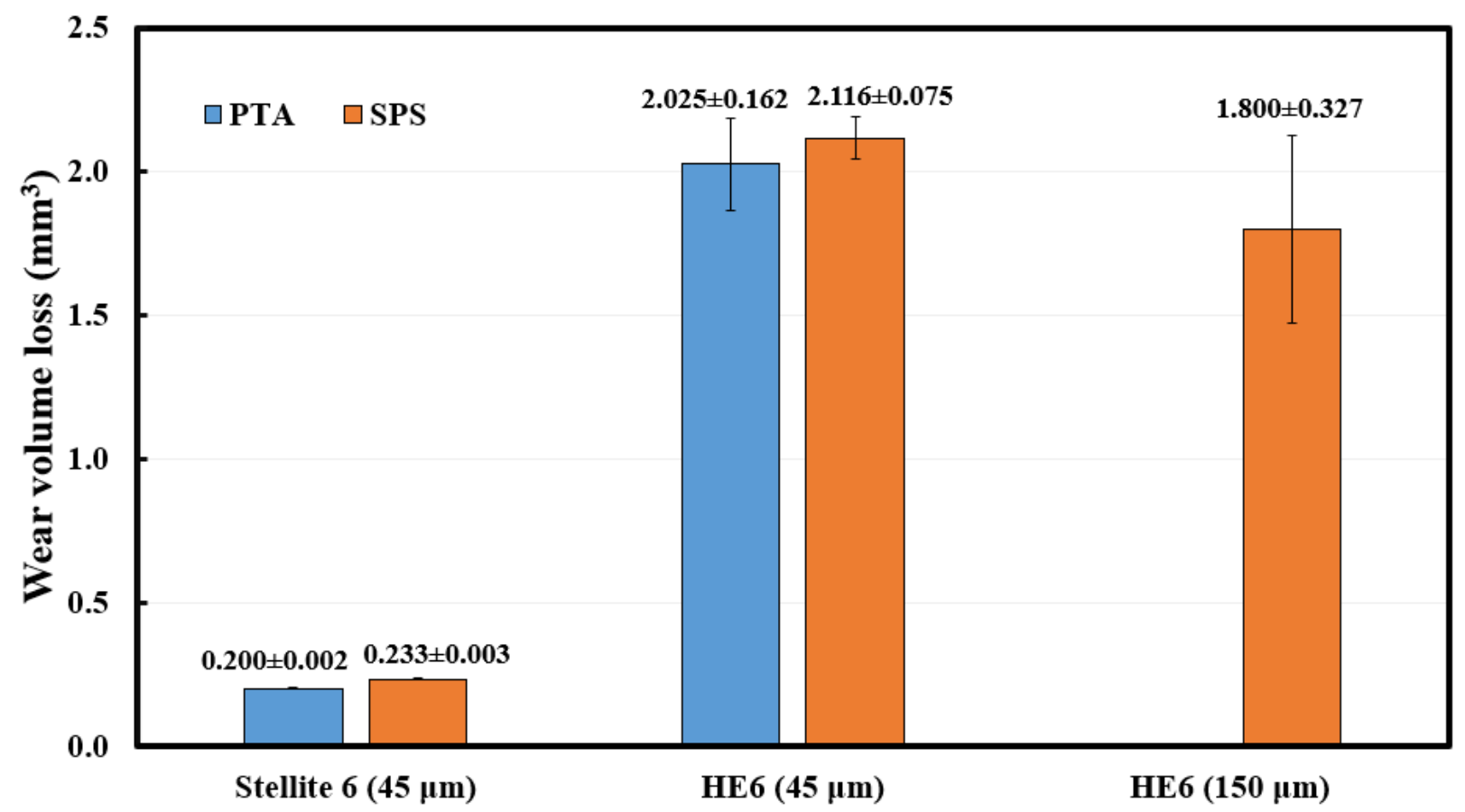

Figure 4-14: Wear volume losses of tested alloys at 50 rpm under $15 \mathrm{~N}$ for 2 hour dry sliding.

\subsubsection{Friction coefficient}

Friction coefficient is one of the parameters that characterize wear behavior of a friction pair. It is defined as a ratio of friction force to the normal force that is applied to the contacting surface (Equation 4.2). It reflects the roughness of the contacting surfaces, with rougher surfaces tending to have a larger friction value. In the present study, friction forces were automatically recorded by the pin-on-disc wear tester software during sliding wear tests, and the corresponding friction coefficients were then calculated.

The friction coefficients in real-time for the alloy specimens are illustrated in Figure 4-15. Overall, the Stellite 6 specimens have a smaller friction coefficient, compared with the HE6 
specimens, which corresponds to the wear loss results showing superior wear performance of Stellite 6 to HE6. For the same grade alloy processed via PTA and SPS, respectively, there is no obvious difference in the friction coefficient curves at a stable state. Moreover, the difference in powder size does not affect the trend of friction coefficient for HE6 alloy by comparing the SPS HE6 $(45 \mu \mathrm{m})$ and SPS HE6 $(150 \mu \mathrm{m})$ specimens.

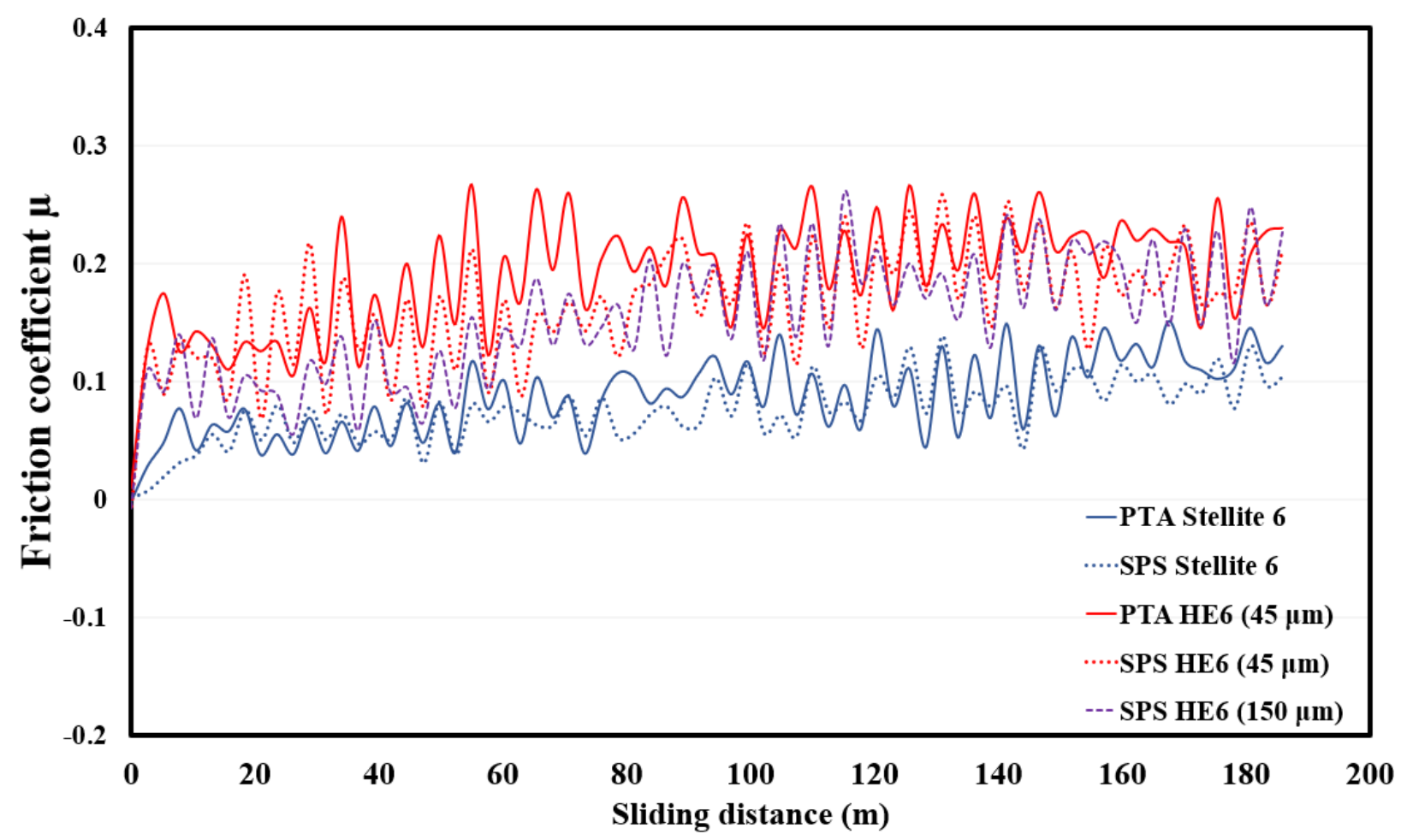

Figure 4-15: Friction coefficient versus sliding distance.

The average values of friction coefficient for the tested specimens are presented in Figure 4-16. Evidently, HE6 specimens have much higher values of friction coefficient than Stellite 6 from either processing method. However, for the same grade alloy processed via different approaches, the SPS specimens show slightly lower friction than the PTA ones. Meanwhile, for SPS HE6 specimens, the one from larger-size powder has a slightly larger value of friction 
coefficient than that from smaller-size powder. Considering the individual difference of each specimen, the difference in friction coefficient between the two SPS HE6 specimens is negligible.

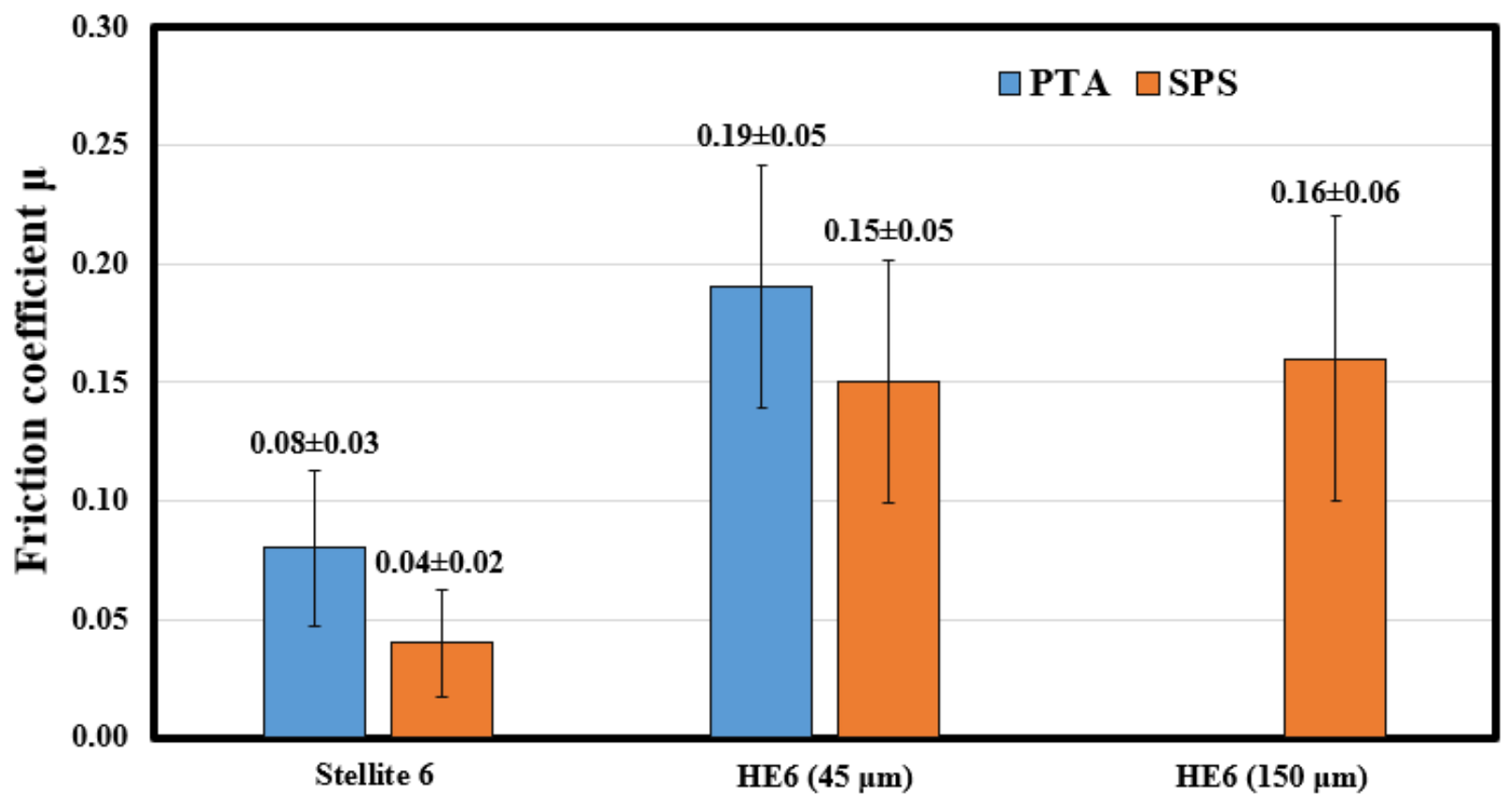

Figure 4-16: Average friction coefficients of tested alloys.

\subsubsection{Worn surface}

To better understand the wear behavior of Stellite 6 and HE6, SEM and EDX were employed to examine the worn surfaces of the alloy specimens. The obtained images of worn surface morphology are presented in Figure 4-17 to Figure 4-23. Both secondary electron (SE) and backscattered electron (BSE) imaging modes were used in the SEM/EDX analyses. Due to the different mechanism of imaging principles, SE can better form an image reflecting the threedimensional topography, while BSE is more preferred in elemental analysis. Moreover, from previous research [7], bright area in the SE mode usually associates with oxidized parts because electrons concentrate on the parts which are not conductive and reveal the brightness in SE imaging. 
Figure 4-17 to Figure 4-20 present the worn surface morphologies of Stellite 6 specimens processed via PTA and SPS, which are characterized by both abrasive and adhesive wear mechanisms. Scratches or scars (ploughing) are found on the wear track of both specimens. Also, material deformation and oxidation had occurred in the surfaces during the wear tests. The red circles (Figure 4-17 c) mark the oxidized areas caused by friction heat during the wear process. According to the surface morphologies in Figure 4-18 b and c, SPS Stellite 6 has smaller wear chips than PTA Stellite 6 which shows larger bulk material chipped off. The morphology of the wear track in PTA Stellite 6 surface is featured by constant oxidation and deformation. Compared with PTA Stellite 6 specimen surface, SPS Stellite 6 specimen surface shows slightly shallower wear scars (Figure 4-18 c) although oxidation and deformation are also the main wear mechanism of this alloy.

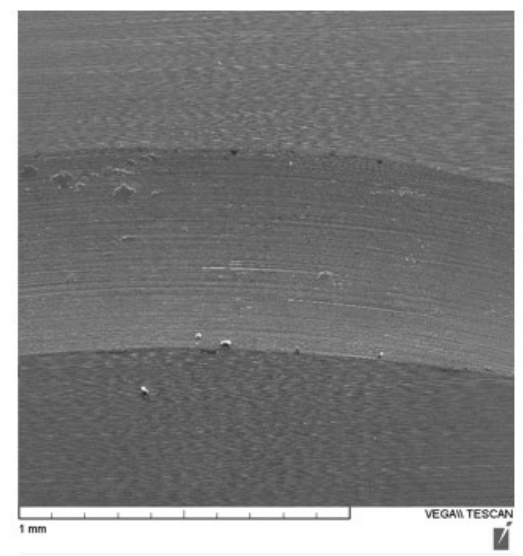

(a)

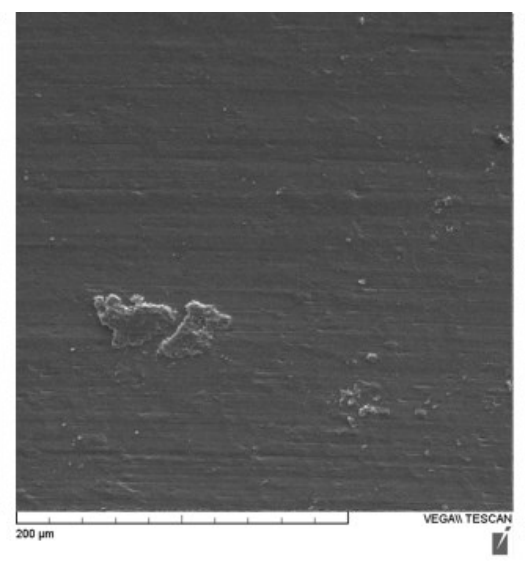

(b)

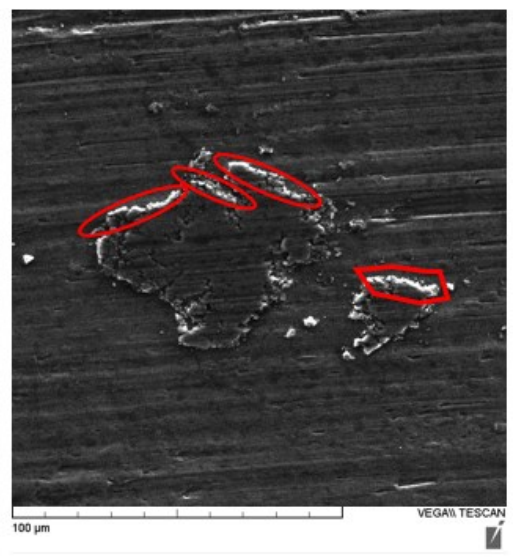

(c)

Figure 4-17: SE image of worn surface of PTA Stellite 6 at (a) low magnification, (b) medium magnification, (c) high magnification. 


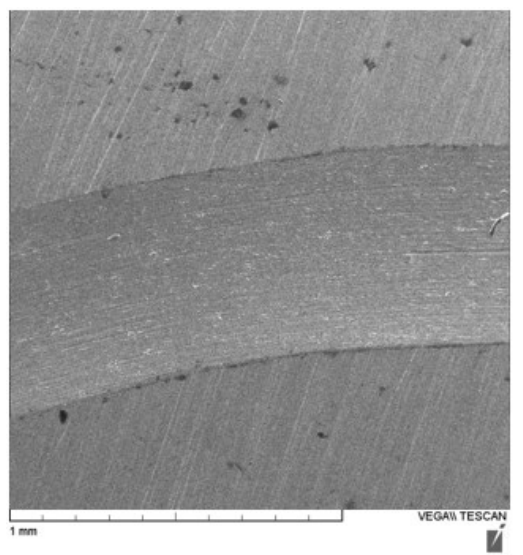

(a)

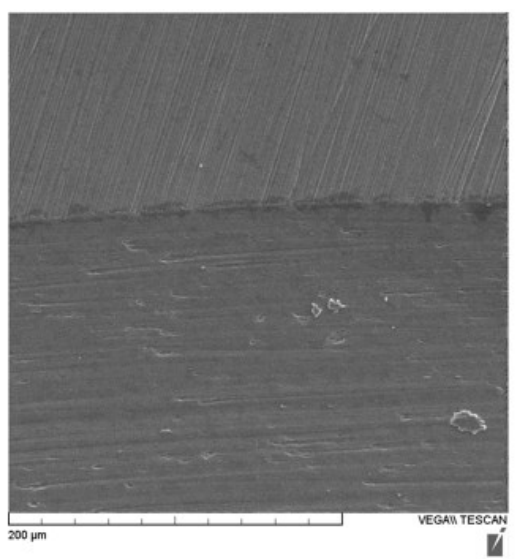

(b)

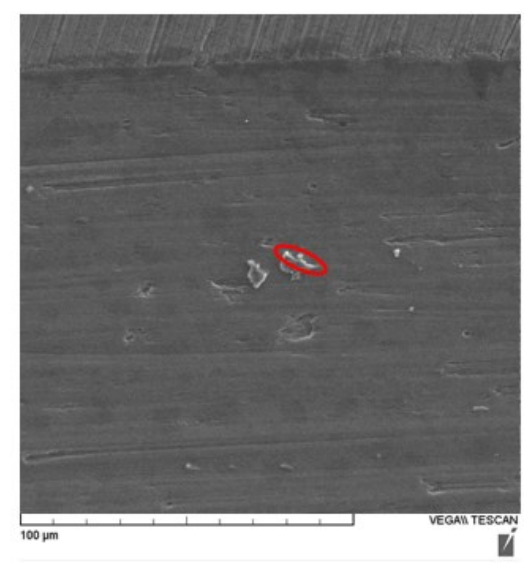

(c)

Figure 4-18: SE image of worn surface of SPS Stellite 6 at (a) low magnification, (b) medium magnification, (c) high magnification.

Figure 4-19 and Figure 4-20 further display the worn surface morphologies of the Stellite 6 specimens with BSE images to reveal the microstructure-related wear mechanism. In Figure 4-19, it seems that the carbides were oxidized much more than the solid solution phase on PTA Stellite 6 surface during the wear test. Hence, the distinct segregation of different phases in terms of oxidation further developed pits and debris in the worn surface. Since SPS Stellite 6 surface has much finer carbide/intermetallic phases that are more homogeneously distributed than the carbide phase in PTA Stellite 6 surface, SPS Stellite 6 has smoother worn surface that may prevent disastrous surface degradation in longer wear. 


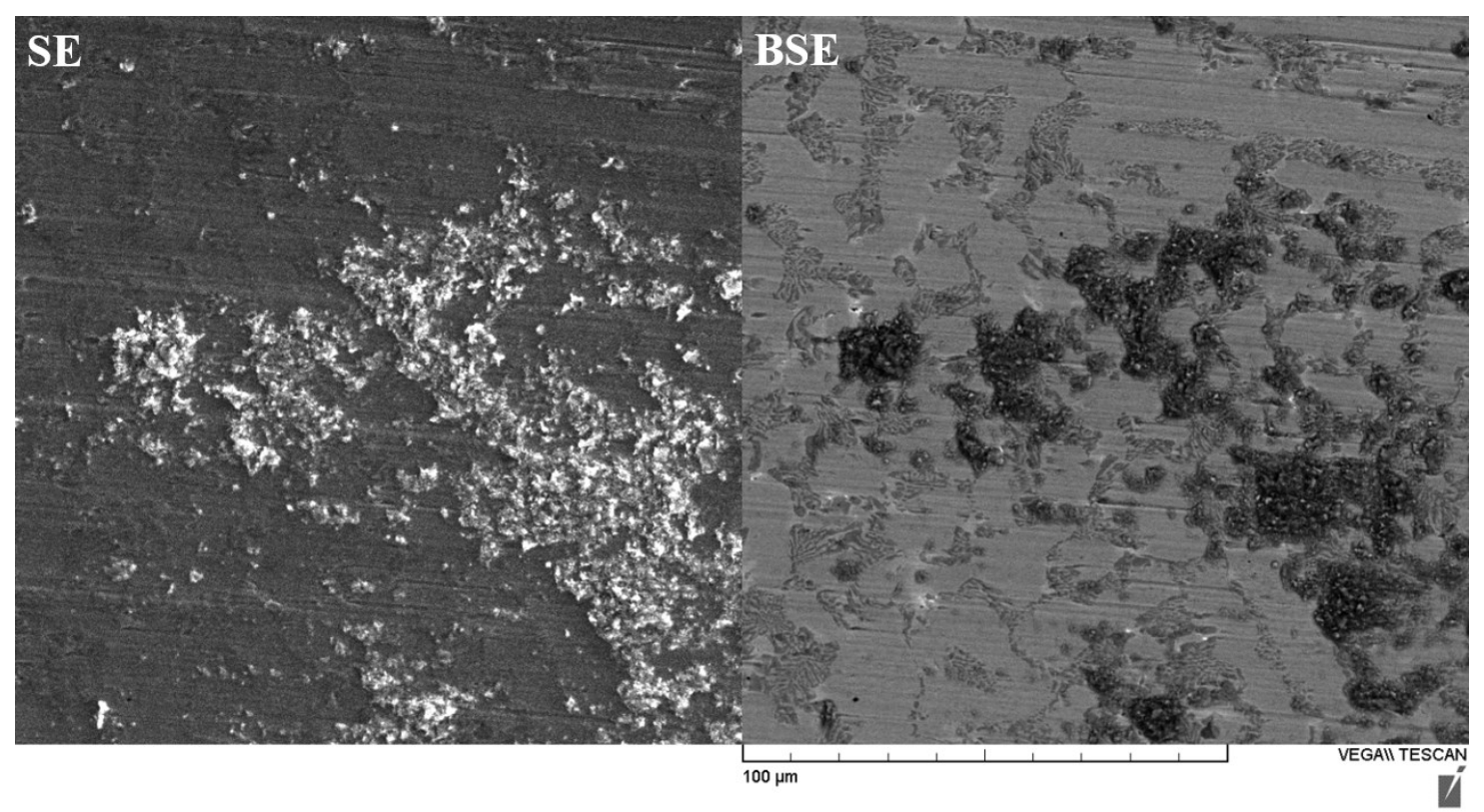

Figure 4-19: SE and BSE images of wear track of PTA Stellite 6.

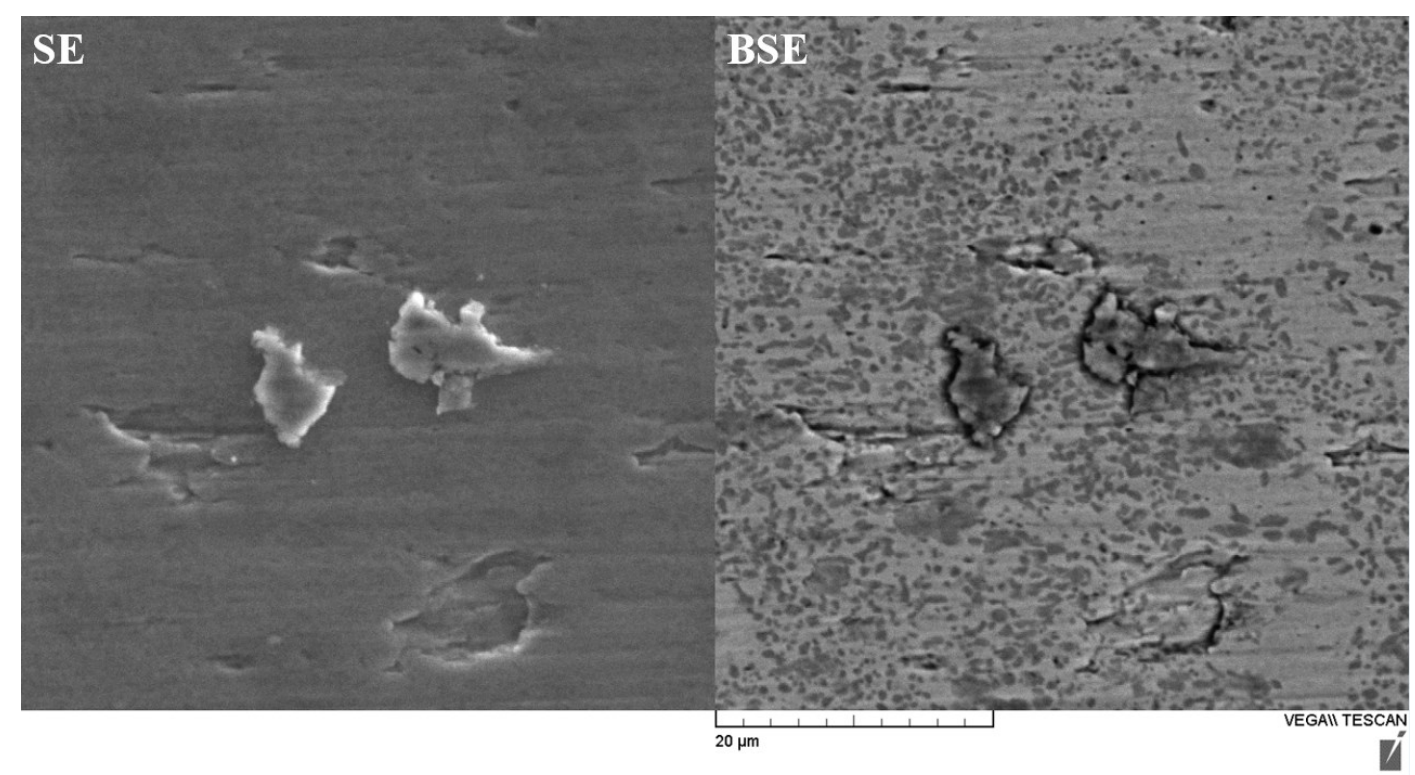

Figure 4-20: SE and BSE images of wear track of SPS Stellite 6.

According to the worn surface morphologies in Figure 4-21 to Figure 4-23, severe plastic deformations had occurred in the surface of HE6 specimens during the wear tests, accompanied by a certain degree of oxidation, as highlighted with red circles in the images. The SE/BSE images 
of HE6 worn surfaces are presented in Figure 4-24 to Figure 4-26. For PTA HE6 surface, the carbides/intermetallics were drastically damaged under the wear test. Not only the eutectics but also the solid solution were deformed and chipped off. Similar behavior was found in the worn surfaces of SPS HE6 $(45 \mu \mathrm{m})$ and SPS HE6 $(150 \mu \mathrm{m})$ specimens even though they have carbides/intermetallics in different ratios and morphologies in comparison with PTA HE6.

Compared with Stellite 6, HE6 surfaces obviously experienced more severe damage. Worm-like contours around the periphery of the wear track were generated in the HE6 surfaces during the wear tests, which were characterized by apparent narrowing and widening regions having varying depth. This behavior was likely attributed to some of the oxidized debris stuck in the wear tracks, which was pushed by the wearing ball. The hard build-ups of oxides in the wear track were regarded as pads making the $\mathrm{Si}_{3} \mathrm{~N}_{4}$ ball move up and down. Then, a certain morphology of the wear track was formed.

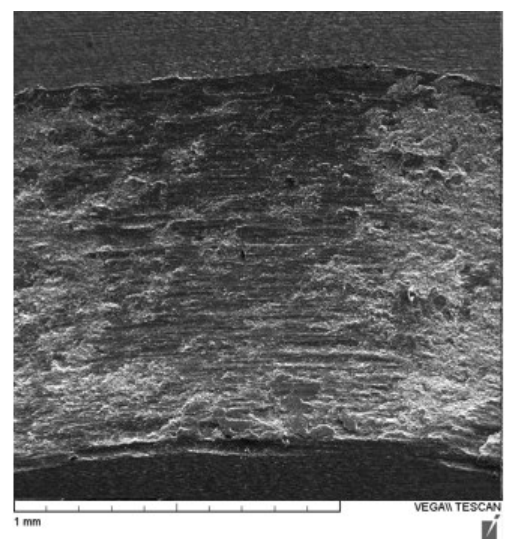

(a)

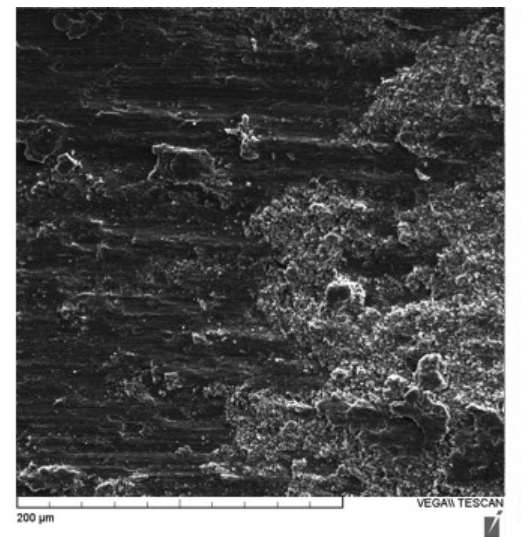

(b)

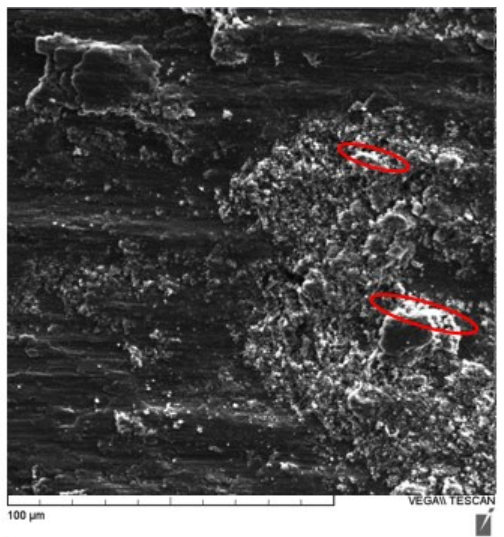

(c)

Figure 4-21: SE image of worn surface of PTA HE6 at (a) low magnification, (b) medium magnification, (c) high magnification. 


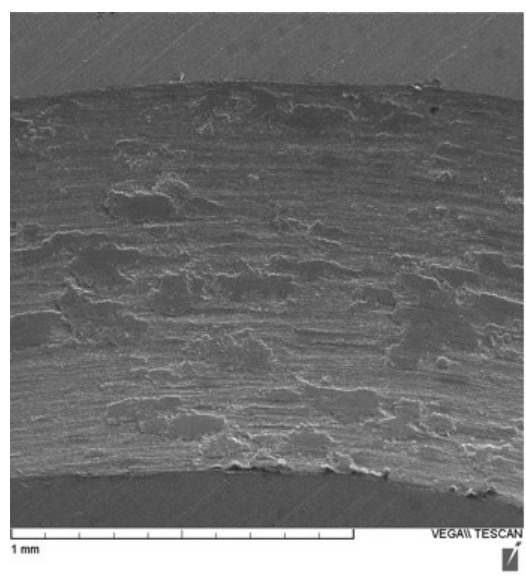

(a)

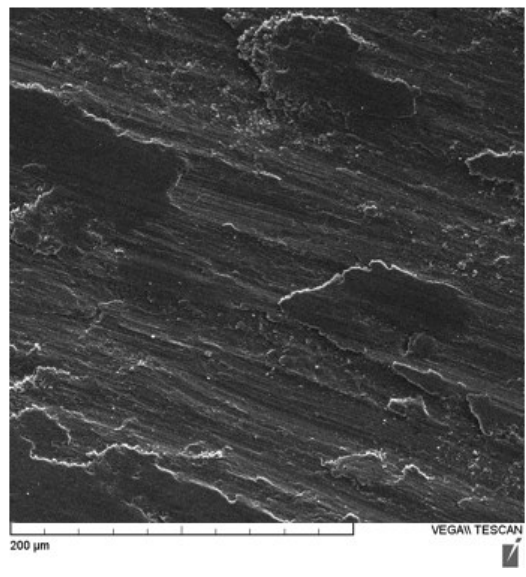

(b)

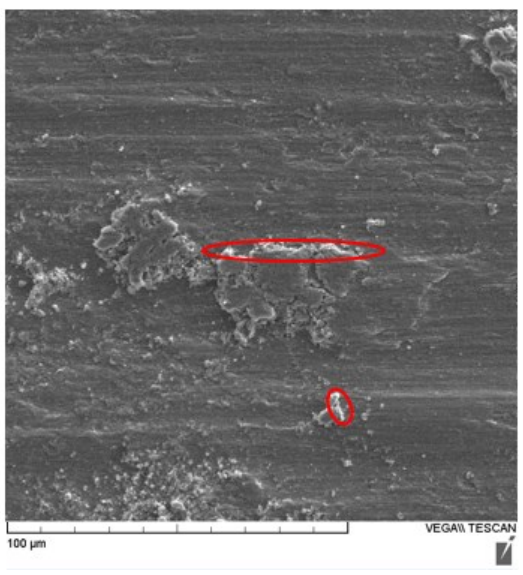

(c)

Figure 4-22: SE image of worn surface of SPS HE6 (45 $\mu \mathrm{m})$ at (a) low magnification, (b) medium magnification, (c) high magnification.

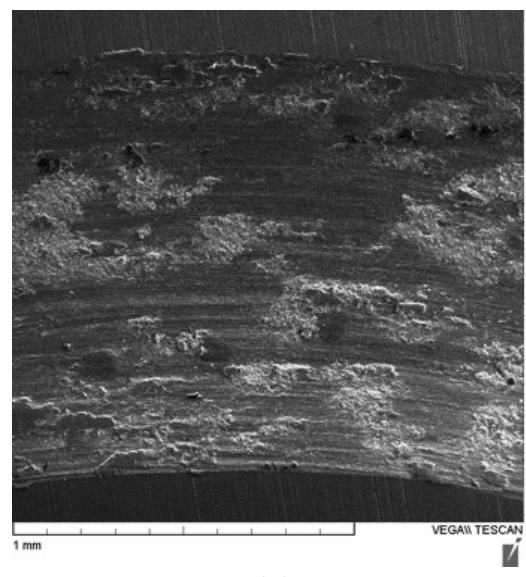

(a)

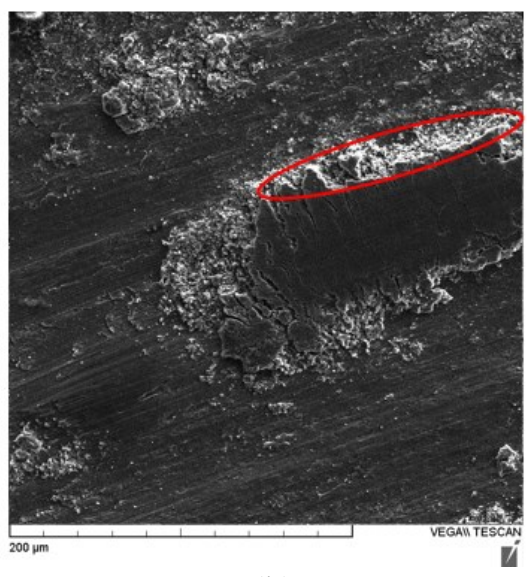

(b)

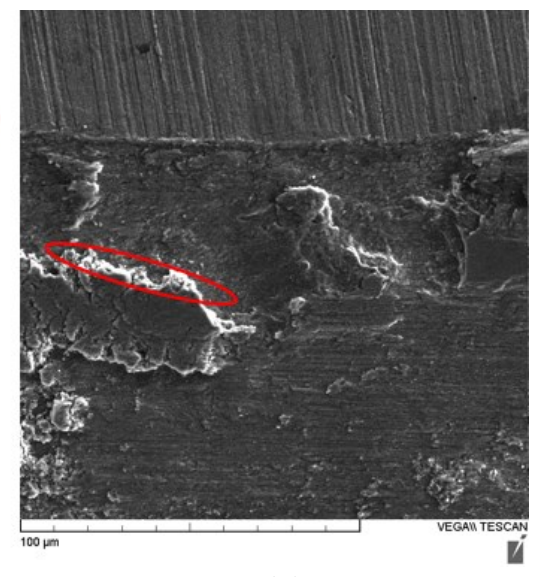

(c)

Figure 4-23: SE image of worn surface of SPS HE6 $(150 \mu \mathrm{m})$ at (a) low magnification, (b) medium magnification, (c) high magnification. 


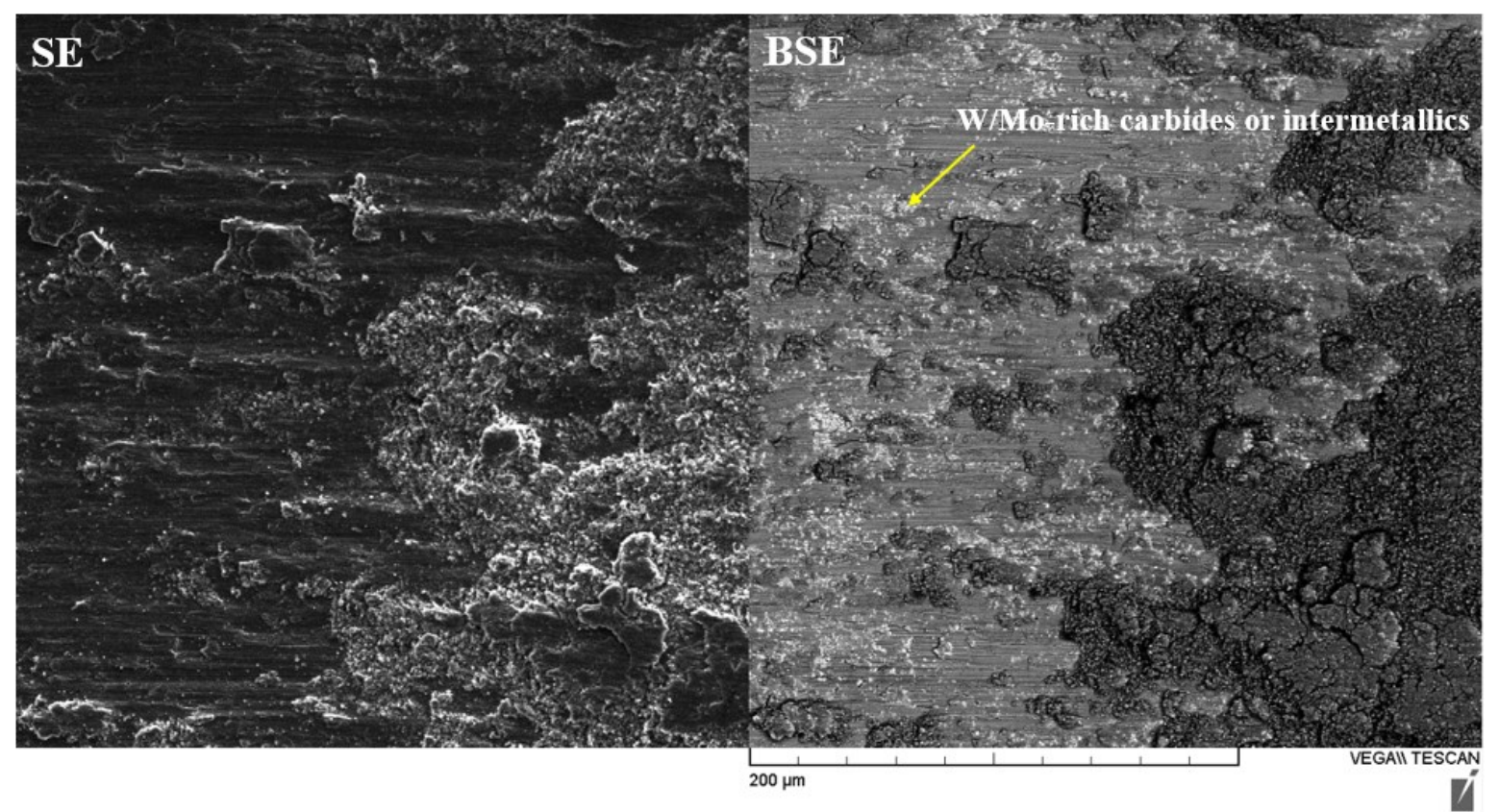

Figure 4-24: SE and BSE images of wear track of PTA HE6.

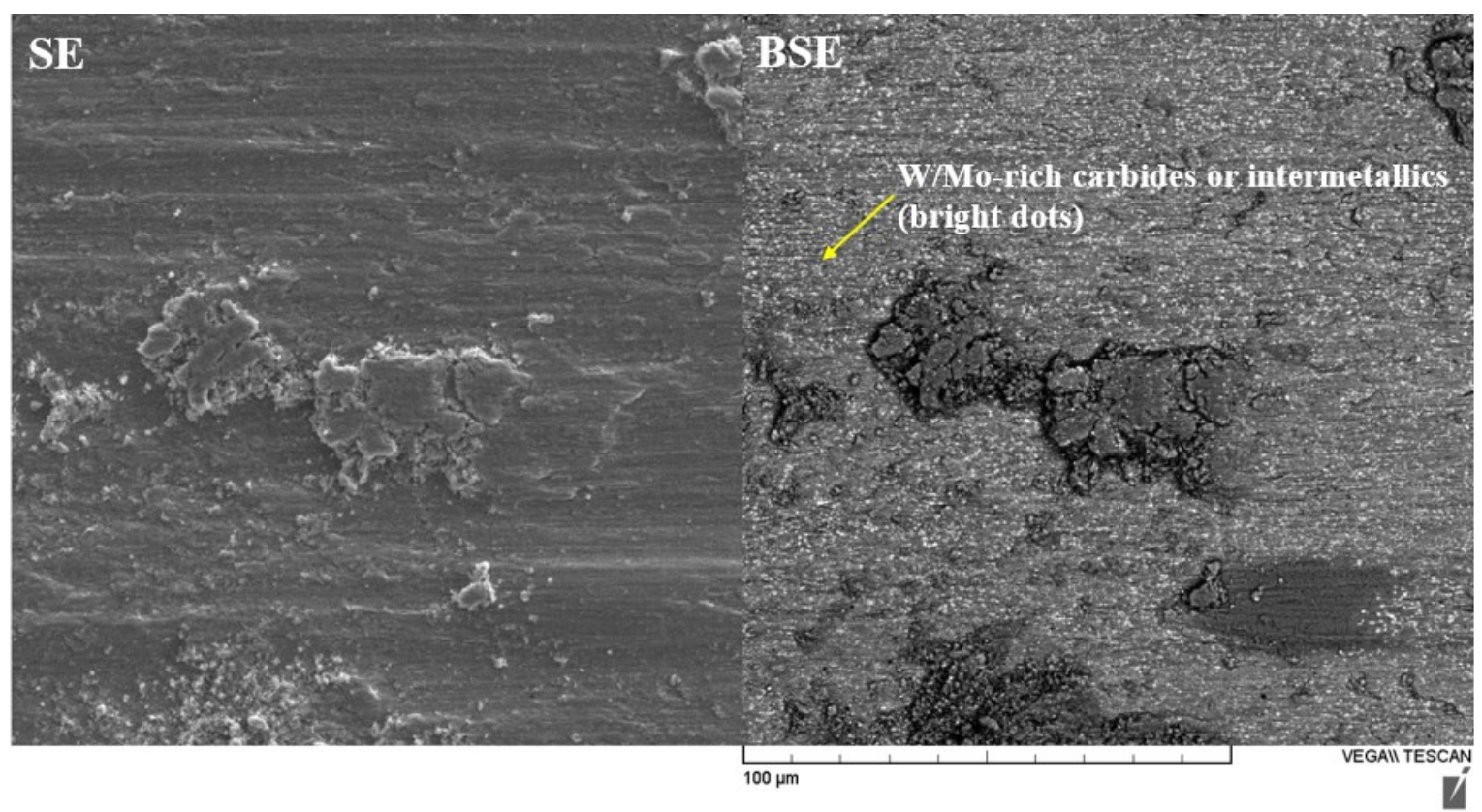

Figure 4-25: SE and BSE images of wear track of SPS HE6 $(45 \mu \mathrm{m})$. 


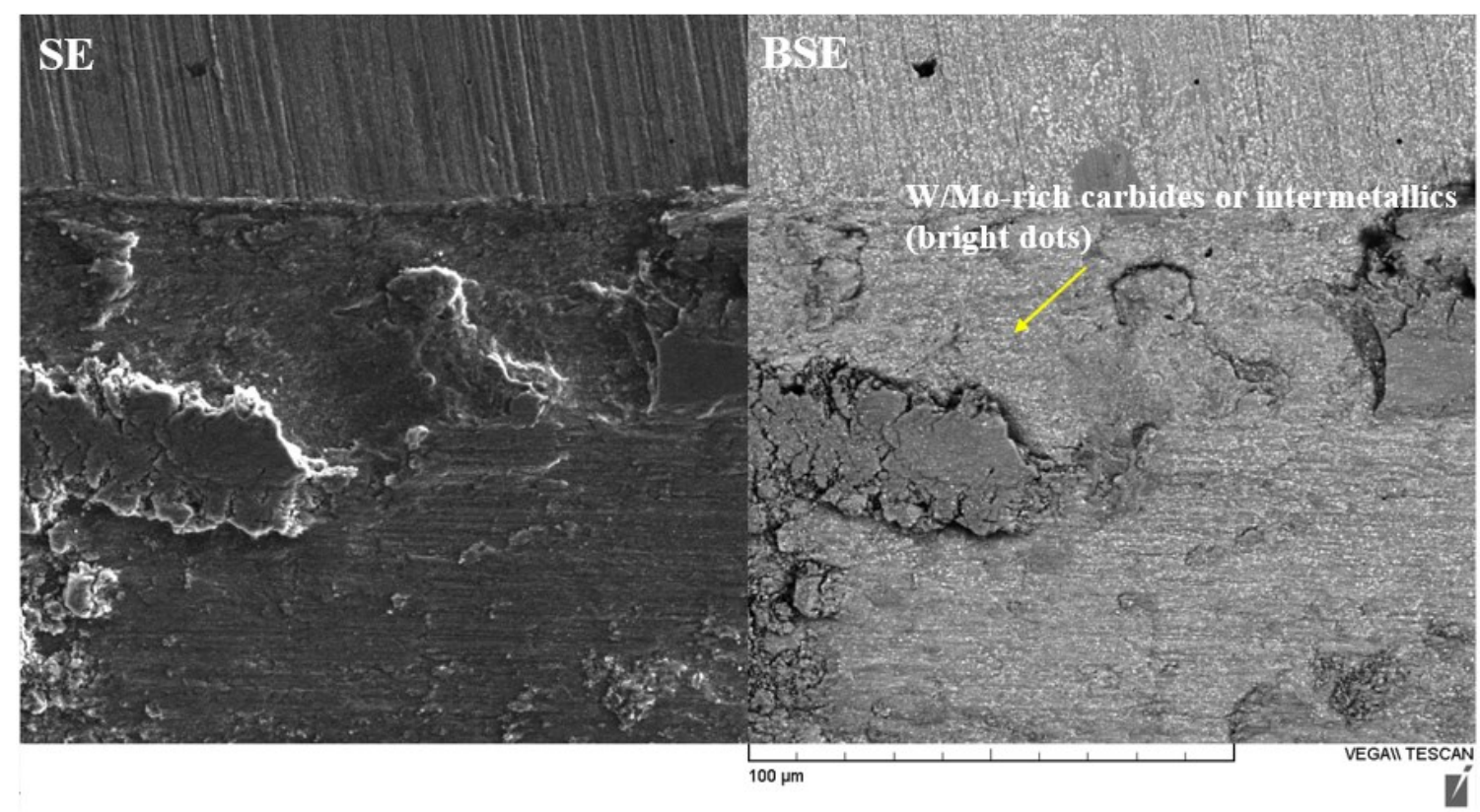

Figure 4-26: SE and BSE images of wear track of SPS HE6 $(150 \mu \mathrm{m})$.

\subsubsection{Wear coefficient}

In the present study, another well-recognized parameter that estimates the degree of material wear in the industry is wear coefficient. When a wear process starts, there is a breakingin stage for a worn pair which is regarded as an unsteady state. It is difficult to measure or predict the wear performance in this stage. However, once the wear system reaches a steady state, the wear coefficient can be utilized to analyze the wear performance of materials in a wear test. In this research, the wear coefficient per unit sliding distance was calculated by the Archard Equation [93]:

$$
K=\frac{w H}{P}
$$

where $K$ refers to the wear coefficient, $w$ is the wear volume loss per distance $\left(\mathrm{mm}^{3} / \mathrm{m}\right), H$ is the hardness of the material (HRC) and $P$ is the applied load (N). 
In terms of wear coefficient, an approximate scale can be used to compare numerous materials in wear resistance. From the results of wear coefficient for tested alloys, Stellite 6 alloy tends to have lower wear coefficient compared to HE6 in the wear test, no matter which processing method was used to create the bulk specimens. This is in full agreement with the wear loss results and proves the consistency of the wear coefficient and wear volume loss. Comparing the effect of the processing method on the wear of the same material, the wear coefficient of SPS specimen is slightly higher than that of PTA one, which implies that the SPS specimen tends to have more wear loss. The wear coefficient results are consistent with the wear loss results. The influence of the initial powder size on the wear performance of SPS HE6 specimens is also reflected in Figure 4-27. SPS HE6 specimen from larger-size powder has a smaller wear coefficient, but considering its larger standard deviation range, the two SPS specimens are still at the same level of wear coefficient. However, in general, the alloying powder with smaller particle size produced better quality assurance.

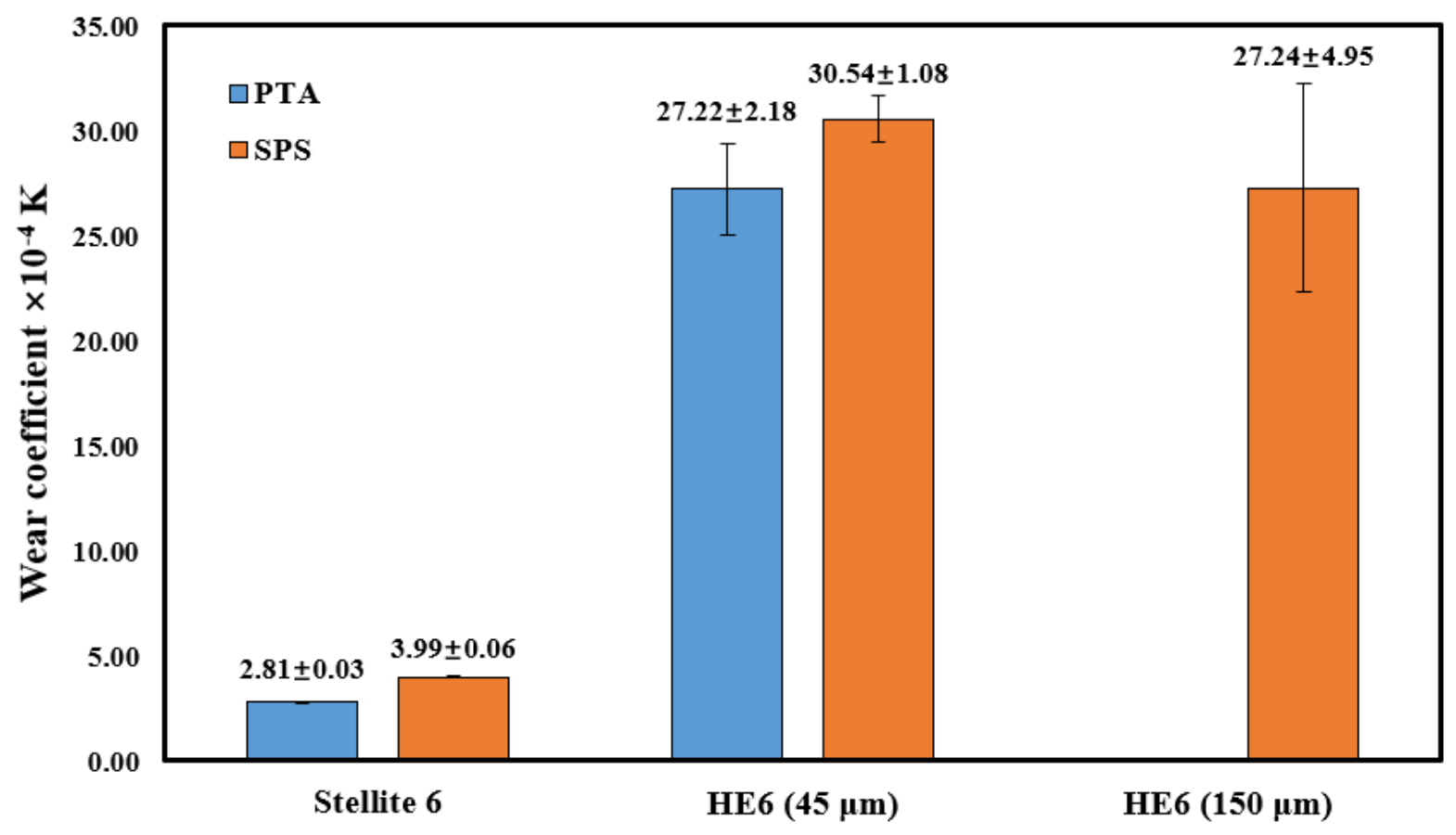

Figure 4-27: Wear coefficients of tested alloys. 


\subsection{Corrosion Test}

The corrosion behavior of PTA specimens of Stellite 6 and HE6 was investigated in different media, such as $3.5 \% \mathrm{NaCl}, 2 \% \mathrm{HCl}$ and $10 \% \mathrm{H}_{2} \mathrm{SO}_{4}$ (in weight), using electrochemical test and immersion test. Electrochemical testing is an analytical chemistry method through measuring the potential and current of an electrochemical system. By applying a voltage on a material specimen, the corrosion rate can be accelerated, hence, the corrosion behavior of the material can be characterized and analyzed in a fast manner. Electrochemical Impedance Spectroscopy (EIS) and polarization belong to this category. EIS is a technique utilized in a static state, i.e., a very small fixed AC amplitude (often $10 \mathrm{mV}$ ) is applied. It provides information about the surface oxide layer of a specimen by measuring the response to the applied alternating current (AC) power on the specimen surface. In contrast, polarization measures the current density change within a changing voltage scanning range that is powered by direct current (DC). Since the DC power of polarization is often high enough to destroy the specimen surface, polarization is considered as a destructive method, while EIS is categorized as a non-destructive technique. Several parameters measured during polarization test are used to characterize general and localized corrosion behavior of the material, such as corrosion potential $\left(E_{\text {corr }}\right)$, current density $\left(I_{\text {corr }}\right)$ and polarization resistance $\left(R_{p}\right)$, etc.

Immersion test is a conventional method that simply soaks the material specimen in the desired chemical solution for a period of time, which can simulate the real working environment corrosion to some extent. The parameters, such as the concentration of the chemical solution, temperature, immersion duration, and so on, often affect the test results and should be carefully selected. Moreover, SEM is also employed to assist the understanding of the corrosion mechanisms of the tested material. 


\subsubsection{Electrochemical corrosion tests}

Cyclic potentiodynamic polarization and EIS methods were used on PTA Stellte 6 and PTA HE6 specimens in three different chemical solutions, which were $3.5 \% \mathrm{NaCl}, 2 \% \mathrm{HCl}$, and $10 \%$ $\mathrm{H}_{2} \mathrm{SO}_{4}$ (in weight).

\subsubsection{Specimen and solution preparation}

The surfaces of PTA Stellite 6 and PTA HE6 specimens were polished through a series of sandpapers and suspensions to reach the requirements of electrochemical tests, according to ASTM Standard G59 - 97 [94]. Then, the surfaces were cleaned with an ultrasonic cleaner for 15 minutes.

Aqueous saline $(3.5 \% \mathrm{NaCl})$, hydrochloric acid $(2 \% \mathrm{HCl})$, sulfuric acid $\left(10 \% \mathrm{H}_{2} \mathrm{SO}_{4}\right)$ were prepared as the electrochemical agents. $500 \mathrm{ml}$ solution was diluted from high concentration chemical agents, i.e., 99.9\% sodium chloride crystal powder, 37\% hydrochloric acid, and 98\% sulfuric acid, respectively; $17.5 \mathrm{~g} \mathrm{NaCl}$ crystal powder, $57 \mathrm{ml} \mathrm{37 \%} \mathrm{HCl}$, and $30 \mathrm{ml} 98 \% \mathrm{H}_{2} \mathrm{SO}_{4}$ were diluted with distilled water to achieve the specific concentration for each solution.

\subsubsection{Testing systems}

For the electrochemical tests, ASTM Standard G3-14 [95] and ASTM Standard G5-14 [96] were complied. A Solartron 1287A potentiostat (including waveform generator) and 1255B Frequency Response Analyzer, together with K0235 flat cell, were employed. The potentiostat provided direct voltage and worked for the cyclic potentiodynamic tests. The frequency response analyzer can provide alternative voltage and then measure the response with respect to the sinusoidal excitation signal [75], which can be coupled with the Potentiostat for the EIS test. The

electrochemical testing system is shown in Figure 4-28. CorrWare ${ }^{\circledR}$ and CView ${ }^{\mathrm{TM}}$ software 
packages were bundled to execute cyclic potentiodynamic tests. EIS was conducted using Zplot and Zview software packages.

A three-electrode mode was used in this study: a working electrode (WE) that was directly attached to the testing specimen, a counter electrode (CE) which was made of graphite, and a reference electrode (RE) which was saturated calomel electrode (SCE) type, as shown in Figure 4-28 (b). A schematic diagram of the corrosion cell is illustrated in Figure 4-29 [97] to better demonstrate the test setup.

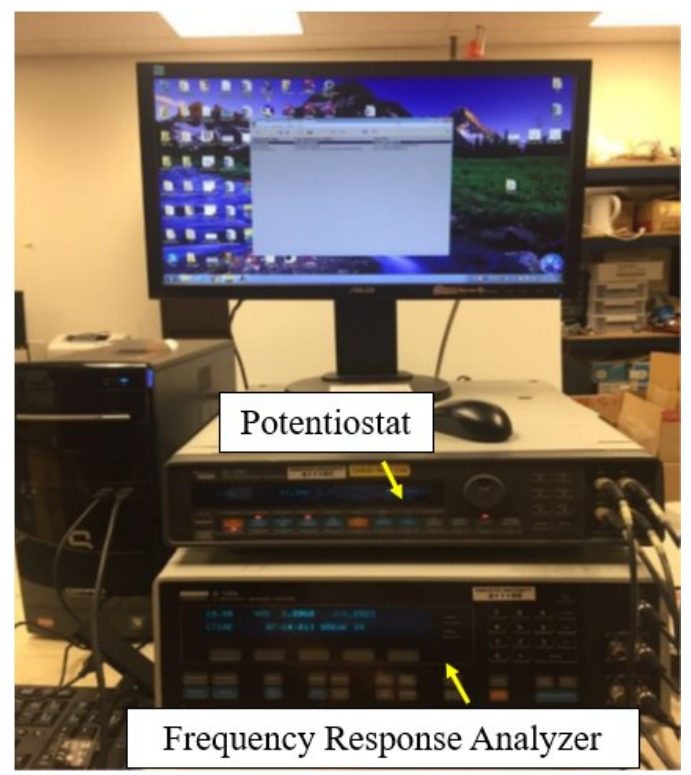

(a)

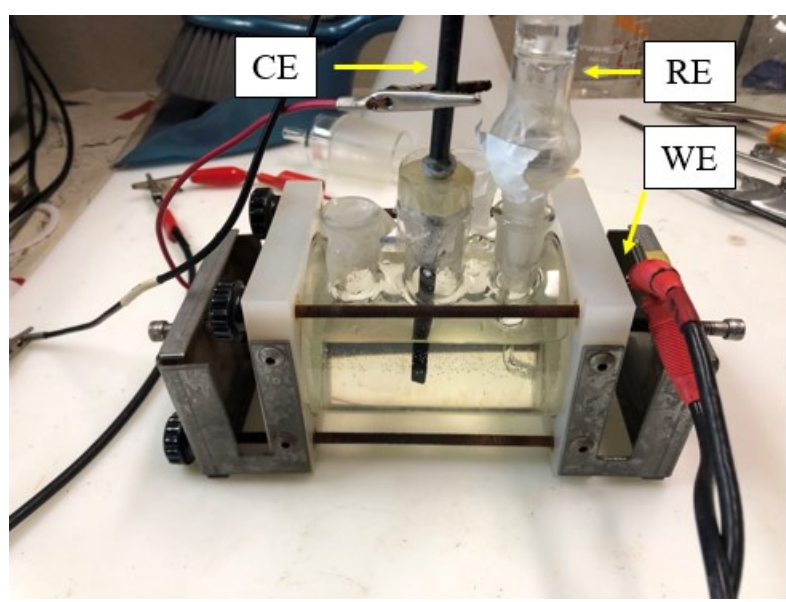

(b)

Figure 4-28: Electrochemical corrosion system setup: (a) control system, (b) K0235 flat corrosion cell. 


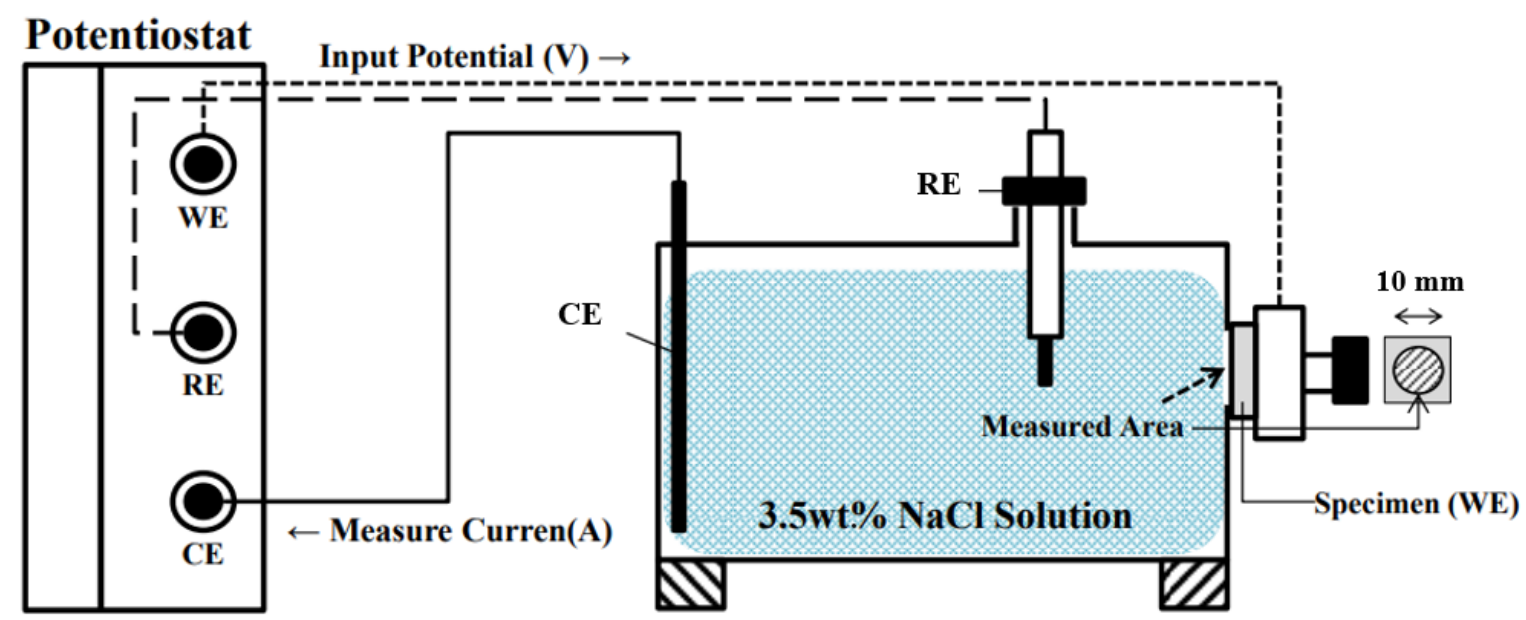

Figure 4-29: Schematic diagram of the electrochemical cell in a $3.5 \mathrm{wt} . \% \mathrm{NaCl}$ corrosion test [97].

Before applying any power voltages on the specimen, open circuit potential (OCP) was firstly monitored for one hour to achieve a quasi-static state. Then EIS was conducted on the specimen, with the frequency starting from $1 \mathrm{E} 6 \mathrm{~Hz}$ to $0.01 \mathrm{~Hz}$ [88]. Finally, the cyclic polarization scan was performed from $-0.5 \mathrm{~V}$ to $1.2 \mathrm{~V}$ and back to $-0.5 \mathrm{~V}$ at a scan rate of $0.1667 \mathrm{mV} / \mathrm{s}$. These scan ranges were determined according to ASTM Standard G5 [96], G59 [94], G102 [74] and previous studies in corrosion test [88][98].

\subsubsection{Open circuit potential}

Determination of OCP is regarded as the standard procedure of the electrochemical system for two purposes. One is to stabilize the electrochemical system that allows the specimen to reach a quasi-equilibrium in a specific chemical solution. The other is that the quasi-static OCP value can be used to determine the starting potential of the polarization test. Figure 4-30 shows the OCP for Stellite 6 and HE6 specimens in different chemical solutions. According to ASTM Standard $\mathrm{G} 3$ and G5, the lower limit of the scan range has to be $-0.3 \mathrm{~V}$ lower than the OCP, and the higher 
limit should be $1 \mathrm{~V}$ higher than it. From the one-hour OCP results, the values of quasi-static potential for all specimens were in a range of -0.1 to $-0.35 \mathrm{~V}$, a very small fluctuation. Thus, for all polarization tests, the starting point of potential was set to $-0.5 \mathrm{~V}$ and the higher limit was 1.2 $\mathrm{V}$.

In addition, based on previous research [98], quasi-static OCP values may provide a sort of intuitive sign for the potentiodynamic corrosion performance of the specimen. From the perspective of thermodynamics, a more positive value of OCP reflects a smaller difference between the equilibrium potential of the cathode and anode reactions, as well as a lower corrosion rate. By comparing the OCP values, PTA Stellite 6 tends to have a slightly better performance in $3.5 \% \mathrm{NaCl}$ and $2 \% \mathrm{HCl}$ solutions since it has a relatively higher OCP than that of PTA HE6. While in a $10 \% \mathrm{H}_{2} \mathrm{SO}_{4}$ solution, HE6 might have better resistance to corrosion. However, the potentiodynamic testing cannot be substituted by OCP testing since it simulates much more complex situations than quasi-static OCP values reflect.

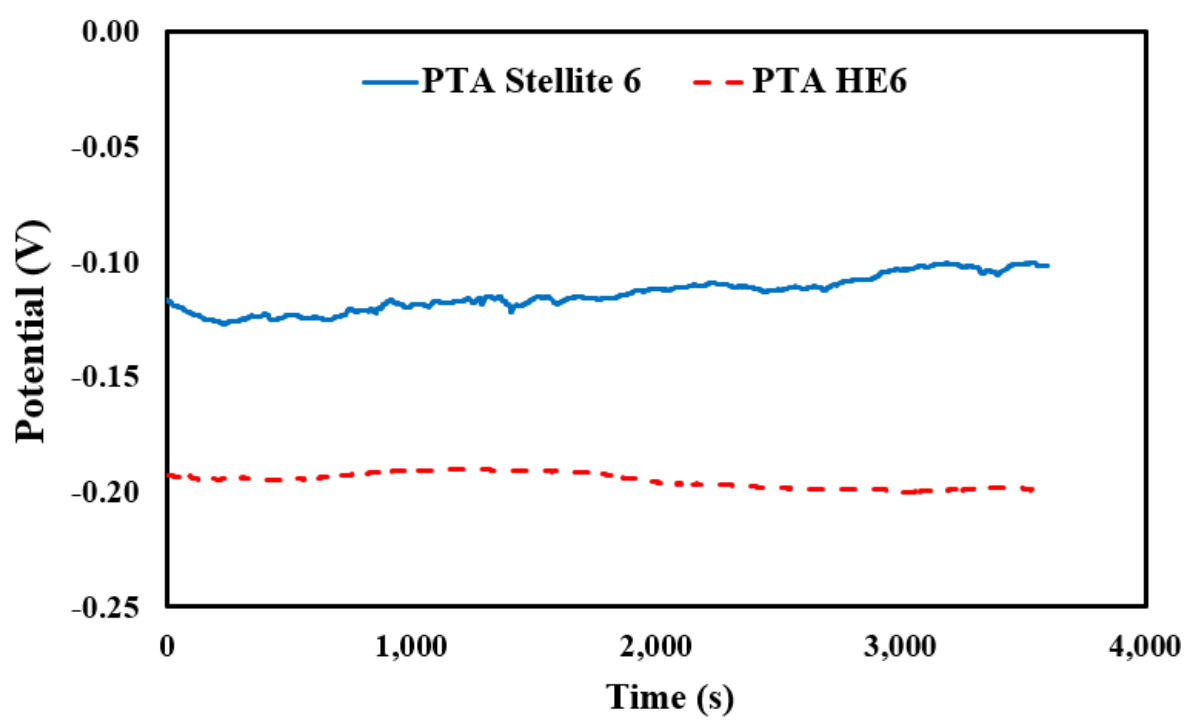

(a) 


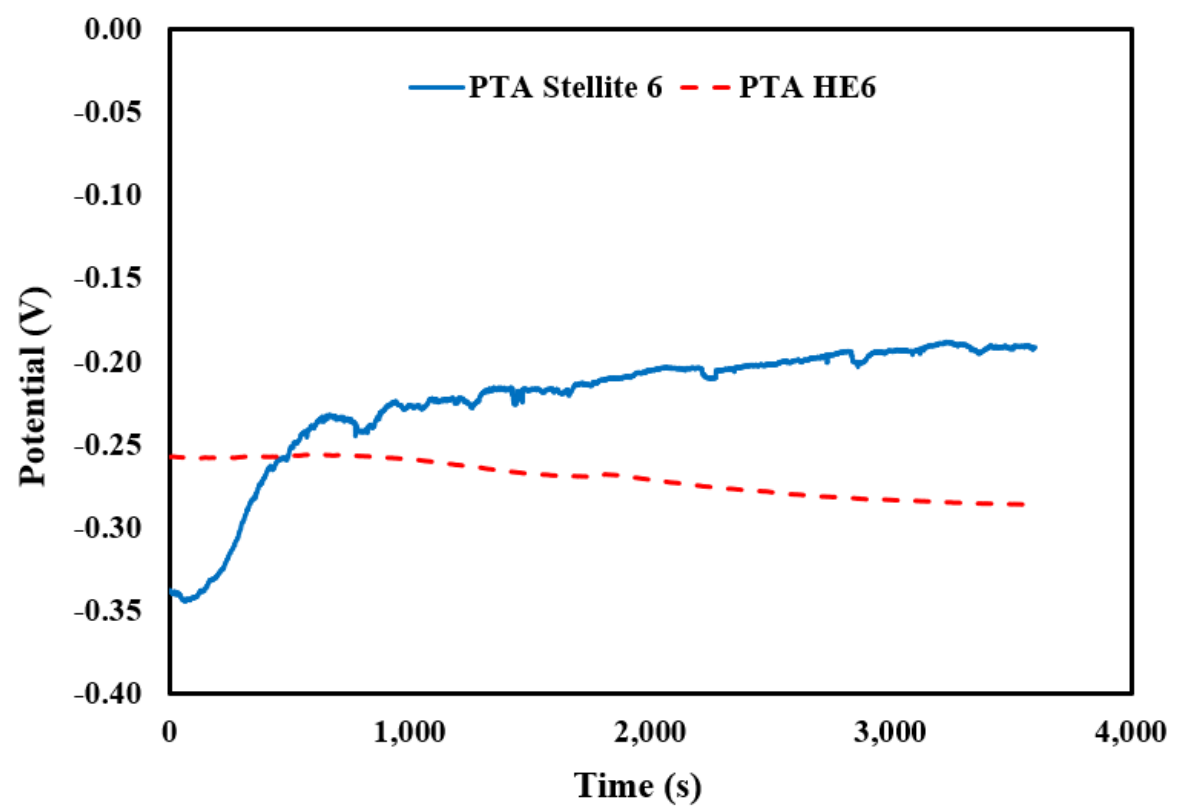

(b)

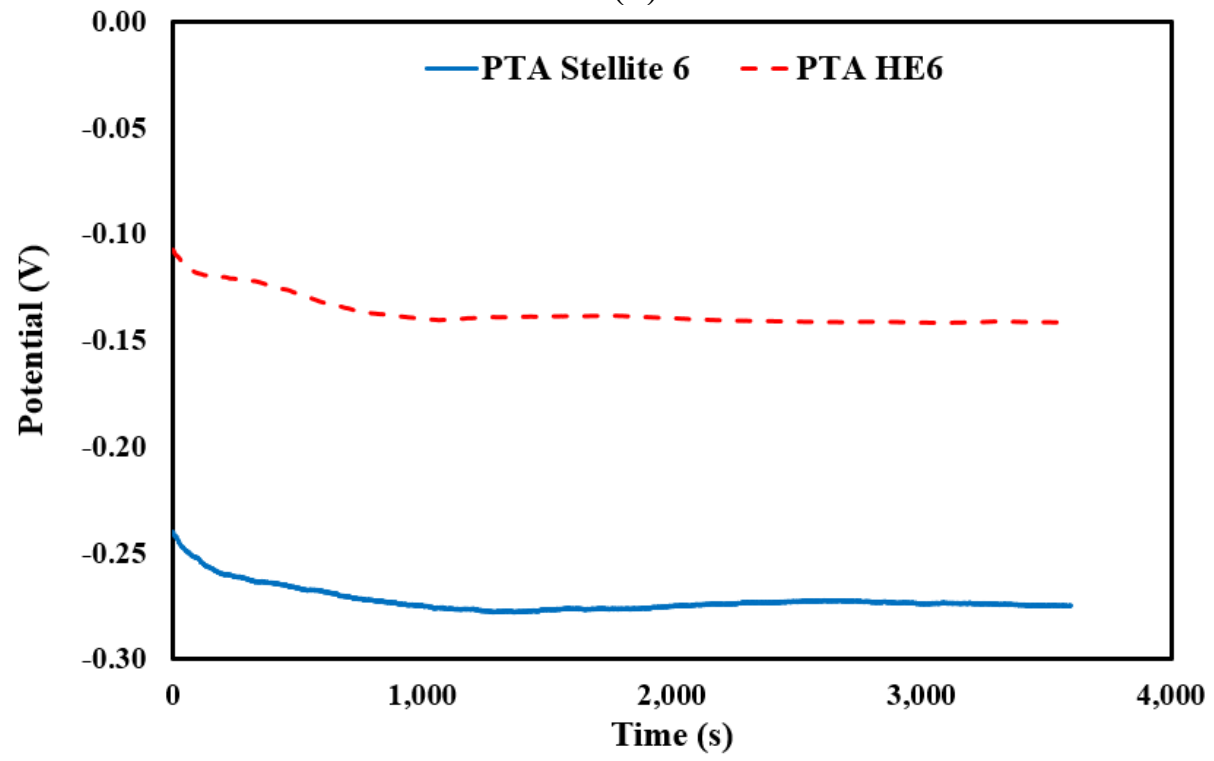

(c)

Figure 4-30: OCP curves of alloy specimens in (a) $3.5 \% \mathrm{NaCl}$, (b) $2 \% \mathrm{HCl}$, (c) $10 \% \mathrm{H}_{2} \mathrm{SO}_{4}$ solution. 


\subsubsection{EIS results and analyses}

Nyquist plot is often used to analyze the EIS data. As mentioned in Chapter 2, it can show the presence and integrity of resistive or passive films on the alloy surface. To better understand the plot, an equivalent circuit is often used to fit the impedance curve. The resistor $R$, inductor $L$ and capacitor $C$ are three common electrical elements equipped in the equivalent circuit. The impedance of the resistor is independent of the frequency; the impedance of the capacitor decreases when the frequency rises; the impedance of the inductor increases with the frequency rising. Additionally, the constant phase element (CPE) is employed as a substitute for the capacitor when the exposed surface is rough. The expressions of the electric elements are given in Equation 4.5 to 4.8.

For resistor:

$$
Z=R, Z^{\prime}=R, Z^{\prime \prime}=0
$$

For inductor:

$$
Z=i \omega L, Z^{\prime}=0, Z^{\prime \prime}=\omega L
$$

For capacitor:

$$
Z=\frac{1}{i \omega C}, Z^{\prime}=0, Z^{\prime \prime}=\frac{1}{\omega C}
$$

For CPE:

$$
\mathrm{Z}=\frac{1}{(i \omega)^{\alpha} Y_{0}}, Z^{\prime}=R, Z^{\prime \prime}=\frac{1}{\omega^{\alpha} Y_{0}}
$$

where $\omega$ refers to the frequency, $\alpha$ is the exponent varying from 0.5 to 1 and $Y_{0}$ represents the correction of capacity. When $\alpha$ equals 1 , the CPE is identical to the capacitor. In the real test, a CPE is usually selected since this element can better depict the actual circumstance of the test and the values of $\alpha$ and $Y_{0}$ can be calculated with the EIS software.

A simplified Randles circuit consists of the solution resistance $\left(R_{S}\right), \mathrm{CPE}$, and resistance of the protective film $\left(R_{f}\right)$ was employed in this study. This model is widely used as an initial point for other complex models [99] and is shown in Figure 4-31, which emphasizes the function of $R_{f}$, 
representing the resistance level of the surface protective layer. Three specimens of each alloy were tested in $3.5 \% \mathrm{NaCl}, 2 \% \mathrm{HCl}$ and $10 \% \mathrm{H}_{2} \mathrm{SO}_{4}$ solutions, respectively. The equivalent circuit in Figure 4-31 was chosen to fit the EIS data.

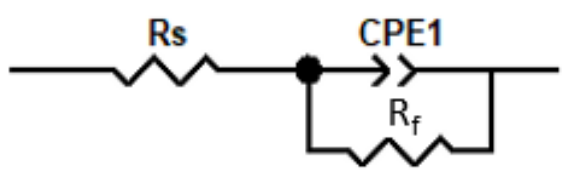

Figure 4-31: Simplified Randles circuit.

The EIS fitting results for PTA Stellite 6 and PTA HE6 are presented in the Nyquist plots shown in Figure 4-32 and Figure 4-33, respectively, with the fitted parameters are summarized in Table 4-3.

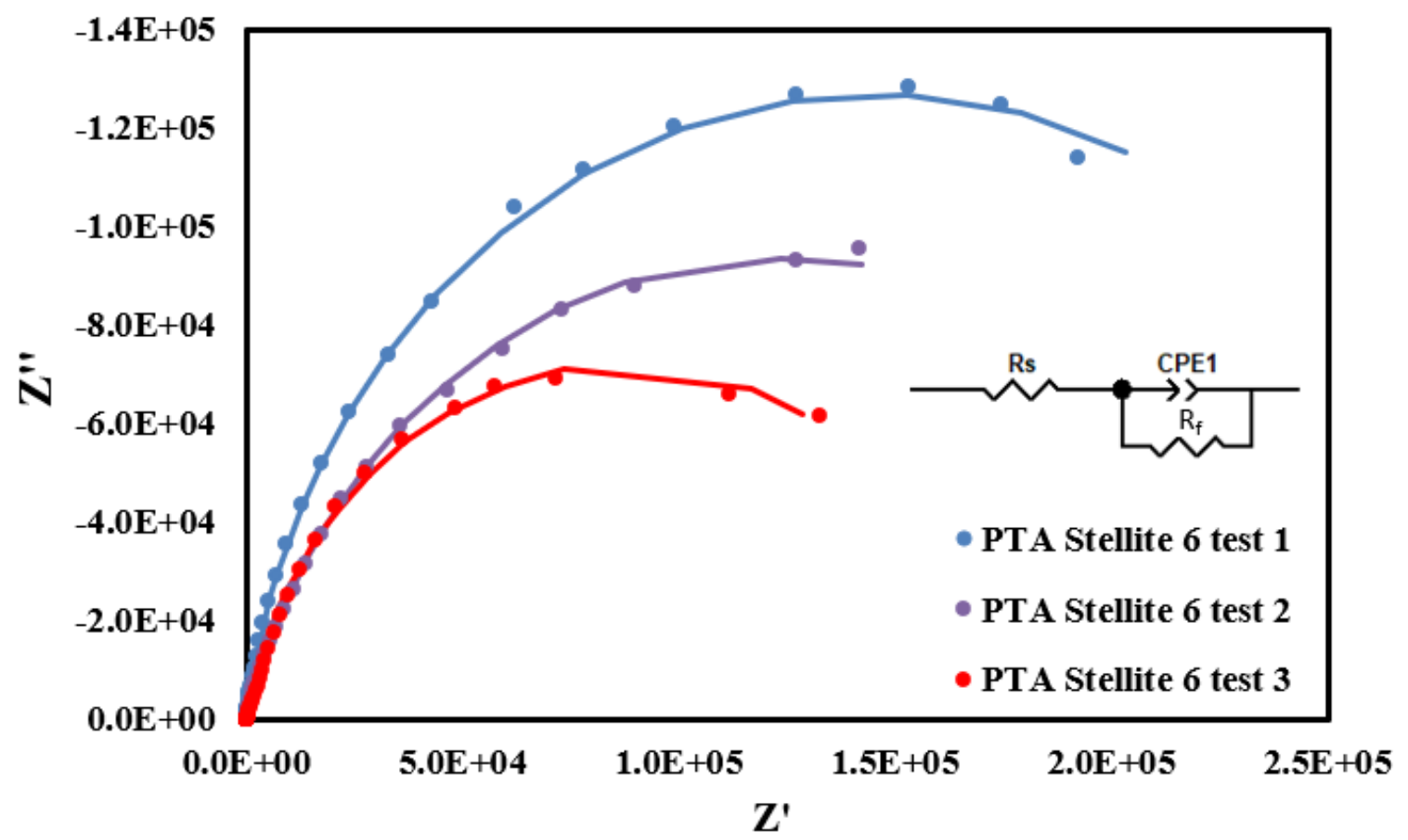

Figure 4-32: Nyquist plot of PTA Stellite 6 tested in 3.5\% NaCl solution. 


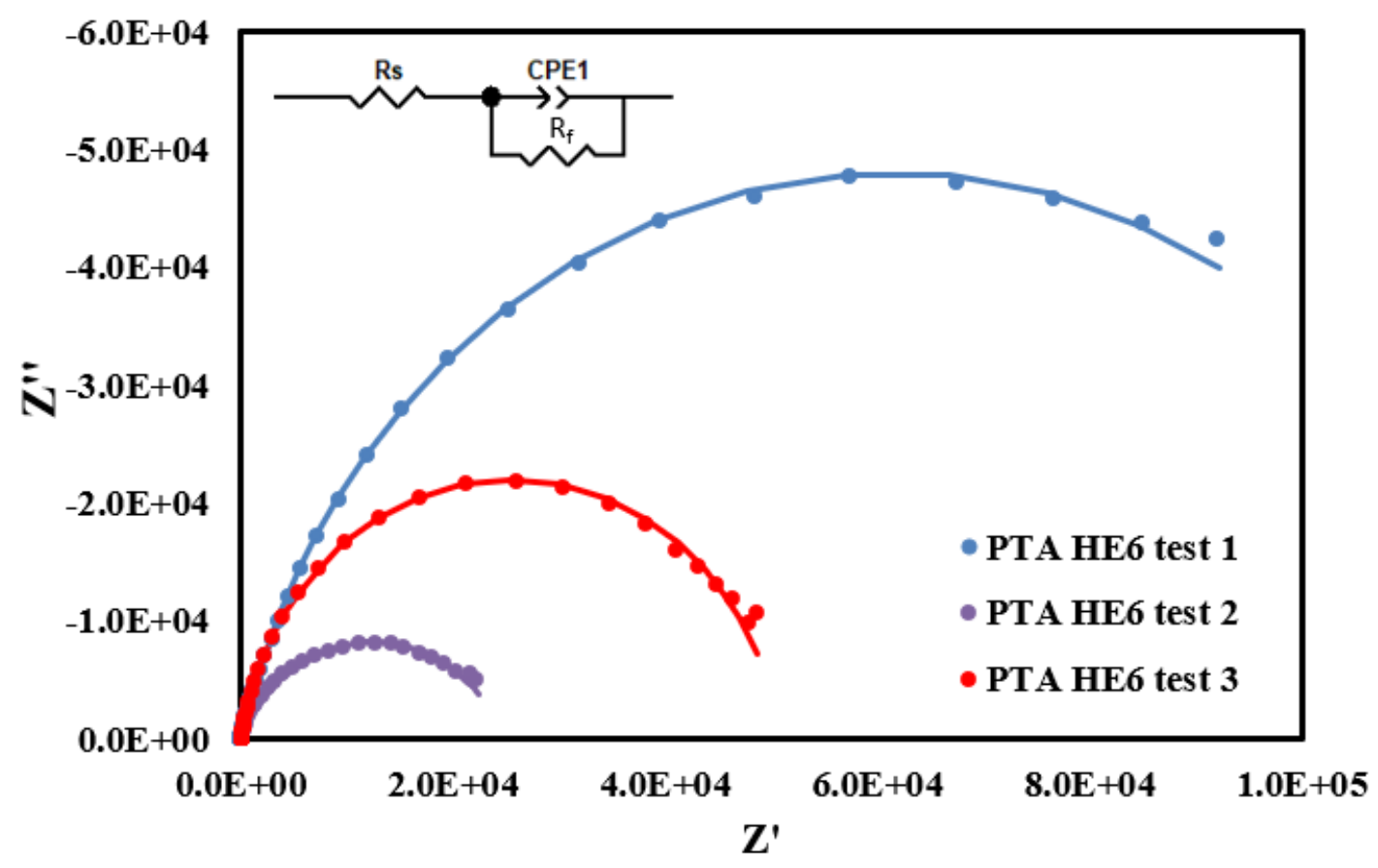

Figure 4-33: Nyquist plot of PTA HE6 tested in 3.5\% $\mathrm{NaCl}$ solution.

Table 4-3: EIS results of the tests in 3.5\% $\mathrm{NaCl}$ solution fitted from the equivalent circuit.

\begin{tabular}{|c|c|c|c|c|}
\hline Alloy & $\mathbf{1 0}^{-\mathbf{6}} \times \boldsymbol{Y}_{\mathbf{0}}\left(\boldsymbol{\Omega}^{-\mathbf{1}} \boldsymbol{S}^{\boldsymbol{\alpha}}\right)$ & $\boldsymbol{\alpha}$ & $\boldsymbol{R}_{\boldsymbol{f}}\left(\mathbf{k} \boldsymbol{\Omega} \cdot \mathbf{c m}^{\mathbf{2}}\right)$ & $\boldsymbol{R}_{\boldsymbol{s}}\left(\mathbf{k} \boldsymbol{\Omega} \cdot \mathbf{c m}^{\mathbf{2}}\right)$ \\
\hline PTA Stellite 6 & $31.63 \pm 3.57$ & $0.87 \pm 0.03$ & $2.37 \mathrm{E}+05 \pm 4.78 \mathrm{E}+04$ & $15.61 \pm 0.25$ \\
\hline PTA HE6 & $47.82 \pm 11.78$ & $0.83 \pm 0.07$ & $0.49 \mathrm{E}+05 \pm 1.95 \mathrm{E}+04$ & $15.65 \pm 0.53$ \\
\hline
\end{tabular}

From Figure 4-32 and Figure 4-33, for both PTA Stellite 6 and PTA HE6, the fitting curves (solid) coincide well with the testing data (point) indicating that the equivalent circuit is appropriate for the present EIS tests. However, apparent scatters in three repeated tests indicate the testing errors among the specimens of the same alloy grade. From Table 4-3, the values of $Y_{0}$, $\alpha$ and $R_{S}$ of Stellite 6 and HE6 are close, which reveals the similar corrosion mechanism of the alloys. However, the large difference in $R_{f}$ value implies that the corrosion resistance of the two alloys differs greatly and Stellite 6 is better in $3.5 \% \mathrm{NaCl}$ environment.

The EIS test results of PTA Stellite 6 and PTA HE6 in $\mathrm{HCl}$ solution are presented in Figure 4-34 and Figure 4-35, with the calculated parameters reported in Table 4-4. According to the values 
of the parameters, it can be suggested that Stellite 6 has better corrosion resistance in $2 \% \mathrm{HCl}$ environment because this alloy has lower capacitance $\left(Y_{0}\right)$ and larger resistance of the film $\left(R_{f}\right)$.

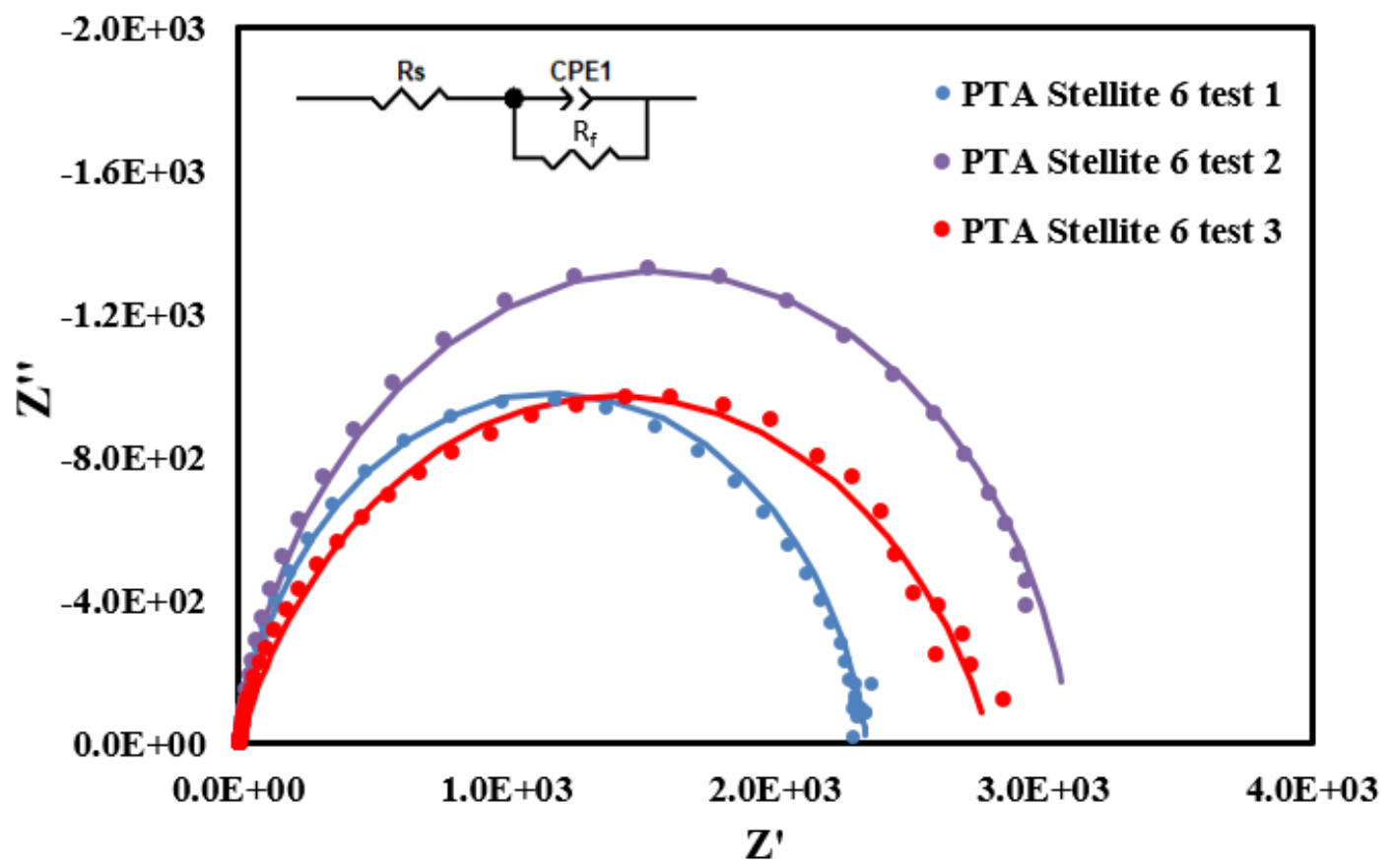

Figure 4-34: Nyquist plot of PTA Stellite 6 tested in 2\% $\mathrm{HCl}$ solution.

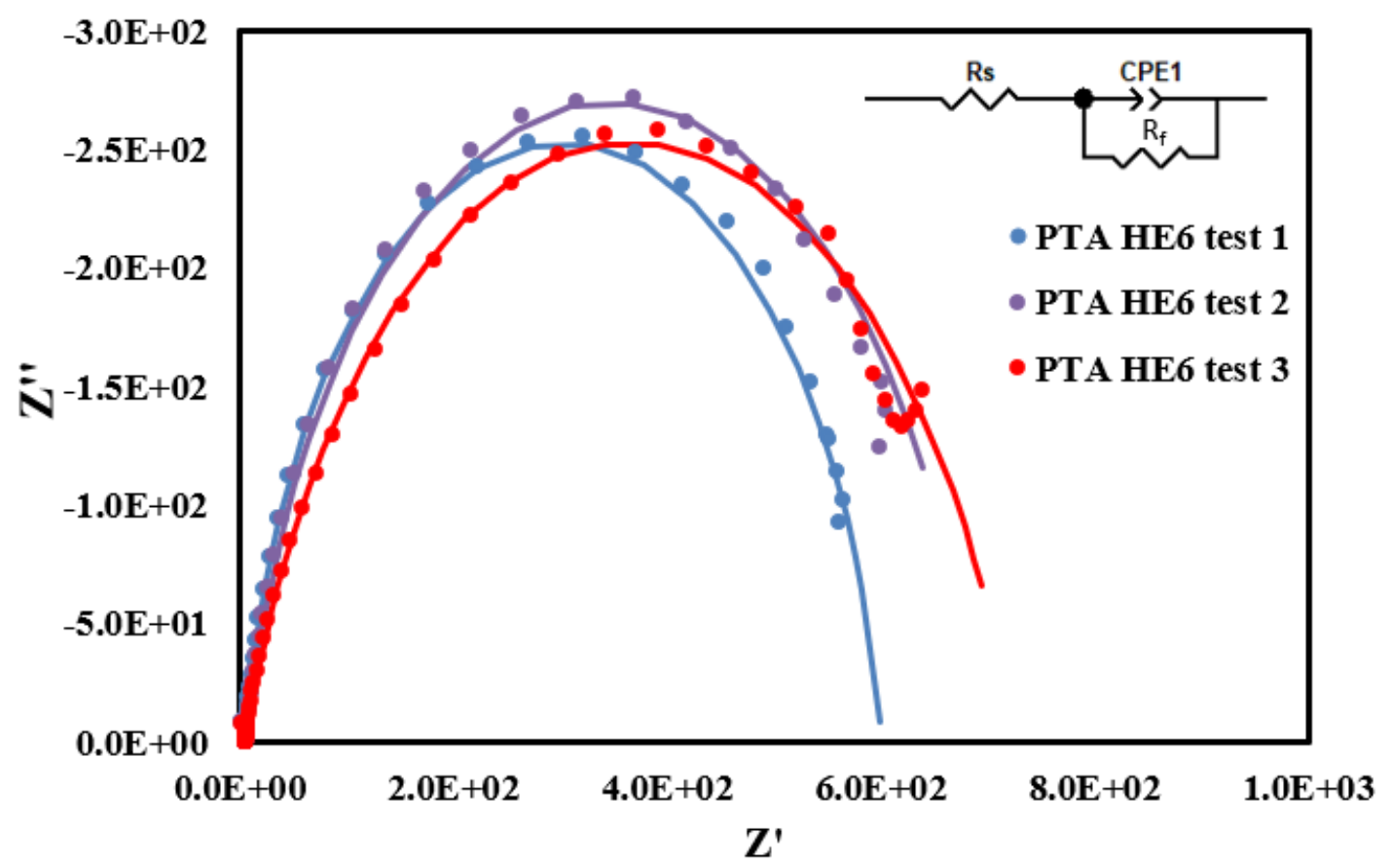

Figure 4-35: Nyquist plot of PTA HE6 tested in 2\% $\mathrm{HCl}$ solution. 
Table 4-4: EIS results of the tests in $2 \% \mathrm{HCl}$ solution fitted from the equivalent circuit.

\begin{tabular}{|c|c|c|c|c|}
\hline Alloy & $\mathbf{1 0}^{-\mathbf{6}} \times \boldsymbol{Y}_{\mathbf{0}}\left(\boldsymbol{\Omega}^{-\mathbf{1}} \boldsymbol{S}^{\boldsymbol{n}}\right)$ & $\boldsymbol{\alpha}$ & $\boldsymbol{R}_{\boldsymbol{f}}\left(\mathbf{k} \boldsymbol{\Omega} \cdot \mathbf{c m}^{\mathbf{2}}\right)$ & $\boldsymbol{R}_{\boldsymbol{s}}\left(\mathbf{k} \boldsymbol{\Omega} \cdot \mathbf{c m}^{\mathbf{2}}\right)$ \\
\hline PTA Stellite 6 & $66.59 \pm 23.03$ & $0.85 \pm 0.06$ & $2.75 \mathrm{E}+03 \pm 3.14 \mathrm{E}+02$ & $5.52 \pm 0.22$ \\
\hline PTA HE6 & $333.10 \pm 134.80$ & $0.84 \pm 0.05$ & $0.67 \mathrm{E}+03 \pm 0.53 \mathrm{E}+02$ & $5.09 \pm 0.13$ \\
\hline
\end{tabular}

The EIS test results of PTA Stellite 6 and PTA HE6 in $\mathrm{H}_{2} \mathrm{SO}_{4}$ solution are presented in Figure 4-34 and Figure 4-35, with the calculated parameters reported in Table 4-5. A similar conclusion can be drawn that Stellite 6 performed better in $\mathrm{H}_{2} \mathrm{SO}_{4}$ solution because of larger $R_{f}$ value.

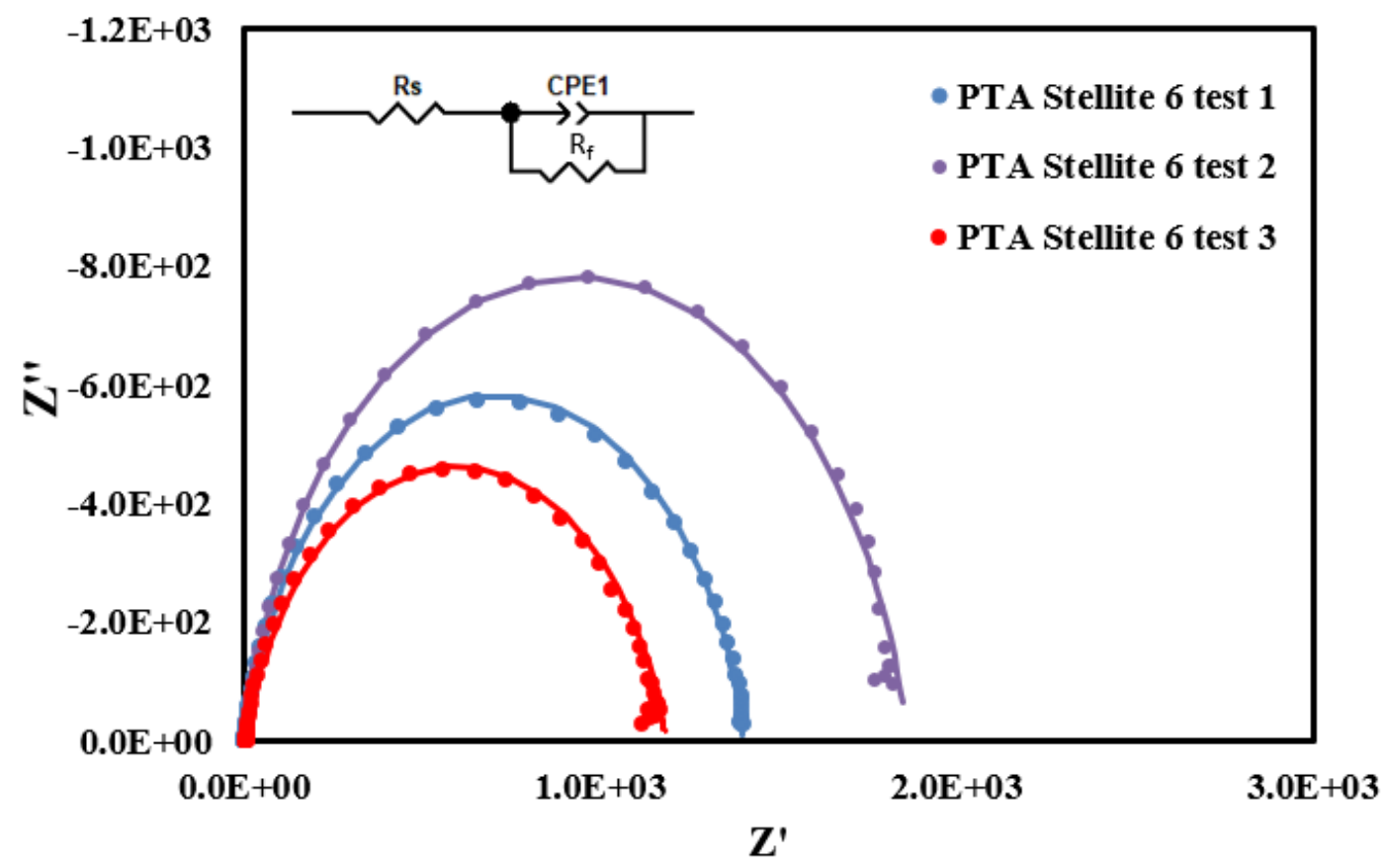

Figure 4-36: Nyquist plot of PTA Stellite 6 tested in $10 \% \mathrm{H}_{2} \mathrm{SO}_{4}$ solution. 


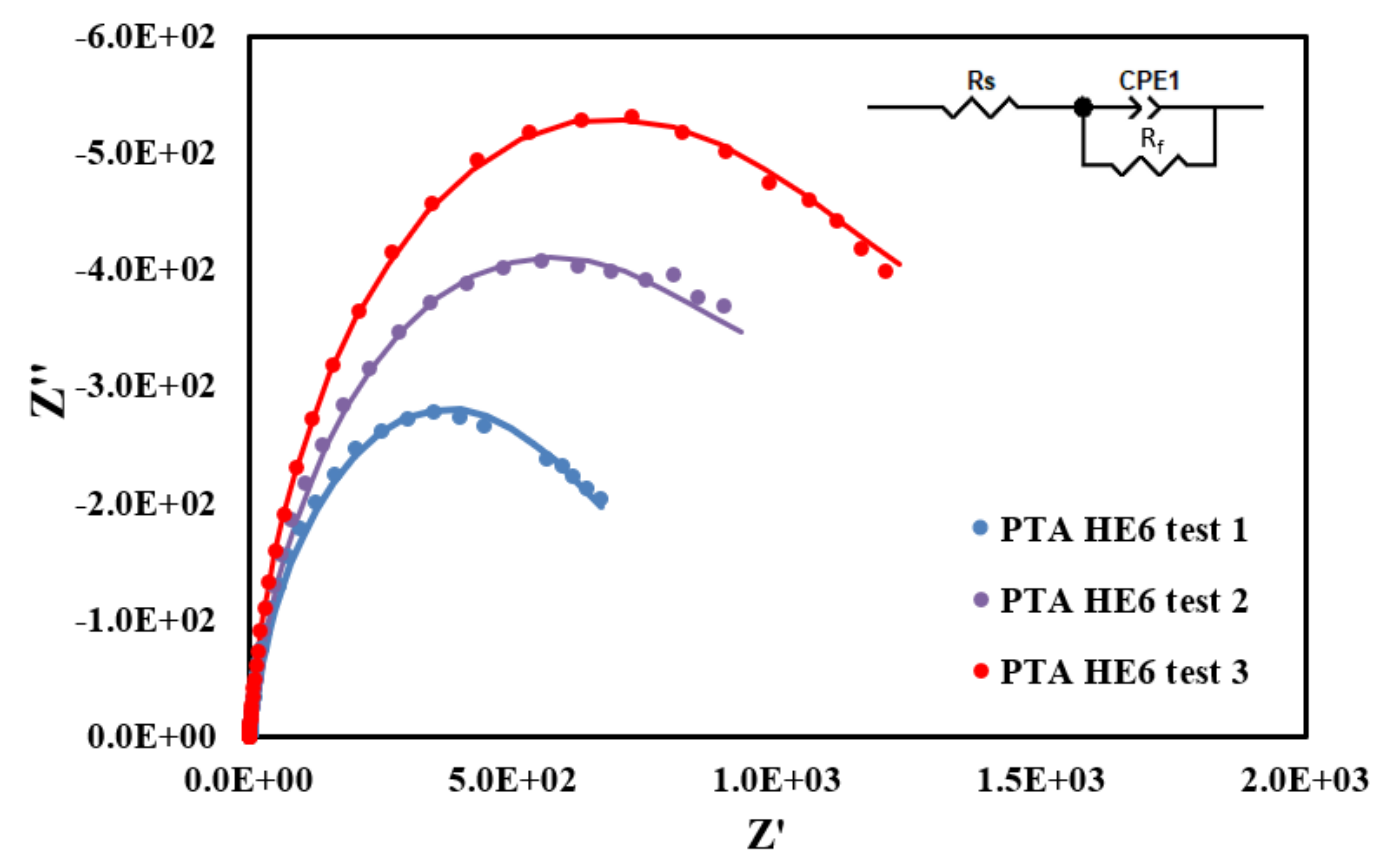

Figure 4-37: Nyquist plot of PTA HE6 tested in $10 \% \mathrm{H}_{2} \mathrm{SO}_{4}$ solution.

Table 4-5: EIS results of the tests in $10 \% \mathrm{H}_{2} \mathrm{SO}_{4}$ solution fitted from the equivalent circuit.

\begin{tabular}{|c|c|c|c|c|}
\hline Alloy & $\mathbf{1 0}^{-6} \times \boldsymbol{Y}_{\mathbf{0}}\left(\boldsymbol{\Omega}^{-\mathbf{1}} \boldsymbol{S}^{\boldsymbol{n}}\right)$ & $\boldsymbol{\alpha}$ & $\boldsymbol{R}_{\boldsymbol{f}}\left(\mathbf{k} \boldsymbol{\Omega} \cdot \mathbf{c m}^{\mathbf{2}}\right)$ & $\boldsymbol{R}_{\boldsymbol{s}}\left(\mathbf{k} \boldsymbol{\Omega} \cdot \mathbf{c m}^{\mathbf{2}}\right)$ \\
\hline PTA Stellite 6 & $60.90 \pm 7.85$ & $0.87 \pm 0.02$ & $1.48 \mathrm{E}+03 \pm 2.81 \mathrm{E}+02$ & $3.79 \pm 0.91$ \\
\hline PTA HE6 & $450.10 \pm 108.01$ & $0.87 \pm 0.03$ & $0.75 \mathrm{E}+03 \pm 1.08 \mathrm{E}+02$ & $3.05 \pm 0.81$ \\
\hline
\end{tabular}

For comparison, the $R_{f}$ values of the alloys tested in the three media are illustrated on the logarithmic scale in Figure 4-38. It is clear that both alloys were more resistant to $3.5 \% \mathrm{NaCl}$ solution, but they both behaved similarly in the other solutions. Between the two alloys, Stellite 6 performed better in all solutions. Moreover, EIS results are generally very sensitive to test conditions, for example, specimen surfaces may affect the electrochemical process even if the surfaces are polished with the same sandpapers. In this study, $\mathrm{pH}$ variation of the electrolyte was not monitored during the EIS test, which may contribute to the scatter of the results. 


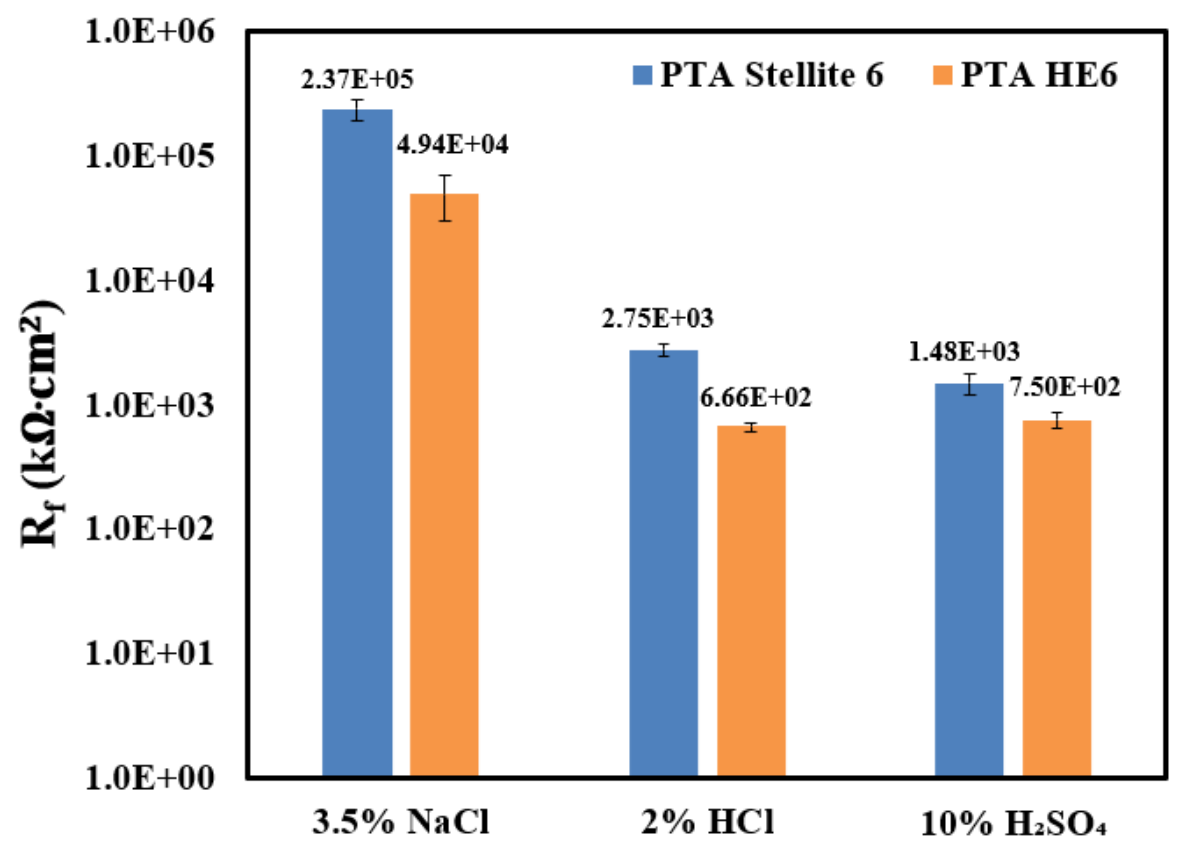

Figure 4-38: Comparison of $R_{f}$ value from EIS tests.

\subsubsection{Cyclic polarization curves}

Cyclic polarization test was conducted on PTA Stellite 6 and PTA HE6 specimens in 3.5\% $\mathrm{NaCl}, 2 \% \mathrm{HCl}$ and $10 \% \mathrm{H}_{2} \mathrm{SO}_{4}$ solutions. Three repeated tests were performed on each alloy in each solution to verify the repeatability of the results. For the polarization curve analysis, the Xaxis refers to the logarithmic value of current density which is the absolute value of the ratio of current to the exposed surface area (a circle with a diameter of $10 \mathrm{~mm}$, as shown in Figure 4-39). The Y-axis refers to a relative scale that represents applied potential voltage vs. SCE. 


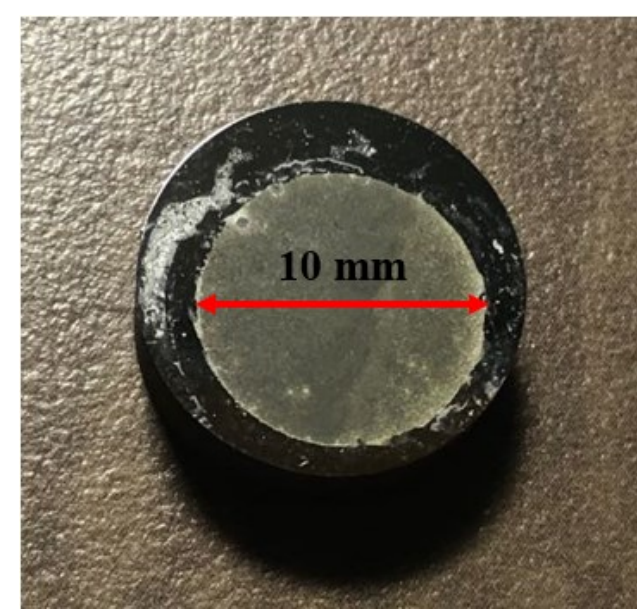

Figure 4-39: Corrosion test specimen showing the exposed area to the electrolyte.

All curves of the cyclic polarization tests were analyzed using CView ${ }^{\mathrm{TM}}$ software. The corrosion parameters such as corrosion potential $\left(E_{\text {corr }}\right)$, current density $\left(I_{c o r r}\right)$, anodic Tafel slope $\left(\beta_{a}\right)$, cathodic Tafel slope $\left(\beta_{c}\right)$ and corrosion rate $(\mathrm{CR})$ were obtained directly from the software. Then polarization resistance $\left(R_{p}\right)$ can be calculated through the Tafel Equation [94].

The cyclic polarization curves from three tests of PTA Stellite 6 and PTA HE6 alloys in $3.5 \% \mathrm{NaCl}$ solution are presented in Figure 4-40 and Figure 4-41, respectively. In general, the cyclic polarization test exhibited good repeatability, in particular, for HE6 specimens. Certainly, some deviations are observed on the curves of both alloys, especially in the reverse scan on Stellite 6 specimens, as shown in Figure 4-40, which implies that these specimens have different resistance to localized corrosion. Generally, backward scans are more scattered than the forward scans, because the specimen surface has already been corroded during the forward scanning thus leaving the surfaces in various conditions. According to the cyclic polarization curves in Figure 4-40 and Figure 4-41, despite the scatters in backward scans, good repeatability is verified for both alloys. The average values of corrosion parameters obtained from the curves are summarized in Table 4-6. 


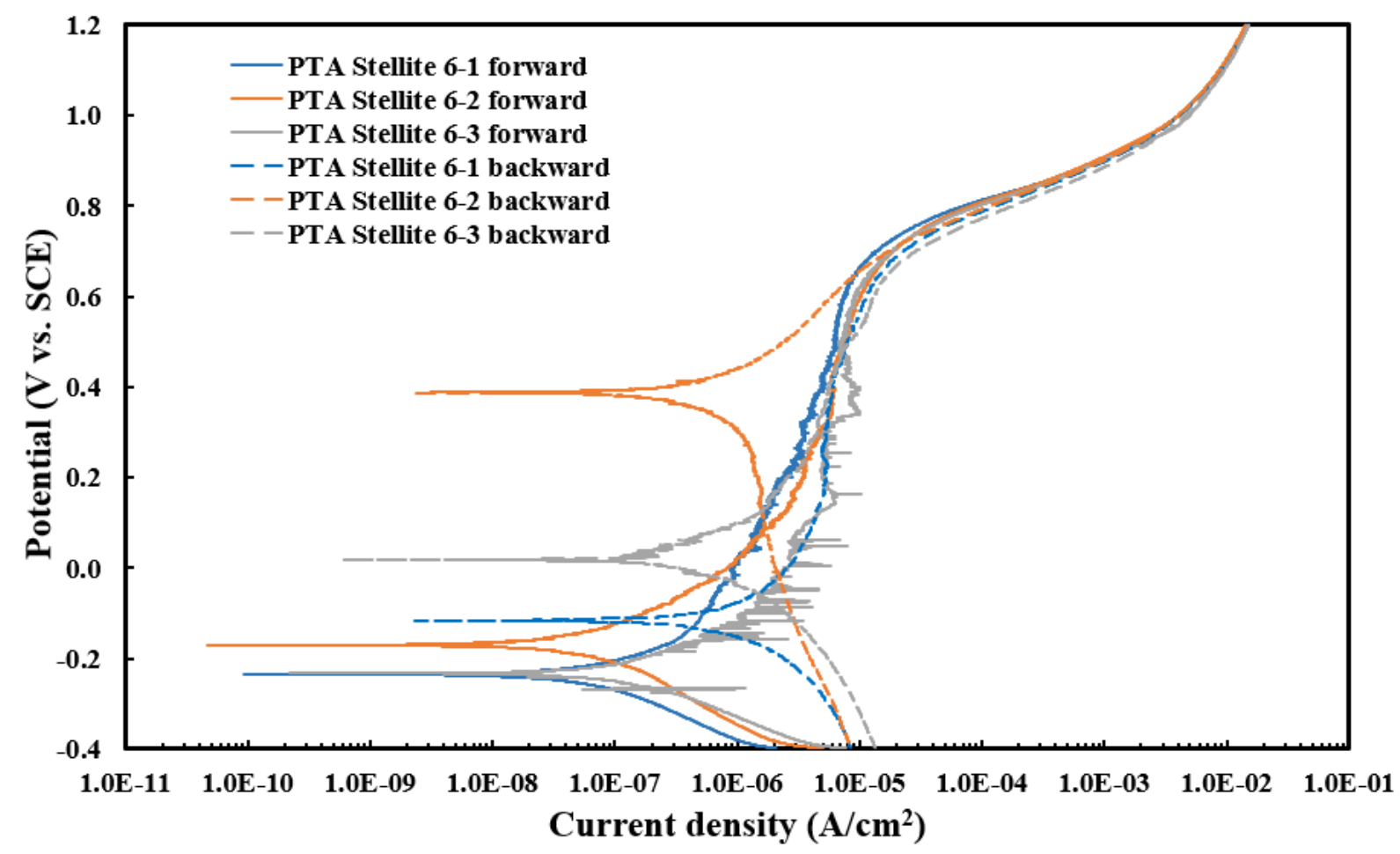

Figure 4-40: Cyclic polarization curves of PTA Stellite 6 alloy tested in $3.5 \% \mathrm{NaCl}$.

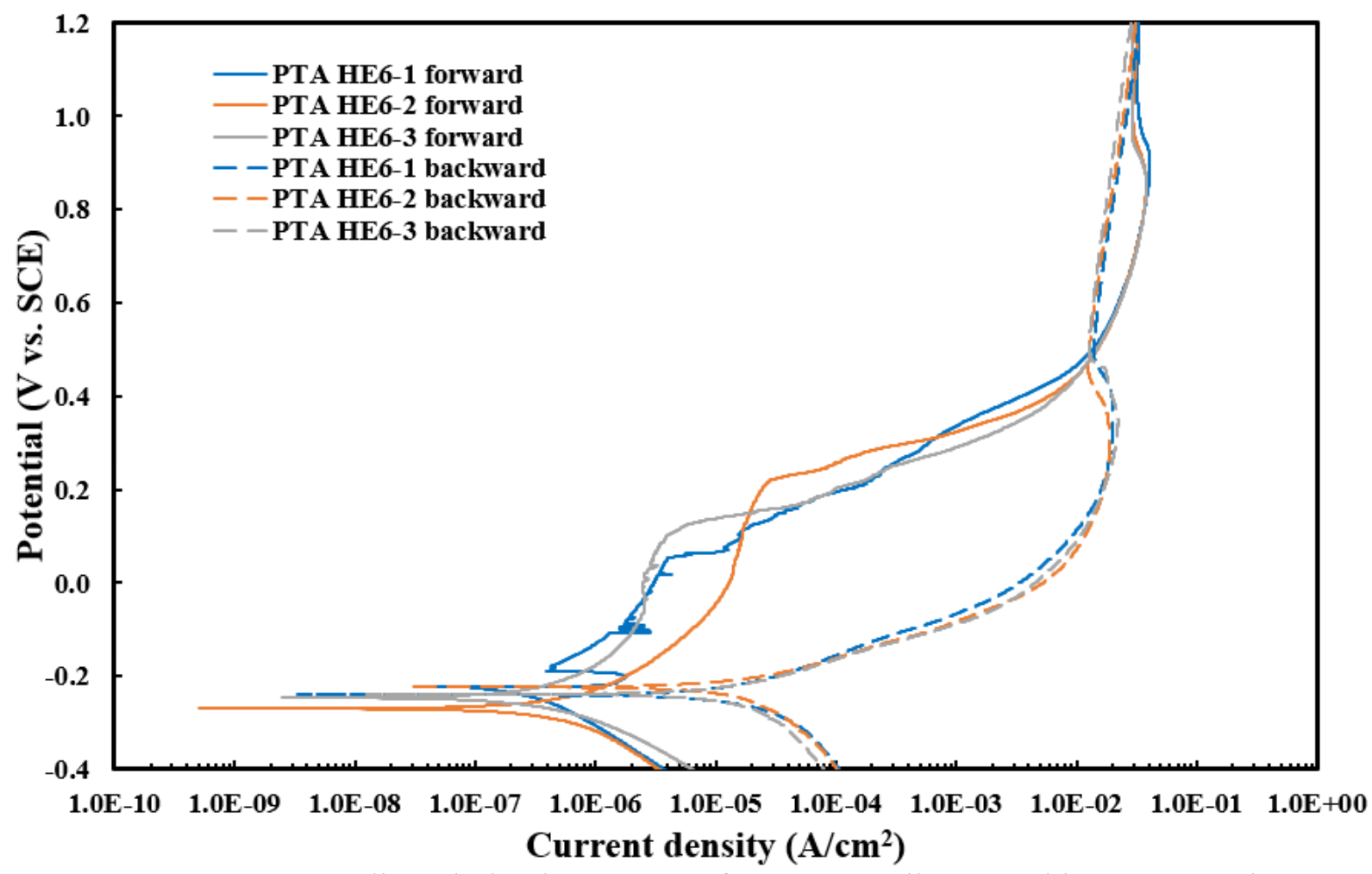

Figure 4-41: Cyclic polarization curves of PTA HE6 alloy tested in 3.5\% NaCl. 
For comparison, one cyclic polarization curve of each alloy is plotted together in Figure 4-42. It is shown that PTA Stellite 6 has higher corrosion potential $(-0.21 \mathrm{~V})$ than PTA HE6 $(-0.25$ V) and a lower corrosion rate (7.39E-08 vs. $6.66 \mathrm{E}-07 \mathrm{~mm} / \mathrm{yr})$. This indicates that PTA Stellite 6 has better general corrosion resistance in 3.5\% $\mathrm{NaCl}$ environment. For both alloys, as the potential was continuously raised, the slope dramatically increased, leading to the formation of a passive region. A large passive region suggests the formation of oxide film on the specimen surface that has a great potential to slow the corrosion rate. Generally speaking, the larger the passive region, the better anti-corrosion ability the alloy possesses. From Figure 4-42, PTA Stellite 6 has a larger passive region in the potential range from 0.0 to $0.6 \mathrm{~V}$ vs. SCE, while PTA HE6 has the range from 0.0 to $0.2 \mathrm{~V}$ vs. SCE. When the scanning potential exceeded the upper limit of the passive zone, the current started to rise again, as a result of the oxide film broken down, which can speed up the corrosion. It can also be inferred that the protective film was destroyed at that turning point. PTA Stellite 6 tended to obtain a second passive region because the slope became large again around 1.0 V. However, for PTA HE6, a second passive region occurred when the scanning voltage exceeded $0.4 \mathrm{~V}$, as marked in Figure 4-42, suggesting the repair of the oxide film on the specimen surface during the corrosion process.

Combining the forward and reverse scans constitute a hysteresis loop. If the reverse curve lays on the left side of the forward one, it is defined as electropositive, otherwise, it is electronegative. Electropositive infers better resistance to localized (pitting) corrosion due to lower current density during the reverse scan.

As shown in Figure 4-42, PTA Stellite 6 demonstrates a small degree of electronegative behavior during the whole reverse scanning, which indicates that the alloy has little pitting 
resistance in $3.5 \% \mathrm{NaCl}$, while the PTA HE6 exhibits electropositive at the beginning of the reverse scan, indicating good anti-pitting ability. However, it is also noted that the reverse scan curve of PTA HE6 later turns to a large degree of electronegative, meaning that severe corrosion had happened on this alloy.

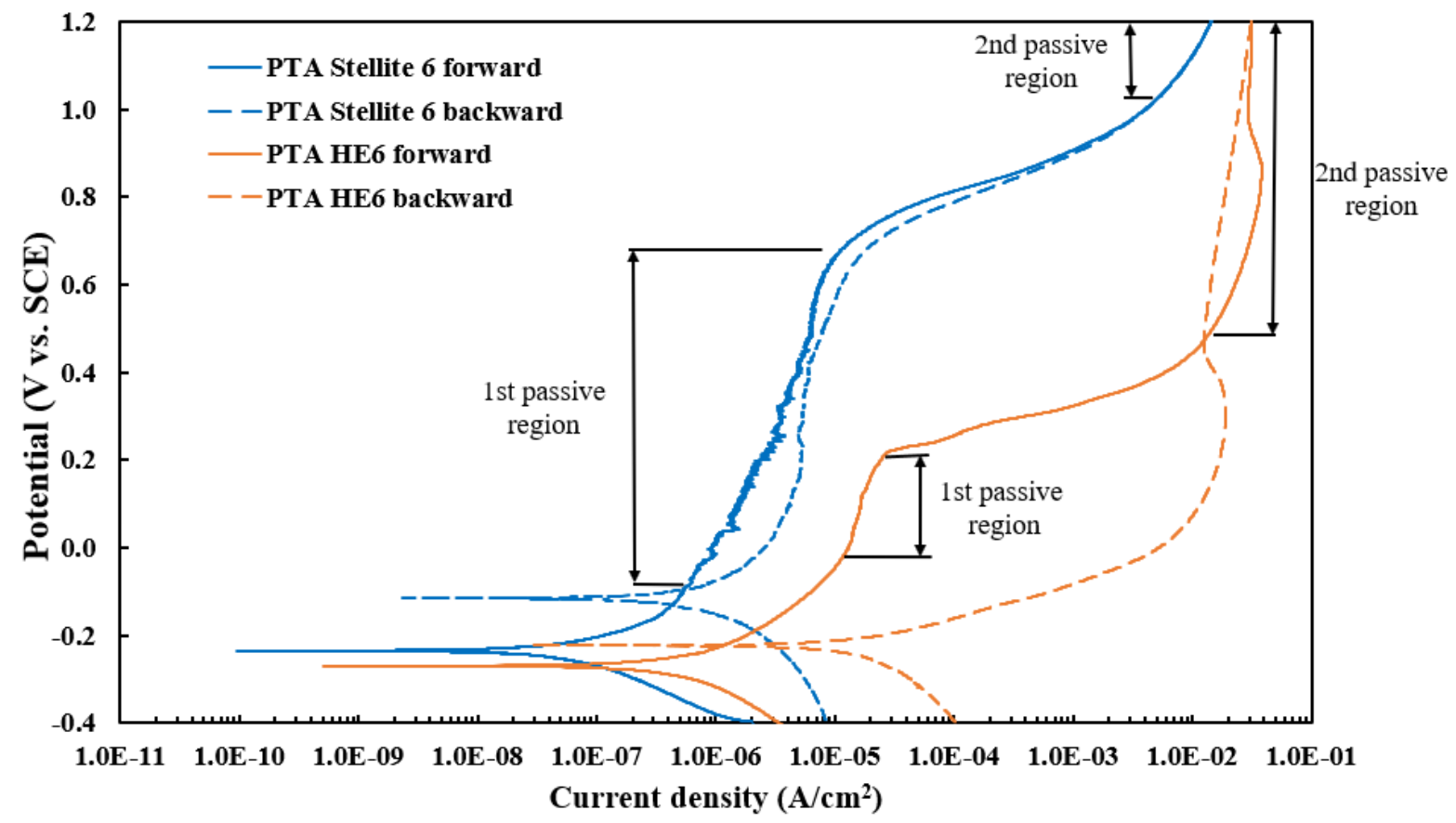

Figure 4-42: Comparison of polarization behavior between PTA Stellite 6 and PTA HE6 in $3.5 \% \mathrm{NaCl}$ solution.

Table 4-6: Summary of polarization test results of the alloys in $3.5 \% \mathrm{NaCl}$ solution.

\begin{tabular}{|c|c|c|c|c|c|c|c|c|}
\hline \multirow{2}{*}{ Specimen } & \multicolumn{3}{|c|}{ PTA Stellite 6 } & \multicolumn{4}{c|}{ PTA HE6 } \\
\cline { 2 - 9 } & Test 1 & Test 2 & Test 3 & Average & Test 1 & Test 2 & Test 3 & Average \\
\hline $\begin{array}{c}\boldsymbol{E}_{\boldsymbol{c o r r}} \\
(\mathbf{V ~ v s . S C E})\end{array}$ & -0.24 & -0.17 & -0.23 & $-0.21 \pm 0.03$ & -0.23 & -0.27 & -0.25 & $-0.25 \pm 0.02$ \\
\hline $\begin{array}{c}\boldsymbol{I}_{\boldsymbol{c o r r}} \\
\left(\boldsymbol{\mu} \mathbf{A} / \mathbf{c m}^{2}\right)\end{array}$ & 0.05 & 0.07 & 0.06 & $0.06 \pm 0.01$ & 0.51 & 0.39 & 0.81 & $0.57 \pm 0.18$ \\
\hline $\begin{array}{c}\mathbf{C R} \\
(\mathbf{m m} / \mathbf{y r})\end{array}$ & $5.97 \mathrm{E}-08$ & $9.04 \mathrm{E}-08$ & $7.14 \mathrm{E}-08$ & $\begin{array}{c}7.39 \mathrm{E}-08 \\
\pm 1.27 \mathrm{E}-08\end{array}$ & $5.98 \mathrm{E}-07$ & $4.56 \mathrm{E}-07$ & $9.44 \mathrm{E}-07$ & $\begin{array}{c}6.66 \mathrm{E}-07 \\
\pm 2.05 \mathrm{E}-07\end{array}$ \\
\hline $\begin{array}{c}\boldsymbol{R}_{\boldsymbol{p}} \\
\left(\mathbf{k} \boldsymbol{\Omega} \cdot \mathbf{c m}^{\mathbf{2}}\right)\end{array}$ & 360.93 & 423.40 & 209.71 & $331.35 \pm 89.71$ & 68.24 & 58.65 & 59.49 & $62.13 \pm 4.34$ \\
\hline
\end{tabular}


The cyclic polarization curves of PTA Stellite 6 and PTA HE6 alloy tested in 2\% $\mathrm{HCl}$ solution are presented in Figure 4-43 and Figure 4-44, respectively. As the same, the repeatability from three tests is verified for both alloys.

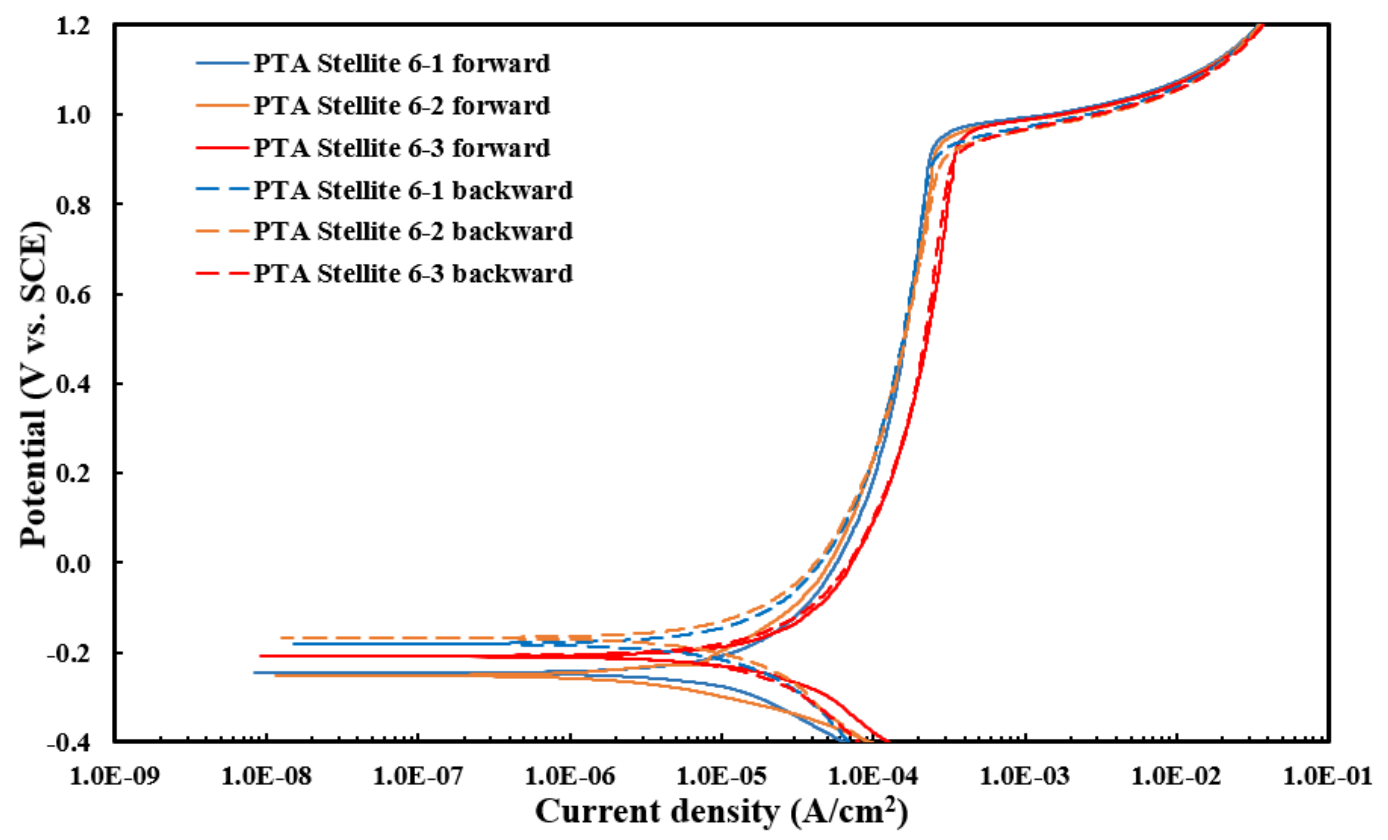

Figure 4-43: Cyclic polarization curves of PTA Stellite 6 alloy tested in 2\% $\mathrm{HCl}$ solution.

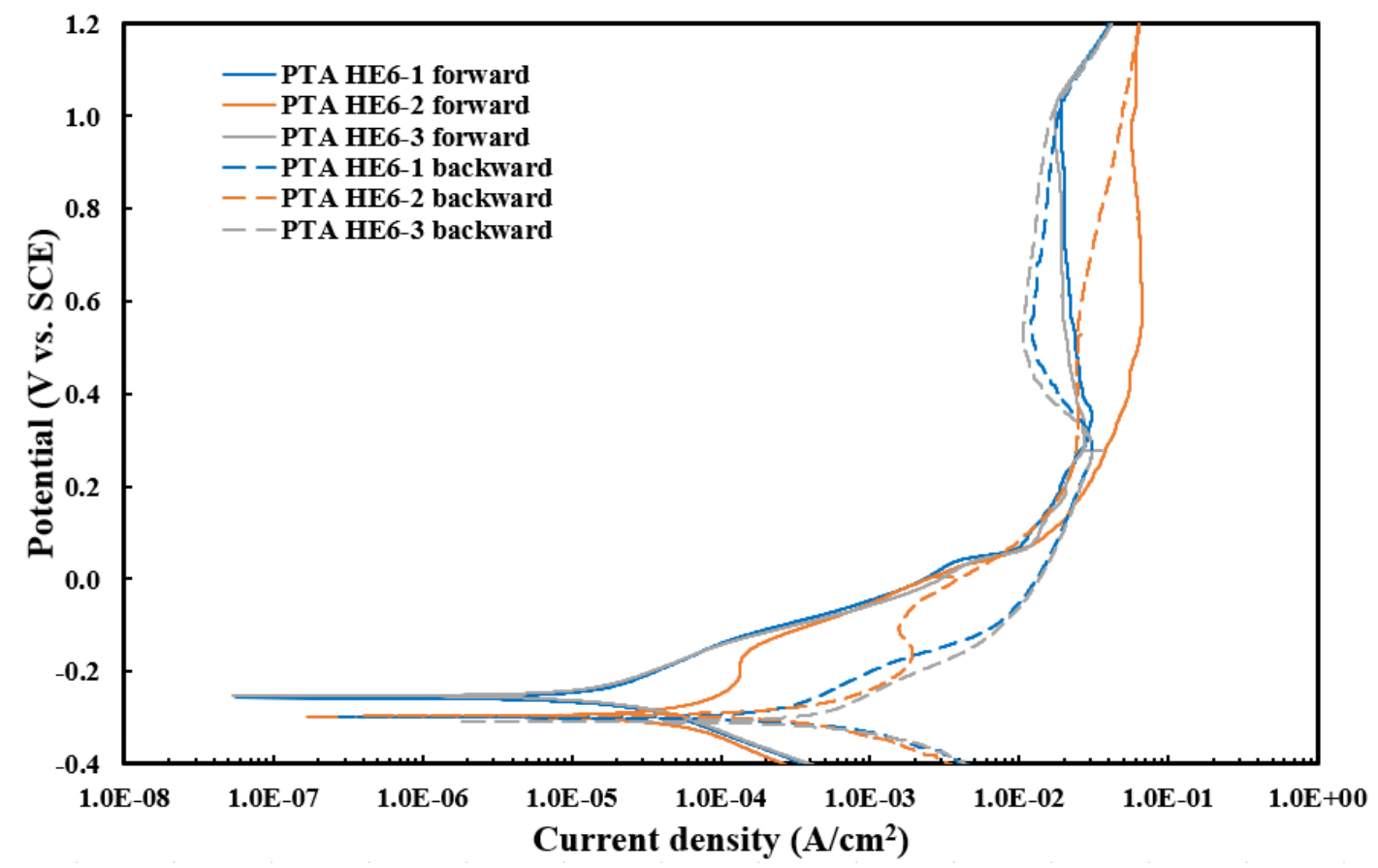

Figure 4-44: Cyclic polarization curves of PTA HE6 alloy tested in 2\% $\mathrm{HCl}$ solution. 
Similarly, one curve of each alloy was chosen to present together in Figure 4-45 for comparison and the values of corrosion parameters are summarized in Table 4-7. As shown, PTA Stellite 6 has higher $E_{\text {corr }}(-0.22 \mathrm{~V})$ in comparison with PTA HE6 $(-0.27 \mathrm{~V})$, along with lower $I_{\text {corr }}\left(20.31\right.$ vs. $\left.79.94 \mu \mathrm{A} / \mathrm{cm}^{2}\right)$ and smaller value of CR (2.56E-05 vs. $\left.9.34 \mathrm{E}-05 \mathrm{~mm} / \mathrm{yr}\right)$, which suggests that PTA Stellite 6 generally performed better in $2 \% \mathrm{HCl}$ environment. For both alloys, there is an apparent passive region on cyclic polarization curves, indicating the oxidation ability of the alloys. The passive regions of the two alloys are comparable because of the similar potential ranges.

Regarding the hysteresis loop, PTA Stellite 6 shows a little electronegative behavior at the beginning of the reverse scan and turns to slightly electropositive later on. It is suggested that Stellite 6 has some ability to resist pitting corrosion in 2\% HCl. For PTA HE6 the backward polarization curve shows electropositive in the initial stage of the reverse scan, which suggests relatively good pitting corrosion resistance of this alloy, but the polarization behavior converts to electronegative $0.3 \mathrm{~V}$, which is regarded as pitting resistance decay. 


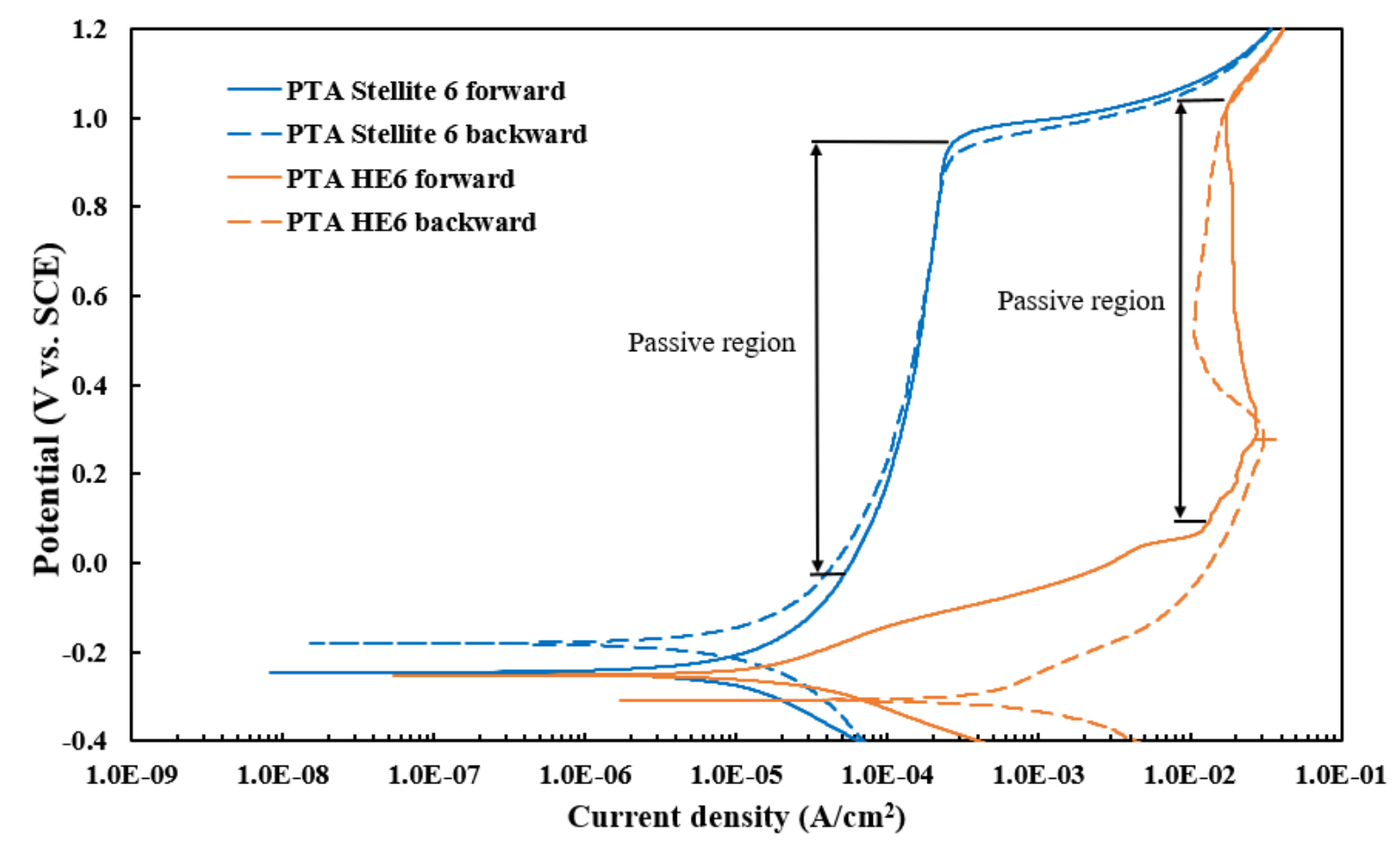

Figure 4-45: Comparison of cyclic polarization behavior between PTA Stellite 6 and PTA HE6 in $2 \% \mathrm{HCl}$ solution.

Table 4-7: Summary of polarization test results of the alloys in $2 \% \mathrm{HCl}$ solution.

\begin{tabular}{|c|c|c|c|c|c|c|c|c|}
\hline \multirow{2}{*}{ Specimen } & \multicolumn{4}{|c|}{ PTA Stellite 6} & \multicolumn{4}{|c|}{ PTA HE6 } \\
\hline & Test 1 & Test 2 & \begin{tabular}{|l|} 
Test 3 \\
\end{tabular} & Average & Test 1 & Test 2 & \begin{tabular}{|l|} 
Test 3 \\
\end{tabular} & Average \\
\hline $\begin{array}{c}\boldsymbol{E}_{\text {corr }} \\
(\mathbf{V} \text { vs. } S C E)\end{array}$ & -0.25 & -0.19 & -0.21 & $-0.22 \pm 0.02$ & -0.26 & -0.30 & -0.25 & $-0.27 \pm 0.02$ \\
\hline $\begin{array}{c}I_{\text {corr }} \\
\left(\boldsymbol{\mu} \mathbf{A} / \mathbf{c m}^{2}\right)\end{array}$ & 15.31 & 15.25 & 30.38 & $20.31 \pm 7.12$ & 28.94 & 178.90 & 31.99 & $79.94 \pm 69.98$ \\
\hline $\begin{array}{c}\text { CR } \\
(\mathrm{mm} / \mathbf{y r})\end{array}$ & $1.93 \mathrm{E}-05$ & $1.92 \mathrm{E}-05$ & 3.83E-05 & $\begin{array}{c}2.56 \mathrm{E}-05 \\
\pm 8.99 \mathrm{E}-06 \\
\end{array}$ & $3.38 \mathrm{E}-07$ & 1.09E-04 & $3.74 \mathrm{E}-05$ & $\begin{array}{c}9.34 \mathrm{E}-05 \\
\pm 3.47 \mathrm{E}-05\end{array}$ \\
\hline $\begin{array}{c}\boldsymbol{R}_{\boldsymbol{p}} \\
\left(\mathbf{k} \boldsymbol{\Omega} \cdot \mathbf{c m}^{2}\right)\end{array}$ & 3.67 & 3.94 & 2.18 & $3.26 \pm 0.77$ & 1.15 & 0.44 & 1.16 & $0.92 \pm 0.34$ \\
\hline
\end{tabular}

The cyclic polarization curves of PTA Stellite 6 and PTA HE6 alloy tested in $10 \% \mathrm{H}_{2} \mathrm{SO}_{4}$ solution are given in Figure 4-46 and Figure 4-47, respectively. In Figure 4-46 for PTA Stellite 6, Test 1 and Test 3 repeated each other well, but Test 2 demonstrated three peaks in the forward scan. The first peak of Test 2 coincided with the other two tests, which means that the three specimens of Stellite 6 had similar corrosion resistance initially. However, after the corrosion had 
happened, there might be a deviation in the degree of corrosion on the surface between the specimens. Similar scenario is observed on PTA HE6, there are also multiple peaks occurring in the forward scan, showing that both alloys resist a strongly oxidizing environment in a similar manner by generating protective oxide films repeatedly during the polarization test.

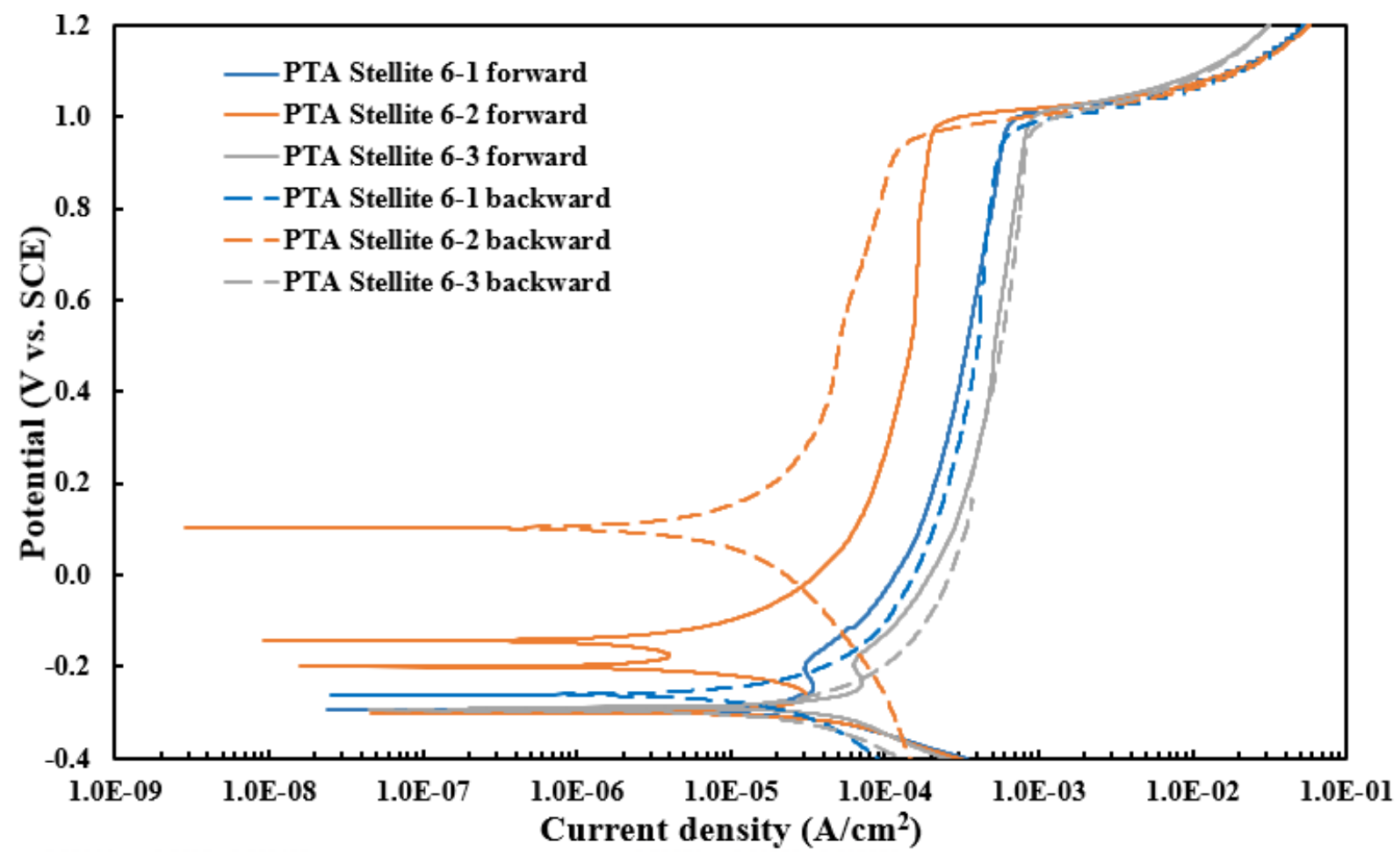

Figure 4-46: Cyclic polarization curves of PTA Stellite 6 alloy tested in $10 \% \mathrm{H}_{2} \mathrm{SO}_{4}$. 


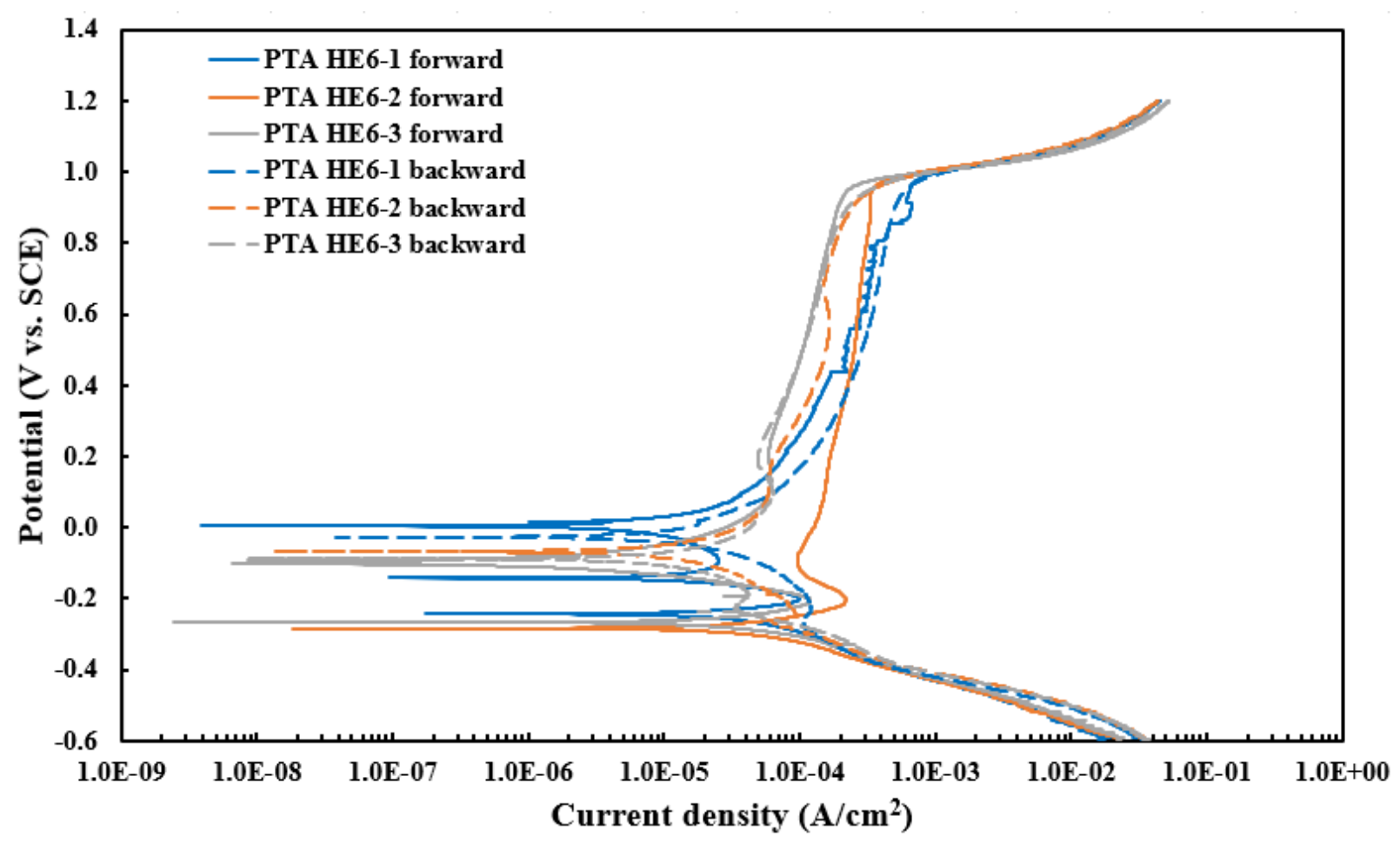

Figure 4-47: Cyclic polarization curves of PTA HE6 alloy tested in $10 \% \mathrm{H}_{2} \mathrm{SO}_{4}$.

One curve of each alloy was taken to plot together in Figure 4-48 for comparison and the corrosion parameters obtained from all polarization curves are reported in Table 4-8. In general, the two alloys exhibited little difference in $10 \% \mathrm{H}_{2} \mathrm{SO}_{4}$ solution because their polarization curves nearly overlap. On the backward curves, PTA HE6 shows electropositive behavior suggesting better pitting corrosion resistance. 


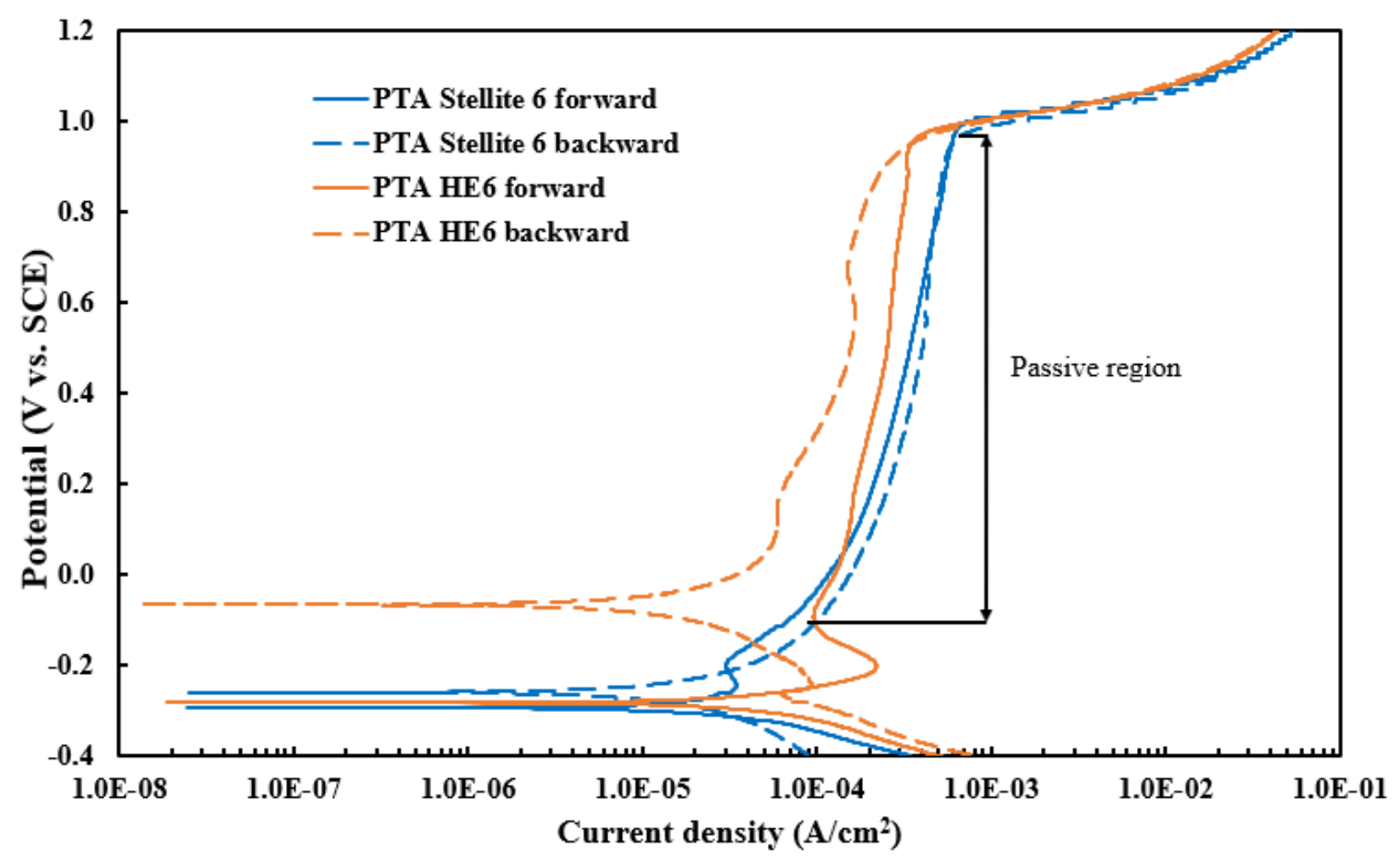

Figure 4-48: Comparison of cyclic polarization behavior between PTA Stellite 6 and PTA HE6 in $10 \% \mathrm{H}_{2} \mathrm{SO}_{4}$ solution.

Table 4-8: Summary of polarization test results of the alloys in $10 \% \mathrm{H}_{2} \mathrm{SO}_{4}$ solution.

\begin{tabular}{|c|c|c|c|c|c|c|c|c|}
\hline \multirow{2}{*}{ Specimen } & \multicolumn{4}{|c|}{ PTA Stellite 6} & \multicolumn{4}{|c|}{ PTA HE6 } \\
\hline & Test 1 & Test 2 & Test 3 & Average & Test 1 & Test 2 & \begin{tabular}{|l|} 
Test 3 \\
\end{tabular} & Average \\
\hline $\begin{array}{c}\boldsymbol{E}_{\text {corr }} \\
(\mathbf{V} \text { vS. SCE })\end{array}$ & -0.30 & -0.30 & -0.26 & $-0.28 \pm 0.02$ & -0.24 & -0.28 & -0.25 & $-0.26 \pm 0.02$ \\
\hline $\begin{array}{c}I_{\text {corr }} \\
\left(\boldsymbol{\mu A} / \mathbf{c m}^{2}\right)\end{array}$ & 43.88 & 26.40 & 32.76 & $34.35 \pm 7.22$ & 54.64 & 68.26 & 39.81 & $54.24 \pm 11.62$ \\
\hline $\begin{array}{c}\text { CR } \\
(\mathrm{mm} / \mathrm{yr})\end{array}$ & $5.54 \mathrm{E}-05$ & $3.33 \mathrm{E}-05$ & 7.92E-05 & $\begin{array}{c}5.60 \mathrm{E}-05 \\
\pm 1.87 \mathrm{E}-05 \\
\end{array}$ & 6.39E-05 & 7.98E-05 & $4.65 \mathrm{E}-05$ & $\begin{array}{c}6.34 \mathrm{E}-05 \\
\pm 1.36 \mathrm{E}-05 \\
\end{array}$ \\
\hline $\begin{array}{c}R_{p} \\
\left(\mathbf{k} \boldsymbol{\Omega} \cdot \mathbf{c m}^{2}\right)\end{array}$ & 0.74 & 0.58 & 1.45 & $0.92 \pm 0.38$ & 0.45 & 0.41 & 0.38 & $0.41 \pm 0.03$ \\
\hline
\end{tabular}

The overall corrosion rates of PTA Stellite 6 and PTA HE6 in the polarization tests are illustrated on the logarithm scale in Figure 4-49. In summary, PTA Stellite 6 has a lower corrosion rate than PTA HE6 in all tested corrosive environments. The lowest corrosion rates for both alloys 
were found in $3.5 \% \mathrm{NaCl}$ solution. For Stellite 6, the highest corrosion rate occurred in $10 \% \mathrm{H}_{2} \mathrm{SO}_{4}$ environment, while for $\mathrm{HE} 6$ the highest corrosion rate occurred in $2 \% \mathrm{HCl}$ solution.

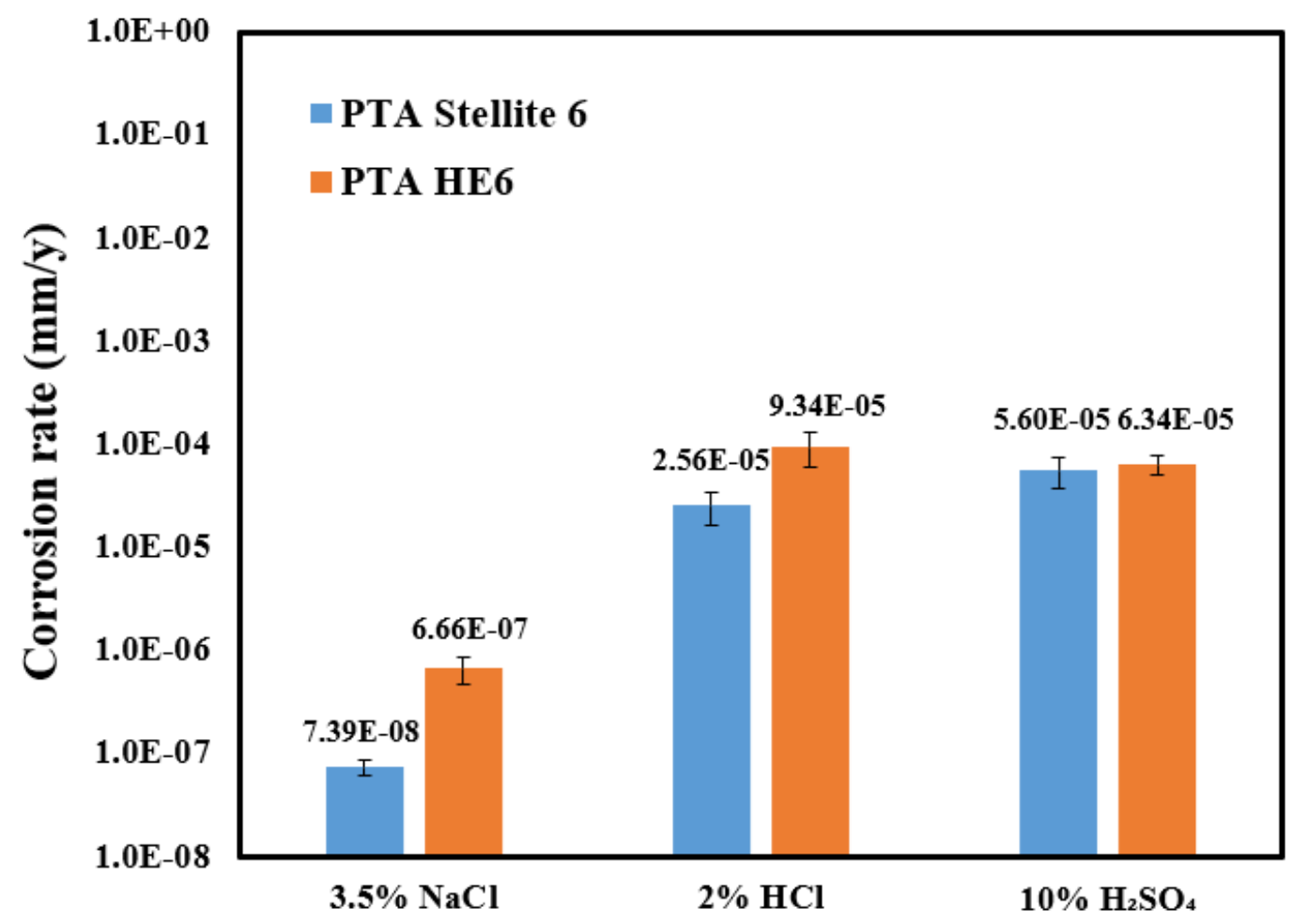

Figure 4-49: Corrosion rates of tested alloys in polarization tests.

\subsubsection{Immersion tests}

Immersion test was performed on PTA Stellite 6 and PTA HE6 alloys in hydrochloric acid $(5 \% \mathrm{HCl})$ and sulfuric acid $\left(10 \% \mathrm{H}_{2} \mathrm{SO}_{4}\right)$ for 24 and 72 hours, respectively, at room temperature.

\subsubsection{Specimen and solution preparation}

The surfaces of the PTA Stellite 6 and PTA HE6 specimens for immersion test were polished using a set of sandpapers to meet the requirements of ASTM Standard G31 - 12a [100], followed by ultrasonic cleaning for 15 minutes. Then, the mass (g) and geometrical dimensions of each specimen were measured. 
In the immersion test, $5 \% \mathrm{HCl}$ and $10 \% \mathrm{H}_{2} \mathrm{SO}_{4}$ were selected as corrosive media. $500 \mathrm{ml}$ solution was prepared for each test to assure sufficient reaction between the specimen and the agent. $57 \mathrm{ml} 37 \% \mathrm{HCl}$ or $30 \mathrm{ml} 98 \% \mathrm{H}_{2} \mathrm{SO}_{4}$ was diluted with distilled water to reach the set concentration.

\subsubsection{Experiment setup and procedure}

Following ASTM Standard G31 - 12a [100], a simplified setup of immersion test was employed in this study. A beaker covered with parafilm was used to execute the immersion test, as shown in Figure 4-50. The specimen was held on a plastic clip, as shown in Figure 4-51, to ensure the maximum surface exposure. Three specimens of each alloy were immersed in each batch, and the average value of the mass loss was obtained.

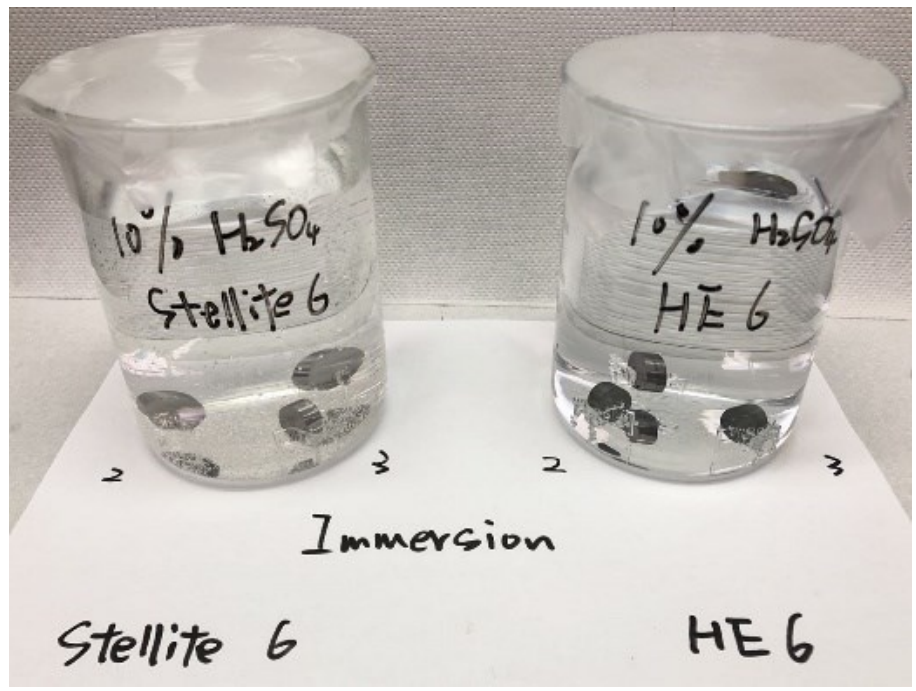

Figure 4-50: Immersion test setup. 


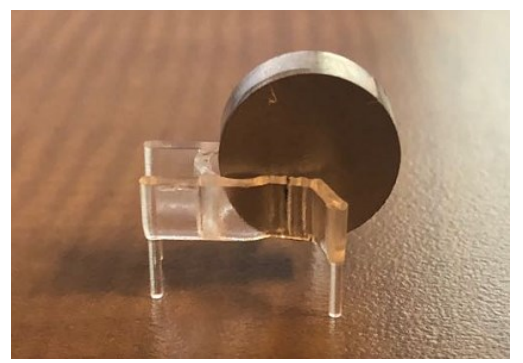

Figure 4-51: Alloy specimen held on a plastic clip.

After each immersion test, the specimen was taken out from the beaker and cleaned with an ultrasonic cleaner for 15 minutes, followed by hot air drying. The mass of each tested specimen was measured again to obtain the mass change. Guided by ASTM Standard G31 - 12a, the corrosion rate of the specimen can be calculated by the following formula [100]:

$$
\text { Corrosion rate }=(K \times W) /(A \times T \times D)
$$

where $W$ is the mass loss in $\mathrm{g}, A$ refers to the exposed area in $\mathrm{cm}^{2}, T$ is immersion time in hour and $D$ is the density in $\mathrm{g} / \mathrm{cm}^{3} . K$ is a unit conversion constant. In this study, $K$ is $8.76 \times 10^{4}$ to obtain the unit of corrosion rate in $\mathrm{mm} / \mathrm{y}$.

\subsubsection{Immersion test results}

The immersion test results of PTA Stellite 6 and PTA HE6 at room temperature are reported in Table 4-9 and Figure 4-52. In 5\% $\mathrm{HCl}$ solution, Stellite 6 had a higher corrosion rate than HE6 for both 24 and 72-hour testing durations, which means that Stellite 6 is less resistant to reducing environment. Additionally, the corrosion rate of Stellite 6 for 72 hour testing duration was almost twice higher than that for 24 hour duration, while HE6 maintained a similar level. Moreover, considering the larger data scattering of Stellite 6 for 72 hour testing duration, it can be inferred that some of the PTA Stellite 6 overlays may contain internal weld defects that were 
deleterious to the corrosion performance of the specimen. In $10 \% \mathrm{H}_{2} \mathrm{SO}_{4}$ solution, Stellite 6 behaved well for 24 hour testing duration because of little corrosion loss, but with the test proceeding, the corrosion rate rose drastically — more than eighty times higher at 72 hours. For HE6, the corrosion rate did not change much with testing duration, and overall HE6 performed better than Stellite 6 in $10 \% \mathrm{H}_{2} \mathrm{SO}_{4}$ solution according to the corrosion rate values. In summary, PTA HE6 had good weld quality due to its stable corrosion rate and very small standard deviation of corrosion rate in both reducing and oxidizing environments.

Table 4-9: Corrosion rates ( $\mathrm{mm} / \mathrm{y})$ of the alloys in immersion tests.

\begin{tabular}{|c|c|c|c|c|c|}
\hline & \multicolumn{2}{|c|}{$5 \% \mathrm{HCl}$} & \multicolumn{2}{|c|}{$10 \% \mathrm{H}_{2} \mathrm{SO}_{4}$} \\
\hline & & Test & Ave. & Test & Ave. \\
\hline \multirow{6}{*}{$\begin{array}{c}\text { PTA } \\
\text { Stellite } 6\end{array}$} & \multirow{3}{*}{$24 \mathrm{~h}$} & 2.16 & \multirow{3}{*}{$2.13 \pm 0.05$} & 0.02 & \multirow{3}{*}{$0.02 \pm 0.003$} \\
\hline & & 2.06 & & 0.02 & \\
\hline & & 2.18 & & 0.02 & \\
\hline & \multirow{3}{*}{$72 \mathrm{~h}$} & 3.11 & \multirow{3}{*}{$3.86 \pm 1.02$} & 1.59 & \multirow{3}{*}{$1.67 \pm 0.09$} \\
\hline & & 5.29 & & 1.63 & \\
\hline & & 3.16 & & 1.81 & \\
\hline \multirow{6}{*}{$\begin{array}{l}\text { PTA } \\
\text { HE6 }\end{array}$} & \multirow{3}{*}{$24 \mathrm{~h}$} & 0.37 & \multirow{3}{*}{$0.39 \pm 0.03$} & 0.71 & \multirow{3}{*}{$0.61 \pm 0.11$} \\
\hline & & 0.43 & & 0.68 & \\
\hline & & 0.37 & & 0.45 & \\
\hline & \multirow{3}{*}{$72 \mathrm{~h}$} & 0.32 & \multirow{3}{*}{$0.28 \pm 0.03$} & 0.72 & \multirow{3}{*}{$0.72 \pm 0.03$} \\
\hline & & 0.25 & & 0.75 & \\
\hline & & 0.28 & & 0.68 & \\
\hline
\end{tabular}




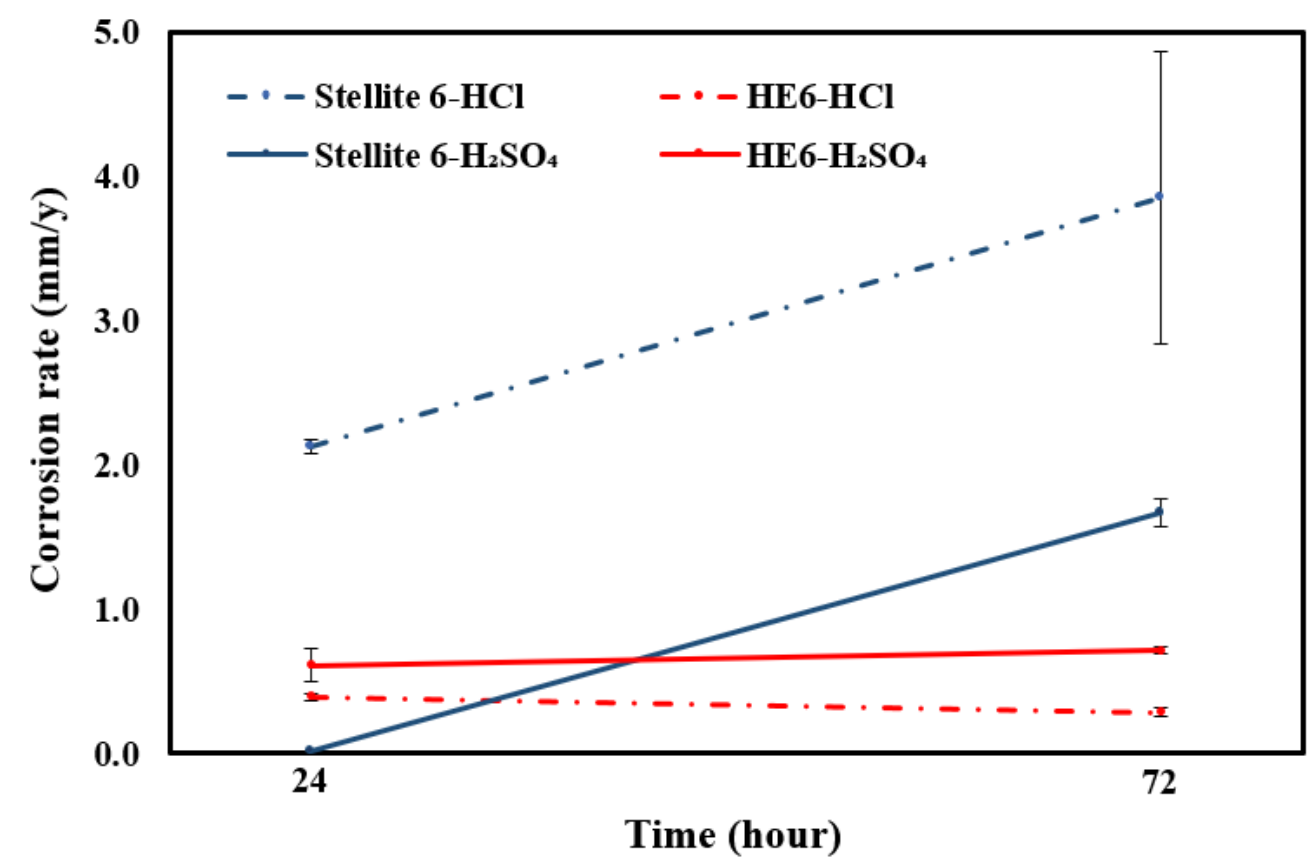

Figure 4-52: Corrosion rate variations with time of the alloys in immersion test.

\subsubsection{Morphology analysis of immersion-tested specimens}

To better understand the corrosion mechanisms of PTA Stellite 6 and PTA HE6 in the immersion tests, the alloy specimens after the immersion tests were examined using SEM/EDX.

For the Stellite 6 specimens soaked in $5 \% \mathrm{HCl}$ solution, the surface morphologies are presented in Figure 4-53, along with the intact surface for comparison. After 24 hour immersion test, the surface morphology of the specimen had been obviously changed according to Figure 4-53 (b). The original microstructure cannot be identified clearly, but the two distinct phases (carbides and solid solution) are generally observed, with the dendrite structure of the solid solution highlighted. Moreover, the surface appears covered with imperfect oxide films. After longer immersion test (72 hours), the specimen surface almost does not show any characteristics of microstructure and is fully covered with corrosion products including oxides debris, as shown in Figure 4-53 (c). Compared with the surface morphology of the specimen tested for 24 hours, 
the oxide films on the specimen surface tested for 72 hours were much damaged during the longer immersion test. Further analysis of the PTA Stellite 6 specimen surface immersion-tested in 5\% $\mathrm{HCl}$ solution for 72 hours was performed on the light and dark regions with EDX. As presented in Figure 4-54 and Figure 4-55, the light region contains higher $\mathrm{O}$ and $\mathrm{Cr}$ contents and lower $\mathrm{Co}$ content, compared with the dark region. This reveals that the light region is corrosion products, mainly $\mathrm{Cr}$ oxide debris, while the dark region should be the solid solution substrate because of high Co and Cr contents, which were exposed due to breaking of the oxide film.

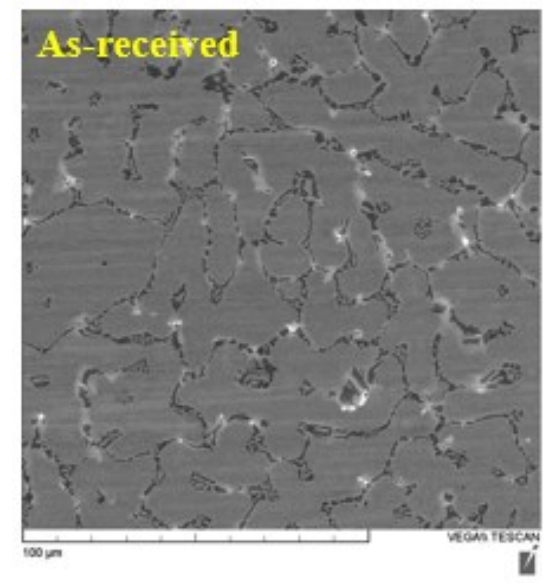

(a)

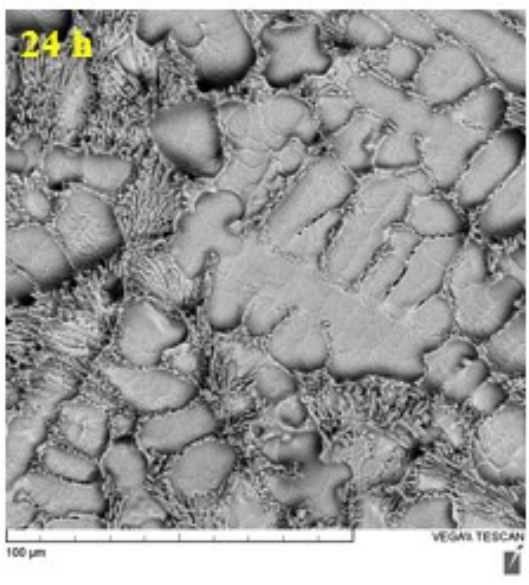

(b)

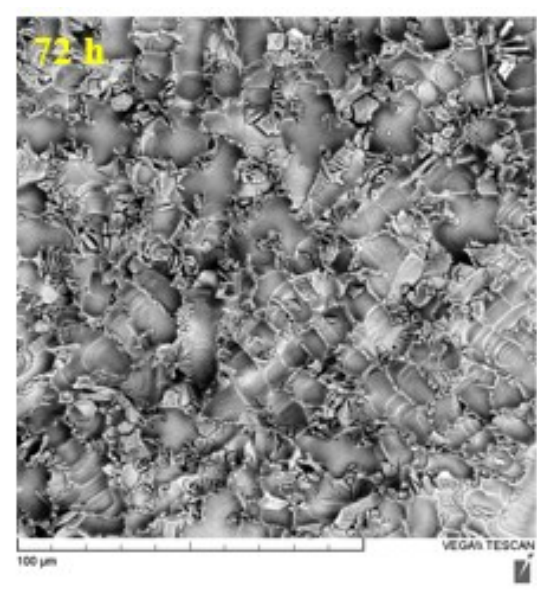

(c)

Figure 4-53: SEM surface morphology of PTA Stellite 6 specimen: (a) as-received, (b) immersion-tested in $5 \% \mathrm{HCl}$ solution for 24 hours, (c) immersion-tested in $5 \% \mathrm{HCl}$ solution for 72 hours. 

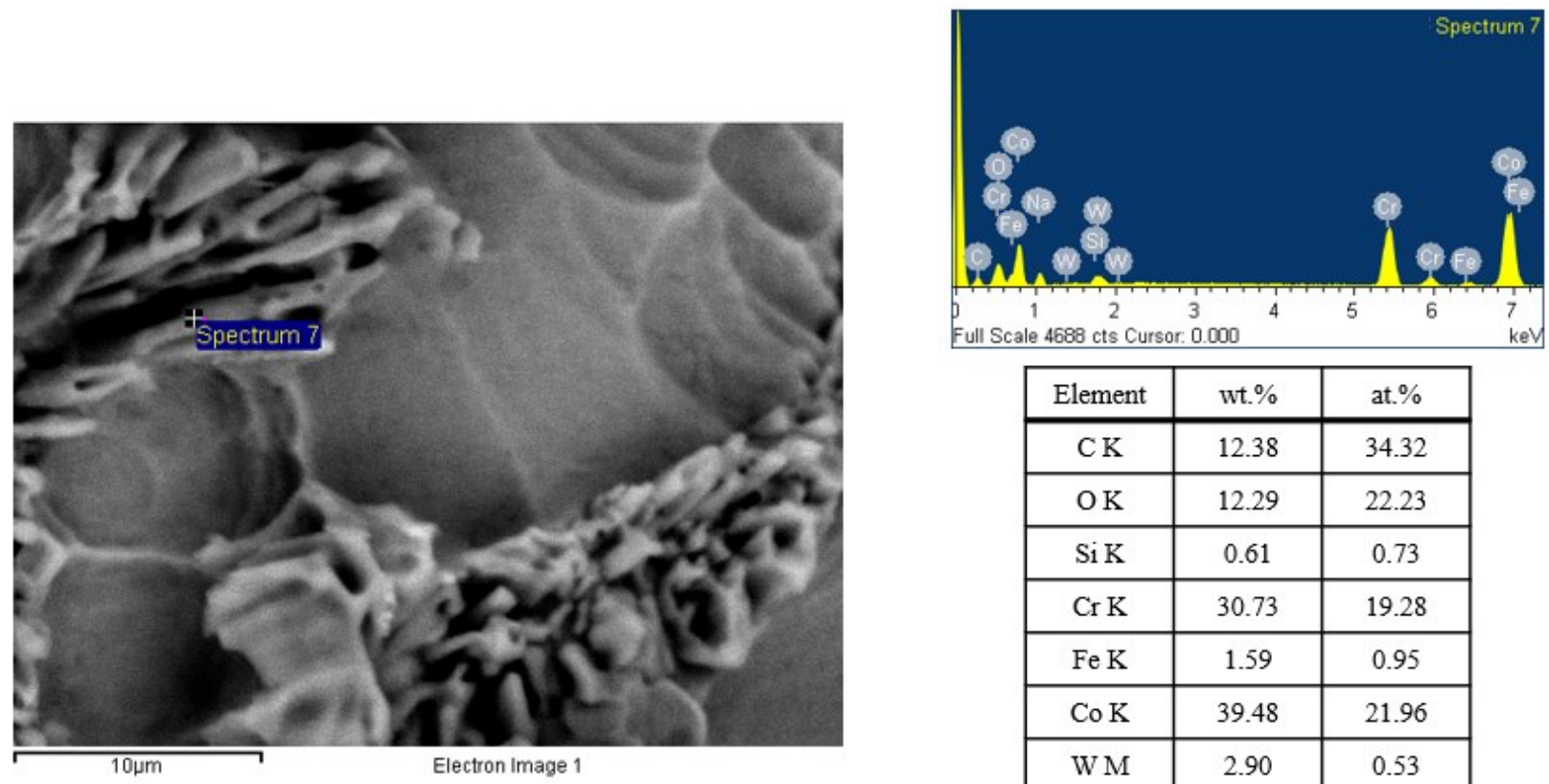

\begin{tabular}{|c|c|c|}
\hline Element & wt.\% & at.\% \\
\hline $\mathrm{C} \mathrm{K}$ & 12.38 & 34.32 \\
\hline $\mathrm{O} \mathrm{K}$ & 12.29 & 22.23 \\
\hline $\mathrm{Si} \mathrm{K}$ & 0.61 & 0.73 \\
\hline $\mathrm{Cr} \mathrm{K}$ & 30.73 & 19.28 \\
\hline $\mathrm{Fe} \mathrm{K}$ & 1.59 & 0.95 \\
\hline $\mathrm{Co} \mathrm{K}$ & 39.48 & 21.96 \\
\hline $\mathrm{W} \mathrm{M}$ & 2.90 & 0.53 \\
\hline & & \\
\hline Totals & 100.00 & \\
\hline
\end{tabular}

Figure 4-54: EDX analyses of the light region in PTA Stellite 6 specimen surface after immersion tested in 5\% $\mathrm{HCl}$ solution for 72 hours.
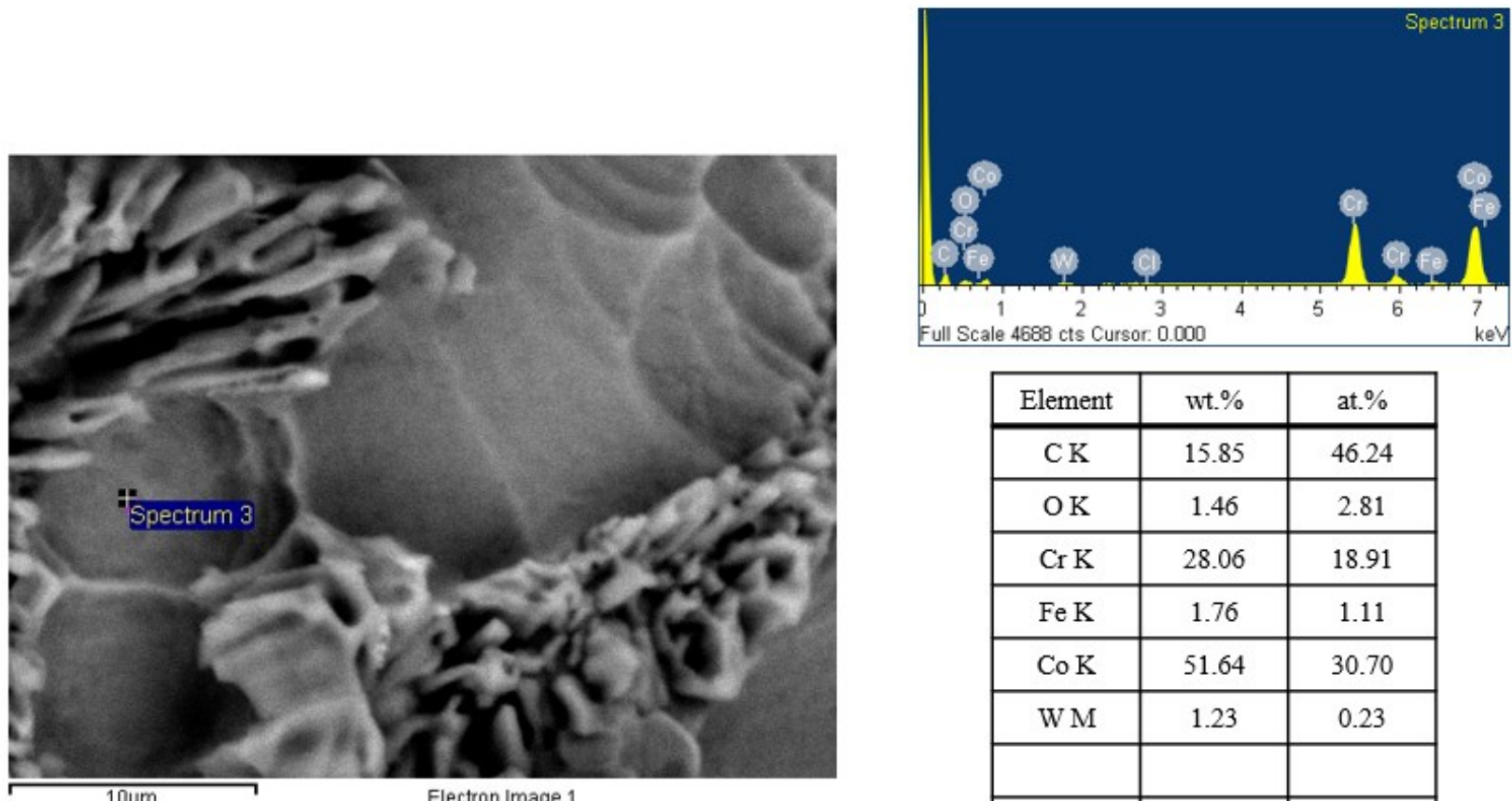

\begin{tabular}{|c|c|c|}
\hline Element & wt.\% & at.\% \\
\hline $\mathrm{C} \mathrm{K}$ & 15.85 & 46.24 \\
\hline $\mathrm{O} \mathrm{K}$ & 1.46 & 2.81 \\
\hline $\mathrm{Cr} \mathrm{K}$ & 28.06 & 18.91 \\
\hline $\mathrm{Fe} \mathrm{K}$ & 1.76 & 1.11 \\
\hline $\mathrm{Co} \mathrm{K}$ & 51.64 & 30.70 \\
\hline $\mathrm{W} \mathrm{M}$ & 1.23 & 0.23 \\
\hline & & \\
\hline Totals & 100.00 & \\
\hline
\end{tabular}

Figure 4-55: EDX analyses of the dark region in PTA Stellite 6 specimen surface after immersion tested in 5\% $\mathrm{HCl}$ solution for 72 hours. 
Furthermore, it is noted that localized corrosion had occurred at the interface area between Cr-rich carbides and Co-based solid solution. This is because there was a potential difference between the Cr-rich carbide phase and Cr-depleted solid solution zone, thus a tiny electrochemical cell could form at the interface where Cr-rich carbide referred to the cathode and the surrounding solid solution performed as the anode in the chemical reaction. Meanwhile, according to the previous studies in immersion corrosion of Cr-containing steels and alloys [101][102], protective $\mathrm{Cr}_{2} \mathrm{O}_{3}$ can form in the solid solution region since free $\mathrm{Cr}$ was liable to lose electrons so as to form $\mathrm{Cr}$ oxide, while $\mathrm{Cr}$-rich carbides may form $\mathrm{Cr}$-rich carbonate. The $\mathrm{Cr}$ oxide is tough and can protect the solid solution from further corrosion, but $\mathrm{Cr}$-rich carbonate is loose and friable which can easily fall off from the surface. However, at the interface between Cr-rich carbides and the solid solution, due to $\mathrm{Cr}$ depletion, the ability of $\mathrm{Cr}$ oxide formation was reduced, thus the interface region was easier corroded, as demonstrated by the surface morphologies shown in Figure 4-53 (b) and Figure 4-53(c).

The SEM surface morphologies of PTA Stellite 6 immersion-tested in $10 \% \mathrm{H}_{2} \mathrm{SO}_{4}$ solution are shown in Figure 4-56. Different from the alloy tested in $\mathrm{HCl}$ solution, the surface did not change much when the specimen was tested in $10 \% \mathrm{H}_{2} \mathrm{SO}_{4}$ solution, even for longer testing duration (72 hours). The microstructure of the alloy can be clearly identified. This indicates that the alloy surface was less damaged in $10 \% \mathrm{H}_{2} \mathrm{SO}_{4}$ solution, resulting in a lower corrosion rate, as reported in Table 4-9. 


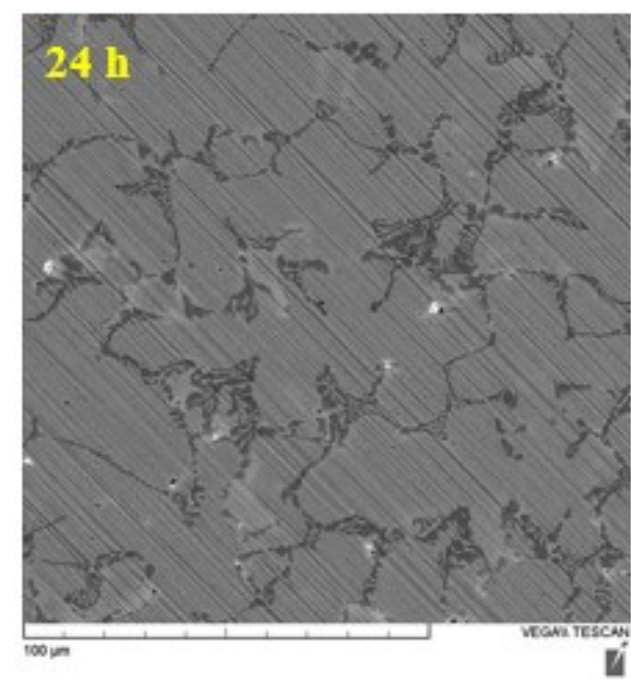

(a)

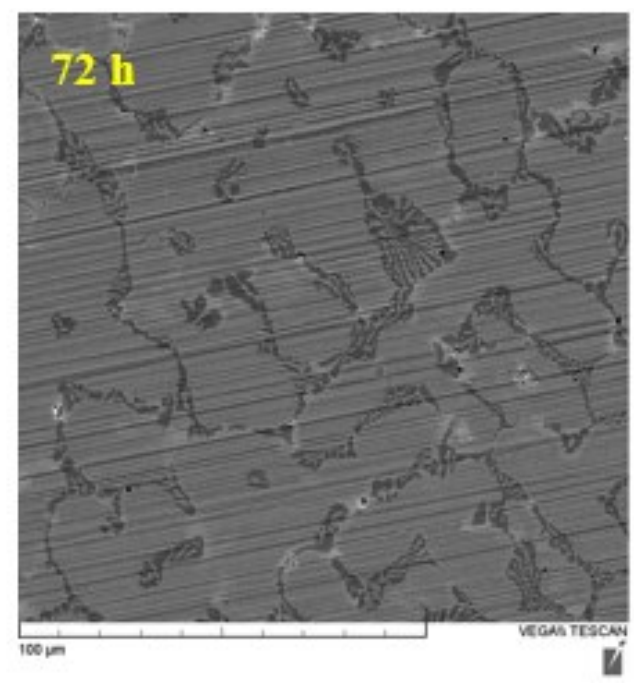

(b)

Figure 4-56: SEM surface morphology of PTA Stellite 6 immersion-tested in $10 \% \mathrm{H}_{2} \mathrm{SO}_{4}$ solution (a) for 24 hours, (b) for 72 hours.

As for PTA HE6 specimens tested in 5\% $\mathrm{HCl}$ solution, a similar inference can be obtained from the surface morphologies shown in Figure 4-57, compared to PTA Stellite 6, but the surface of PTA HE6 was evidently less damaged. After 24 hours of immersion test, the PTA HE6 surface morphology had some changes, but the microstructure can still be identified, as seen in Figure 4-57 (b). The main change in surface morphology is observed in the interface region between the carbide phase and solid solution where obvious corrosion had occurred. The corrosion products include carbonate and oxides. After longer test ( 72 hours), the corrosion was enhanced, as observed in Figure 4-57 (c), the carbides/solid solution interfaces became blunter and the corrosion products were increased. 


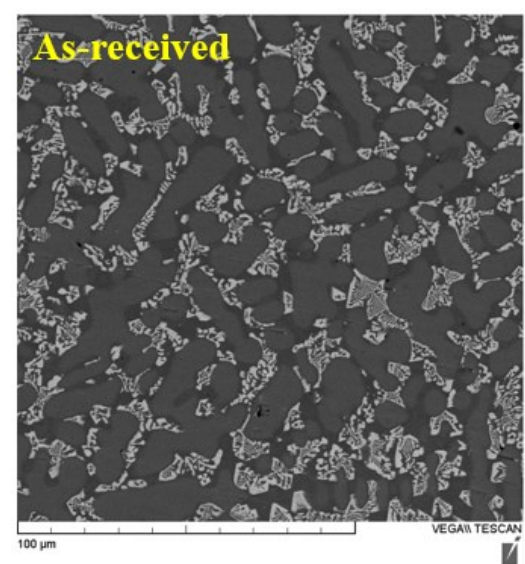

(a)

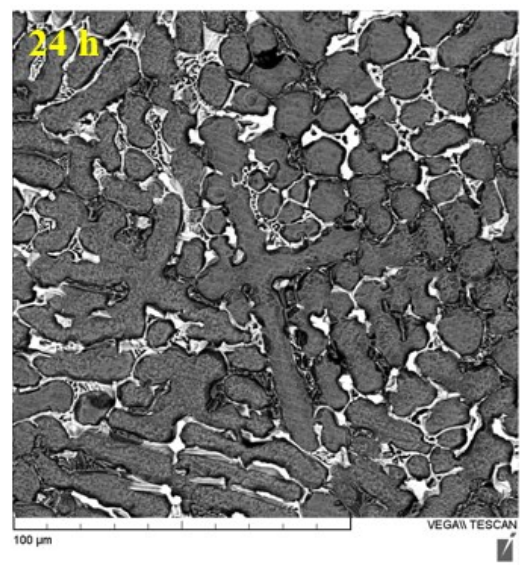

(b)

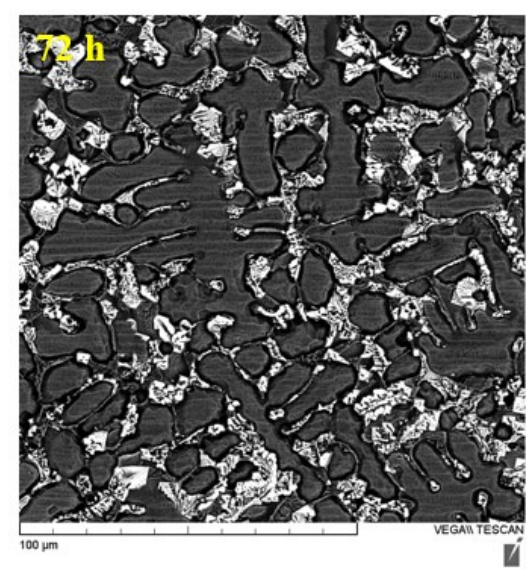

(c)

Figure 4-57: SEM surface morphology of PTA HE6: (a) as-received, (b) immersion-tested in $5 \% \mathrm{HCl}$ solution for 24 hours, (c) immersion-tested in 5\% $\mathrm{HCl}$ solution for 72 hours.

In $10 \% \mathrm{H}_{2} \mathrm{SO}_{4}$ solution, from the surface morphologies shown in Figure 4-58, PTA HE6 is less resistant to corrosion than in $5 \% \mathrm{HCl}$ solution, in particular, for longer testing duration (72 hours), which is consistent with the corrosion rate results reported in Table 4-9. As demonstrated in Figure 4-58 (b), although the microstructure can be somewhat observed, the specimen surface had changed much, with corrosion products covering both the carbides/solid solution interfaces and solution matrix where oxides formed. Furthermore, in addition to the interfaces of different phases, the solution matrix also exhibited localized corrosion — pitting when the PTA HE6 specimen was subjected to a longer immersion test in $10 \% \mathrm{H}_{2} \mathrm{SO}_{4}$ solution. 


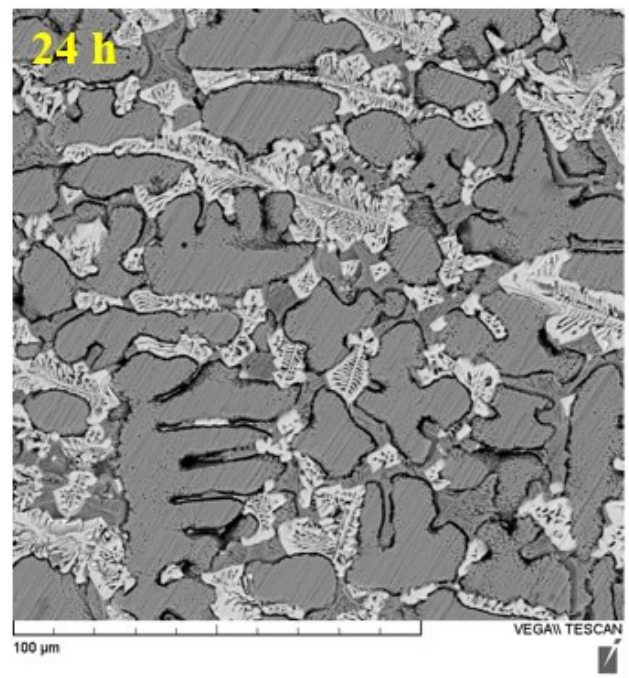

(a)

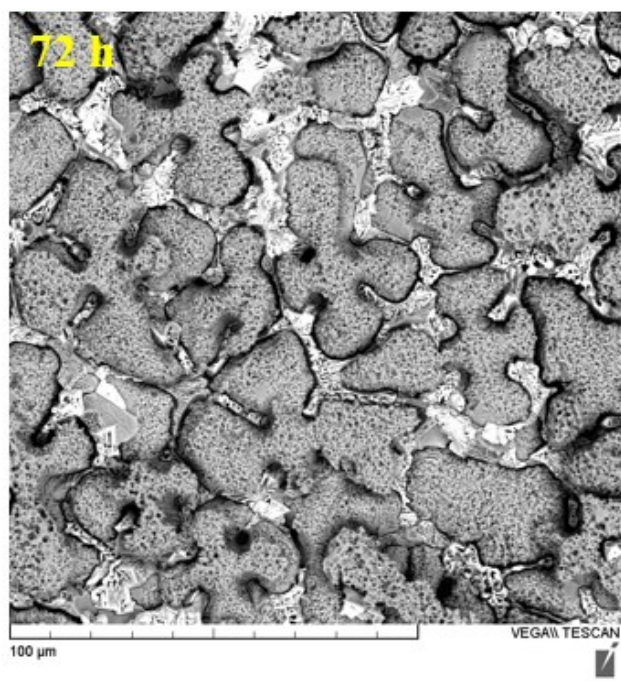

(b)

Figure 4-58: SEM surface morphology of PTA HE6 immersion-tested in $10 \% \mathrm{H}_{2} \mathrm{SO}_{4}$ solution for (a) 24 hours and (b) for 72 hours.

From the surface morphology analyses using SEM, it can be suggested that PTA Stellite 6 and PTA HE6 exhibited similar corrosion mechanisms in both acidic solutions. First, both alloys were able to form oxide films on their surfaces when they were immersed in corrosive environments, but the oxide films were not stable and may be broken with the immersion test proceeding. Second, Stellite 6 and PTA HE6 have similar microstructure; both consist of distinct phases — solid solution and carbides/intermetallics, therefore, localized corrosion preferentially occurred at the interface between the carbides and solid solution matrix. Finally, the solid solution matrices are not perfectly homogenous, thus the potential difference between the constituents in the solid solution did exist, which created electrochemical cells, also leading to pitting corrosion. 


\subsection{Discussion on Experimental Results}

The chemical composition of the novel high entropy alloy, HE6 alloy, is designed based on the HEA concept that the alloy has five or more principal elements with large mixing of entropy $(1.73 R, R$ is gas constant). Thus, HE6 alloy has the equiatomic Co-Cr-Fe-Ni composition with a large amount of $\mathrm{W}$, and a small amount of C (0.96 wt.\%). This special chemical composition offers this alloy a microstructure that possesses the features of both HEAs and Stellite alloys. Similar to Stellite alloys, the microstructure of HE6 consists of various carbides and intermetallics embedded in a solid solution matrix, but unlike Stellite alloys, the FCC solid solution of HE6 is not Co-based, instead, it contains multi-element structures (Co, Cr, Fe and Ni). Compared with the benchmark of Stellite alloy family, Stellite 6, the carbides and intermetallics in HE6 alloy are more diverse, also the overall volume fraction of these strengthening agents is nearly doubled.

Generally, the hardness of a material depends on the volume fraction of strengthening agents precipitated in the material. However, the hardness test results on PTA and SPS specimens of Stellite 6 and HE6 show that Stellite 6 is harder than HE6, in particular, between the SPS specimens. This implies that, first, the solid solution matrix of Stellite 6 is harder than that of HE6 due to high Co concentration with the strengthening of large atom size $\mathrm{W}$; second, $\mathrm{Cr}-\mathrm{rich} \mathrm{M}_{23} \mathrm{C}_{6}$ in Stellite 6 is harder than $\mathrm{Cr} / \mathrm{Fe}$-rich $\mathrm{M}_{16} \mathrm{C}$ in HE6. The SPS HE6 specimen from large-size powder is slightly harder than that from small-size powder. The significant increase in hardness for SPS Stellite 6 specimen should be the result of $\mathrm{M}_{2} \mathrm{C}$ (mainly $\mathrm{W}_{2} \mathrm{C}$ ) precipitation. It is generally accepted that the harder the material, the more resistant to wear it is. Consistent with the hardness test results, the dry-sliding wear tests demonstrated that Stellite 6 was better than HE6 in wear resistance, in about one-order difference. 
The electrochemical tests on PTA HE6 and PTA Stellite 6 specimens in $3.5 \% \mathrm{NaCl}, 2 \%$ $\mathrm{HCl}$ and $10 \% \mathrm{H}_{2} \mathrm{SO}_{4}$ solutions showed that both alloys were able to form protective oxide films (Cr-rich) on the specimen surface with increasing potential owing to high content of $\mathrm{Cr}$ in the alloys so that the corrosion resistance of these alloys strongly depended on the behavior of the oxide films. Since Cr depletion occurred at the carbides/solid solution interfaces, which can result in discontinuity of oxide films on the specimen surface, thus generating localized corrosion (pitting). According to the electrochemical impedance spectroscopy (EIS) test and cyclic polarization test results, Stellite 6 performed better than HE6 in all corrosive media because of larger polarization resistance. This corrosion behavior corresponded to the microstructures of the two alloys. Larger volume fraction of carbides and intermetallics created more interfaces in HE6 thus promoting more sites for electrochemical corrosion reactions. However, immersion tests on PTA HE6 and Stellite 6 specimens revealed that HE6 had a lower corrosion rate in 5\% $\mathrm{HCl}$ and $10 \% \mathrm{H}_{2} \mathrm{SO}_{4}$ solutions for longer test duration, indicating better corrosion resistance in both reducing and oxidizing environments. Also, HE6 maintained a similar level of corrosion rate with the immersion test proceeding, while Stellite 6 exhibited a drastic increase in corrosion rate when the test duration was extended. This implied that the oxide films on HE6 alloy had better repairing ability in long-time corrosion condition. 


\section{Chapter 5: Conclusions and Future Work}

\subsection{Summary of the Work Completed}

A novel cobalt high entropy alloy, designated as HE6, was created in this research, with the aim at reducing Co content in Stellite alloys hence the cost of the alloys, but retaining the similar level of material properties to conventional Stellite alloys, for example, Stellite 6, which is the most popular Stellite alloy and a benchmark in Stellite alloy family, by taking advantages of HEAs. The new HEA has equiatomic Co-Cr-Fe-Ni composition (22 at.\%) with a considerably high content of W, and small amounts of C and Mo, having large mixing of entropy (1.73R).

The HE6 and Stellite 6 alloy specimens were fabricated from the alloy powders via PTA and SPS process, respectively. The raw powders of HE6 and Stellite 6 alloy were examined using SEM for powder morphology and particle size distribution, and investigated under DSC test for phase transformation temperatures, as well as studied with XRD for phase identification. The microstructures of PTA and SPS specimens of HE6 and Stellite 6 were analyzed using $\mathrm{SEM} / \mathrm{EDX} / \mathrm{XRD}$. The phases present in the microstructures were identified and the volume fractions of strengthening agents were estimated using the image processing software, ImageJ.

The overall hardness of PTA and SPS specimens of HE6 and Stellite 6 was determined on both microscale and macroscale. The wear resistance of the specimens was evaluated using a pinon-disc tribometer in dry-sliding mode. The wear loss and friction coefficient for each specimen were determined; the wear mechanisms of PTA and SPS specimens of HE6 and Stellite 6 were studied based on the wear test results, along with the morphology analyses of the worn surfaces using SEM. 
Electrochemical test was used to investigate the corrosion behavior of PTA HE6 and PTA Stellite 6 specimens in $3.5 \% \mathrm{NaCl}, 2 \% \mathrm{HCl}$ and $10 \% \mathrm{H}_{2} \mathrm{SO}_{4}$ solutions; the cyclic polarization curves for the specimens tested in each solution were obtained and hence the corrosion parameters were calculated with the system software.

Immersion test was conducted on PTA HE6 and PTA Stellite 6 specimens in 5\% $\mathrm{HCl}$ and $10 \% \mathrm{H}_{2} \mathrm{SO}_{4}$ solutions to further study the corrosion performance of the alloy specimens. The weight loss due to corrosion was evaluated for each specimen in the solutions and the corroded surfaces of the specimens were analyzed with SEM/EDX to understand the corrosion mechanisms of the alloys.

The experiment results from the performance evaluations of PTA and SPS specimens of HE6 and Stellite 6 were discussed with respect to their microstructures evolved in the PTA and SPS processes. The influence of the manufacturing method on the microstructure developed and hence the final properties of these alloys were delineated. The hardness, wear and corrosion performance of HE6 were assessed in comparison with Stellite 6, hence the significance of developing cobalt HEA was justified. 


\subsection{Conclusions from the Present Research}

From the work completed, reported in previous chapters and summarized above, the following conclusions can be derived from the present research.

1) With the unique chemical composition that takes the features of both Stellite alloys and HEAs, the microstructure of the novel cobalt high entropy alloy, HE6, exhibits the main characteristics of Stellite alloys such that it consists of various carbides and intermetallics distributed in a solid solution matrix.

2) The equiatomic Co-Cr-Fe-Ni composition with the addition of $0.96 \mathrm{wt} . \% \mathrm{C}$ and $19 \mathrm{wt} . \%$ W renders HE6 alloy a complex phase system. Unlike Stellite alloys which have Co-based solid solution (FCC Co or/and HCP Co), the solid solution matrix of HE6 is composed of FCC multi-element structures ( $\mathrm{Co}, \mathrm{Cr}, \mathrm{Fe}$ and $\mathrm{Ni})$. Also, the carbides and intermetallics in HE6 alloy are more diverse than that in Stellite alloy.

3) There is also a difference in carbides between Stellite alloy and HE6 alloy; the main carbide phase of Stellite 6 is Cr-rich, while that of HE6 alloy is $\mathrm{Cr} / \mathrm{Fe}$-rich.

4) Although PTA and SPS material manufacturing methods operate in different mechanisms, the phases formed in the bulk HE6 specimens from these two manufacturing processes are the same, only varying in ratio. However, the two manufacturing processes can result in distinct microstructure morphologies for this alloy. Initial powder size of HE6 alloy had little influence on the microstructure of the bulk specimen prepared via SPS process.

5) HE6 alloy is not as hard as Stellite 6 although the overall volume fraction of strengthening agents (carbides and intermetallics) in the former is larger. Higher hardness of the latter is attributed to strengthened Co-based solid solution matrix and harder Cr-rich carbides, compared to the FCC solid solution with mixed $\mathrm{Co}, \mathrm{Cr}$, Fe and $\mathrm{Ni}$ structures and $\mathrm{Cr} / \mathrm{Fe}-$ rich 
carbides in the former. In consistence with the hardness, HE6 alloy is less resistant to drysliding wear than Stellite 6.

6) Under electrochemical impedance spectroscopy (EIS) and cyclic polarization tests, HE6 alloy displayed passivation ability by forming protective Cr-rich oxide films in $3.5 \% \mathrm{NaCl}$, $2 \% \mathrm{HCl}$ and $10 \% \mathrm{H}_{2} \mathrm{SO}_{4}$ solutions, but localized corrosion (pitting) can occur when the oxide films were broken. Compared with Stellite 6, HE6 alloy has more interfaces between different phases owing to larger amounts of carbides and intermetallics present in the microstructure so that it is less corrosion-resistant because of more sites for electrochemical reaction.

7) However, in immersion test, HE6 alloy exhibited better corrosion resistance than Stellite 6 according to lower corrosion rates in $5 \% \mathrm{HCl}$ and $10 \% \mathrm{H}_{2} \mathrm{SO}_{4}$ solutions, and nearly stable with longer testing duration, which indicated better repairing ability of the oxide films in long-time corrosion condition. 


\subsection{Significant Contributions of the Present Research}

Although Stellite alloys display a variety of exceptional properties, which are attributed to two strengthening mechanisms: strengthened cobalt solid solution matrix and various carbides/intermetallics precipitated in the alloys, they are expensive due to the high cost of dominating Co element. For economic applications, Kennametal Stellite Inc., the world-leading manufacturer of Stellite alloys, has proposed a new idea to create new alloys that possess the features of both Stellite alloys and high entropy alloys, with the aim of reducing the material cost. The cost of the alloys in total contains two parts: the cost of raw materials and the cost of production. A rough and simplified analysis of raw materials can be conducted by calculating the weighted average cost (WAC) for each major element of Stellite 6 and HE6. The weighted average cost can be calculated as

$$
\mathrm{WAC}=\frac{\text { Summation cost of alloying elements }}{\text { Total composition }}
$$

The summation cost equals the unit cost of $\mathrm{Co}, \mathrm{Cr}, \mathrm{W}, \mathrm{Ni}$ and Fe multiplied by their weight percentage. Although Mo and $\mathrm{C}$ also exist in the alloys, their cost impacts are minors since they only occupy $5 \%$ of the weight percentage. According to the market prices of major elements in Stellite alloys and HE6, Table 5-1 summarizes the results of WAC for the most common Stellite alloy and HE6, which shows that HE6 reduces around 40\% cost of Stellite 6. Furthermore, compared to the cost of hot isostatic pressing (HIP), the industrial level SPS facility only costs $10 \%$. In other words, SPS saves more energy due to its shorter thermal cycle. Therefore, HE6 fabricated via SPS results in higher reduction of cost. 
Table 5-1: Weighted average costs of Stellite alloys and HE6.

\begin{tabular}{|c|c|c|c|c|c|c|c|c|c|}
\hline & \multirow{2}{*}{$\begin{array}{l}\text { Unit } \\
\text { price } \\
\text { (U.S. } \\
\text { \$/ton) }\end{array}$} & \multicolumn{2}{|c|}{ Stellite 3} & \multicolumn{2}{|c|}{ Stellite 21} & \multicolumn{2}{|c|}{ Stellite 6} & \multicolumn{2}{|l|}{ HE6 } \\
\hline & & $\begin{array}{c}\text { composition } \\
\text { (wt.\%) }\end{array}$ & $\begin{array}{l}\text { WAC } \\
\text { (U.S. } \\
\text { \$/ton) }\end{array}$ & $\begin{array}{c}\text { composition } \\
\text { (wt.\%) }\end{array}$ & $\begin{array}{l}\text { WAC } \\
\text { (U.S. } \\
\text { \$/ton) }\end{array}$ & $\begin{array}{c}\text { composition } \\
\text { (wt.\%) }\end{array}$ & $\begin{array}{l}\text { WAC } \\
\text { (U.S. } \\
\text { \$/ton) }\end{array}$ & $\begin{array}{c}\text { composition } \\
\text { (wt.\%) }\end{array}$ & $\begin{array}{l}\text { WAC } \\
\text { (U.S. } \\
\text { \$/ton) }\end{array}$ \\
\hline Co & 33000 & 49.8 & \multirow{5}{*}{21826} & 59.5 & \multirow{5}{*}{24721} & 55.3 & \multirow{5}{*}{23322} & 20.1 & \multirow{5}{*}{14280} \\
\hline $\mathrm{Cr}$ & 10000 & 30.5 & & 27 & & 29 & & 18 & \\
\hline W & 10000 & 12.5 & & 0 & & 4.5 & & 19 & \\
\hline $\mathrm{Ni}$ & 16000 & 1.8 & & 2.75 & & 3 & & 20 & \\
\hline $\mathrm{Fe}$ & 1000 & 1.8 & & 3 & & 3 & & 19 & \\
\hline
\end{tabular}

High entropy alloys have been studied extensively and attracted much attention in recent years. They are designed mainly based on solid solution strengthening mechanism, i.e., carbon content in the alloys is kept as low as possible. Different from conventional HEAs, in order to achieve a similar strengthening level to Stellite alloys, the proposed new high entropy alloy, HE6 alloy, contains $0.96 \mathrm{wt} . \% \mathrm{C}$, which promotes the formation of large amounts of carbides.

The present research has completed a preliminary study on HE6 alloy in comparison with the benchmark of the Stellite alloy family, Stellite 6. The experimental results show that this novel alloy has multi-element FCC structure solid solution with diverse carbides and intermetallics. Although the hardness and wear resistance of this alloy are not comparable to Stellite 6, which was expected due to largely reducing Co content, HE6 alloy (HRC $38 \sim 40$ ) is still hard and wear-resistant, compared to most wear-resistant materials. For example, it is harder than Stellite 21 (HRC $28 \sim 32$ ) which is also a popular Stellite alloy for high temperature and corrosion conditions. HE6 alloy exhibited better immersion corrosion resistance than Stellite 6 in both reducing and oxidizing environments for long-time service. The outcomes of this research provided the material industry guidance in developing novel HEAs, leading to the emerging of a new generation of economic materials. 


\subsection{Future Work of the Project}

1) HE6 alloy is the first HEA developed from Stellite alloys. The present research has only provided preliminary outcomes of performance evaluation for this new alloy. Further experimental studies involving erosion, high-temperature oxidation, wear in different modes, corrosion at elevated temperatures, etc., are required before this new material is put into production for industrial applications.

2) HE6 alloy is a Co-Cr-Fe-Ni HEA system with a large amount of $\mathrm{W}$, but as well know, Mo element also plays an important role in Stellite alloys, in particular, providing solid solution strengthening and enhancing corrosion resistance. Therefore, it is worth trying a Co-CrFe-Ni with a large amount of Mo instead of W. Also, element Fe may be replaced by element $\mathrm{Mn}$ in the created HEAs, thus to investigate the difference in material properties when the individual elements are swapped in the alloys.

3) In this research, the bulk HE6 specimens were fabricated via PTA and SPS processes. Although PTA and SPS processes did not make difference in the phase constituents of HE6 alloy, they made distinct microstructure morphologies, which will influence the mechanical properties of this alloy. Therefore, it is necessary to test other manufacturing techniques, for example, laser cladding and 3D printing, to fabricate cobalt HEAs. 


\section{Bibliography}

[1] Kennametal Stellite Inc., "Stellite Alloys [Brochure]," Stellite Alloys at a Glance, 2018. [Online].

Available:https://www.kennametal.com/content/dam/kennametal/kennametal/common/Re sources/Catalogs-Literature/Stellite/B-18-05784_KMT_Stellite_JetKote_Brochure.pdf.

[2] J. R. Davis, Nickel, Cobalt, and Their Alloys. ASM International, 2000.

[3] R. Liu, J. H. Yao, Q. L. Zhang, M. X. Yao, and R. Collier, "Microstructures and Hardness/Wear Performance of High-Carbon Stellite Alloys Containing Molybdenum," Metall. Mater. Trans. A Phys. Metall. Mater. Sci., vol. 46, no. 12, pp. 5504-5513, 2015.

[4] S. Kapoor, R. Liu, X. J. Wu, and M. X. Yao, "Microstructure and Wear Resistance Relations of Stellite Alloys," vol. 4, no. 3, pp. 231-248, 2013.

[5] J.-W. Yeh et al., "Nanostructured High-Entropy Alloys with Multiple Principal Elements: Novel Alloy Design Concepts and Outcomes," Adv. Eng. Mater., vol. 6, no. 5, pp. 299-303, May 2004.

[6] M. C. Gao, P. K. Liaw, J. W. Yeh, and Y. Zhang, High-Entropy Alloys: Fundamentals and Applications. 2016.

[7] W. Steurer, "Single-Phase High-Entropy Alloys - A Critical Update," Mater. Charact., vol. 162, no. January, p. 110179, Apr. 2020.

[8] A. Takeuchi, K. Amiya, T. Wada, K. Yubuta, and W. Zhang, "High-Entropy Alloys with a Hexagonal Close-Packed Structure Designed by Equi-Atomic Alloy Strategy and Binary Phase Diagrams," Jom, vol. 66, no. 10, pp. 1984-1992, 2014.

[9] Y. Zhang, Y. J. Zhou, J. P. Lin, G. L. Chen, and P. K. Liaw, "Solid-Solution Phase Formation Rules for Multi-Component Alloys," Adv. Eng. Mater., vol. 10, no. 6, pp. 534$538,2008$.

[10] S. F. Zhao, G. N. Yang, H. Y. Ding, and K. F. Yao, "A Quinary Ti-Zr-Hf-Be-Cu High Entropy Bulk Metallic Glass with a Critical Size of 12 mm,” Intermetallics, vol. 61, pp. 47$50,2015$.

[11] W. H. Wang, "High-Entropy Metallic Glasses," JOM, vol. 66, no. 10, pp. 2067-2077, Oct. 2014.

[12] O. N. Senkov, J. D. Miller, D. B. Miracle, and C. Woodward, "Accelerated Exploration of Multi-Principal Element Alloys with Solid Solution Phases," Nat. Commun., vol. 6, no. 1, 
p. 6529, May 2015.

[13] Y. Zhang et al., "Microstructures and Properties of High-Entropy Alloys," Prog. Mater. Sci., vol. 61, no. October 2013, pp. 1-93, Apr. 2014.

[14] B. Gludovatz, A. Hohenwarter, D. Catoor, E. H. Chang, E. P. George, and R. O. Ritchie, “A Fracture-Resistant High-Entropy Alloy for Cryogenic Applications," Science (80-. )., vol. 345, no. 6201, pp. 1153-1158, Sep. 2014.

[15] Z. J. Zhang et al., "Nanoscale Origins of The Damage Tolerance of The High-Entropy Alloy CrMnFeCoNi," Nat. Commun., vol. 6, pp. 2-7, 2015.

[16] Y. Zou, H. Ma, and R. Spolenak, "Ultrastrong Ductile and Stable High-Entropy Alloys at Small Scales," Nat. Commun., vol. 6, no. 1, pp. 1-8, Nov. 2015.

[17] P. Koželj et al., "Discovery of a Superconducting High-Entropy Alloy," Phys. Rev. Lett., vol. 113, no. 10, p. 107001, Sep. 2014.

[18] J. Huo et al., "The Magnetocaloric Effect of Gd-Tb-Dy-Al-M (M = Fe, Co and Ni) HighEntropy Bulk Metallic Glasses,” Intermetallics, vol. 58, pp. 31-35, 2015.

[19] Y. Y. Chen, T. Duval, U. D. Hung, J. W. Yeh, and H. C. Shih, "Microstructure and Electrochemical Properties of High Entropy Alloys-a Comparison with Type-304 Stainless Steel," Corros. Sci., vol. 47, no. 9, pp. 2257-2279, 2005.

[20] H. F. Li et al., "In Vitro and in Vivo Studies on Biodegradable CaMgZnSrYb High-Entropy Bulk Metallic Glass,” Acta Biomater., vol. 9, no. 10, pp. 8561-8573, 2013.

[21] P. Huang, R. Liu, X. Wu, and M. X. Yao, "Effects of Molybdenum Content and Heat Treatment on Mechanical and Tribological Properties of a Low-carbon Stellite A Alloy," J. Eng. Mater. Technol. Trans. ASME, vol. 129, no. 4, pp. 523-529, 2007.

[22] C. T. Sims, "A History of Superalloy Metallurgy for Superalloy Metallurgists," in Superalloys 1984 (Fifth International Symposium), 1984, pp. 399-419.

[23] R. Collier, X. Zhang, R. Liu, X. Wu, and M. Yao, "Tribological Performance of Molybdenum Stellite Alloys under Dry-Sliding Wear,” J. Mater. Eng. Perform., vol. 29, no. 2, pp. 1384-1399, 2020.

[24] C. D. Opris, R. Liu, M. X. Yao, and X. J. Wu, "Development of Stellite Alloy Composites with Sintering/HIPing Technique for Wear-Resistant Applications," Mater. Des., vol. 28, no. 2, pp. 581-591, 2007.

[25] M. F. Amateau and W. A. Glaeser, "Survey of Materials for High-Temperature Bearing and 
Sliding Applications," Wear, vol. 7, no. 5, pp. 385-418, Sep. 1964.

[26] W. Betteridge, Cobalt and Its Alloys. Halsted Press, 1982.

[27] A. FRENK and J.-D. WAGNIÈRE, "Laser Cladding with Cobalt-Based Hardfacing Alloys," Le J. Phys. IV, vol. 01, no. C7, pp. C7-65-C7-68, Dec. 1991.

[28] L. E. Falqueto, D. J. Butkus, J. D. B. De Mello, A. C. Bozzi, and C. Scandian, "Sliding Wear of Cobalt-Based Alloys Used in Rolling Seamless Tubes," Wear, vol. 376-377, no. April, pp. 1739-1746, 2017.

[29] L. Gold Standard Corrosion Science Group, "Electrochemical Impedance Spectroscopy (EIS).” [Online]. Available: https://www.gscsg.com/Electrochemical-ImpedanceSpectroscopy.html.

[30] A. Frenk and W. Kurz, "Microstructural Effects on the Sliding Wear Resistance of a CobaltBased Alloy," Wear, vol. 174, no. 1-2, pp. 81-91, May 1994.

[31] I. Radu, D. Y. Li, and R. Llewellyn, "Tribological Behavior of Stellite 21 Modified with Yttrium," Wear, vol. 257, no. 11, pp. 1154-1166, Dec. 2004.

[32] U. Malayoglu and A. Neville, "Comparing the Performance of HIPed and Cast Stellite 6 Alloy in Liquid-Solid Slurries," Wear, vol. 255, no. 1-6, pp. 181-194, Aug. 2003.

[33] M. X. Yao, J. B. C. Wu, and Y. Xie, "Wear, Corrosion and Cracking Resistance of Some W- or Mo-Containing Stellite Hardfacing Alloys," Mater. Sci. Eng. A, vol. 407, no. 1-2, pp. 234-244, Oct. 2005.

[34] B. Cantor, I. T. H. Chang, P. Knight, and A. J. B. Vincent, "Microstructural Development in Equiatomic Multicomponent Alloys," Mater. Sci. Eng. A, vol. 375-377, no. 1-2 SPEC. ISS., pp. 213-218, 2004.

[35] S. Ranganathan, "Alloyed Pleasures: Multimetallic Cocktails," Curr. Sci., vol. 85, no. 10, pp. 1404-1406, 2003.

[36] Gaskell DR, Introduction to the Thermodynamics of Materials, Fifth Edition, 3rd edn. Washington, DC: Taylor \& Francis Ltd, 2008.

[37] R. A. Swalin and S. A. Rice, "Thermodynamics of Solids," Phys. Today, vol. 16, no. 1, pp. 72-74, Jan. 1963.

[38] J.-W. Yeh, "Alloy Design Strategies and Future Trends in High-Entropy Alloys," JOM, vol. 65, no. 12, pp. 1759-1771, Dec. 2013.

[39] A. Takeuchi and A. Inoue, "Classification of Bulk Metallic Glasses by Atomic Size 
Difference, Heat of Mixing and Period of Constituent Elements and Its Application to Characterization of the Main Alloying Element," Mater. Trans., vol. 46, no. 12, pp. 28172829, 2005.

[40] T. B. Massalski, "Comments Concerning Some Features of Phase Diagrams and Phase Transformations," Mater. Trans., vol. 51, no. 4, pp. 583-596, 2010.

[41] U. Mizutani, "Hume-Rothery Rules for Structurally Complex Alloy Phases," MRS Bull., vol. 37, no. 2, pp. 169-169, Feb. 2012.

[42] S. Guo, C. Ng, J. Lu, and C. T. Liu, "Effect of Valence Electron Concentration on Stability of FCC or BCC Phase in High Entropy Alloys," J. Appl. Phys., vol. 109, no. 10, p. 103505, May 2011.

[43] Y. Zhang et al., "Guidelines in Predicting Phase Formation of High-Entropy Alloys," MRS Commun., vol. 4, no. 2, pp. 57-62, 2014.

[44] J. B. Cheng, X. B. Liang, Z. H. Wang, and B. S. Xu, "Formation and Mechanical Properties of Conicufecr High-Entropy Alloys Coatings Prepared by Plasma Transferred Arc Cladding Process,” Plasma Chem. Plasma Process., vol. 33, no. 5, pp. 979-992, 2013.

[45] Z. Shen, M. Johnsson, Z. Zhao, and M. Nygren, "Spark Plasma Sintering of Alumina," J. Am. Ceram. Soc., vol. 85, no. 8, pp. 1921-1927, 2002.

[46] D. C. J. H.B. Feng, Yixiang Zhou, "Principle and Application of Spark Plasma Sintering Technology," Mater. Sci. Technol., vol. 11, no. 3, pp. 327-331, 2003.

[47] Z. Fu, W. Chen, S. Fang, and X. Li, "Effect of Cr Addition on the Alloying Behavior, Microstructure and Mechanical Properties of Twinned CoFeNiA10.5Ti0.5 Alloy," Mater. Sci. Eng. A, vol. 597, pp. 204-211, 2014.

[48] S. Praveen, B. S. Murty, and R. S. Kottada, "Alloying Behavior in Multi-Component AlCoCrCuFe and NiCoCrCuFe High Entropy Alloys," Mater. Sci. Eng. A, vol. 534, no. February, pp. 83-89, 2012.

[49] Tabor D., The Hardness of Metals. New York: Oxford University Press, 1951.

[50] C.-J. Tong et al., "Mechanical Performance of the AlxCoCrCuFeNi High-Entropy Alloy System with Multiprincipal Elements," Metall. Mater. Trans. A, vol. 36, no. 5, pp. 12631271, May 2005.

[51] C.-C. Tung, J.-W. Yeh, T. Shun, S.-K. Chen, Y.-S. Huang, and H.-C. Chen, "On the Elemental Effect of AlCoCrCuFeNi High-Entropy Alloy System,” Mater. Lett., vol. 61, no. 
1, pp. 1-5, Jan. 2007.

[52] A. V. Kuznetsov, D. G. Shaysultanov, N. D. Stepanov, G. A. Salishchev, and O. N. Senkov, "Tensile Properties of an AlCrCuNiFeCo High-Entropy Alloy in As-cast and Wrought Conditions," Mater. Sci. Eng. A, vol. 533, pp. 107-118, Jan. 2012.

[53] L. H. Wen, H. C. Kou, J. S. Li, H. Chang, X. Y. Xue, and L. Zhou, "Effect of Aging Temperature on Microstructure and Properties of AlCoCrCuFeNi High-Entropy Alloy," Intermetallics, vol. 17, no. 4, pp. 266-269, Apr. 2009.

[54] M.-H. Chuang, M.-H. Tsai, W.-R. Wang, S.-J. Lin, and J.-W. Yeh, "Microstructure and Wear Behavior of AlxCo1.5CrFeNi1.5Tiy High-Entropy Alloys," Acta Mater., vol. 59, no. 16, pp. 6308-6317, Sep. 2011.

[55] Z. Tang, L. Huang, W. He, and P. K. Liaw, "Alloying and Processing Effects on the Aqueous Corrosion Behavior of High-Entropy Alloys," Entropy, vol. 16, no. 2, pp. 895911, 2014.

[56] S. Affatato, "Tribological Interactions of Modern Biomaterials used in Total Hip Arthroplasty (THA).," Perspect. Total Hip Arthroplast., pp. 99-116, 2014.

[57] R. Chattopadhyay, Surface Wear Analysis, Treatment, and Prevention. ASM International, 2001.

[58] A. Shrivastava, Introduction to Plastics Engineering. Elsevier, 2018.

[59] J. A. Williams, "Wear and Wear Particles-Some Fundamentals," Tribol. Int., vol. 38, no. 10, pp. 863-870, Oct. 2005.

[60] S. F. and G. C. Maria Cristina Tanzi, Foundations of Biomaterials Engineering. Elsevier, 2019.

[61] J. Paulo Davim, Tribology for Engineers. Woodhead Publishing, 2011.

[62] ASTM G99, "Standard Test Method for Wear Testing with a Pin-on-Disk Standard Test Method for Wear Testing with a Pin-on-Disk Apparatus," ASTM Int., 2017.

[63] Y. Lyu, E. Bergseth, and U. Olofsson, “Open System Tribology and Influence of Weather Condition," Sci. Rep., vol. 6, no. 1, p. 32455, Oct. 2016.

[64] Y. Birol, "High Temperature Sliding Wear Behaviour of Inconel 617 and Stellite 6 Alloys," Wear, vol. 269, no. 9-10, pp. 664-671, 2010.

[65] J. Yao, Y. Ding, R. Liu, Q. Zhang, and L. Wang, "Wear and Corrosion Performance of Laser-Clad Low-Carbon High-Molybdenum Stellite Alloys,” Opt. Laser Technol., vol. 107, 
pp. 32-45, 2018.

[66] P. E. Philip, Fundamentals of Corrosion Mechanisms, Causes, and Preventative Methods. CRC Press, 2010.

[67] X. Z. Zhang, R. Liu, K. Y. Chen, M. X. Yao, and R. Collier, "Electrochemical Study of Corrosion Behavior of Wrought Stellite Alloys in Sodium Chloride and Green Death Solutions," J. Mater. Eng. Perform., vol. 24, no. 9, pp. 3579-3587, Sep. 2015.

[68] H. Piri-Moghadam, M. N. Alam, and J. Pawliszyn, "Review of Geometries and Coating Materials in Solid Phase Microextraction: Opportunities, Limitations, and Future Perspectives," Anal. Chim. Acta, vol. 984, pp. 42-65, Sep. 2017.

[69] O. A. Farghaly, R. S. Abdel Hameed, and A. A. H. Abu-Nawwas, "Analytical Application Using Modern Electrochemical Techniques," Int. J. Electrochem. Sci., pp. 3287-3318, 2014.

[70] C. L. Reedy, R. A. Corbett, and M. Burke, "Electrochemical Tests as Alternatives to Current Methods for Assessing Effects of Exhibition Materials on Metal Artifacts," Stud. Conserv., vol. 43, no. 3, pp. 183-196, Jan. 1998.

[71] J. R. Scully, "Electrochemical Methods for Laboratory Corrosion Testing," in Corrosion Testing and Evaluation: Silver Anniversary Volume, 1990.

[72] D. G. Enos and L. L. Scribner, "The Potentiodynamic Polarization Scan," Solartron Instruments, 2014. [Online]. Available: https://www.ameteksi.com//media/ameteksi/download_links/documentations/library/solartonanalytical/electrochemist ry/technical-report-33-enos-potentiodynamicscan.pdf?dmc $=1 \& l a=e n \&$ revision=eb3fc978-9f69-4406-a544ca7500465e7a\&hash=83B96E0B190FB827E4E48A23F8567B36.

[73] N. Perez, Electrochemistry and Corrosion Science. Boston: Kluwer Academic Publishers, 2004.

[74] ASTM G102, "Standard Practice for from Electrochemical Measurements," ASTM Int., no. Reapproved 2015.

[75] N. Cogger and N. Evans, "An Introduction to Electrochemical Impedance Measurement," Solartron Instruments, 2009. [Online]. Available: https://www.ameteksi.com//media/ameteksi/download_links/documentations/library/solartonanalytical/electrochemist ry/technical-report-06-introduction-to-eis.pdf?dmc=1\&la=en\&revision=6de3 f288-6647- 
46f4-a102-e25a18beac0b\&hash=D48D3BBFA26FE26E28A045AEF2860451.

[76] R. Liu, J. Yao, Q. Zhang, M. X. Yao, and R. Collier, "Effects of Molybdenum Content on the Wear/Erosion and Corrosion Performance of Low-Carbon Stellite Alloys," Mater. Des., vol. 78, pp. 95-106, 2015.

[77] K. Kamal, Y. P. Ding, R. Liu, J. H. Yao, and M. X. Yao, “Corrosion Performance of 700 Series Stellite Alloys in Various Media," J. Mater. Eng. Perform., vol. 28, no. 9, pp. 5605$5615,2019$.

[78] R. Liu, S. Q. Xi, S. Kapoor, and X. J. Wu, "Investigation of Solidification Behavior and Associate Microstructures of Co-Cr-W and Co-Cr-Mo Alloy Systems Using DSC Technique,” J. Mater. Sci., vol. 45, no. 22, pp. 6225-6234, 2010.

[79] M. Kiani Khouzani, A. Bahrami, and M. Yazdan Mehr, "Spark Plasma Sintering of Stellite®-6 Superalloy,” J. Alloys Compd., vol. 782, pp. 461-468, 2019.

[80] F. Kellogg and C. Hofmeister Mock, "The Effect of Current Pathways on Spark Plasma Sintering," in Spark Plasma Sintering of Materials, Cham: Springer International Publishing, 2019, pp. 61-92.

[81] S. Diouf and A. Molinari, "Densification Mechanisms in Spark Plasma Sintering: Effect of Particle Size and Pressure,” Powder Technol., vol. 221, pp. 220-227, 2012.

[82] O. F. Ogunbiyi, T. Jamiru, E. R. Sadiku, O. T. Adesina, L. Beneke, and T. A. Adegbola, “Spark Plasma Sintering of Nickel and Nickel Based Alloys: A Review," Procedia Manuf., vol. 35, pp. 1324-1329, 2019.

[83] H. Yu, R. Ahmed, H. de V. Lovelock, and S. Davies, "Influence of Manufacturing Process and Alloying Element Content on the Tribomechanical Properties of Cobalt-Based Alloys," J. Tribol., vol. 131, no. 1, pp. 1-12, 2009.

[84] R. Lizárraga, F. Pan, L. Bergqvist, E. Holmström, Z. Gercsi, and L. Vitos, "First Principles Theory of the HCP-FCC Phase Transition in Cobalt," Sci. Rep., vol. 7, no. 1, p. 3778, Dec. 2017.

[85] Z. Zhu, C. Ouyang, Y. Qiao, and X. Zhou, "Wear Characteristic of Stellite 6 Alloy Hardfacing Layer by Plasma Arc Surfacing Processes,” Scanning, vol. 2017, pp. 1-8, 2017.

[86] R. Madugundo, N. V. Rama Rao, A. M. Schönhöbel, D. Salazar, and A. A. El-Gendy, "Recent Developments in Nanostructured Permanent Magnet Materials and Their Processing Methods," in Magnetic Nanostructured Materials, Elsevier, 2018, pp. 157-198. 
[87] J.-C. Shin, J.-M. Doh, J.-K. Yoon, D.-Y. Lee, and J.-S. Kim, "Effect of Molybdenum on the Microstructure and Wear Resistance of Cobalt-Base Stellite Hardfacing Alloys," Surf. Coatings Technol., vol. 166, no. 2-3, pp. 117-126, Mar. 2003.

[88] Y. Li, "Electrochemical Study of Corrosion Behavior of Stellite 6 Alloy and 17-4PH Stainless Steel in Amine Solutions,” Carleton University, 2017.

[89] ASTM E384, "Standard Test Method for Microindentation Hardness of Materials," ASTM Int., 2017.

[90] Carleton University, Ed., Operation and Maintenance Instructions: Multi-Purpose Wear Test System (Machine Manual). 2012.

[91] Y. P. Ding, R. Liu, L. Wang, J. H. Li, and J. H. Yao, "Corrosion and Wear Performance of Stellite Alloy Hardfacing Prepared via Laser Cladding," Prot. Met. Phys. Chem. Surfaces, vol. 56, no. 2, pp. 392-404, 2020.

[92] S. Kapoor, "High-Temperature Hardness and Wear Resistance of Stellite Alloys," Carleton University, 2012.

[93] R. Collier, "Experimental and Modeling Study of Sliding Wear Performance for Selected Molybdenum Stellite Alloys," Carleton University, 2019.

[94] ASTM G59, "Standard Test Method for Conducting Potentiodynamic Polarization Resistance Measurements," ASTM Int., no. Reapproved 2014.

[95] ASTM G3, "Standard Practice for Conventions Applicable to Electrochemical Measurements in Corrosion Testing," ASTM Int., no. Reapproved 2019.

[96] ASTM G5, "Standard Reference Test Method for Making Potentiodynamic Anodic Polarization Measurements," ASTM Int., 2014.

[97] H. B. Choe, H. S. Lee, and J. H. Shin, "Experimental Study on the Electrochemical AntiCorrosion Properties of Steel Structures Applying the Arc Thermal Metal Spraying Method," Materials (Basel)., vol. 7, no. 12, pp. 7722-7736, 2014.

[98] F. Rosalbino and G. Scavino, "Corrosion Behaviour Assessment of Cast and HIPed Stellite 6 Alloy in a Chloride-Containing Environment," Electrochim. Acta, vol. 111, pp. 656-662, 2013.

[99] Garmy Instruments, "Basics of Electrochemical Impedance Spectroscopy," 2007. [Online]. Available: https://www.gamry.com/assets/Application-Notes/Basics-of-EIS.pdf.

[100] ASTM G31, "Standard Guide for Laboratory Immersion Corrosion Testing of Metals," 
ASTM Int., 2012.

[101] P. S. Hu, "Improvement in Metal-on-Metal Bearing of Hip Implants with Advanced Stellite Alloys," Carleton University, 2013.

[102] X. Z. Zhang, R. Liu, K. Y. Chen, and M. X. Yao, "Investigation of Corrosion Behavior of Wrought Co-Cr-W Super Alloys," in NACE - International Corrosion Conference Series, 2013. 